\title{
CFD study of hydrodynamic signal perception by fish using the lateral line system
}

\author{
by \\ Mark Andrew Rapo \\ M.S., MIT and WHOI, 2006 \\ A.B., Bowdoin College, 1994
}

Submitted in partial fulfillment of the requirements for the degree of

Doctor of Philosophy

at the

MASSACHUSETTS INSTITUTE OF TECHNOLOGY

and the

WOODS HOLE OCEANOGRAPHIC INSTITUTION

Feb 2009

(c) 2009 Mark Andrew Rapo. All rights reserved.

The author hereby grants to MIT and WHOI permission to reproduce and distribute publicly paper and electronic copies of this thesis document in whole or in part in any medium now known or hereafter created.

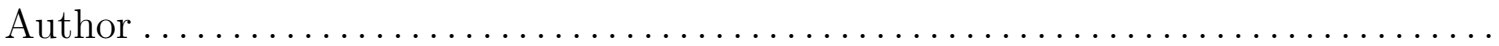

Joint Program in Oceanography/Applied Ocean Science and Engineering Massachusetts Institute of Technology and Woods Hole Oceanographic Institution

Nov 7, 2008

Certified by

Houshuo Jiang

Thesis Supervisor

Associate Scientist, Applied Ocean Science and Engineering

Woods Hole Oceanographic Institution

Accepted by

James C. Preisig Chairman, Joint Committee for Applied Ocean Science and Engineering Woods Hole Oceanographic Institution

Accepted by

David E. Hardt

Chairman of Graduate Studies for the Department of Mechanical Engineering Massachusetts Institute of Technology 


\title{
CFD study of hydrodynamic signal perception by fish using the lateral line system
}

by

\author{
Mark Andrew Rapo
}

\author{
Submitted to the MIT Department of Mechanical Engineering \\ and to the WHOI Department of Applied Ocean Science and Engineering \\ on Nov 7, 2008, in partial fulfillment of the \\ requirements for the degree of \\ Doctor of Philosophy
}

\begin{abstract}
The lateral line system on fish has been found to aid in schooling behavior, courtship communication, active and passive hydrodynamic imaging, and prey detection. The most widely used artificial prey stimulus has been the vibrating sphere, which some fish are able to detect even when the signal velocities to its lateral line are orders of magnitude smaller than background current velocities. It is not clear how the fish are able to extract this signal. This thesis uses a series of computational fluid dynamic (CFD) simulations, matched with recent experiments, to quantify the effects of $3 \mathrm{D}$ fish body parts on the received dipole signals, and to determine signal detection abilities of the lateral line system in background flow conditions.

An approximation is developed for the dipole induced, oscillatory, boundary layer velocity profile over the surface of a fish. An analytic solution is developed for the case when the surface is a wall, and is accurate at points of maximal surface tangential velocity. Results indicate that the flow outside a thin viscous layer remains potential in nature, and that body parts, such as fins, do not significantly affect the received dipole signal in still water conditions. In addition, the canal lateral line system of the sculpin is shown to be over 100 times more sensitive than the superficial lateral line system to high frequency dipole stimuli.

Analytical models were developed for the Mottled Sculpin canal and superficial neuromast motions, in response to hydrodynamic signals. When the background flow was laminar, the neuromast motions induced by the stimulus signal at threshold had a spectral peak larger than spectral peaks resulting from the background flow induced motions. When the turbulence level increased, the resulting induced neuromast motions had dominant low frequency oscillations. For fish using the signal encoding mechanisms of phase-locking or spike rate increasing, signal masking should occur.
\end{abstract}

Thesis Supervisor: Houshuo Jiang

Title: Associate Scientist, Applied Ocean Science and Engineering 


\section{Acknowledgments}

I am very thankful for the funding I received through the Woods Hole Oceanographic Institution Academic Programs Office, and the Coastal Ocean Institute.

I would like to thank Dr. Houshuo Jiang for being my advisor. He is an excellent scientist, and his insights have been invaluable. I have very much enjoyed our exchange of ideas and fruitful discussions. Throughout this process he has treated me like a colleague, and that is a testament to his good character. My experience could not have been better.

I would like to thank Dr. Mark Grosenbaugh, whose encouragement one summer many years ago prompted me to enter this program. He has been a steady guide, and he has helped me navigate through this program at every key juncture. His interests and talents are extensive, and it has been a privelege for me to work with him. I cannot imagine WHOI without him.

I would like to thank Prof. Alex Techet for her selfless devotion to her students. Without a doubt she has enhanced the Joint Program immeasurably. She has been a safe refuge in times of academic stress, and her encouragement to me throughout this process has helped me to succeed. She is a breath of fresh air.

I would like to thank Dr. Tim Stanton for serving as defense chair. It was during one of his classes that I saw not only his dedication to science, but also his concern for the people doing the science. I would like to thank the faculty and scientists of the MIT Department of Ocean Engineering, and of the Applied Ocean Science and Engineering depeartment at WHOI, for inspiring us with your love of science and teaching. Thanks to Prof. Michael Triantafyllou for serving as my academic advisor.

I would like to thank Marsha Gomes, Julia Westwater, Valerie Caron, Leslie Regan, Dan Engelhardt, Ronni Schwartz, and all of the administrative staff of the MIT/WHOI joint program, whose friendly personalities and smiling faces and made them a joy to visit. I would like to thank Jack Zhang, who shared an office next to mine, took care of my plants, and became a friend to me. I would like to thank CIS, and in particular Randy Manchestor, for the much needed help running a linux 
machine. I would like to thank Bob Hendricks for being my hands at Woods Hole when I had computer hardware problems. I would like to thank the custodial and security staff, especially Linda Cataldo, for cheering me up on their rounds when I would be working late into the night.

A special thanks to Prof. Erik Anderson, and his wife Rachel, for being a sounding board during this whole process, and making my wife and I feel sane. They are indeed good friends to us, and we hope that our paths will cross often.

To my long time friends, Min Garabedian, Deryk Eynon, Fred Cobey, and Dan Piper, and to my many new friends, I thank you for your encouragement. Joanna and Dan O'Donnell - you guys helped me rock the defense!! The Paulson's, and the Forget's - thanks for being so supportive to us. To my friend Peter Belengar, and his wife Mingueyame, it is a joy to share this process with you. May our futures be blessed! To all my relatives, I know that you share in my joy. To my new family members, the Kelley's, what fun we have together. I can't imagine this process ending so well without you. To my brother Bradley and his wife Gina, and to my parents, I love you dearly, always. You guys are the best!

To my son Benjamin, your smile greated me every morning, and brightened my day. To my most lovely wife, Shannon. What a blessing that we dated, were married, and had our baby boy during this process. What joy you bring to my life. My step is light, and my heart aflutter in your presence. We did this together.

Finally, to the Lord Jesus Christ, who makes the mountains level and my paths straight, I owe my deepest gratitude and heartfelt love. 


\section{Contents}

1 Introduction $\quad 14$

1.1 Motivation . . . . . . . . . . . . . . . . . . . . . . . . 14

1.2 1st problem - body presence and body parts effects on the received stimulus signal . . . . . . . . . . . . . . . . . 20

1.3 2nd problem - signal extraction in turbulent flow . . . . . . . . . 22

1.4 Thesis Overview . . . . . . . . . . . . . . . . . . . . . 25

2 The effect of the boundary layer and body parts on the received dipole signal in still water: 2D case $\quad 30$

2.1 Introduction . . . . . . . . . . . . . . . . . . . 30

2.2 Methods . . . . . . . . . . . . . . . . . . . . . 33

2.2 .1 General numerical method . . . . . . . . . . . . . . . . 34

2.2 .2 2D dipole next to a wall . . . . . . . . . . . . . 37

2.2 .3 2D dipole next to fish-like bodies . . . . . . . . . . . . 44

2.2.4 Changing dipole locations and orientations study . . . . . 46

2.3 Results . . . . . . . . . . . . . . . . . . . . . . . . . . . . . . . . 49

2.3.1 Oscillating cylinder next to a wall . . . . . . . . . . . 49

2.3.2 Oscillating cylinder next to fish bodies . . . . . . . . . . 55

2.3.3 Changing dipole locations . . . . . . . . . . . . . . . 69

2.4 Discussion . . . . . . . . . . . . . . . . . . . . . . . 75

3 Thresholds of detection and saturation in still water $\quad 80$

3.1 The lateral line system of fish $\ldots \ldots \ldots$. . . . . . . . . 82 
3.1 .1 Canal neuromasts . . . . . . . . . . . . . . . . . . 84

3.1 .2 Superficial neuromasts . . . . . . . . . . . . . . . 98

3.1.3 Models for the Mottled Sculpin . . . . . . . . . . . . 106

3.2 Thresholds of detection and saturation _ . . . . . . . . . . 109

3.2.1 Mechanical definition using neuromast displacement . . . . . . 111

3.2.2 Thresholds of detection and saturation for the Mottled Sculpin in still water enviroments . . . . . . . . . . . . . . . 117

\section{Using CFD to calculate the stimuli to the lateral line system of fish} in still water 124

4.1 Introduction . . . . . . . . . . . . . . . . . . . . . . . . . . . 124

4.2 Methods . . . . . . . . . . . . . . . . . . . . . . 127

4.2.1 Computational domain: 2D strike case set up . . . . . . 128

4.2.2 Computational domain: 3D strike case set up . . . . . . . 129

4.2.3 Computational domain: 3D canonical dipole localization case set up . . . . . . . . . . . . . . . . . 134

4.2.4 Analytical models for the velocity profile and strain rate at a wall due to a dipole source . . . . . . . . . . . . . . . . 134

4.2.5 Numerical determination of the strain rate at a wall due to an oscillating sphere . . . . . . . . . . . . . . . 138

4.2.6 Numerical determination of the pressure gradient at a wall due to an oscillating sphere . . . . . . . . . . . . . . . 139

4.3 Results . . . . . . . . . . . . . . . . . . . . . . . . . 141

4.3.1 Numerical validation for the pressure and pressure gradient signals at a wall due to an oscillating sphere . . . . . . . . . . 141

4.3.2 Numerical validation for the boundary layer velocity profile and strain rate at a wall due to an oscillating sphere . . . . . . . 142

4.3 .3 Sculpin strike cases . . . . . . . . . . . . . . . . . . . . 144

4.3.4 Canonical cases: the signal to the canal lateral line system . . 148

4.3.5 Canonical cases: the signal to the superficial lateral line system 153 
4.3.6 The signal to the lateral line system for a sphere oscillating in the dorsoventral direction . . . . . . . . . . . . 157

4.3.7 Predictions of surface flow on a virtual body using potential flow theory . . . . . . . . . . . . . . . 161

4.3.8 Validation of the strain rate predictions on the fish surface . . 163

4.3.9 Applying results to experiments . . . . . . . . . . . 165

4.4 Discussion . . . . . . . . . . . . . . . . . . . . . . 168

4.4.1 Quantitative determination of canal vs. superficial lateral line sensitivity . . . . . . . . . . . . . . . . . . 169

4.4.2 The effect of body parts on the dipole signal to the lateral line system . . . . . . . . . . . . . . . . . . . 172

4.4.3 Canal and superficial lateral line use in prey detection and localization . . . . . . . . . . . . . . . . 173

5 Thresholds of detection and saturation in moving water $\quad 176$

5.1 Introduction . . . . . . . . . . . . . . . 176

5.1.1 In moving water, saturation for superficial, but not for canal neuromasts . . . . . . . . . . . . . . 177

5.1.2 Background flow and signal masking . . . . . . . . . 178

5.1.3 Biological basis for signal encoding, adaptation, and filtering . 180

5.1.4 Towards a modified definition of threshold of detection and saturation . . . . . . . . . . . . . . . . . . 182

5.2 Methods . . . . . . . . . . . . . . . . . . . . . . . . 184

$5.2 .1 \quad$ Numerical set up . . . . . . . . . . . . . . . . . . . . 184

5.2.2 Thresholds of detection and saturation for the Mottled Sculpin in moving water enviroments . . . . . . . . . . . . 185

5.3 Results . . . . . . . . . . . . . . . . . . . . . 188

5.3.1 Determination of the base-noise level . . . . . . . . . 188

5.3.2 Mean flow characteristics . . . . . . . . . . . . . . . . 190

5.3.3 Neuromast impulse and step responses . . . . . . . . . . . 196 
5.3.4 Determination of the high pass characteristics . . . . . . 206

5.3.5 Laminar flow results . . . . . . . . . . . . . . . . . 208

5.3.6 Turbulent flow results . . . . . . . . . . . . . . . . . 210

5.3.7 Effect of extended fins on signal detection . . . . . . . . . . 230

5.3.8 Threshold of detection in moving water . . . . . . . . . 233

5.4 Discussion ........................... . . 242

6 Conclusion $\quad 250$

6.1 Summary of results . . . . . . . . . . . . . . . . . 250

6.2 Future research . . . . . . . . . . . . . . . . . . . 254

A An oscillating cylinder in still water: THEORY 256 


\section{List of Figures}

1-1 The Mottled Sculpin and prey detection _.......... . 15

1-2 The lateral line system of the Mottled Sculpin . . . . . . . . . 16

1-3 Hair cell system . . . . . . . . . . . . . . . . . . . . . 17

1-4 The canal lateral line system . . . . . . . . . . . . . . . . . . 19

2-1 2D potential flow field: pressure and velocity validation $\ldots \ldots . . .39$

2-2 Oscillating cylinder next to a wall: zoomed in view of grid domain . . 41

2-3 Oscillating cylinder next to fish bodies . . . . . . . . . 45

2-4 Pressure gradient patterns on a wall due to an oscillating cylinder . . 48

2-5 Instantaneous velocity and pressure along the wall due to an oscillating

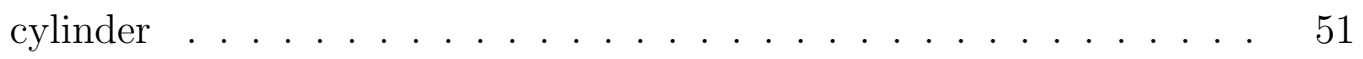

2-6 Velocity field and boundary layer profiles for a cylinder oscillating parallel to a wall . . . . . . . . . . . . . . . . . 5 53

2-7 Velocity field and boundary layer profiles for a cylinder oscillating perpendicular to a wall . . . . . . . . . . . . . . . 54

2-8 Instantaneous velocity and pressure along a NACA 0020 surface from

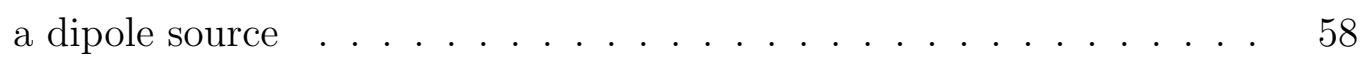

2-9 Instantaneous velocity and pressure along a sculpin surface from a dipole source . . . . . . . . . . . . . . . . . . . . 59

2-10 Instantaneous velocity and pressure along a sculpin surface with fins extended . . . . . . . . . . . . . . . . . 6 60

2-11 Velocity field and boundary layer profiles for a cylinder oscillating parallel to a sculpin . . . . . . . . . . . . . . . . . . . 
2-12 Velocity field and boundary layer profiles for a cylinder oscillating perpendicular to a sculpin . . . . . . . . . . . . . .

2-13 Instantaneous (phase $=\pi / 2$ ) velocity boundary layer profiles on a sculpin surface . . . . . . . . . . . . . . . . .

2-14 Velocity field and boundary layer profiles for a cylinder oscillating parallel to a sculpin with fins extended . . . . . . . . . . . . . . . 65

2-15 Velocity field and boundary layer profiles for a cylinder oscillating perpendicular to a sculpin with fins extended . . . . . . . . . . . 66

2-16 Instantaneous ( phase $=\pi / 2$ ) velocity boundary layer profiles on a sculpin

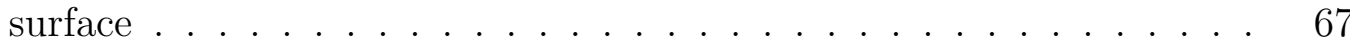

2-17 Instantaneous pressure fields around sculpin bodies near a dipole source 70

2-18 Dipole pressure gradient patterns on a sculpin in close proximity . . . 72

2-19 Dipole pressure gradient patterns on a sculpin a body length away . . 73

2-20 Use of the entire lateral line for source localization . . . . . . . . . . . 74

3-1 Proposed theory of hair-cell polarization (Flock, 1967) . . . . . . . 83

3-2 The lateral line system of the Mottled Sculpin . . . . . . . . . . 85

3-3 Exposed hair bundles in the canal neuromast. . . . . . . . . . . . . 86

3-4 Kalmijn's model of cupular displacement per canal fluid velocity. . . . 88

3-5 Frequency dependent drag coefficient for an oscillating sphere. . . . . 91

3-6 van Netten's model of cupular sensitivity. . . . . . . . . . . . . . . . . 92

3-7 Velocity profile inside a pipe with an oscillating pressure gradient. . . 93

3-8 Velocity profile inside a capillary tube with an oscillating pressure gra-

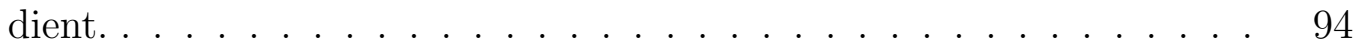

3-9 Filtering properties of a subdermal canal vs. neuromast height. . . . 95

3-10 Filtering properties of a subdermal canal/capillary tube. . . . . . . . 96

3-11 Cupular sensitivity of the canal neuromast system. . . . . . . . . . 97

3-12 Filtering properties of a surface boundary layer vs. neuromast height. 100

3-13 Superficial neuromast model of McHenry and van Netten, 2007. . . . 104

3-14 Cupular sensitivity of the superficial neuromast system. . . . . . . . 105 
3-15 Second order canal system sensitivity for the Mottled Sculpin. . . . . 110

3-16 Detection thresholds for the larval zebrafish. . . . . . . . . . . . 115

3-17 Canal system thresholds for the Mottled Sculpin: 2nd order model . . 119

3-18 Canal system thresholds for the Mottled Sculpin: experimental tuning 122

3-19 Superficial neuromast thresholds for the Mottled Sculpin. . . . . . . . 123

4-1 Computational domain for the 2D cylinder and sculpin . . . . . . . . 130

4-2 Computational domain for the 3D sphere and sculpin . . . . . . . . 133

4-3 Dipole pressure gradient signal on a wall . . . . . . . . . . . . . . 143

4-4 Dipole induced boundary layer velocity profile at the wall . . . . . . 145

4-5 Wall strain rate due to a nearby oscillating sphere . . . . . . . . . 146

4-6 Mottled sculpin strike sequence on a vibrating source:2D . . . . . . 149

4-7 Mottled sculpin strike sequence on a vibrating source:3D . . . . . . 150

4-8 Complete lateral line signal for strike position 3 . . . . . . . . . . . 151

4-9 Dipole signal to the canal lateral line . . . . . . . . . . . . . . . 154

4-10 Dipole signal to the superficial lateral line . . . . . . . . . . . 156

4-11 Sphere oscillating in dorsoventral direction: I . . . . . . . . . . . 158

4-12 Sphere oscillating in dorsoventral direction: II . . . . . . . . . . . . . 159

4-13 Sphere oscillating in dorsoventral direction: III . . . . . . . . . . . . 160

4-14 Surface flow predictions using potential flow theory . . . . . . . . . . 164

4-15 Validation of the strain rate predictions on the fish surface . . . . . 166

4-16 Boundary layer height vs. superficial neuromast height . . . . . . . 175

5-1 Base-noise level: threshold of detection in still water . . . . . . . . . . 189

5-2 Average laminar flow characteristics for sculpin with retracted fins . . 192

5-3 Average laminar flow characteristics for sculpin with extended fins . . 193

5-4 Average turbulent flow characteristics for sculpin with extended fins . 194

5-5 Laminar self flow pressure gradient signals . . . . . . . . . . . . . 197

5-6 Turbulent self flow pressure gradient signals . . . . . . . . . . . . . 198

5-7 Impulse and step responses of the Ruffe canal neuromast . . . . . . . 201

5-8 Impulse and step responses of the Sculpin canal neuromast . . . . . . 202 
5-9 Sinusoidal responses of the Sculpin canal neuromast . . . . . . . . . . 203

5-10 Neuromast model comparison in $8 \mathrm{~cm} / \mathrm{s}$ laminar flow . . . . . . . . . 204

5-11 Hair cell adaptation and filtering effects . . . . . . . . . . . . . 208

$5-122 \mathrm{~cm} / \mathrm{s}$ laminar flow characteristics . . . . . . . . . . . 211

$5-134 \mathrm{~cm} / \mathrm{s}$ laminar flow characteristics . . . . . . . . . . . 212

$5-148 \mathrm{~cm} / \mathrm{s}$ laminar flow characteristics . . . . . . . . . . . 213

5-15 Emergence of prey induced spectral peak in $8 \mathrm{~cm} / \mathrm{s}$ laminar flow . . . 214

$5-162 \mathrm{~cm} / \mathrm{s}$ turbulent flow; rms vel $\sim 0.7 \mathrm{~mm} / \mathrm{s} \ldots \ldots . . \ldots 218$

5-17 Hair cell filtering of the $2 \mathrm{~cm} / \mathrm{s}$ turbulent flow; rms vel $\sim 0.7 \mathrm{~mm} / \mathrm{s} \quad$. 219

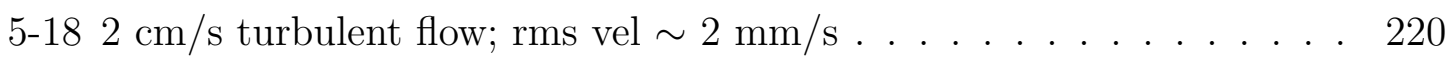

5-19 Hair cell filtering of the $2 \mathrm{~cm} / \mathrm{s}$ turbulent flow; rms vel $\sim 2 \mathrm{~mm} / \mathrm{s}$. . 221

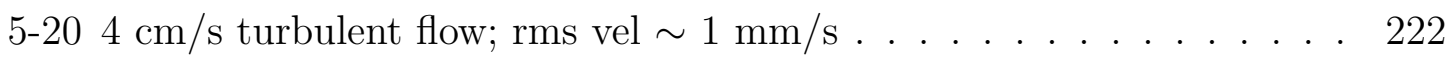

5-21 Hair cell filtering of the $4 \mathrm{~cm} / \mathrm{s}$ turbulent flow; rms vel $\sim 1 \mathrm{~mm} / \mathrm{s}$. . 223

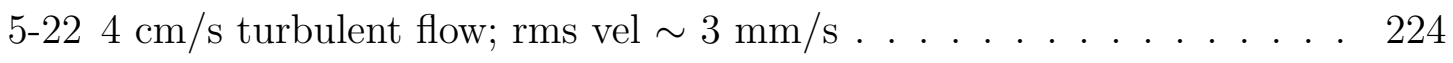

5-23 Hair cell filtering of the $4 \mathrm{~cm} / \mathrm{s}$ turbulent flow; rms vel $\sim 3 \mathrm{~mm} / \mathrm{s}$. . 225

$5-248 \mathrm{~cm} / \mathrm{s}$ turbulent flow; rms vel $\sim 2 \mathrm{~mm} / \mathrm{s} \ldots \ldots . . \ldots 226$

5-25 Hair cell filtering of the $8 \mathrm{~cm} / \mathrm{s}$ turbulent flow; rms vel $\sim 2 \mathrm{~mm} / \mathrm{s}$. . 227

$5-268 \mathrm{~cm} / \mathrm{s}$ turbulent flow; rms vel $\sim 4 \mathrm{~mm} / \mathrm{s} \ldots \ldots . . . . . . .228$

5-27 Hair cell filtering of the $8 \mathrm{~cm} / \mathrm{s}$ turbulent flow; rms vel $\sim 4 \mathrm{~mm} / \mathrm{s}$. . 229

$5-288 \mathrm{~cm} / \mathrm{s}$ turbulent for a sculpin with fins retracted . . . . . . . . . 231

5-29 SNR benefits in the region behind the pectoral fins? . . . . . . . . . 232

5-30 Detection thresholds based on pressure gradient signals . . . . . . . 235

5-31 Detection threshold based on the hair cell response . . . . . . . . . 238

5-32 Signal detection and base-noise level for trout and goldfish . . . . . . 241 


\section{Chapter 1}

\section{Introduction}

\subsection{Motivation}

Nature often shows elegant ways of solving a whole host of engineering problems, and inspiration and understanding of physical principles can be gleaned from studying these solutions. Fish have a lateral line system which enables them to passively sense the environment around them (see figure 1-1). Although general research of the fish lateral line system has been conducted for over 150 years, a more serious pursuit of this study occurred after WWII in hopes of aiding sonar technology (Cahn, 1967).

The lateral line is a mechanosensory system (see figure 1-2) which uses the deflection of hair cells (see figure 1-3) to encode the movements of the adjacent fluid (Bleckmann, 1993). It consists of an organized distribution of canal and superficial (surface) neuromasts, each of which has hair cells covered by a gelatinous covering called a cupula that protrudes into the surrounding fluid. As fluid moves past the neuromast, viscous drag forces cause it to shear, which in turn sends nerve impulses to the brain (see figure 1-4).

In 1966, the first conference devoted solely to the lateral line was held to bring the best researchers together to resolve controversies over the role of the lateral function, and namely, whether it was a pressure or displacement detector (Cahn, 1967). Already the structure and function of the lateral line organ, the neuromast, had been determined (Flock and Wersall, 1962; Flock and Duvall, 1965; Flock, 1967), nerve 

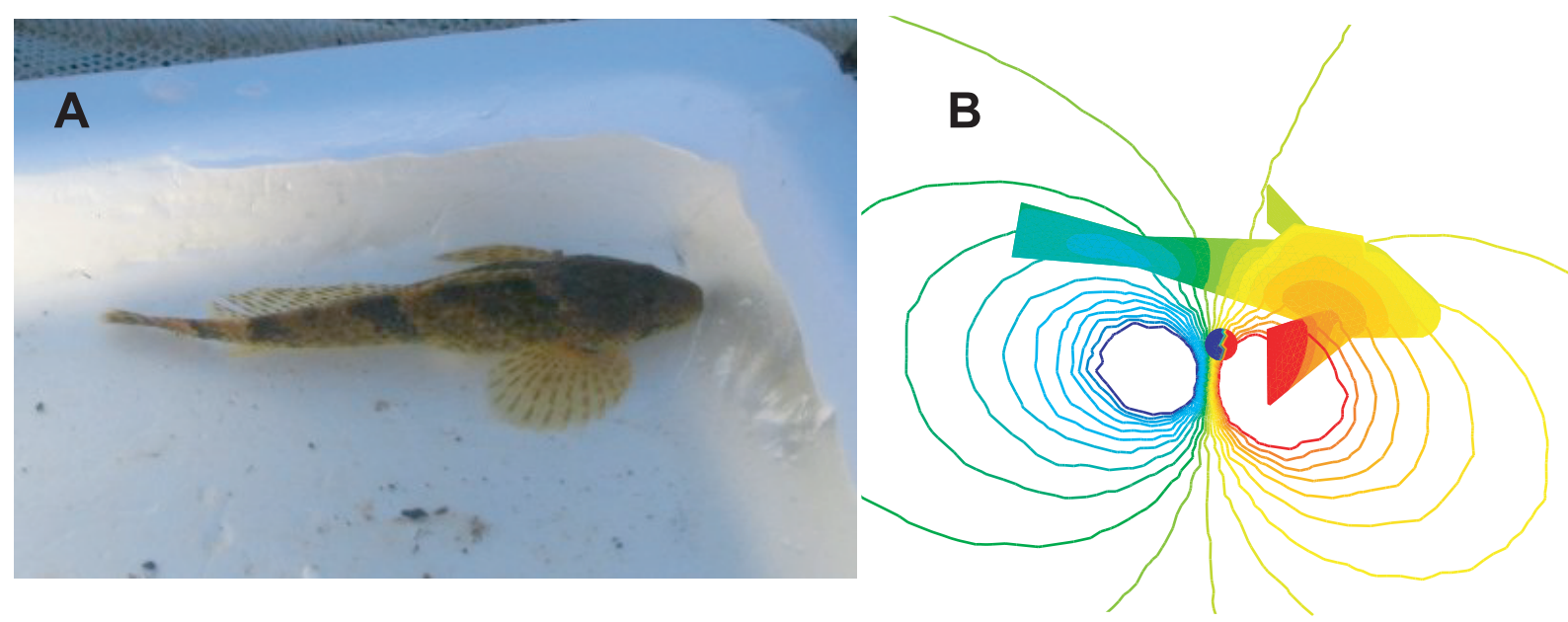

Figure 1-1: (A) The Mottled Sculpin, Cottus Bairdi, is a benthic fish which uses its lateral line system for prey detection. (B) A computer model of the Mottled Sculpin and a nearby artificial prey (blue/red sphere). The vibrating sphere, which represents the flapping of a fish tail, oscillates back and forth, creating a dipole signal that propagates throughout the fluid medium. The interaction of the dipole signal with the fish body is sensed by the lateral line system of the fish. The photograph of the Mottled Sculpin is used with the permission of Mike Guerin.

activity studied (Harris and Flock, 1967; Gorner, 1967), and some evidence found for the role of lateral line in prey detection and localization, active and passive hydrodynamic imaging, surface feeding, rheotaxis (for small currents), schooling, and mating (Dijkgraaf, 1967). These functions of the lateral line have since been confirmed (see Coombs and Montgomery, 1999, for a substantial listing of confirming experiments).

As the demand for the use of autonomous underwater vehicles (AUV's) increases, many functions of the lateral line seem potentially beneficial for the AUV's. If low power / low processing solutions can be found for passive obstacle detection, wake detection, and even schooling with other AUV's, on board resources can be used for other higher order tasks. Fabrication of some prototype mechanical lateral lines have already begun (Yang et al., 2006).

The problem that the fish have solved is being able to extract useful information from the environment around them, even when there is noise from self-flow water motions, or general background flow induced noise. They can detect water displacements from a vibrating source which are magnitudes smaller than the mean flow 


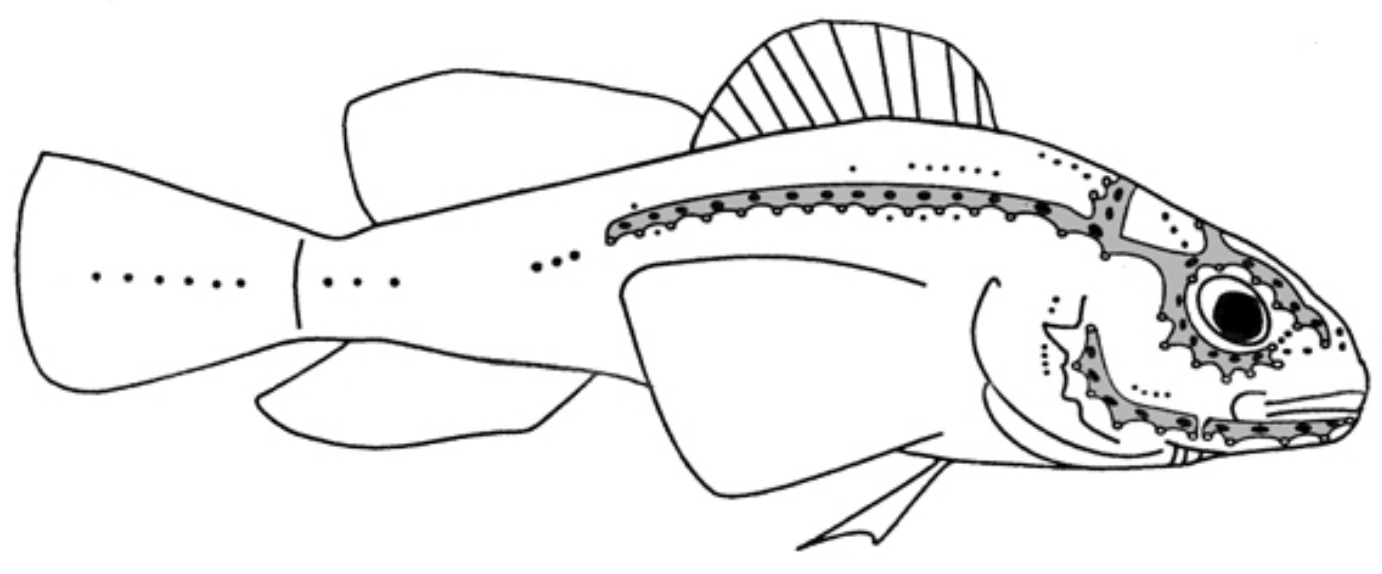

Figure 1-2: The lateral line system of the Mottled Sculpin is represented by the black dots and the solid grey markings. The black dots represent the location of superficial (surface) neuromasts (see figure 1-3). The solid grey markings represent the location of subdermal canals, and the black ovals on the grey markings represnt the canal neuromasts contained within the subdermal canals (see figure 1-4). The drawing is from figure 1C of Yang et al., 2006, and is used with the permission of Sheryl Coombs, and with the permission of the Proceedings of the National Academy of Sciences of the United States of America.

signals present (Kanter and Coombs, 2003; Bassett et al., 2006; Chagnaud et al., $2006)$.

Much of the information that has been learned about the lateral line has been from behavioral and neuro-physiological experiments. Though necessary, it is currently too difficult to measure the hydrodynamic signals present around the fish, the corresponding nerve responses, and the behavior that results. This is the case, if for no other reason, because the act of measuring in one arena affects the outcome in another. For example, obtaining nerve responses requires that the fish be connected with wires, limiting or potentially affecting any natural behavioral responses. Another significant challenge is that the information desired is located in 3D space and time. Selective measuring is required in order to proceed.

Time series of velocity magnitude at particular points in space can be measured using hot-wire anemometry techniques, while velocity time series at individual points in space can be measured using instruments such as an Acoustic Doppler Velocimeter (ADV). Vector velocities projected onto a 2D plane can be measured using Digital 

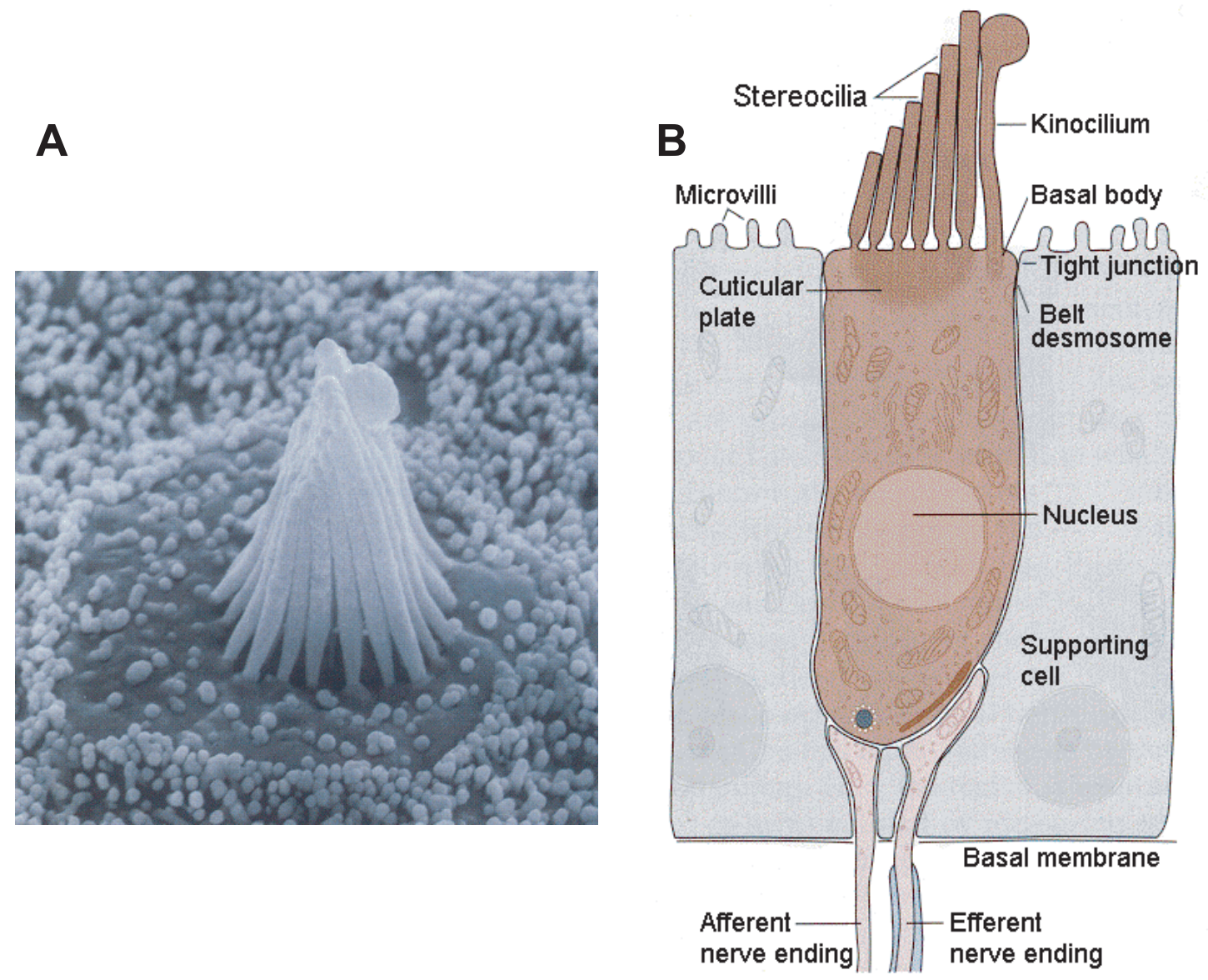

Figure 1-3: (A) A micrograph of the cilia of a hair cell, poking through the surface of the skin. The cilia have a height of $8 \mu \mathrm{m}$ in this picture. (B) A hair cell. The bending of the stereocilia towards the kinocilium causes the hair cell to send nerve impulses to the brain. From Kandel, Schwartz and Jessell, Principles of Neural Science, 4th Edition, 2000, p. 615. Reprinted with the permission of McGraw-Hill. 
Particle Image Velocimetry (DPIV), which tracks the movements of tiny glass beads over successive strobes of a sheet of laser light. Currently, however, there is no way (known to the author) to obtain full velocity information throughout an entire volume without resorting to temporal averaging and situations that are statistically stationary in nature. This limitation on measuring velocity also limits the extraction of instantaneous pressure throughout the water volume.

Numerical simulations using the Navier-Stokes equations, or using Potential flow theory (when simplifications are justified), have been used successfully for hydrodynamic research and analysis. The undulatory motion responsible for tadpole motion (Liu et al., 1997), the self-propelled motion of an anguilliform swimmer (Carling et al., 1998), the flapping fin motion of aquatic flight (Walker and Westneat, 2000), and the vorticity control exercised by swimming fish (Wolfgang et al., 1999; Zhu et al., 2002) are some examples where numerical simulation helped uncover fundamental physical principles. This thesis hopes to use numerical simulation to fill in data gaps not obtained experimentally.

With the basic structure and function of the lateral line fairly well determined, the shift in research focus has been to link realistic environmental situations to corresponding brain stem responses. The goal is to determine what hydrodynamic signals are most relevant to the animals. The flow and signals on the animal will likely be body type and situation specific. It is important to know exactly what hydrodynamic signals are present at the lateral line, and computational fluid dynamics (CFD) can be of significant assistance in quantifying the 3-dimensional, time-dependent hydrodynamic flow fields around the animals.

This thesis will examine two fundamental situations: signals present on the body with no relative water motion (i.e. no noise or still water), and the same signals present on the body with relative water motion (i.e. self-flow noise and/or background flow). It is important to begin with the still water case, so that the received signals can be quantified, and so that any distortions to those signals that occur in flowing water can be empirically determined. 


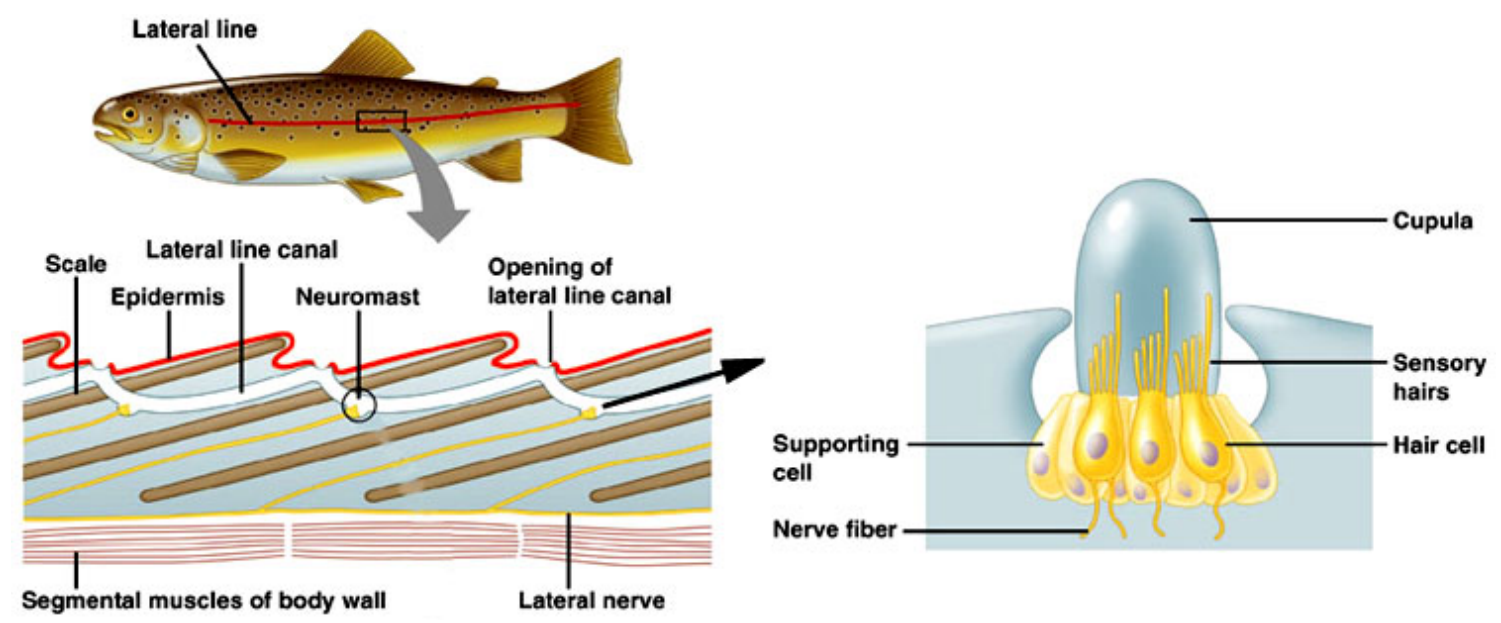

Figure 1-4: The canal lateral line system consists of subdermal canals located beneath the epidermis and scales of a fish. Within the canals are located neuromasts, which are groupings of hair cells. There is generally one neuromast between any two pore openings. The pressure difference between the pore openings drives the fluid within the canal. The cupula is protective, gelatinous covering, which is sheared by the drag force of the passing canal fluid. This in turn causes bending of the cilia, and the hair cells respond by sending impulses to the brain. Copyright and Disclaimer (C- Charles H. Mallery, Department of Biology, University of Miami. All rights reserved. ${ }^{1}$

At the same time, a number of fundamental questions will be answered. How does the presence of the body and its fins affect the received hydrodynamic preygenerated signals to the lateral line? What are the noise characteristics of background flow and self-generated flow to the lateral line? What do hydrodynamic fields of biological stimuli look like to the lateral line in noisy environments, and how can prey localization be pursued by the fish in those situations? Specific experimental cases will be duplicated so that CFD results can be combined with neural and behavioral data.

\footnotetext{
${ }^{1}$ Reference: http://fig.cox.miami.edu/ cmallery/150/neuro/c7.49.12.lateral.line.jpg These web pages are for academic teaching purposes only and contain copyrighted materials from the Biology textbooks used for the courses I teach at the University of Miami. The views and opinions expressed on these course-subject linked-home pages are strictly those of the author, Charles $\mathrm{H}$. Mallery, a faculty member in the College of Arts and Sciences at the University of Miami. These web pages are equivalent to personal home pages and provide an academic class room forum for the self expression of the instructor, Charles H. Mallery. The contents of these pages may include academic subjects, as well as interesting stylistic presentations of class subject material. Although the contents of these linked pages are not in any way an official publication of the University of Miami, we hope that the educational information in them will be useful to students. The contents and the links have not been reviewed or approved by the University of Miami. The University of Miami
} 


\subsection{1st problem - body presence and body parts effects on the received stimulus signal}

The experimentalists alternative to measuring the whole hydrodynamic field has been to use simplified situations which allow for the analytic modeling of the fluid environment, with subsequent verification at select points. From a conceptual point of view, there are two categories of local hydrodynamic signals to model. One is due to external sources, such as nearby obstacles, other schooling fish, or prey. The other is due to self generated flow, from either its own swimming motion or from holding station against a background flow. The reason for the division is that self generated signals must be distinguished by the fish from externally received signals.

Typical external sources could include the changing volume of an air bladder, the flapping of a tail, the wake behind an animal or object, struggling motions, and turbulence. These can be (crudely) represented by monopole, dipole, and multipole sources respectively, although arguably the most biologically relevant signal is the dipole source (Kalmijn, 1988). One reason for this is that higher multi-pole sources decay much more rapidly with distance, so that after short distances, the mono/dipole sources present in the signal dominate. However, in close proximity to the fish, it can be expected that more complex signals are received by the fish lateral line.

The dipole source can be physically realized by oscillating a tiny sphere in water. A typical scenario would have a motor outside of the holding tank, with the sphere inside the tank attached by a thin rod. Having separate structures to hold the motor and to hold the tank isolates any vibrations, and this set up has shown to adequately reproduce a hydrodynamic dipole signal throughout the holding tank when no body is present (Coombs et al., 1996).

accepts no responsibility for the contents of these linked pages. If you think you might be offended by the contents of these pages, you should not continue any further. You may print, reproduce, or use the information and images contained on these biology-course home pages, housed within the University of Miami's Biology Department's server, for non-commercial, personal, or educational purposes only, provided that you (1) do not modify such information, and (2) include both this notice and any copyright notices originally intended and included with such information. 


\section{Analytical solutions - no body present}

The oscillating sphere in an unbounded medium has an analytic solution (Lamb, 1945), and outside a small boundary layer around the sphere surface the hydrodynamic field is effectively potential in nature (van Netten, 2006). Use of a tiny oscillating sphere to represent prey motions began over 40 years ago, and has been the predominant choice ever since (for example, Denton and Gray, 1982; Coombs et al., 1996; Coombs and Conley, 1997a,b; Kanter and Coombs, 2002; Curcic-Blake and van Netten, 2006; van Netten, 2006; Yang et al., 2006). However, these studies which used potential flow theory to arrive at the analytic solution for the pressure field neglected the presence of the body, and the presence of the tank boundaries. The first (and definitive) attempt to include the fish body in the potential flow solution was performed by Hassan $(1985,1992 a, b)$.

\section{Potential flow solutions - axisymmetric body present}

In using potential flow theory, or in using numerical simulations, the fish body is modeled as a solid object. Fish are longitudinally rigid, allowing for relative motions to exist between the fish and the ambient fluid (Denton and Gray, 1982). Although there is certainly sideward motion in swimming motions, this can be neglected for cases of gliding or in sitting on the bottom of a tank. Hassan mathematically treated the fish body like a series of carefully located and calculated point sources and sinks. In 2D cases, Hassan (1985) compared the signals that three fish shapes, circle, oval, and tear drop, would receive when gliding past or approaching directly a cylinder (circle). By allowing the size of the cylinder to increase to almost infinity, the cylinder becomes a wall. In 3D cases, Hassan (1992a,b) used slender body theory to create three axi-symmetric body shapes - prolate spheroid, drop-like, and fish-like. Again this required the careful calculation of the locations and amplitudes of a series of

point sources and sinks. Here the received signal was examined for a fish gliding through water, approaching or gliding along a wall, and being stationary next to an oscillating sphere. 
The first problem: - What effect does the body presence and body parts have on the received dipole signals to the lateral line? Under what conditions is potential flow theory still useful?

What has not been addressed in the literature is how the presence of body parts might affect the local hydrodynamic field and the received signals upon the lateral line. In particular, the pectoral fins can be substantial in size relative to the fish body, and it is important to know how their presence alters the local flow field for the nearby lateral line organs. Modeling body parts, such as thin fins, would make any analytical solutions intractable or unsolvable. This is where the use of computational fluid dynamic simulations becomes necessary, as it is able to handle the added complexity, and produce the complete viscous, space-time results.

\subsection{2nd problem - signal extraction in turbulent flow}

Studies have been conducted which show that the canal lateral line is able to faithfully encode pressure gradient signals from oscillating dipole sources (Coombs et al. 1996). These studies have mostly been done in still water situations, and research is just beginning to examine the effects of water flow stimuli to the lateral line (Voight et al., 2000). Strictly by the mechanics and arrangement of neuromasts, it was predicted that the surface neuromasts would be quickly saturated by the external flows, but that canal neuromasts would remain undisturbed by steady flows. This has proven largely to be true, and some studies suggest that nerve fibers can be classed as either type I or II fibers based on their response or lack of response to steady flows (Englemann et al, 2003; Chagnaud et al., 2006).

\section{The effect of background flow on prey detection}

Experiments were conducted to determine to what extent the surface and canal neuromasts would be able to respond to a dipole source stimulus in background flow 
(Englemann et al. 2002; Bleckmann et al. 2003; Kanter and Coombs 2003; Bassett et al. 2006; Chagnaud et al. 2006) and to a passing object stimulus in background flow (Bleckmann et al. 2003; Englemann et al 2003). From these studies it was concluded that there were two types of neural fibers being innervated. Type I afferent fibers were overstimulated in background flow, and therefore most likely innervated surface neuromasts which respond in some fashion to the velocity of the external flows to which they are exposed. Type II afferent fibers showed little difference in responses to still and running water, and so most likely innervate canal neuromasts, which are only exposed to the external flow through canal pores, and respond to the pressure differences between pore openings.

The characterization that type I fibers are completely overwhelmed by background flow is not quite accurate. There is actually a large variance seen in the masking of type I fibers to an oscillating signal in background flow (Englemann et al. 2002), suggesting that some type I fibers may aid the type II fibers in signal detection even in strong background flows. Recent study of unidirectional flow past a benthic fish shows that there are pockets of recirculation behind the pectoral fins where the flow can be an order of magnitude smaller than the ambient flow (Coombs et al, 2007). This more complex flow around the body may allow for regions where surface neuromasts are not saturated, and can aid in signal detection.

For the lateral line of Gobiomorphus cotidianus, which consists of mostly surface neuromasts, it was able to detect water velocities of approximately $0.1 \mathrm{~mm} / \mathrm{s}$ in still water, but only $1 \mathrm{~mm} / \mathrm{s}$ in water moving at $4.5 \mathrm{~cm} / \mathrm{s}$ (Bassett et al., 2006). For the Mottled Sculpin, the canal neuromasts where able to detect water velocities of 1-10 peak-peak $\mu \mathrm{m} / \mathrm{s}$ in background flows as high as $8 \mathrm{~cm} / \mathrm{s}$, and there was only a two-fold increase in threshold detection for a four-fold increase in water velocity (Kanter and Coombs, 2003). Even when the flow conditions are more noisy (e.g., due to a cylinder placed at various upstream locations of the fish), signal reception characteristics remain similar to those in still and running water backgrounds (Chagnaud et al., 2006). 


\section{Self flow noise (from gliding) - using potential flow theory}

There remains the question of what amplitude and frequency a biologically relevant signal needs to be in order to distinguish itself above the low frequency, high amplitude signal generated from self flow induced noise. One series of mathematical studies determined the steady signal amplitude by using potential flow theory to model the presence of the fish body gliding through the water, gliding past or over obstacles, or receiving signals from an oscillating dipole source (Hassan 1985, 1992a, 1992b, 1993).

Hassan (1985) used potential flow theory to examine the velocity and pressure difference signals received by a $2 \mathrm{D}$ fish lateral line as it glided toward or past a stationary cylinder. Alterations in the received signals caused by the obstacle unambiguously encoded both its size and distance through amplitude and zero-crossing combinations. Unfortunately, the only 2D comparisons for normalized pressure differences come from Hassan comparing one cylinder either passing or directly approaching a stationary cylinder (instead of using fish-like shapes). For a cylinder passing an obstacle (stationary cylinder) in very close range (approaching $1 / 40$ th of the cylinder's width), the induced alterations to the normalized pressure gradient signal had values up to 50, which is much greater than that of self flow generated signals whose values were less than 1 . When the cylinder approached the obstacle (stationary cylinder) head on, changes in current velocity were not noticeable until the front of the moving cylinder was less than one body length away from the obstacle, but then the changes in current velocity increased rapidly with decreasing obstacle distance.

This was followed by 3D studies using potential flow theory to more carefully examine how vector quantities of velocity and pressure gradients could be encoded over the entire surface of the fish (Hassan 1992a, 1992b). When gliding through the water or approaching a plane surface, pressure gradients are strongest in the front of the fish, while almost absent elsewhere (Hassan 1992a). Secondly, altering the body shapes leads to very different stimulus signals. This becomes especially evident when these bodies are gliding above or alongside surfaces, where velocity flow patterns over the surface of each body are unique to each body shape (Hassan 1992b). 
The second problem: What are the noise characteristics of background flow for a streamlined body (retracted fins) versus a bluff body (extended pectoral fins)? Do self-generated flow patterns around the bluff body create pockets of improved signal reception?

Potentially flow theory is not valid inside regions of flow separation, recirculation, or turbulence. Yet the boundary layer of swimming fish has turbulence (Anderson et al., 2001), the boundary layer of stationary fish in background flow has indications of turbulence (Englemann et al., 2002), and benthic fish in background flow has large pockets of recirculation (Coombs et al., 2007). CFD can be used to better quantify what signals the lateral line is receiving in these situations.

\subsection{Thesis Overview}

The main goal of this thesis is to characterize the effects of an oscillating dipole signal to the fish lateral line, taking into account the body-signal interaction, and the presence of background turbulence. Ideally, quantifying when signals will be at the edge of detectability at one extreme, or saturation at the other extreme, will aid our understanding of what the fish might need to do in order to maximize the information content of signals.

This is by nature a 3D problem, and only 3D studies will adequately capture the relevant flow field information. The challenge then is to utilize 2D simulations in such a way as to illuminate any interesting situations that might merit further investigation in the 3D environment of physical experiments or carefully chosen numerical simulations.

One goal of this thesis is to determine how 2D simulations for realistic body shapes compare with 3D simulations. There is a considerable computational cost savings for

running simulations in 2D. For modeling a physically relevant environment needing small scales (mm spacing along the lateral line), and large scales (cm spacing at tank boundaries), there can be an order of magnitude difference in the number of grid cells used between 2D and 3D simulations. This translates into the ability for 
a much larger parameter space to be explored. However, care must be taken to find what aspects of the two-dimensional results will carry forward into the more relevant three-dimensional world.

By choosing to conduct 2D simulations, the local hydrodynamic flow is forced to be in alignment with the direction of the canal system. In a 3D study, the canal system would serve to work in step with surface neuromasts to identify the directionality of the hydrodynamic flow. As the flow moves increasingly to the perpendicular of the canal alignment, stimulation for the canal neuromasts and surface neuromasts in alignment with the canal will die down while stimulation to surface neuromasts aligned to the perpendicular will increase (Hassan, 1993).

The real lateral line will have locations and orientations that specifically sample the pressure and velocity field in a unique way. For example, when the prey is swimming vertically past the fish, in the dorsal/ventral plane, the pressure gradient would be flat around the 2D lateral line (or 3D midplane lateral line), whereas the infraorbital lateral line locations would more readily detect these motions. Hassan (1993) has pointed that the combinations of orientations of the real lateral line sections theoretically allow for unique encoding of the any orientation and location of the dipole source.

Another goal of this thesis is to understand the role of the effect of the surface boundary layer on the received signals. There are in fact a number of different boundary layers that can develop, depending on the local hydrodynamic conditions. Oscillitory flow, such as an oscillating dipole signal created by a flapping tail, will create a boundary layer on the receiving surface that has a height which is inversely proportial to the square root of the oscillation frequency. Depending on the frequencies present, the height of this reduced flow region may be greater than the height of the superficial neuromasts which detect the surface flow. A second type of boundary layer develops from moving background flow, or eqivalently, a gliding motion by the fish. The height of this boundary layer grows larger from the point of attachment at the nose to the back of the fish. It scales according to the Reynolds number. Even within this boundary layer, portions of it may be laminar, and other portions tur- 
bulent. A swimming fish has an undulatory boundary layer (Anderson et al., 2001), whose height and characteristics change with the traveling body waves. Finally, in regions of recirculation, all types boundary layers may be present, including regions of separation and reattachment.

To resolve the boundary layer numerically, there have to be enough samples in space to capture the hydrodynamic changes, and in particular, the gradients. For each type of boundary layer described, there are different spacing resolution requirements. In general, a high frequency oscillatory flow (e.g. $50 \mathrm{~Hz}$ ) has a boundary layer height of about $80 \mu \mathrm{m}$, and demands the most computationally of all boundary layer types. At the writing of this thesis, computational time and memory limits prevent the boundary layer resolution for the 3D, unsteady, background flows.

\section{Thesis structure}

Chapter two will begin the series of 2D simulations. First, the dipole signal next to a wall is simulated, and results are compared to potential flow theory predictions in order to verify the numerical CFD code. In addition, the flow in the boundary layer is compared to an analytical model of oscillatory flow next to a flat plate. Although the conditions under which the analytical model is valid are violated by the nature of flow due to an oscillating dipole signal, it is shown that there are large regions along the wall which are well predicted. A coarse grid model which does not resolve the boundary layer flow, is tested to see how well it predicts the flow just outside the boundary layer. The combination of a coarse grid simulation and the analytical model for flow within the boundary layer, may give results which adequately match the fully resolved boundary layer simulations.

Chapter two will then see how well a coarse grid simulation, in conjunction with the analytical model for flow within the oscillatory boundary layer, predicts results from fully resolved boundary layer simulations around fish-like bodies. In particular, predictions for pressure and velocity around a NACA 0020 shaped body will be compared with those of a mid plane slice through a Mottled Sculpin body with fins

retracted, and with fins extended. Finally, the effect of the pressence of fins on the 
received dipole pressure gradient signal is examined through a parameter study which moves the location and direction of an oscillating dipole signal.

Chapter three will present background material on the biological structure of the lateral line system, and on the analytical models found in the literature which descibe how the neuromasts physically encode local hydrodynamic signals. Models specific to the Mottled Sculpin, a representative benthic fish chosen for study in this thesis, will also be developed. The end goal of this chapter is to have a model which gives a dynamic range of detectable hydrodynamic motions, from the lowest amplitude signals needed for detectability, to the higher amplitude signals which cause saturation and loss of information. There is enough information in the literature to verify this threshold model for cases where there is no background flow.

Chapter four begins the first of the 3D numerical simulations. First, an oscillating sphere next to a wall is simulated, and the pressure and velocity signals are compared with predictions using potential flow theory. A 3D extension of the analytical model for oscillatory flow within the boundary layer next to a wall is checked against numerical simulation results using a resolved boundary layer mesh. The strain rate prediction is validated for points corresponding to maximum velocity along the wall, parallel to the projected axis of vibration, using spheres of various diameter, location, orientation, and oscillation frequency. The relative error in the strain rate prediction over the whole surface is also examined.

Chapter four then begins 3D simulations using a realistic body shape for the Mottled Sculpin. Actual lateral line locations are modeled, so that differences in the effect of the retracted and extended fins can be examined. The first set of simulations match the real approach of a blinded mottled sculpin attacking a vibrating sphere. The sculpin approaches in a step-like fashion, first waiting, then moving, then waiting, and so on (Coombs and Conley, 1997). In each of these situations, the fish changes its body orientation relative to the vibrating sphere, and so the hydrodynamic signals present upon the lateral line will be changing with each step. By setting up identical cases where pectoral fins are extended or retracted, the effect of fins on the received signal can be examined for the first time. 
Chapter four concludes with a parameter study of changing locations and orientations of the dipole signal. The strain rate prediction using a coarse grid model in conjunction with the analytical model for oscillatory flow is validated against a resolved boundary layer simulation for a body with no fins at all. The signals to the canal lateral line system are compared with signals to the superficial lateral line system. The relative diffences in detection threshold levels are compared quantitatively, offering insights into dipole localization.

Chapter five re-examines the threshold of detection and saturation models when background flow is included. At issue is the fact that the flow around a curved body shape creates accelerated flow, causing mean pressure gradients to deflect the canal neuromasts, even to the point of saturation in some cases. The models are utilized to show their response to these strong signals, and methods used by the fish to adapt to these signals are discussed. New definitions for threshold of detection and saturation are presented for use in background flows. The thresholds depend on quantizing oscillatory pressure gradient signals to the lateral line, or alternatively, quantizing the neuromast motions resulting from background flow fluctuations. Chapter five also simulates background flow past the 3D body shapes. The experiment of Kanter and Coombs (2003), with the fish detecting an oscillating dipole signal in background flow, is numerically modeled using Large Eddy Simulation (LES) tools to account for the presence of turbulent structures in the flow. The masking effect of increasing levels of background turbulence on the received dipole signal is quantized, and mechanisms that the fish might use in order to detect the relatively small signal are considered.

A conluding chapter follows, which summarizes the findings of the thesis and looks toward future research. 


\section{Chapter 2}

\section{The effect of the boundary layer and body parts on the received dipole signal in still water: $2 \mathrm{D}$ case}

\section{$2.1 \quad$ Introduction}

The dipole signal has been used extensively in experiments because of its ease of implementation and tractable flow field solutions. The lateral line hair cells are able to detect and encode the dipole signal, as shown by the fact that the firing rate of nerves associated with individual canal neuromasts correspond to the modeled and measured pressure differences upon them (Coombs et al., 1996; Curcic-Blake and van Netten, 2006).

There is a biological basis for an oscillating dipole signal. Denton and Gray (1983) measured the flow field around an artificial tail undergoing flapping motions up to $10 \mathrm{~Hz}$. They used tails $6 \mathrm{~cm}$ long, modeled after herring or sprat tails, and $2.5 \mathrm{~cm}$ in height at the tail end. They compared a flexible tail, a rigid tail, and one which was rigid at the root but flexible at its end, and found that they produced similar flow fields. Quantitatively, at angles perpendicular to the long axis of the tail, the peak-peak pressure declined according to a power function with exponents ranging 
from -1.25 to -2.59 , with -2.1 being the mean, and -2.0 being the expected result for an oscillating sphere. The measured values of pressure followed the expected cosine law for angles greater than about 30 degrees, where 0 degrees is in the direction of the long axis of the tail. The flow field directly behind the tail does not match the flow field of an oscillating sphere since the fish employ thrust dynamics to propel forward, and there is a complex wake left behind.

Comparing absolute values of pressure in the $>30$ degrees region was difficult, since it was hard to know what size sphere to compare the tail to. With the sphere diameter equal to the tail end height, the pressure amplitudes where just over twice those predicted for an oscillating sphere. Denton and Gray have shown that the oscillating sphere has biological grounds for use as a model for swimming fish when looking at the flow field beside the tail of the fish, but not behind it.

Kalmijn (1988) has also argued for the biological importance of a dipole signal. The various motions of a fish can be represented by a combination of monopole sources. When the motion is complex, and potentially irratic, multiple-pole combinations are necessary to represent the flow field produced. However, in the local hydrodynamic flow field (i.e. not the propagating sound wave), the pressure field decays as roughly $(1 / r)^{p}$, where $p$ is the number of poles. Very quickly, in the absence of strong monopole sources (such as an expanding air bladder), the dipole signal $(p=2)$ will dominate the higher-order components of the signal. A gliding fish would produce a dipole signal, while an undulating fish would produce a quadrupole signal $(p=4)$ consisting of the head and tail dipole signals in combination.

In many studies that use a dipole signal to elicit neural or behavioral responses, the presence of the body is not part of the model of the hydrodynamic field. Hassan (1985; 1992a; 1992b) has shown that the presence of the body alters the dipole field, and consequently the pressure and velocity around the fish body is different than what would be calculated or measured at the same location without the body present.

Another consideration is the boundary layer that forms on the surface of the fish. For oscillating signals, this boundary layer is inversely proportional to the square root of the frequency. The frequency dependence of the boundary layer mostly effects 
the velocity around the surface neuromasts, whose heights keep them either fully or partially submerged in the boundary layer at low to high frequencies, respectively. Kalmijn (1988) points out that the neglect of considering the boundary layer has lead to some experimental data being interpreted wrongly.

Though the effect of the body presence has been calculated by Hassan, his work was based on axisymmetric bodies only. Hassan used potential flow theory to obtain the flow field results, which by definition excludes viscous effects, and therefore the boundary layer effects. Windsor (2008) re-examined some of Hassan's scenarios while studying the swimming behavior of the blind Mexican cave fish (Astyanax fasciatus). Windsor compared DPIV results of the hydrodynamic flow field around the fish to that of numerical simulations for a fish gliding through open water, gliding next to a wall, and heading directly at the wall. The 2D and 3D numerical fish bodies were axisymmetric, like in Hassan's case, but the boundary layer was resolved. What has not been considered in the literature is how the presence of fish body parts, namely extended pectoral fins, might alter the dipole signal. Benthic fish such as the Mottled Sculpin use their pectoral fins to hold station against oncoming flows, and hovering fish use their pectoral fins to maintain stability.

The first goal of this chapter is to examine the boundary layer created by an oscillating dipole source next to a surface (wall or fish), and see if there is a way to predict what the flow will look like. It tests the idea that the flow next to a surface is for the most part forced to be parallel to the surface, and locally might appear as a uniformly oscillating fluid above a flat plate. Stokes (1861) provided an analytical solution for a plate oscillating in a still fluid, and the counterpart is that of a fluid oscillating next to a wall. By changing the frame of reference to the plate, whose motion in this analogy would match the wall-parallel motion of the oscillating cylinder, the desired result is achieved. The velocity profile is given as (Lamb, 1945)

$$
U=U_{\infty}\left[e^{-y \sqrt{\frac{\omega}{2 \nu}}} \cos \left(\omega t-y \sqrt{\frac{\omega}{2 \nu}}\right)-\cos (\omega t)\right],
$$

where $U_{\infty}$ is the magnitude of uniform fluid undergoing oscillations, $\omega=2 \pi f$ is the 
radian frequency of oscillation, $y$ is the vertical height above the wall in the boundary layer, $\nu$ is the kinematic viscosity of the fluid, and $t$ is time. The term that is to be predicted is $U_{\infty}$, and potential flow theory may be able to do this.

However, there is a significant difference between a uniform flow oscillating above a plate and that of an oscillating flow above a surface created by a dipole source. In the former case, the pressure gradient is everywhere uniform, and the velocity perpendicular to the surface is everywhere zero. In the latter case, the pressure gradient is not uniform, but has a specific pattern based on the orientation of the axis of oscillation relative to the surface. Secondly, there are stagnation points where the velocity is locally zero, and the velocity perpendicular to the surface is only zero at the surface. Nonetheless, there should be large regions where the flow is well predicted.

The second goal of this chapter is to compare the results of using a fine mesh to resolve the boundary layer around the fish, to that of using a coarser mesh which doesn't resolve the boundary layer, but does effectively predict the flow outside of the boundary layer. This coarse mesh solution, used with the analytical solution described above, may be able to predict the flow within the boundary layer around the fish. Using a coarser mesh can have large savings in computational memory and time when simulating time evolving flows, or any kind of 3D flow.

The final goal of this chapter is to use a series of $2 \mathrm{D}$ simulations to look at what effect extended pectoral fins have on the received dipole signal. The signal that forms under still water conditions will be considered the base signal, which can then be compared to the received dipole signal under moving water conditions.

\subsection{Methods}

The chapter is organized into a series of $2 \mathrm{D}$ numerical simulations. The first simulation models the dipole signal interaction with a wall. It is assumed that the side of the fish will act like a wall to a source in close proximity. The next set of simulations will test the validity of this assumption, and compare how the curvature and finite size 
of a streamlined fish alter the flow field. Then the fish body with extended pectoral fins is included in the simulations. Finally, a parameter study is conducted altering the location and orientation of the dipole field, to see how the signals are altered.

\subsubsection{General numerical method}

There are many numerical schemes available for solving differential equations like the Navier-Stokes equations. The Navier-Stokes equations are a complete description of viscous fluid flow. Solving a particular fluid flow problem is a bit of an art. Computationally, it is impossible to represent all scales of space and time, and the most difficult fluid flow problems involve multiple scales. For instance, when turbulence exists, anisotropic swirling eddies of fully developed flows take the size of the physical boundaries. The energy cascade is to ever smaller motions at ever increasing frequencies, until finally the energy dissipates through motions that have become locally isotropic.

The art of numerical simulations is to make decisions about what to model and what to approximate. Perhaps the most difficult choice is how to grid the domain, or equivalently, discretize the equations. The domain needs to be resolved in the right locations, so that computational time is not wasted in regions where there is not much change in the flow field. In unsteady simulations, the region of grid resolution may need to change as the flow itself evolves. The goal is to reach a grid resolution, such that further increases in the resolution do not change the flow results. This is not always possible, and in those cases, the main aspects of the flow need to be well enough modeled.

In this thesis, the professional grid software GAMBIT $^{\mathrm{TM}}$ is used. $\mathrm{GAMBIT}^{\mathrm{TM}}$ offers the ability to grid both 2D and 3D domains, and offers many choices on how the domain will be meshed. By far the most often chosen method in this thesis is to create

and resolve the boundaries, and then to allow $\mathrm{GAMBIT}^{\mathrm{TM}}$ to mesh the interior of the domain. Since shapes can be irregular, triangular (unstructured) meshes offer an exceptional solution. However, near-wall meshing often does better with more regular meshing, such as quadrilateral structures. 
The solver chosen is the professional software FLUENT ${ }^{\mathrm{TM}}$, which uses the finitevolume method to solve the Navier-Stokes equations for each cell. The equations are spatially averaged over control volumes, whose individual sizes are established during the meshing process. These new equations are discretized, and then linearized in order to create a system of equations which includes unkown variables from its own cell and from neighboring cells. For each unknown variable, the entire domain is solved simulataneously using a Gauss-Seidal iteration scheme along with an algebraic multigrid method. After the momentum equations are solved for the $u, v$, and $w$ velocities, a pressure-correction equation is solved to adjust the pressure and velocity fields so that continuity is satisfied. The pressure is solved in a staggered control-volume configuration (Patankar, 1980), using the PRESTO! (Pressure staggering option) scheme. The process is then iterated until there is convergence to a desired degree of accuracy.

The implicitly linearized equations are solved using the quadratic upwind interpolation (QUICK!) scheme (Leonard, 1991), which defaults to a second-order upwind scheme whenever the grid has non-quadrilateral cells (Barth and Jespersen, 1989). To reduce the number of iterations required for the unsteady, incompressible flows, the PISO (Pressure-Implicit with Splitting of Operators) pressure-velocity coupling scheme is used (Issa, 1986; Ferzieger and Peric, 1996). A deforming mesh model (Demirdzic and Peric, 1990) allows for movement of spatial grid points to adjust for motions of the cylinder (or sphere). Gradients are computed using a Green-Gauss theorem, and temporal discretization uses a first order implicit scheme. Details specific to each simulation are given as needed.

\section{Grid spacing and viscous vs. potential effects}

When inertial forces dominate over viscous forces, that region of the flow can be considered inviscid. However, next to any surface, there will always be a boundary layer where the flow in the inertia dominated region transitions to match the flow attached to the surface. In this region, viscous forces either match or exceed the inertial forces. However, this region can be very small, being proportional to the 
inverse square root of frequency for oscillatory flows, or being dependent on the distance from an initial point of contact on the surface for steady background flows. Schlichting (1979) gives an approximate boundary layer thickness, $\delta$, on the surface in an oscillatory flow as

$$
\delta=\sqrt{2 \nu / \omega},
$$

where $\omega=2 \pi f$ is the angular frequency, and $\nu$ is the kinematic viscosity of the fluid.

How the boundary layer next to a wall or surface is meshed can have a big impact on computation efforts. To accurately model the boundary layer, mesh spacing may need to be on the order of microns. For 2D or 3D geometries, a few layers of micron spaced points can quickly add hundreds of thousands to millions of discretized cells to the computations. For unsteady flows, this can quickly become crippling.

When the grid size next to a surface is larger than the boundary layer, the effects of the boundary layer are diminished or negligible. Under these circumstances, the flow essentially behaves as if it were inviscid in that region. This can be of benefit or deficit, depending on the desired results. For example, when all that is desired is a potential dipole field, a course enough resolution around the oscillating sphere/cylinder will cause the boundary layer effects on the velocity and pressure components to not be modeled. However, to see the effects of an oscillating flow on the velocity profile next to the surface of a wall (or fish body), the grid needs to be resolved.

In the case of a pressure distribution next to the wall or surface of the fish, the boundary layer should present negligible effects, since the pressure passes through almost unchanged. Coarse grid meshing should produce results almost identical to resolved boundary layer meshes. This is valuable for the 3D background flow scenarios, where the canal neuromasts naturally have a coarse $(2 \mathrm{~mm})$ spacing relative to the oscillating boundary layer. Since it is thought that the superficial neuromasts are already saturated by the flow, all that needs to be modeled is the signal to the canal neuromasts. This provides a large computational savings. 


\subsubsection{D dipole next to a wall}

A model case to consider is that of a dipole signal next to a wall. In $2 \mathrm{D}$, this will be an oscillating cylinder next to a wall. The numerical process is carried out in two steps. First, an oscillating cylinder in an unbounded medium (or finite medium where the effects of the boundaries are negligible) is simulated and validated against potential flow theory. The purpose of this is to find the proper grid mesh needed to correctly produce the dipole signal. Secondly, the presence of the wall is introduced. Again, a course mesh near the wall should produce results that can be predicted by potential flow theory, whereas the resolved mesh near the wall will allow for the boundary layer velocity profile to be determined.

\section{Potential flow solution for an oscillating cylinder in an unbounded medium}

For potential flow theory, the potential function for an oscillating cylinder is given as (Lamb, 1931)

$$
\phi=-\left(\frac{a^{2}}{r}\right) U_{0} \cos (\theta) \cos (\omega t),
$$

where $a$ is the radius of the cylinder, $r$ is the radial distance from the center of the cylinder, $\theta$ is the angle from the axis of oscillation, $U_{0}$ is the velocity amplitude, $\omega$ is the angular frequency of oscillation, and $t$ is the time. The radial and tangential velocities can be found by taking their respective gradients of the potential function,

$$
V_{r}=\frac{\partial \phi}{\partial r}=\left(\frac{a}{r}\right)^{2} U_{0} \cos (\theta) \cos (\omega t),
$$

and

$$
V_{\theta}=\frac{1}{r} \frac{\partial \phi}{\partial \theta}=\left(\frac{a}{r}\right)^{2} U_{0} \sin (\theta) \cos (\omega t) .
$$

The pressure is given by the Bernoulli equation for potential flow,

$$
p=-\rho\left[\frac{\partial \phi}{\partial t}+\frac{1}{2}|\nabla \phi|^{2}+g z\right]+F(t) .
$$


When the gradient squared term is small in comparison to the time changing terms (and with gravity ignored), it reduces to

$$
p \approx-\rho \frac{\partial \phi}{\partial t}=\rho\left(\frac{a^{2}}{r}\right) U_{0} \omega \cos (\theta) \sin (\omega t) .
$$

\section{Numerical set up}

To model potential flow around an oscillating cylinder, the correct node spacing around its circumference has to be determined. One issue is the cell centroid location for the cells which lie on the perimeter of the cylinder. Cell centroids should not reside too close the cylinder, which would have the effect of resolving the oscillating boundary layer that develops over the cylinder surface. On the other hand, the cell centroids can not be located too far from the surface, or the desired inviscid flow will also be left unresolved. When meshing with triangular cells, the cell centroid is located $1 / 3$ the distance along the perpendicular bisector of the base leg. Triangular cells whose base lies on the cylinder perimeter leave a gap which can be filled by an inverted triangle. For these cells, whose triangle apex just touches the perimeter of the cylinder and whose base connects the apexes of adjacent triangles, the cell centroid is $2 / 3$ the distance from the apex to the base along its perpendicular bisector. In terms of node spacing, or equivalently the base length of an equilateral triangle, $b$, the cell centroids lie in the range $(\sqrt{5} / 6-2 \sqrt{5} / 6) b$, the average being a little more than $0.5 b$. When the triangles are not equilateral, the location of the centroid can deviate from

this analysis. In these simulations, the lower bound to this range was found to be about $0.3 b$, rather than the $0.37 b$ predicted.

Another issue is the node spacing to surface curvature ratio, $b / r$, which can equivalently be written as $2 \pi / n$, where $n$ is the number of nodes. If this ratio is too large, the cylinder shape may not be accurately modeled, and as the cylinder moves the flow will be mis-modeled. Finally, grid spacing must always be chosen to resolve pressure and velocity gradients, and to capture the temporal aspects of the flow (such as advection). The courant number for the mesh around the cylinder surface is $U_{0} \Delta t / b$, and should be close to or less than unity. $U_{0}$ is the velocity amplitude of the cylinder, 
and $\Delta t$ is the time step.

In a series of grid refinement tests, it was found that having nodes spaced about $1 / 3 \mathrm{~mm}$ apart $(b / \delta \approx 4)$ around the circumference of a cylinder with a $3 \mathrm{~mm}$ radius very accurately reproduced the drag on a cylinder oscillating at $50 \mathrm{~Hz}$ according to potential flow theory (not shown). However, nodes spaced only $1 \mathrm{~mm}$ apart $(b / \delta \approx$ 12.5) was all that was needed to correctly reproduce the pressure and velocity flow field for the same $50 \mathrm{~Hz}$ frequency (figure 2-1). The node spacing to curvature ratio was about 0.1 in the former case, and about 0.3 in the latter.
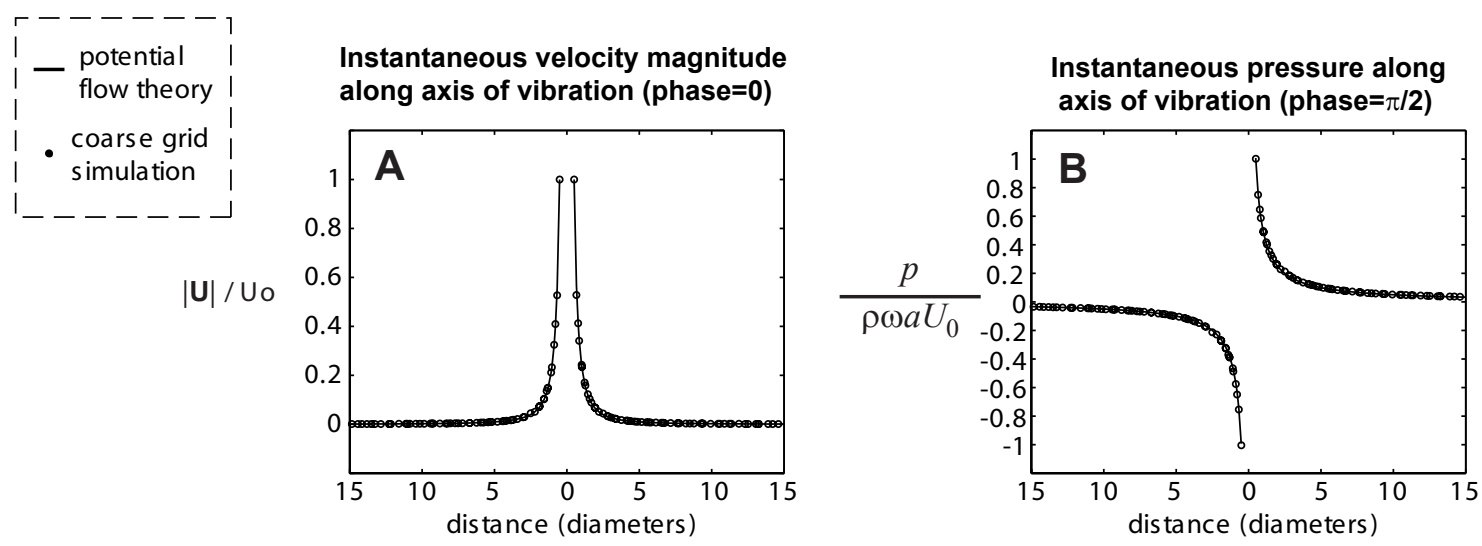

Figure 2-1: 2D potential flow field: pressure and velocity field validation. The node spacing is approximately $1 \mathrm{~mm}$ around the cylinder circumference, and the frequency of oscillation is $50 \mathrm{~Hz}$. This spacing is considered 'coarse', in the sense that it is unable to accurately resolve the boundary layer around the oscillating cylinder. However, this is the desired effect, as it is able to reproduce the 'potential' flow field.

Potential flow equations (eqns. 2.4 - 2.7) show that the velocity and pressure fields scale geometrically according to some function of the ratio of the radius, $a$, to the location in space, $r$. Therefore, the figures are presented in normalized units. The numerical solver, FLUENT ${ }^{\mathrm{TM}}$, runs in dimensional units, and so that is how this information is presented here in the text. A cylinder with a $3 \mathrm{~mm}$ radius was placed at distance of $2.1 \mathrm{~cm}$ from the wall, and it was enclosed by a circular region with a 
$15 \mathrm{~mm}$ radius. In this deformable region, node points were allowed to move as the cylinder oscillated. The outer dimensions were $30 \times 16.3 \mathrm{~cm}$, the bottom $3 \mathrm{~mm}$ next to the wall being assigned to the boundary layer region.

For the coarse grid mesh, the wall boundary layer was meshed with triangular cells whose nodes were spaced $2 \mathrm{~mm}$ apart $(b / \delta \approx 25)$. This coarse spacing appears to be adequate to capture the potential flow field effects near the wall (see figure 2-5), and is probably aided by the fact that the node spacing to curvature ratio is zero. The sides of the designated wall boundary layer mesh were $3 \mathrm{~mm}$ in height, and had a node place at the midpoint, $1.5 \mathrm{~mm}$. For the resolved boundary layer mesh, quadrilateral cells were used. The first cell had a height of $5.5 \mu \mathrm{m}(\delta / 14.55)$, and each of the subsequent 15 cells grew at a rate of 1.4 times the cell beneath it. The base of the quadrilateral cells were all $1 \mathrm{~mm}$ length $(12.5 \delta)$.

The side boundaries above the designated $3 \mathrm{~mm}$ boundary layer space had node lengths beginning at approximately $2.1 \mathrm{~mm}$, and growing at a rate of 1.05 times the previous spacing. The top boundary, far away from the wall and oscillating cylinder, had node lengths $1 \mathrm{~cm}$ apart. The perimeters of the cylinder and of the deforming region had node lengths of approximately $1 \mathrm{~mm}$ apart. GAMBIT ${ }^{\mathrm{TM}}$ filled the entire rest of the space (excluding the boundary layer) with triangular cells. Figure 2-2 shows a zoomed in view around the cylinder and wall for the coarse and resolved boundary layer grids.

The wall boundary was given no-flux and no-slip boundary conditions, corresponding to velocity components being zero. One side boundary was given zero pressure, and the other two sides were assigned symmetry boundary conditions. The cylinder velocity was set to $U_{0} \cos (\omega t)$ along the axis of vibration, with $U_{0}=7 \mathrm{~mm} / \mathrm{s}$, and the frequency of oscillation ( $\omega=2 \pi f$ ) set to $f=50 \mathrm{~Hz}$. By the no slip boundary condition, the fluid velocity at the cylinder surface is equal to the cylinder velocity. The amplitude of the cylinder motion is defined as $U_{0} / \omega$, which means the maximum displacement is about $22 \mu \mathrm{m}$. Therefore, the node displacement within the deforming zone was minimal, since nodes were spaced between 1-2 mm apart within this region. FLUENT $^{\text {TM }}$ solved the unsteady simulations with a time step of $f / 100$, where $f$ is 


\section{Zoomed in view of 2D Grid domain for an oscillating cylinder next to a wall}

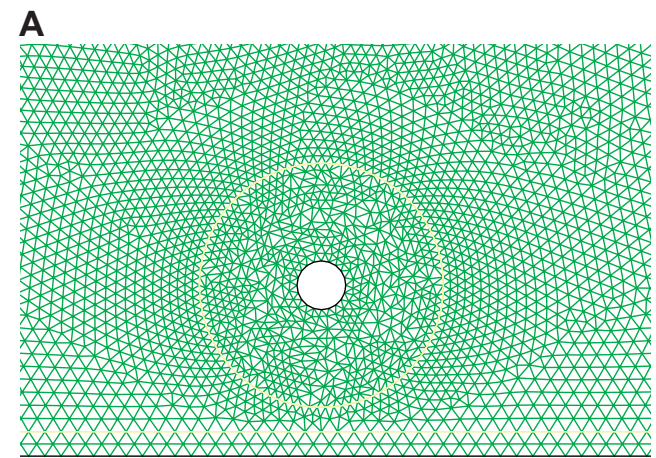

unresolved boundary layer

for "potential flow solution"
B

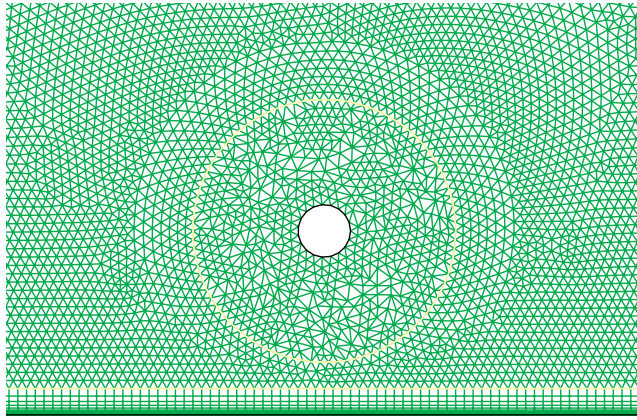

resolved boundary layer

for "viscous flow solution"

Figure 2-2: The grid domain for an unresolved boundary layer ('coarse' grid) and resolved boundary layer. The coarse grid provides the 'potential flow' solution, since it has nodes in the inviscid region, whereas the resolved boundary layer grid has nodes in the viscous layer next to the wall. The cylinders have their centers located at a distance of 7 radii from the wall.

the frequency of oscillation.

\section{Potential flow solution for an oscillating cylinder next to a wall}

Potential flow theory cannot be used to predict the wall-parallel component of velocity in the boundary layer of an oscillating fluid next to a wall, but may be able to predict what can be considered the velocity outside the boundary layer, $U_{\infty}$. Secondly, the wall-vertical component within the boundary layer may be able to be partially predicted by potential flow theory. Finally, since the pressure is not really affected by the boundary layer, potential flow theory should be able to predict the pressure at the wall at all phases of the flow oscillation.

For predicting the vertical velocity component, the solution for a uniform flow oscillating next to a wall does not have a vertical velocity component, and may superpose itself onto the potential flow component. The reason for stating that it will only be partially predicted is because technically the flow next to the wall due to an oscillating cylinder is not uniform, and actually does have a vertical component which varies in magnitude based on the location in the flow field and the phase of the 
oscillation. When the vertical component due to the oscillatory flow is minimal, the potential flow solution for the vertical component in the boundary layer next to the wall should be well predicted.

When potential flow theory is valid (i.e. incompressible, inviscid, irrotational), then $\nabla^{2} \phi=0$ is valid throughout the medium. This is a linear equation, allowing for any $\phi=\sum_{\mathrm{i}} \mathrm{b}_{\mathrm{i}} \phi_{\mathrm{i}}$, as long as the no-flux kinematic boundary conditions are satisfied, $\partial \phi / \partial n=U_{n}$. Here, $\mathrm{b}_{\mathrm{i}}$ are constants and $n$ specifies the normal direction. The method of images may be employed to represent an oscillating cylinder near a wall by superposing a second, mirrored oscillating cylinder an equidistant across the imaginary line that would be the wall. This is better illustrated using Cartesian coordinates, so that for a cylinder with axis of oscillation parallel to the x-axis,

$$
\phi=-\frac{a^{2}}{r} U_{0} \frac{x}{r} \cos \omega t=K \frac{x}{\left(x^{2}+y^{2}\right)},
$$

where $K=-a^{2} U_{0} \cos \omega t, a$ is the radius of the cylinder, and $U_{0} \cos (\omega t)$ was previously defined as the velocity amplitude of the cylinder motion. The relation $x=r \cos \theta$ was used to remove the $\cos \theta$ term of equation 2.3, and the other coordinate transformation is $y=r \sin \theta$, and $r^{2}=x^{2}+y^{2}$. A wall along the $\mathrm{x}$-axis may be formed by superposing these two cylinders at a distance of $d$ and $-d$ away from the wall axis,

$$
\phi_{\text {cyl-wall }}(x, y)=\phi(x, y-d)+\phi(x, y+d) \text {. }
$$

When evaluating $\phi_{\text {cyl-wall }}$ at the wall, $y=0$, then $\phi(x,-d)=\phi(x,+d)$, and the magnitude is doubled, $\phi_{\text {cyl-wall }}(x, 0)=\left.2 \phi\right|_{y=0}$. Equation 2.9 is not the exact solution, since only the boundary condition at the wall is satisfied, but not the boundary condition at the cylinder. The presence of the image cylinder will create a flow field that should be altered by the presence of the cylinder. In order to enforce the no-flux condition on the surface of the cylinder, an image cylinder needs to be placed within the cylinder. This new condition needs to be reflected on the other side of the wall boundary. These recursive effects are clearly neglected in equation 2.9, and would require additional $b_{i} \phi_{i}$ terms to solve for the exact solution. However, these recursive 
effects become less significant the further the cylinder and image cylinder are located from each other, and for the cases considered in this thesis, are neglected.

When the axis of oscillation is at a counterclockwise angle $\alpha$ relative to the wall, and its image is at a counterclockwise angle of $-\alpha$ relative to the wall, equation 2.9 can be modified as $\phi_{\text {cyl-wall }}(x, y, \alpha)=\phi(x, y-d, \alpha)+\phi(x, y+d,-\alpha)$, and the coordinates for the cylinder and its image undergo a rotational transformation of $\alpha$ and $-\alpha$, respectively. The result is

$$
\phi_{\text {cyl-wall }}(x, y, d, \alpha)=\mathrm{K}\left[\frac{x \cos \alpha-(y-d) \sin \alpha}{x^{2}+(y-d)^{2}}+\frac{x \cos (-\alpha)-(y+d) \sin (-\alpha)}{x^{2}+(y+d)^{2}}\right] .
$$

The pressure along the wall is

$$
p_{\mathrm{wall}}(x, 0, d, \alpha)=\kappa_{p}\left[\frac{x \cos \alpha+d \sin \alpha}{x^{2}+d^{2}}\right]
$$

where the constant $\kappa_{p}=2 \rho \omega U_{0} a^{2} \sin (\omega t)$. The gradient of the potential function is required to find the velocity throughout the fluid, whose components in cartesian coordinates are

$$
\begin{gathered}
V_{x}=\frac{\partial \phi_{\text {cyl-wall }}}{\partial x}=\mathrm{K}\left[\frac{\cos \alpha}{x^{2}+(y-d)^{2}}-\frac{2 x^{2} \cos \alpha-2 x(y-d) \sin \alpha}{\left(x^{2}+(y-d)^{2}\right)^{2}}\right] \\
+\mathrm{K}\left[\frac{\cos \alpha}{x^{2}+(y+d)^{2}}-\frac{2 x^{2} \cos \alpha+2 x(y+d) \sin \alpha}{\left(x^{2}+(y+d)^{2}\right)^{2}}\right],
\end{gathered}
$$

and

$$
\begin{gathered}
V_{y}=\frac{\partial \phi_{\text {cyl-wall }}}{\partial y}=\mathrm{K}\left[\frac{-\sin \alpha}{x^{2}+(y-d)^{2}}-\frac{2 x(y-d) \cos \alpha-2(y-d)^{2} \sin \alpha}{\left(x^{2}+(y-d)^{2}\right)^{2}}\right] \\
+\mathrm{K}\left[\frac{\sin \alpha}{x^{2}+(y+d)^{2}}-\frac{2 x(y+d) \cos \alpha+2(y+d)^{2} \sin \alpha}{\left(x^{2}+(y+d)^{2}\right)^{2}}\right] .
\end{gathered}
$$

At the wall, $V_{y}$ is zero as expected, and $V_{x}$ is nonzero, which is in keeping with an inviscid solution. The vertical component of velocity within the boundary layer is nonzero, and can now be predicted by equation 2.13. The wall-parallel component 
predicted by equation 2.12 does not change much throughout the boundary layer, and can be used to predict the fluid flow just outside of the viscous boundary layer, $U_{\infty}$

\subsubsection{D dipole next to fish-like bodies}

To explore the effects of body curvature on the received dipole signal, a smooth shaped fish is compared with cross-sections of an actual fish. To represent the smooth fish shape, a NACA 0020 profile was used, with its width matching the width of the other fish models. The NACA profiles are a series of experimentally derived airfoil shapes that have good lift and drag properties. The four digits are the inputs to an equation which generates its specific shape (Jacobs, Ward, and Pinkerton, 1932; Abbott and Von Doenhoff, 1959). The dimensions of a real fish body were taken from the crosssection of a Lake Michigan Mottled Sculpin, at the halfway height of the body. This gave a length of $7.6 \mathrm{~cm}$ and width of $2 \mathrm{~cm}$. A cross-section of the pectoral fins gave a thin slice of approximately $1.5 \mathrm{~cm}$ in length at roughly an angle of 45 degrees away from the body (see figure 2-3).

\section{Numerical set up}

For the coarse grid mesh, points around each body $2 \mathrm{~mm}$ apart were mapped to a grid. For a $50 \mathrm{~Hz}$ signal, the boundary layer is $\delta \approx 80 \mu \mathrm{m}$. With the base length, $b$, of each triangular mesh cell equal to the node spacing, the ratio $b / \delta \approx 25$ is similar to the flat wall case. An elliptical region circumscribed the fish (and fins), with nodes $2 \mathrm{~mm}$ apart around the perimeter. A cylinder $6 \mathrm{~mm}$ in radius was placed $5 \mathrm{~cm}$ away from the longitudinal midline of the fish, at about a distance of half way between the nose and tail of the fish. It had 20 nodes placed around its perimeter (approximately $2 \mathrm{~mm}$ apart), giving a node spacing to curvature ratio of approximately 0.3 and $b / \delta \approx 24.6$. The cylinder was surrounded by a deforming region 4 times its radius, with nodes spaced $2 \mathrm{~mm}$ apart around the perimeter. The exterior region was $40 \mathrm{x} 40 \mathrm{~cm}$, with nodes spaced $2 \mathrm{~mm}$ apart. All interior regions were evenly meshed with triangular 
Zoomed in views of the meshing for the NACA 0020 and sculpin with fins retracted/extended bodies

NACA 0020

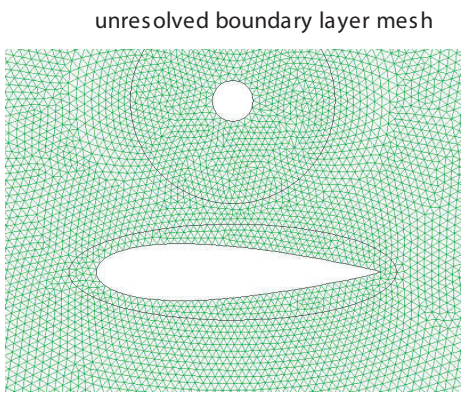

\section{Sculpin with fins retracted}

unres olved boundary layer mesh

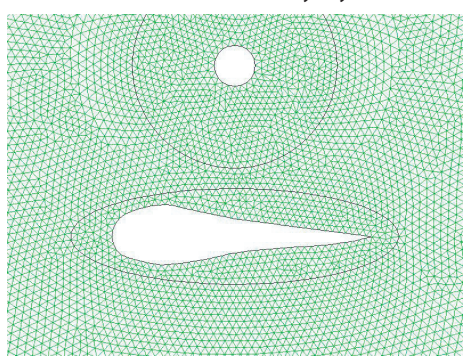

Sculpin with fins extended

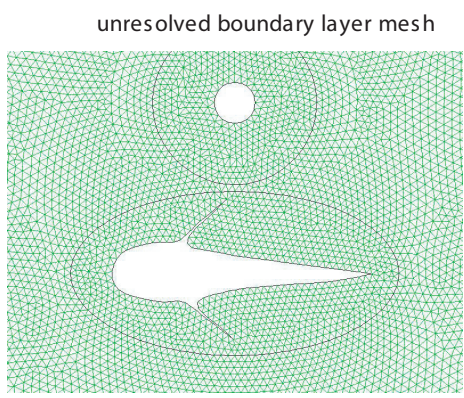

resolved boundary layer mesh

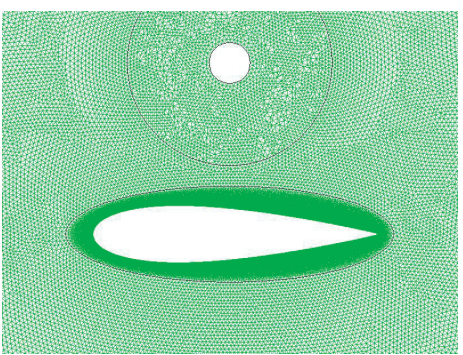

resolved boundary layer mesh

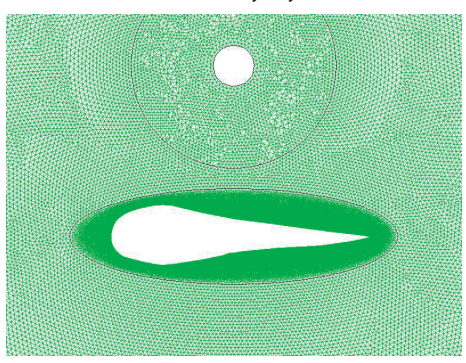

resolved boundary layer mesh

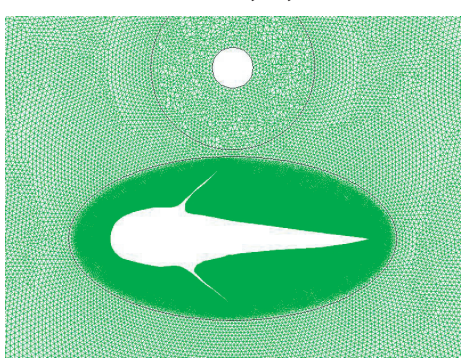

boundary layer around tail tip

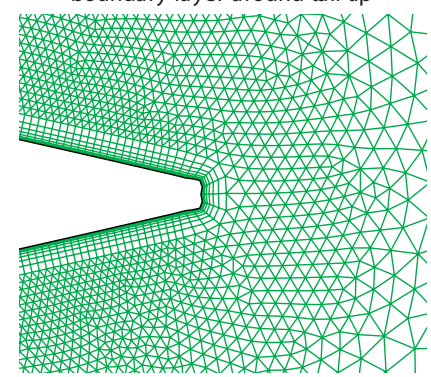

boundary layer around tail tip

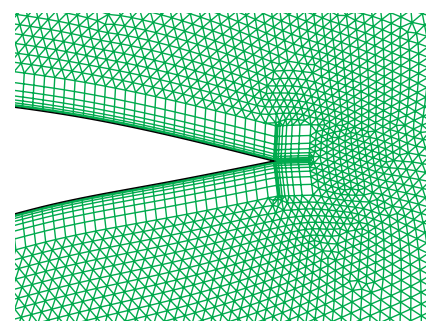

boundary layer around fin tip

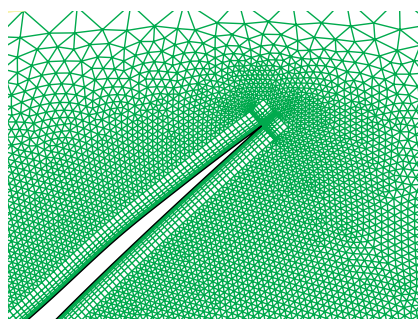

Figure 2-3: The grid domain for an unresolved surface boundary layer ('coarse' grid) and resolved surface boundary layer around various fish bodies. The coarse grid provides the 'potential flow' solution, since it has nodes in the inviscid region, whereas the resolved boundary layer grid has nodes in the viscous layer next to the wall. The cylinders have their centers located at a distance of 8.33 radii from the longitudinal midline of the fish. The NACA 0020 profile is one of a series of NACA profiles, which are airfoil shapes that NASA scientists discovered had good lift and drag properties. The 4 digits are inputs to empirically derived equations which dictate the shape of the airfoil (Jacobs, Ward, and Pinkerton, 1932). 
cells of uniform size.

For the resolved grid mesh, nodes were spaced evenly at $100 \mu \mathrm{m}(1.25 \delta)$ apart around the perimeter of the fish bodies. The height of the first node above the surface was $10 \mu \mathrm{m}(\delta / 8)$, and subsequent nodes were located at intervals of 1.4 times the previous interval for a total of 8 layers. An elliptical region large enough for the fish (and fins) to reside in was created to force the highly resolved region to come to a coarse meshing relatively quickly. Nodes $1 \mathrm{~mm}$ apart were placed around this perimeter, and the interior meshed with triangular cells. A cylinder of $6 \mathrm{~mm}$ in radius was placed at the same location as in the coarse grid mesh, but nodes were placed at $1 \mathrm{~mm}$ intervals around its perimeter, giving a node spacing to curvature ratio of 0.17 and $b / \delta \approx 12.5$. The cylinder was surrounded by a deforming region 4 times its radius, with nodes spaced $1 \mathrm{~mm}$ apart around the perimeter. The exterior region was 40x40 cm, with nodes spaced $1 \mathrm{~mm}$ apart. The region between the ellipse and outer boundaries was evenly meshed with triangular cells.

The fish and cylinder surface boundaries were given no-flux and no-slip boundary conditions, corresponding to velocity components being zero. One side boundary was given a zero pressure condition, the opposite side was given a zero velocity condition, and the final two sides were assigned symmetry boundary conditions. This combination of boundary conditions minimizes the impact that finite boundary locations have on the flow field generated by the oscillating cylinder. The cylinder velocity was set to $U_{0} \cos (\omega t)$ along the axis of vibration, with $U_{0}=7 \mathrm{~mm} / \mathrm{s}$, and the frequency of oscillation $(\omega=2 \pi f)$ set to $f=50 \mathrm{~Hz}$. FLUENT ${ }^{\mathrm{TM}}$ solved the unsteady simulations with a time step of $f / 100$, where $f=50 \mathrm{~Hz}$ was the frequency of oscillation.

\subsubsection{Changing dipole locations and orientations study}

Most studies consider the relevant hydrodynamic signals while neglecting the presence of the receiving body. However, both the presence of the body, and any protruding parts, alters the local hydrodynamic field. For the biologist, it is important to know what signal the animal is responding to, and for the engineer writing pattern recognition programs, distortions need to be addressed. Using computational fluid dynamics 
(CFD), 2D simulations are computed for a dipole source at various distances and orientations in relation to both a streamlined body profile and a body with extended fins. This scenario is commonly found in the literature (Coombs et. al, 1996; Coombs and Conley, 1997b; Curcic-Blake and van Netten, 2006; Yang et. al, 2006), and can represent a small prey moving in a variety directions relative to the side of the fish.

A dipole source will produce a signature spatial gradient along the lateral line, providing information about the location of the source relative to the body (CurcicBlake and van Netten, 2006). Taking the gradient of pressure in the direction parallel to the wall, equation 2.11 becomes

$$
\left.\frac{\partial p_{\text {wall }}(x, y, d, \alpha)}{\partial x}\right|_{y=0}=\kappa_{p}\left[\frac{\cos \alpha}{x^{2}+d^{2}}-\frac{2 x^{2} \cos \alpha}{\left(x^{2}+d^{2}\right)^{2}}-\frac{2 x d \sin \alpha}{\left(x^{2}+d^{2}\right)^{2}}\right]
$$

where $\kappa_{p}=2 \rho \omega U_{0} a^{2} \sin (\omega t)$, and $d$ is the distance the cylinder was placed away from the wall. When $\alpha=0$, the zero-crossings (or nulls) occur when $x= \pm d$, and the maximum value occurs at $x=0$. Therefore, zero crossings encode the distance of the cylinder, and the location of the peak along the wall encodes the location of the cylinder along the wall (i.e. it is the perpendicular bisector of the wall, drawn through the center of the cylinder).

In a similar manner, when $\alpha=\pi / 2$, the zero-crossing is now the location of the perpendicular bisector, and the cylinder distance can be found by setting the derivative of the pressure gradient equal to zero. This relation occurs when the pressure gradient is at either of its two peaks, $x= \pm d / \sqrt{3}$. Figure 2-4 shows the plots for the pressure gradient when a cylinder oscillates at angles of $0,30,60,90$ degrees relative to the x-axis. Using the spatial encoding clues, the plot reveals that the cylinder is located on the y-axis at a distance of 10 units away.

For the numerical simulations, a cylinder $3 \mathrm{~mm}$ in radius was placed at either 3 $\mathrm{cm}$ or $5 \mathrm{~cm}$ away from the longitudinal midline of the fish body, in locations ranging from the front to the tail of the fish. The coarse grid meshing around the cylinder and fish bodies has already been described. The nodes around the fish bodies were at $2 \mathrm{~mm}$ intervals, and from these points three sections were selected to represent 


\section{Dipole pressure gradient patterns on a surface due to a cylinder oscillating between 0-90 degrees}
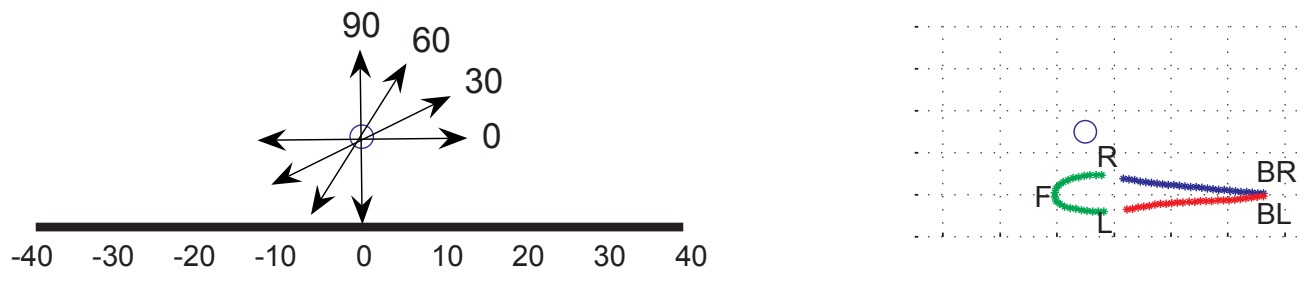

Pressure gradient signal along a wall

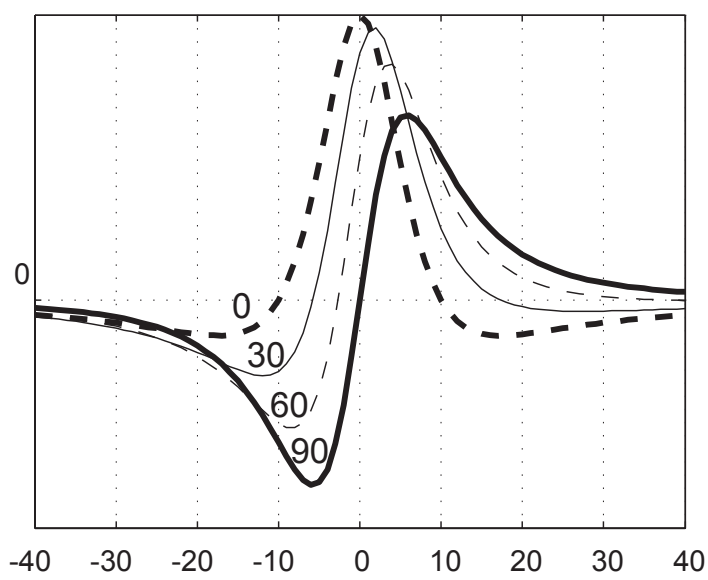

Pressure gradient signal around a sculpin

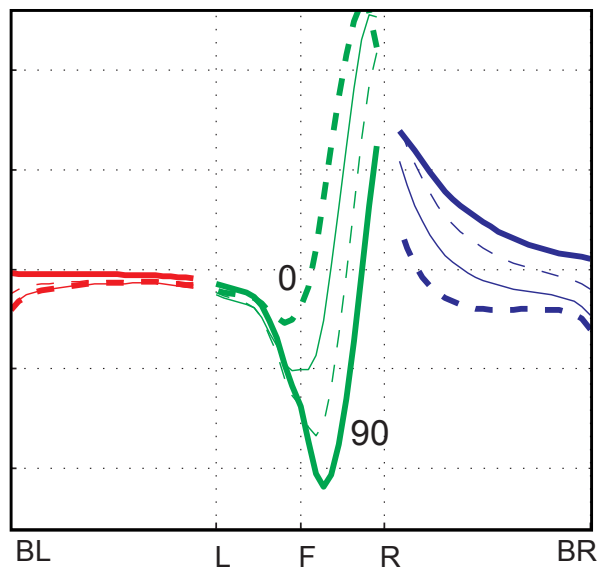

Pressure differencing defined

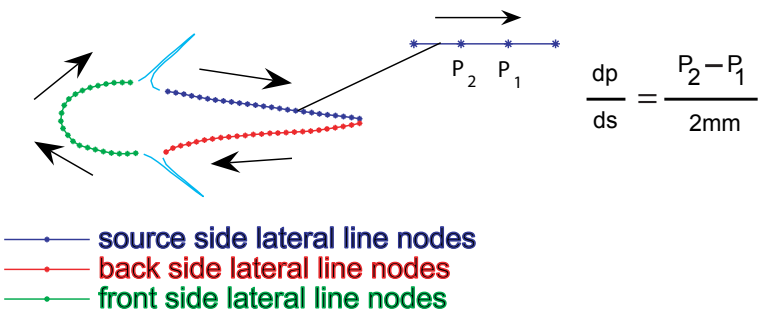

Figure 2-4: Pressure gradient patterns on a wall due to an oscillating cylinder. As the axis of oscillation is varied between 0-90 degrees, pressure gradient patterns specific to each angle appears on the surface of a wall, and also on the surface of the canal lateral line of fish. The locations of peaks and zero crossings uniquely encodes information about the location of the cylinder (see text). The pressure gradient is really a pressure difference between canal lateral line pore openings, whose direction is assigned to show continuity over the whole lateral line. 
idealized pore openings on a left, front, and right canal lateral line. The pressure difference between pore openings was calculated according to $\left(p_{2}-p_{1}\right) / 2 \mathrm{~mm}$ (see figure 2-4).

The boundaries of these simulations were $375 \times 100 \mathrm{~cm}$, with nodes around the perimeter spaced at $10 \mathrm{~cm}$ intervals. Three sides were given a zero velocity boundary condition, and the fourth side a zero pressure boundary condition. Pressure needs to be specified at one side for the equations to be solved. The boundaries are located far enough away from the oscillating cylinder that the flow induced velocity at these boundaries is effectively zero. The cylinder velocity was set to $U_{0} \cos (\omega t)$ along the axis of vibration, with $U_{0}=1.6 \mathrm{~mm} / \mathrm{s}$, and the frequency of oscillation $(\omega=2 \pi f$ ) set to $f=25 \mathrm{~Hz}$. FLUENT ${ }^{\mathrm{TM}}$ solved the unsteady simulations with a time step of $f / 100$, were $f$ is the frequency of oscillation.

As equation 2.14 shows, results can be normalized by $\rho \omega U_{0} \sin (\omega t)$, leaving a dimensionless geometric term scaled by $(a / r)^{2}$. To confirm that the scaling did not affect results, simulations were also run with $U_{0}=1 \mathrm{~mm} / \mathrm{s}$ and $f=50 \mathrm{~Hz}$, leading to identical results (not shown).

\section{$2.3 \quad$ Results}

\subsubsection{Oscillating cylinder next to a wall}

The first goal of this chapter is to see if the boundary layer flow next to a wall from a nearby oscillating cylinder can be predicted. The idea is that potential flow theory can predict the general dipole flow field, including the wall-parallel flow outside the boundary layer and the vertical velocity inside the boundary layer. Secondly, although Stokes' solution for an oscillating boundary layer flow is based on a flow of uniform magnitude, $U_{\infty}$, it may be that allowing for a local velocity, $U_{\infty}(x)$, will work where the spatial changes of the velocity are gradual.

The second goal of this chapter is to see if a coarse grid representation of the flow will adequately recreate the potential flow solution. The advantage is a huge cost in 
computational time and memory for 3D cases (considered later). Both goals will be compared against a resolved boundary layer solution, which will be taken as the true solution.

Figure 2-5 shows the instantaneous velocity $($ phase $=0)$ and pressure $($ phase $=\pi / 2)$ along the wall for a cylinder oscillating in directions parallel and perpendicular to the wall. The locations for the velocity measurements from the coarse grid mesh are at the wall adjacent cell centroids, approximately $0.5 \mathrm{~mm}$ above the wall. The locations for the velocity measurements from both the potential flow calculations and from the resolved boundary layer mesh are at $0.55 \mathrm{~mm}$ above the wall. The locations for the pressure measurements for all results are located on the wall itself, since all methods are able to make these calculations directly.

The results show that all three methods produce almost identical results. For the wall-parallel velocity, $U_{\infty}(x)$, the coarse grid simulations give a slightly reduced magnitude. This is not unexpected, since the coarse grid simulations are still viscous simulations, and FLUENT ${ }^{\mathrm{TM}}$ interpolates the cell center value from the corner nodes that form the cell. Secondly, the potential flow calculations also differ just slightly from the resolved grid simulations. As mentioned earlier, there are some real effects not accounted for using the potential flow approximation of a cylinder and its image, without performing recursive calculations.

The pressure along the wall shows exact agreement between the coarse grid and resolved grid boundary layer simulations. However, the discrepancy with potential flow theory in the case of the cylinder oscillating parallel to the wall is due to boundary effects. The simulations have boundaries at finite distances, and the results reveal that the boundaries were not placed far enough away from the cylinder. The potential flow theory has its side and top boundaries at infinity. As such, the potential flow results were shifted so that the pressure at the side boundary was forced to be zero, similar to the numerical simulation boundary conditions. If the boundaries in the simulations were extended, there would be exact overlap of all solutions. The reason that this effect does not show up for the velocity plots is that velocity decays much faster at $1 / r^{2}$, whereas pressure decays at $1 / r$. 

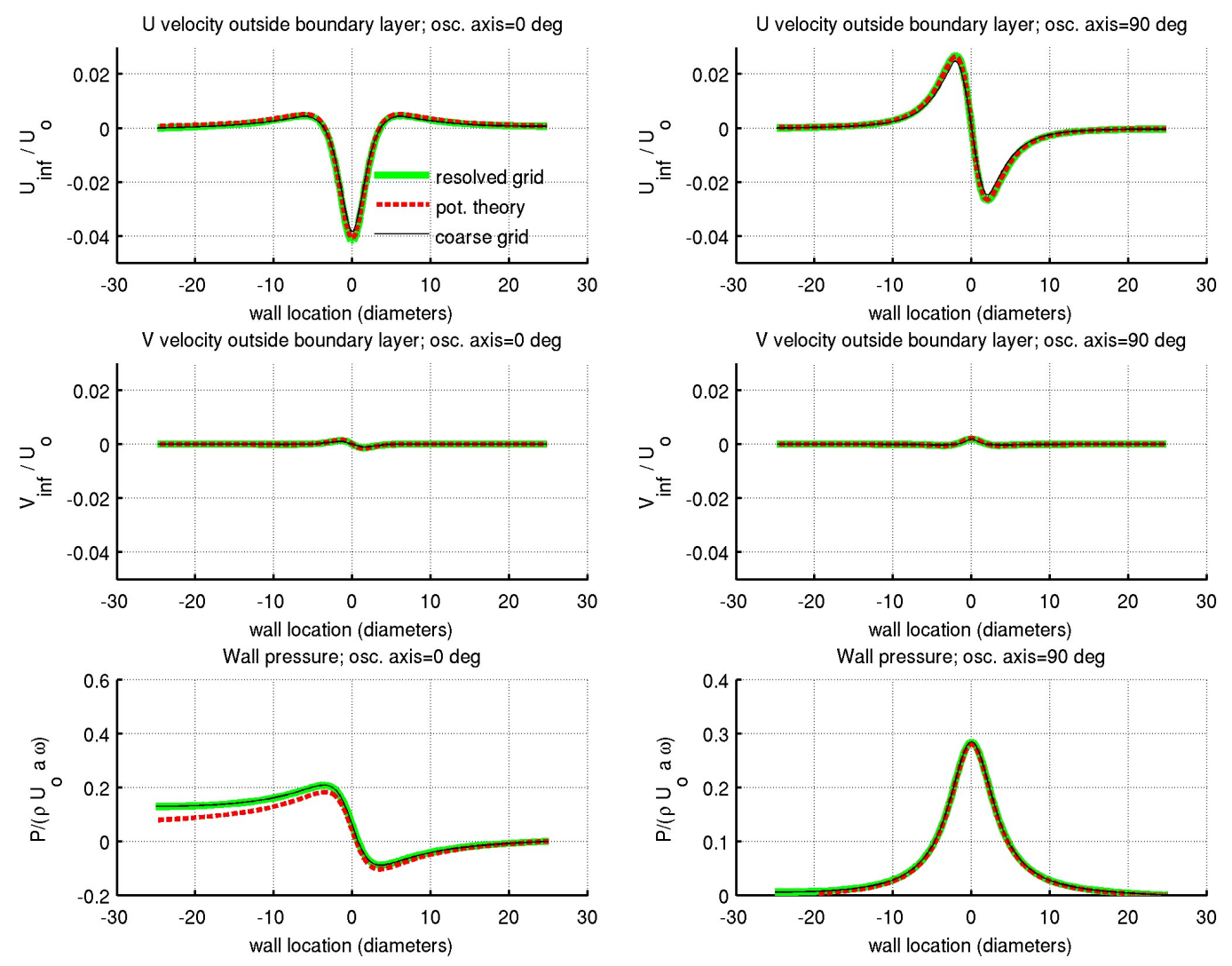

Figure 2-5: Instantaneous velocity $($ phase $=0)$ and pressure $($ phase $=\pi / 2)$ along the wall due to cylinder oscillating in parallel and perpendicular directions. Three methods are compared: a resolved boundary layer numerical solution, a coarse grid (unresolved boundary layer) numerical solution, and a potential flow theory solution. The wall-parallel velocity $\left(U_{\infty}(x)\right)$ and the vertical velocity $\left(V_{\infty}(x)\right)$ were measure at a distance of $0.5 \mathrm{~mm}$ away from the wall, which should be outside the boundary layer created by $50 \mathrm{~Hz}$ oscillations (see text). The pressure is measure at the wall. The dimensional values are scaled by the cylinder parameters: $U_{0}$, the velocity amplitude; $a$, the radius; $\omega$, the radian frequency of oscillation. $\rho$ is the density of water. The velocity and pressure patterns reflect the location and orientation of the cylinder, which is located at distance of $d=7 a$. 
Figures 2-6 and 2-7 show the velocity field around the cylinder and velocity profiles within the boundary layer when the cylinder oscillates parallel and perpendicular to the wall. The plots are colored by velocity magnitude, and vectors are of uniform length to better reveal the flow patterns. The boundary layer profiles compare the resolved boundary layer simulation results to those of the potential flow theory and oscillating boundary layer flow approximation results. The coarse grid results are not presented, as they were just shown to be in agreement with the potential flow theory results. Also, the coarse grid simulations cannot predict the vertical velocity in the boundary layer like the potential flow theory is able to do. The potential flow calculations for $U_{\infty}(x)$ were made at $1.5 \mathrm{~mm}$ ( $18 \delta$ ) above the wall, whereas the calculations for the vertical velocity were made at the exact same locations as the resolved boundary layer simulations.

The fluid velocity field in figure 2-6 shows the effect of the wall in forcing the flow to move parallel to it. The uniform length vectors help the eye to trace streamlines, and one such set of streamlines shows how the wall redirects the flow from its intended path around the cylinder. Instead, the streamlines diverge along the wall, and at their juncture, a stagnation point, or low velocity region exists. According to the boundary layer velocity profiles presented (figure 2-6,C), this is the region that is most poorly predicted by potential flow theory and boundary layer approximation. However, in comparison to other regions of flow along the wall, the velocities at the stagnation point are orders of magnitude less, and that is its significance to the fish lateral line.

The boundary layer velocity profiles of figure 2-6 show that potential flow theory, used with the approximation to Stokes oscillating boundary layer, match the numerical simulation results quite well. Discrepancies that appear, especially when the phase is $\pi / 2$ and the vertical velocity should be zero according to potential flow theory and according to Stokes solution, show the real effects of a dipole induced boundary layer. It should be noted that the vertical velocity, in comparison to the wall-parallel velocity, is almost always an order of magnitude smaller, if not more. Therefore, the dominant stimulus to the superficial neuromast is well predicted.

Figure 2-7 shows results when the cylinder is oscillating perpendicular to the wall. 
Instantaneous velocity field and boundary layer for a cylinder oscillating parallel to a wall

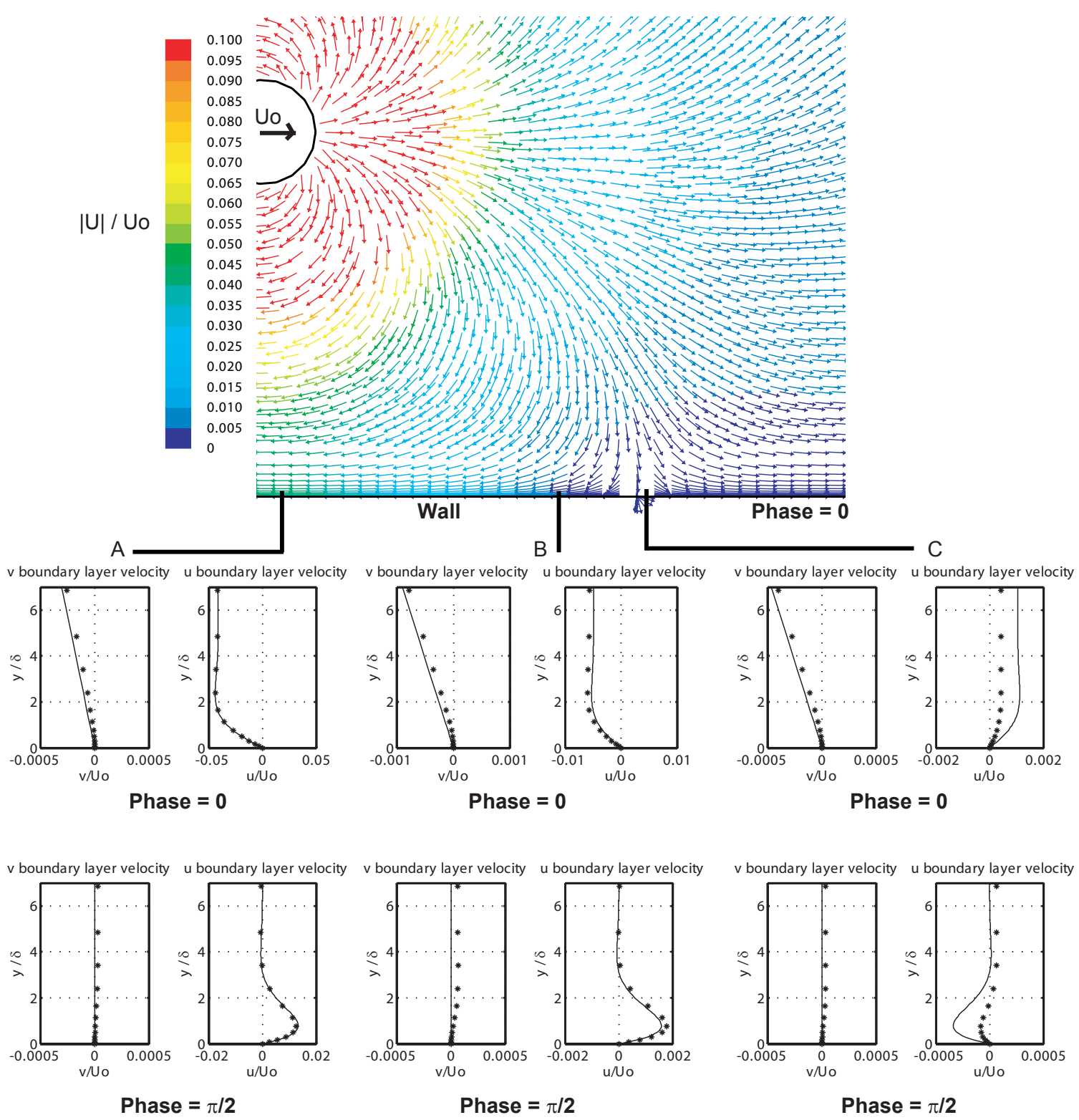

Figure 2-6: Velocity field (at phase=0) and boundary layer profiles for a cylinder oscillating parallel to a wall. Vectors of uniform length represent the direction of the flow, while colors indicate the velocity magnitude. Representative velocity profiles in the boundary layer are shown at phase $=0$ and also at phase $=\pi / 2$. The solid lines are the predicted values using potential flow theory and Stokes' oscillatory flow next to a plate. The data points are from the resolved boundary layer numerical simulations. 
Instantaneous velocity field and boundary layer for a cylinder oscillating perpendicular to a wall
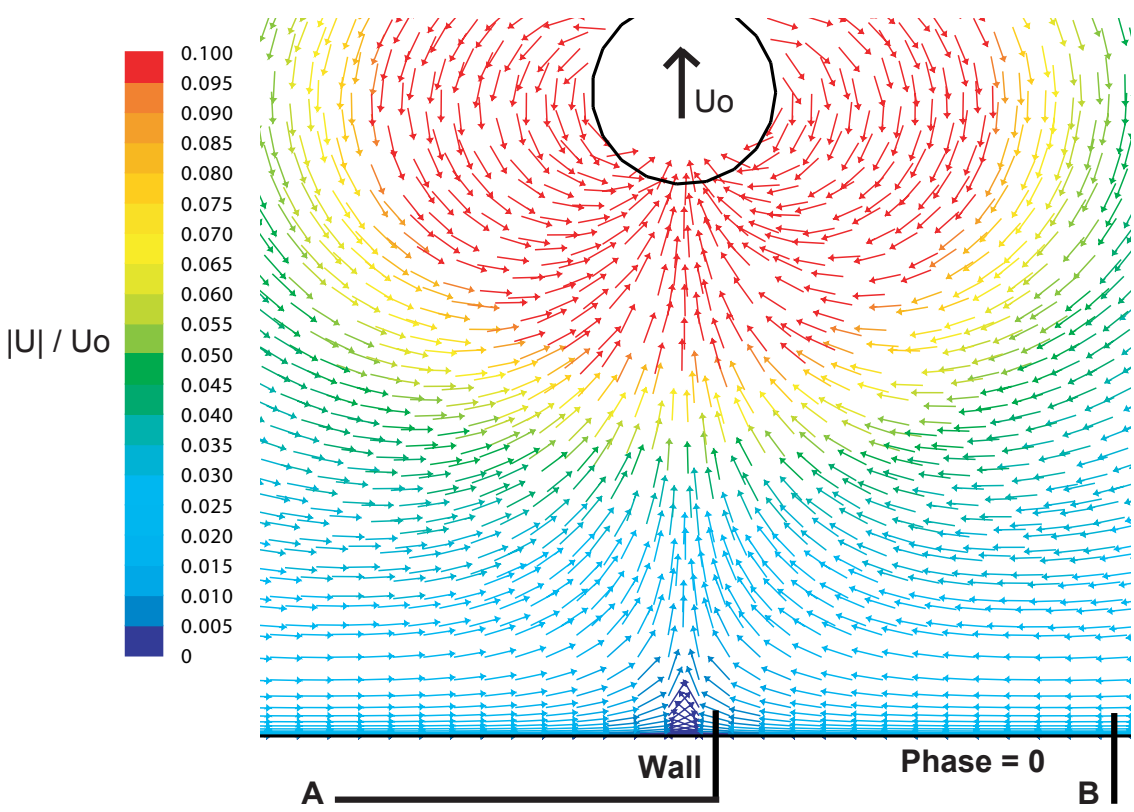

$v$ boundary layer velocity $u$ boundary layer velocity
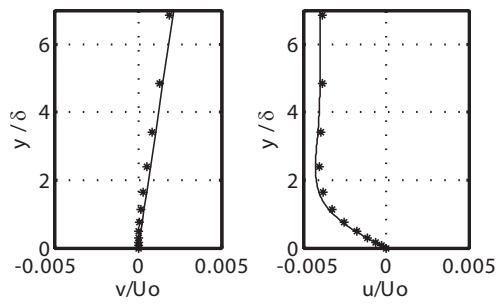

Phase $=0$
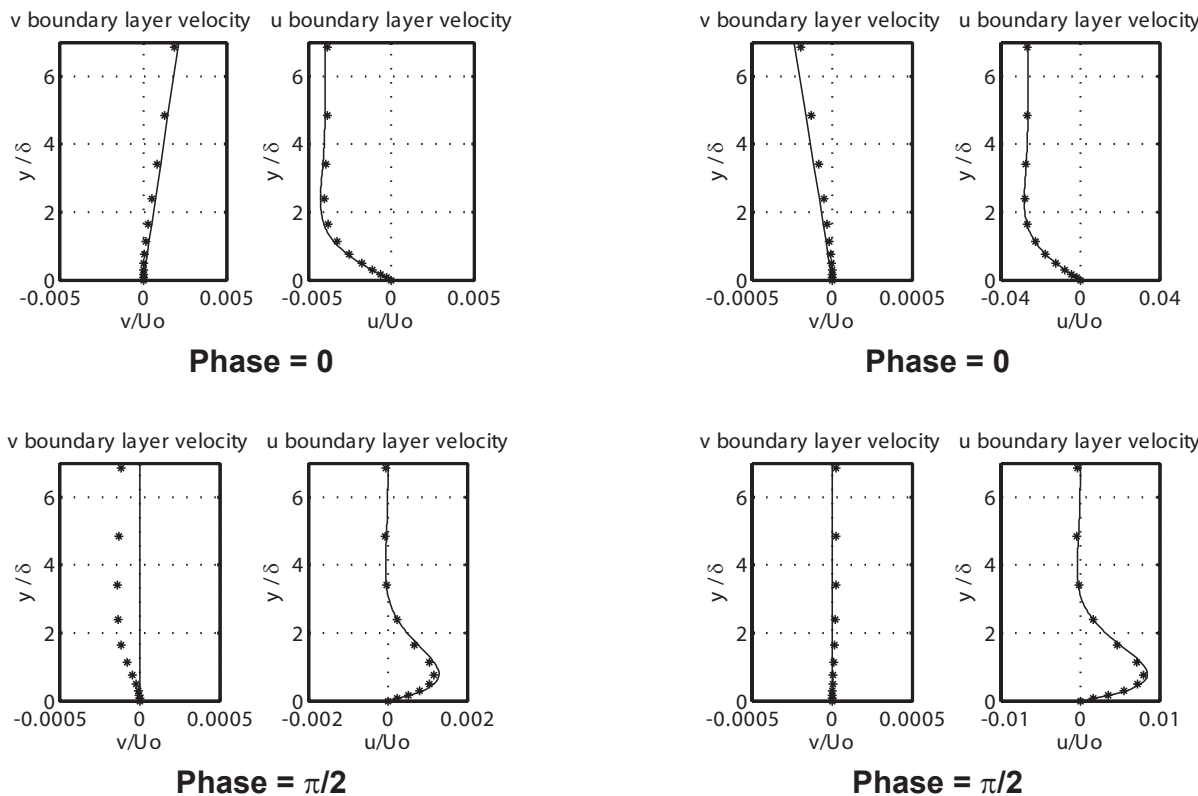

Figure 2-7: Velocity field (at phase=0) and boundary layer profiles for a cylinder oscillating parallel to a wall. Vectors of uniform length represent the direction of the flow, while colors indicate the velocity magnitude. Representative velocity profiles in the boundary layer are shown at phase $=0$ and also at phase $=\pi / 2$. The solid lines are the predicted values using potential flow theory and Stokes' oscillatory flow next to a plate. The data points are from the resolved boundary layer numerical simulations. 
Again the flow is forced parallel to the wall. There is one stagnation point, and the wall-parallel flow is well predicted in its vicinity (unlike the previous case). Together, these results show that for making predictions of boundary layer flow along a flat surface, the theory and approximations work quite well over a large portion of the surface.

\subsubsection{Oscillating cylinder next to fish bodies}

The second goal of this chapter is to compare the changes to the pressure and velocity patterns that result when changing from a flat wall surface to that of fish body shapes. The flat wall is effectively of infinite length, whereas the fish bodies are of finite dimension, and the flow can move around the fish. Secondly, the wall is a flat surface, with well predicted pressure and velocity patterns from an oscillating cylinder. The fish bodies have more curvature, which should introduce uneven movement of the water past the surface, creating localized uneven flow accelerations and pressure changes.

Figure 2-8 shows instantaneous velocity (phase $=0)$ and pressure $($ phase $=\pi / 2)$ patterns from an oscillating sphere located to the side (see figure 2-3) of the NACA 0020 shaped fish body. Results using the coarse grid numerical simulation are compared with results from the resolved boundary layer numerical simulations. Here, the coarse grid solution represents a close approximation to the potential flow solution, though this solution does include a no-slip boundary condition. The velocity is measured outside the assumed boundary layer, at a mean height of $0.6 \mathrm{~mm}$, while the pressure is measured at the body surface. The velocity components are decomposed into $U_{\infty}$ and $V_{\infty}$, which corresponds to the identical coordinate system of the flat wall case (figure 2-5).

In comparing velocities around the NACA 0020 fish body with those along the wall (figure 2-5), it should be noted that the stagnation point at the wall, which is directly beneath the cylinder, is now at the $0.47 \mathrm{BL}$ (body lengths) mark in figure 2-8. At that point, there is a similar peak for $U_{\infty}$ when the cylinder is oscillating parallel to the surfaces, and a similar zero crossing point for $U_{\infty}$ when the cylinder is 
oscillating perpendicular to the surfaces. Note that the scale has changed to surface body lengths, where $1 \mathrm{BL}$ for a NACA profile is approximately 7 diameters, and '0' corresponds to the nose, '1' to the source side tail point, and '-1' to the away side tail point. In comparison to the wall surface plots, these fish surface plots are 'zoomed' in.

When the cylinder is oscillating parallel to the fish, figure 2-8 shows the zero crossings for $U_{\infty}$ are at 0.17 and $0.83 \mathrm{BL}$, which is leads to uneven distances from the peak location. The distance between zero crossings is $0.66 \mathrm{BL}$ (4.6diameters), with the cylinder 3.5 diameters away from the point on the fish directly beneath it. In comparison, figure 2-5 shows that the zero crossings for $U_{\infty}$ are at 3.6 and -3.6 diameters for a cylinder located 3.5 diameters away. The zero crossing distance has shortened for the side of the fish in comparison with the flat wall surface results, and would falsely indicate the prey to be closer than actuality. The peak pressure is nearly the same in both cases, but the velocity overshoot is much larger for the fish (0.01$0.02 \mathrm{Uo}$ for the fish vs. $0.005 \mathrm{Uo}$ for the wall). The positive $U_{\infty}$ velocities at $1 \mathrm{BL}$, and negative $U_{\infty}$ velocities at $-1 \mathrm{BL}$, show that the flow is wrapping around the tail. At the nose $U_{\infty}$ goes to zero while $V_{\infty}$ is positive, which shows that the flow wraps around the nose. The distance between the pressure peaks matches the distance between zero crossings, just as it does for the flat wall case, and the magnitude difference between peaks is about $2 / 3$ that of the wall pressure difference.

When the cylinder is oscillating perpendicular to the side of the fish, figure 2-8 shows that magnitude of the $U_{\infty}$ at the peaks is similar to that of a flat wall, with the cylinder located 3.5 diameters away in each case. The distance between $U_{\infty}$ peaks is 3.6 diameters for the fish, and 4 diameters in the case of the wall, again falsely indicating a closer prey for the fish. At the tail and nose, the flow wraps around the fish, but opposite in direction to the parallel oscillating cylinder case. The pressure difference on either side of the fish at the peak is about $2 / 3$ of the peak pressure difference for the case of the wall.

Figure 2-9 shows that the cross section of a real sculpin body is a slight modification of the basic NACA 0020 shape, and leads only to slight changes in the velocity 
and pressure patterns on the surface. When the pectoral fins are extended, figure 2-10 shows that velocities behind the fin insertion points goes to zero. The flow around the pectoral fins has been omitted, which leaves discontinuities in velocity and pressure at the surface where the fins meet the body. The discontinuities in pressure highlight the locations of the insertion points, and show how the fins have the ability to 'dam' up the pressure on either side of the fin with the direction of the flow. The $V_{\infty}(d s)$ is very similar to that of the NACA 0020 fish body (figure 2-8), as is the $U_{\infty}(d s)$ for the case when the cylinder is oscillating perpendicular to the side of the fish. The flow is generally away from the body for the perpendicular oscillating cylinder, and as the fins also point away from the body, there is not much disruption. However, when the cylinder is oscillating parallel to the fish body, the $U_{\infty}(d s)$ is altered significantly in comparison to the NACA 0020 body (figure 2-8) and the sculpin with fins retracted profile (figure 2-9). The magnitude of the flow around the fins is overall reduced, as the fins work to block the direction of flow.

The flow patterns around the sculpin bodies are illustrated in figures 2-11, 212, 2-14, and 2-15. In addition, surface tangential, $u$, and normal velocities, $v$, are shown in the boundary layer next to the fish at selected locations. This is a change in coordinate system from the previous plots, which had a fixed coordinate system matching that of the flat wall set up $\left(U_{\infty}(d s)\right.$ and $\left.V_{\infty}(d s)\right)$. At any point of the fish surface, the positive directions are clockwise for $u$ and away for $v$. The prediction for the $u$ velocity profile is shown as the solid black lines, whereas all measurements from the resolved boundary layer numerical simulation are plotted as data points. The predicted $u$ profile uses the coarse grid solution $0.6 \mathrm{~mm}$ away from the boundary to get $u_{\infty}$, and then Stokes' solution for oscillating flow within the boundary layer. There is no prediction for $v$ within the boundary layer, since an analytical solution using potential flow theory was not used, and would be largely inadequate even if it was used.

Figure 2-11 shows the instantaneous velocity flow field around the sculpin body at phase $=0$. The vectors are of uniform length to show the direction of the flow, while the color indicates the magnitude. Visually, the impact of the body presence on the 

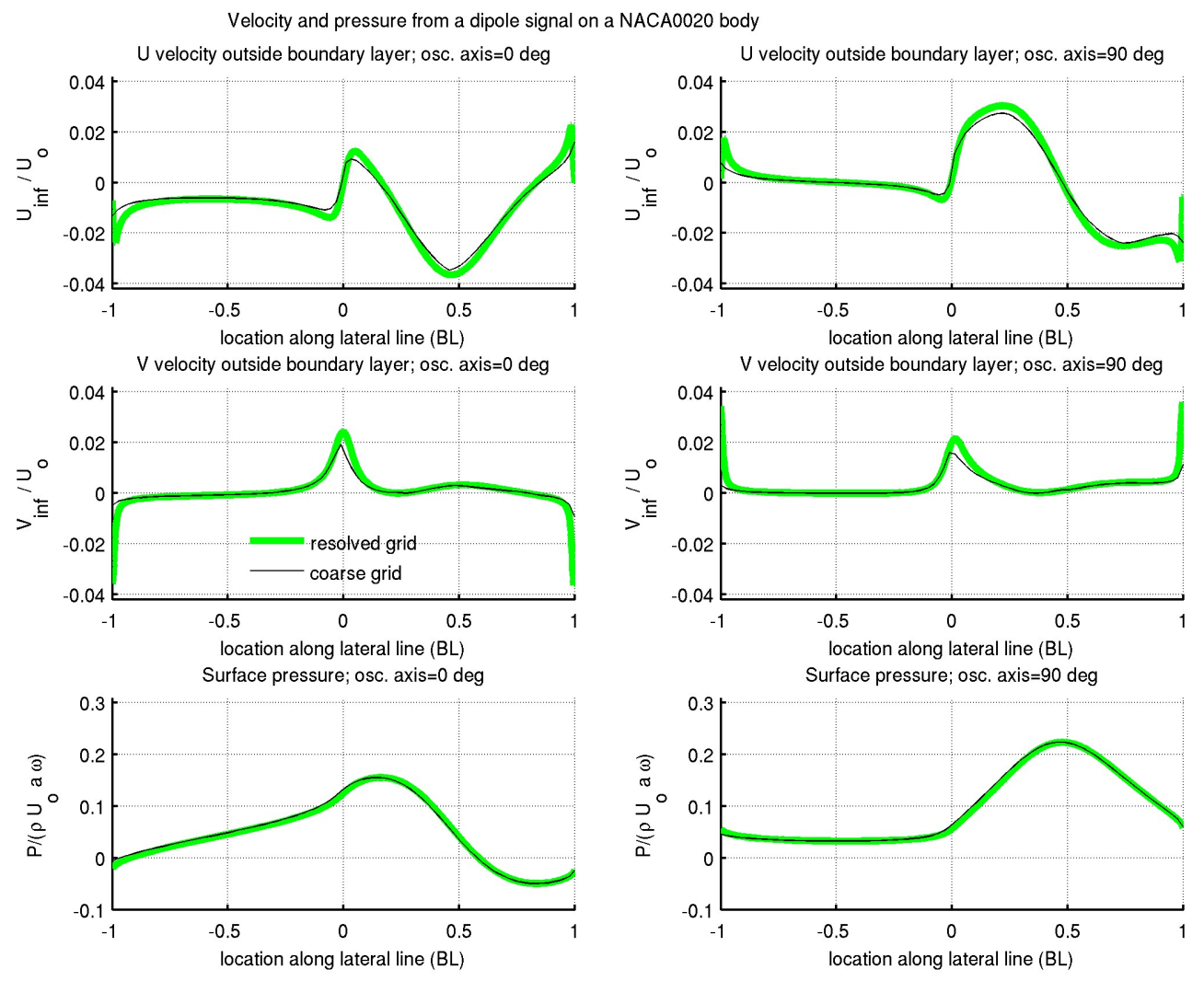

Figure 2-8: Instantaneous velocity $($ phase $=0$ ) and pressure $($ phase $=\pi / 2$ ) along a NACA 0020 shaped fish surface due to cylinder oscillating in parallel and perpendicular directions. The cylinder is located 3.5 diameters away from the point of the fish surface directly beneath it. A coarse grid numerical solution is compared with a resolved boundary layer numerical solution. The coordinate system matches the flat wall case for comparison purposes (see figure 2-5), with the exception that the $U_{\infty}(d s)$ and $V_{\infty}(d s)$ velocity components are located along the fish body surface (with units of surface body length, BL). The velocity measurements are made at $0.5 \mathrm{~mm}$ away from the body, while the pressure measurements are made at the body surface. 

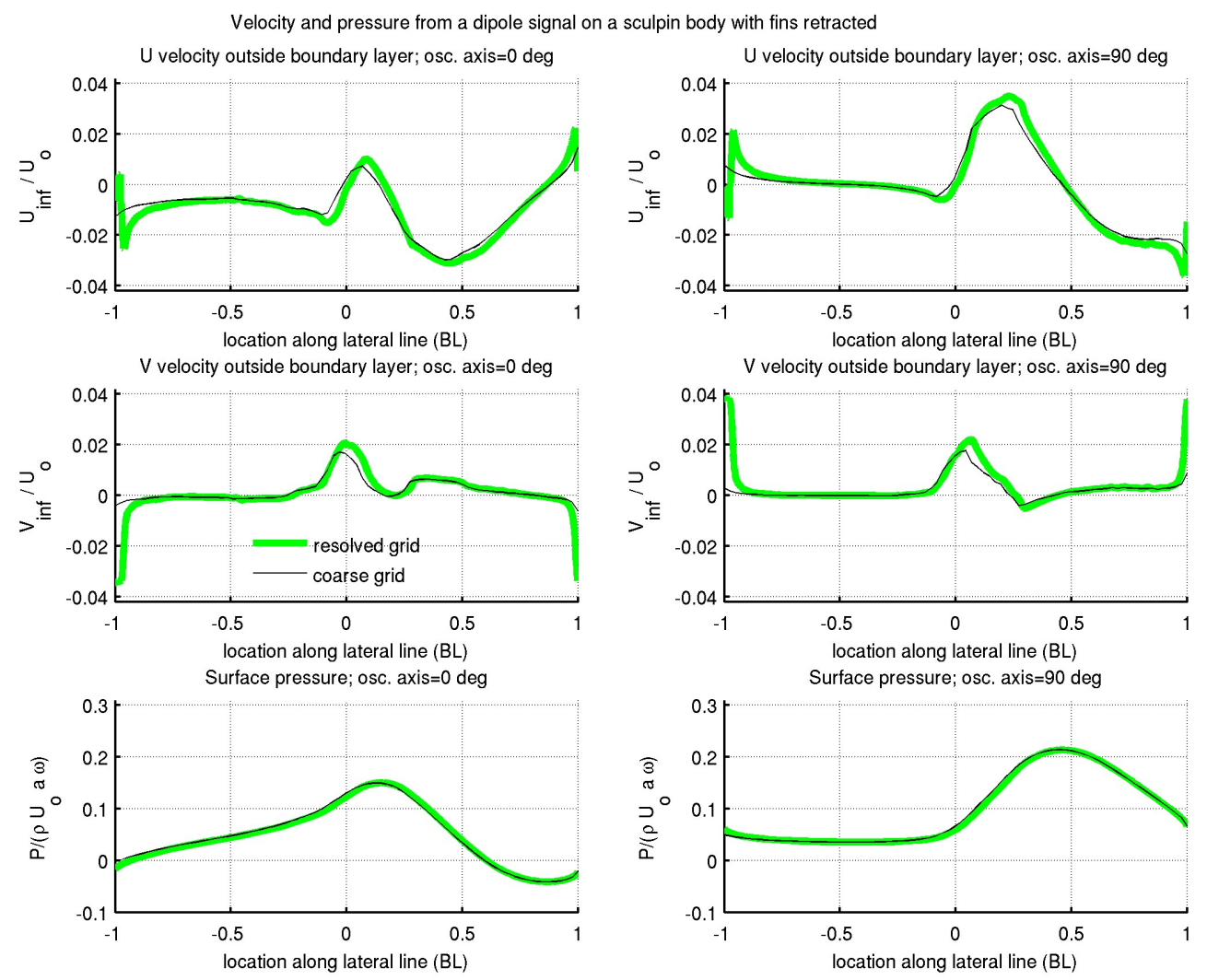

Figure 2-9: Instantaneous velocity $($ phase $=0)$ and pressure $($ phase $=\pi / 2)$ along the surface of a sculpin due to a cylinder oscillating in parallel and perpendicular directions. The cylinder is located 3.7 diameters above the point on the fish surface directly below it. Other parameters are as given in figure 2-8, which shows the NACA 0020 shaped fish body for comparison purposes. 

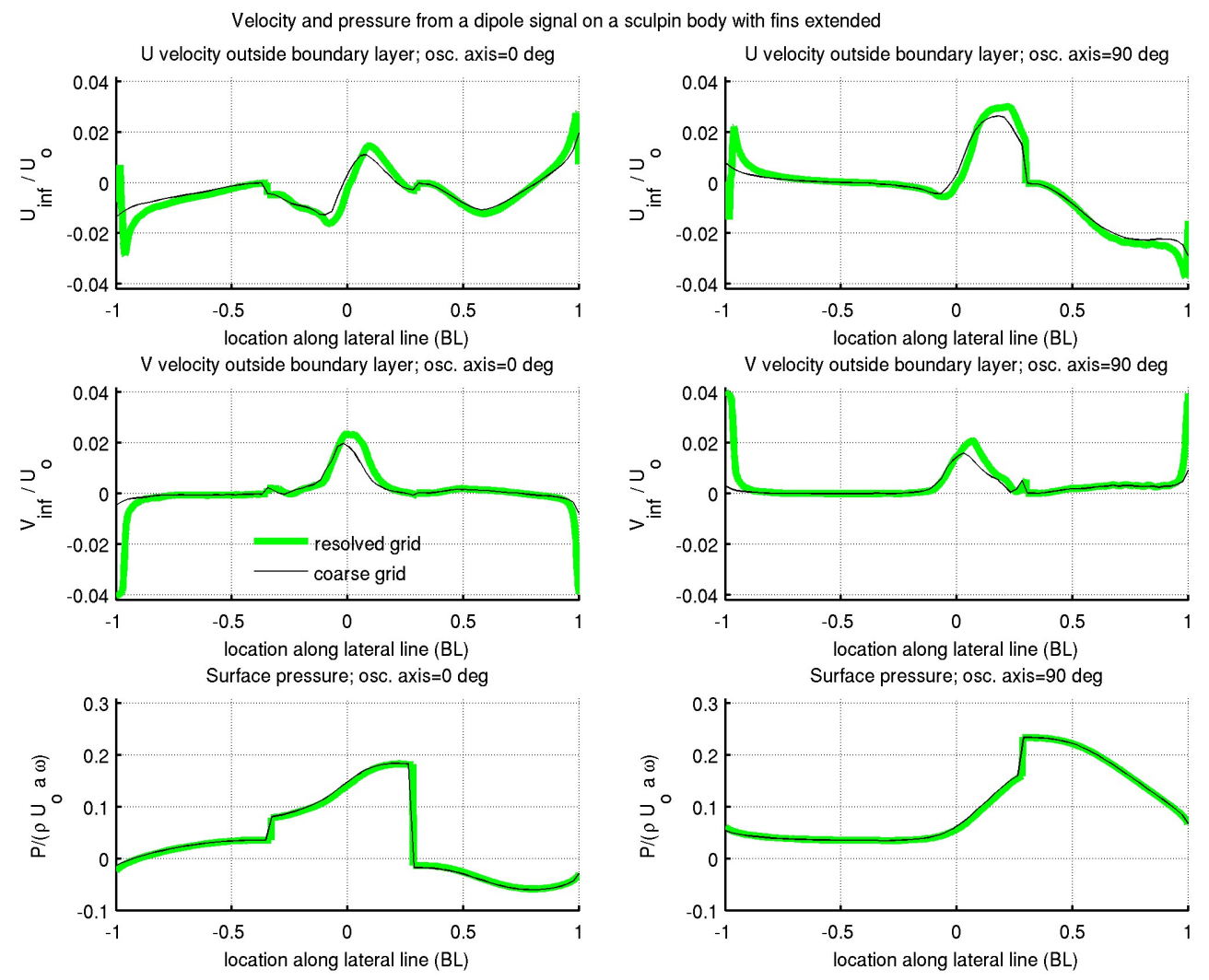

Figure 2-10: Instantaneous velocity $($ phase $=0)$ and pressure $($ phase $=\pi / 2)$ along the surface of a sculpin due to a cylinder oscillating in parallel and perpendicular directions. The cylinder is located 3.7 diameters above the point on the fish surface directly below it. Parameters are as given in figures 2-8 and 2-9, which shows the NACA 0020 shaped fish body and sculpin with fins retracted for comparison purposes. The flow around the pectoral fins has been omitted, leaving discontinuities on the surface of the sculpin at the fin insertion point. These are most obvious in the pressure plots (as vertical jumps), but can also be seen in the velocities at the same locations. 
flow can be seen as the otherwise concentric colors of decreasing velocity magnitude is altered to form a region of increased velocity next to the source side of the fish. Also apparent are the two stagnation points where the velocity magnitude decreases to almost zero above the surface. At the stagnation point near the tail, the flow is coming into the fish and is forced to split directions, and at the stagnation point on the head, the flow is coming together from opposite directions and is ejecting from the side of the fish. Within the boundary layer, $v$ is around an order of magnitude less than $u$ (at least), and shows that the predictions of $v$ for the flat wall case (see figure 2-6) would be largely in error in the majority of locations. This is not unexpected, as body curvature should create additional accelerations to those predicted by potential flow theory for flow near a flat wall. The predicted $u$ profile matches the actual results quite well at almost every location, except near the stagnation point where it underestimates the magnitude. The phase $=\pi / 2$ plots are shown in figure $2-13$ (top set of plots), and show even better agreement. The $v$ profiles show a variety of responses. Note: the scales change for every plot so that the velocity profiles are always 'zoomed in'.

When the cylinder oscillates perpendicular to the side of the sculpin (figure 212), the body presence also alters the flow field. The otherwise concentric circles of declining velocity magnitude show increased regions of flow near the head and tail. The stagnation point is located at the side of the fish at the point that is nearly beneath the cylinder. It is slightly offset in order to bring the stagnation point a little closer to the cylinder, and in line with the perpendicular bisector of the side of the fish (rather than the longitudinal midline of the fish). There is also a stagnation point on the opposite side of the fish (close to point E), where the flow splits and circulates around the fish body to the source side stagnation point, where the fluid is ejected. The shape of the $u$ velocity profiles within the boundary layer is well predicted, though the magnitudes are often slightly diminished. When phase $=\pi / 2$, as shown in figure 2-13 (bottom set of plots), the $u$ velocity profiles are very well predicted.

Figure 2-14 shows the instantaneous velocity flow field around the sculpin body 


\section{Instantaneous velocity field and boundary layer for a cylinder oscillating parallel to the fish}

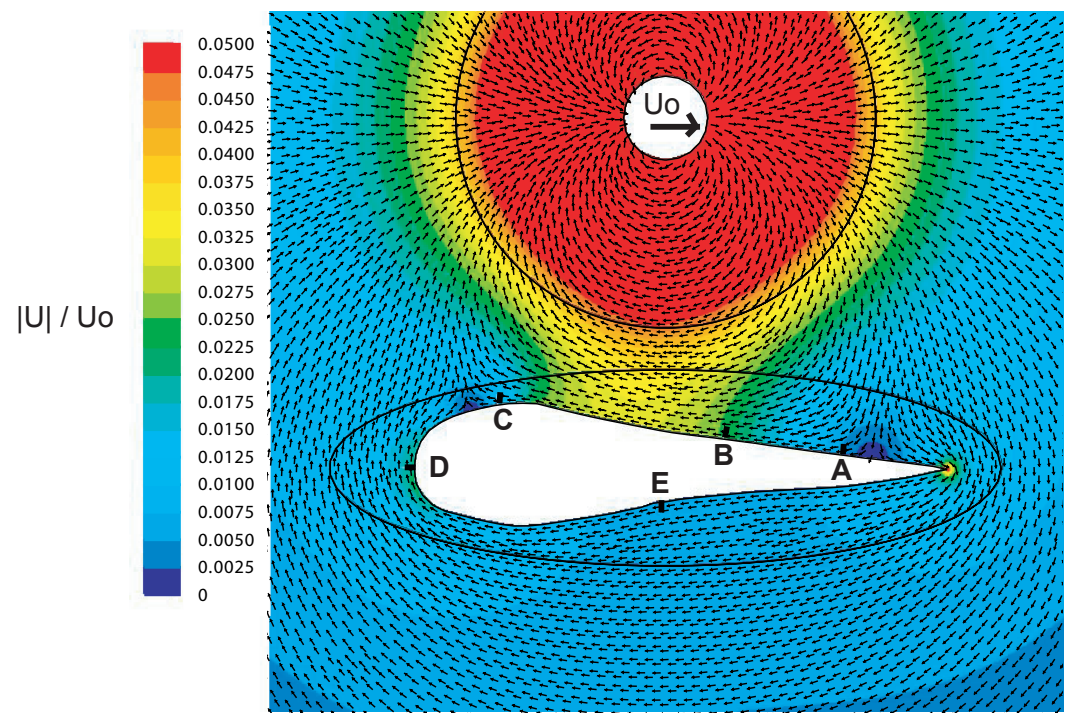

Phase $=0$
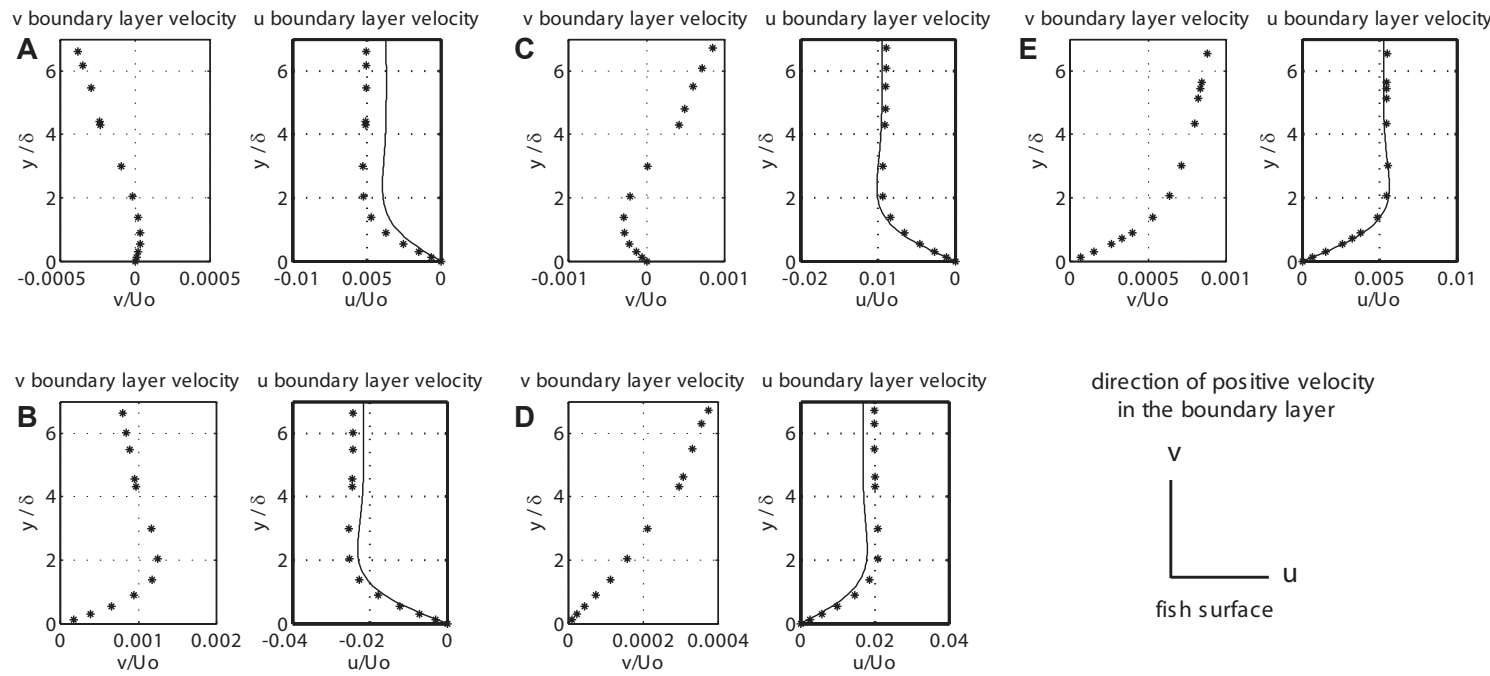

direction of positive velocity in the boundary layer

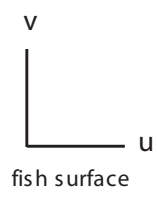

Figure 2-11: Velocity field (at phase $=0$ ) and boundary layer profiles for a cylinder oscillating parallel to a sculpin fish. Vectors of uniform length represent the direction of the flow, while colors indicate the velocity magnitude. Representative velocity profiles in the boundary layer are shown at phase $=0$ (see figure $2-13$ for phase $=\pi / 2$ plots). The solid lines are the predicted values using potential flow theory and Stokes' oscillatory flow next to flat surface. The data points are from the resolved boundary layer numerical simulations. The coarse grid solutions measure the velocity at mean height of $0.6 \mathrm{~mm}$ above the surface, which should be outside of an induced boundary layer for a cylinder oscillating at $50 \mathrm{~Hz}$. The cylinder is 3.7 diameters away from point on the fish surface directly beneath it. 
Instantaneous velocity field and boundary layer for a cylinder oscillating perpendicular to the fish

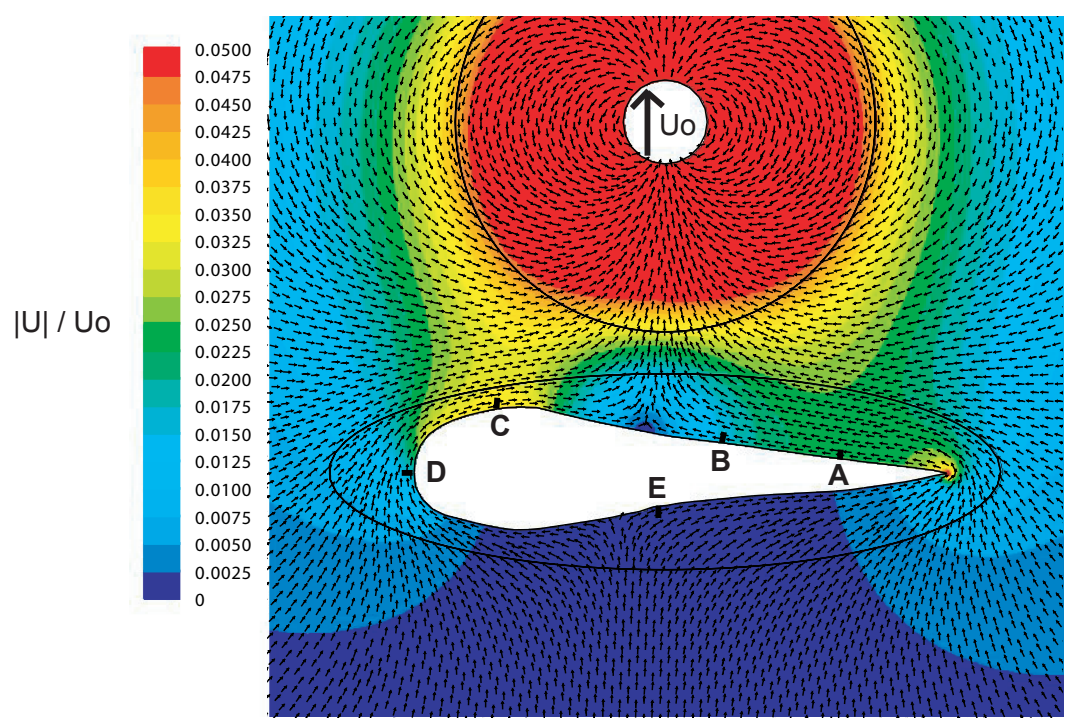

Phase $=0$
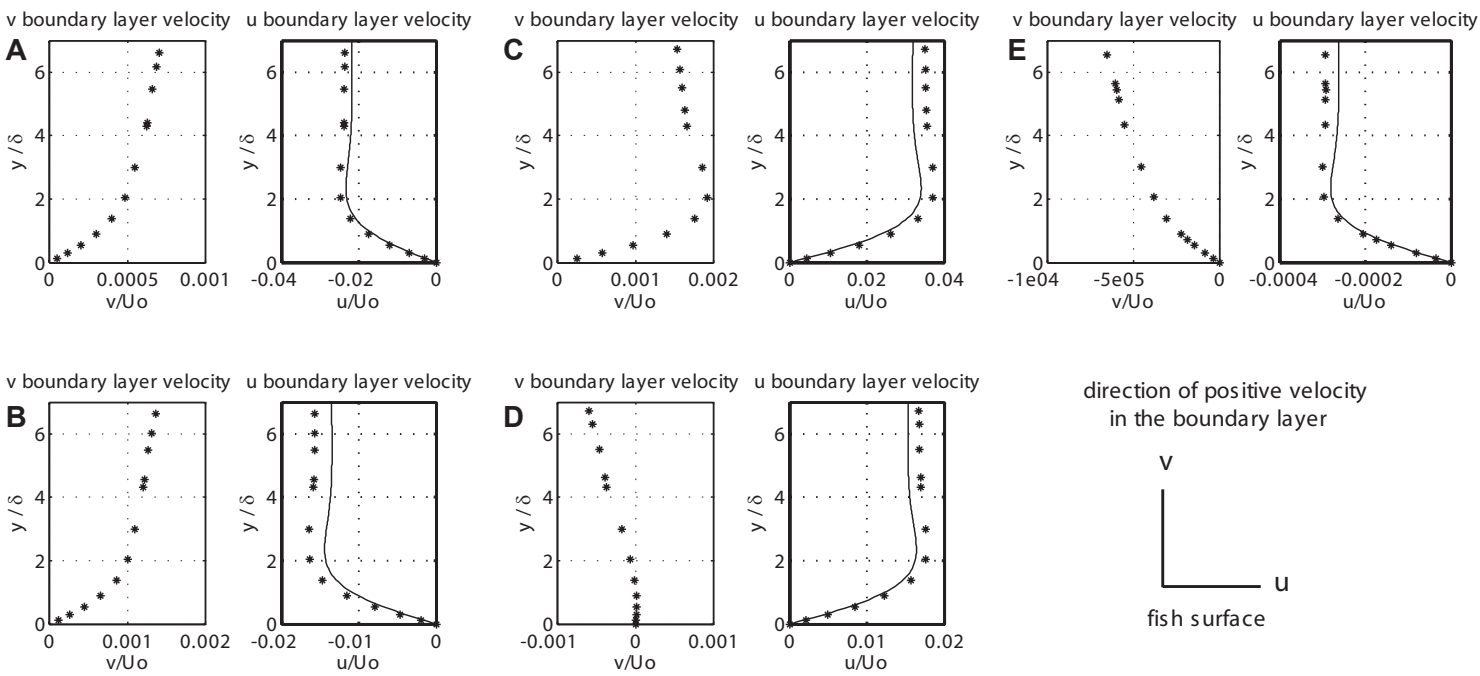

direction of positive velocity in the boundary layer

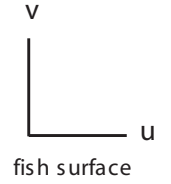

Figure 2-12: Velocity field (at phase $=0$ ) and boundary layer profiles for a cylinder oscillating perpendicular to a sculpin fish. The set up and plot explanation is the same as in figure 2-11. 


\section{Phase $=\pi / 2$}

Instantaneous velocity profile in the boundary layer for a cylinder oscillating parallel to the fish
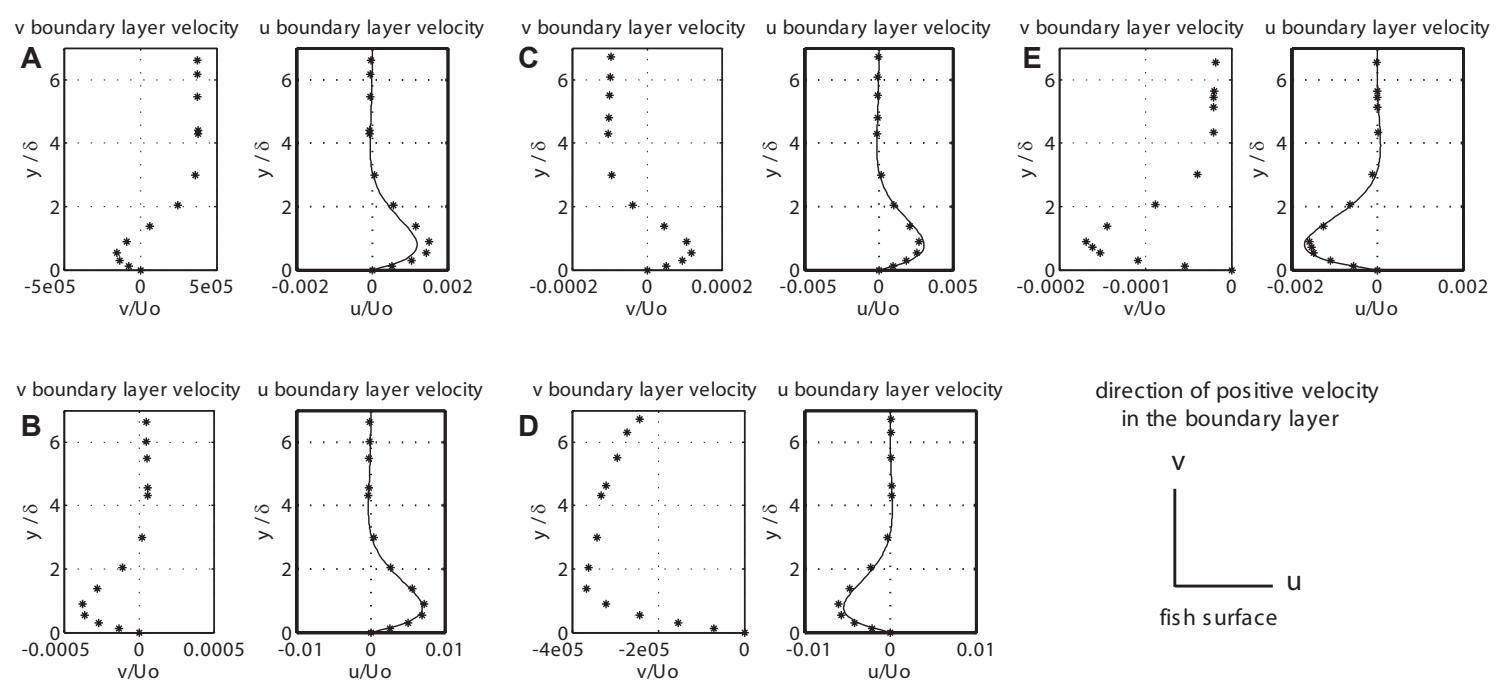

direction of positive velocity in the boundary layer

v

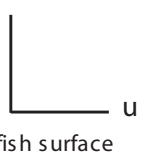

Instantaneous velocity profile in the boundary layer for a cylinder oscillating perpendicular to the fish
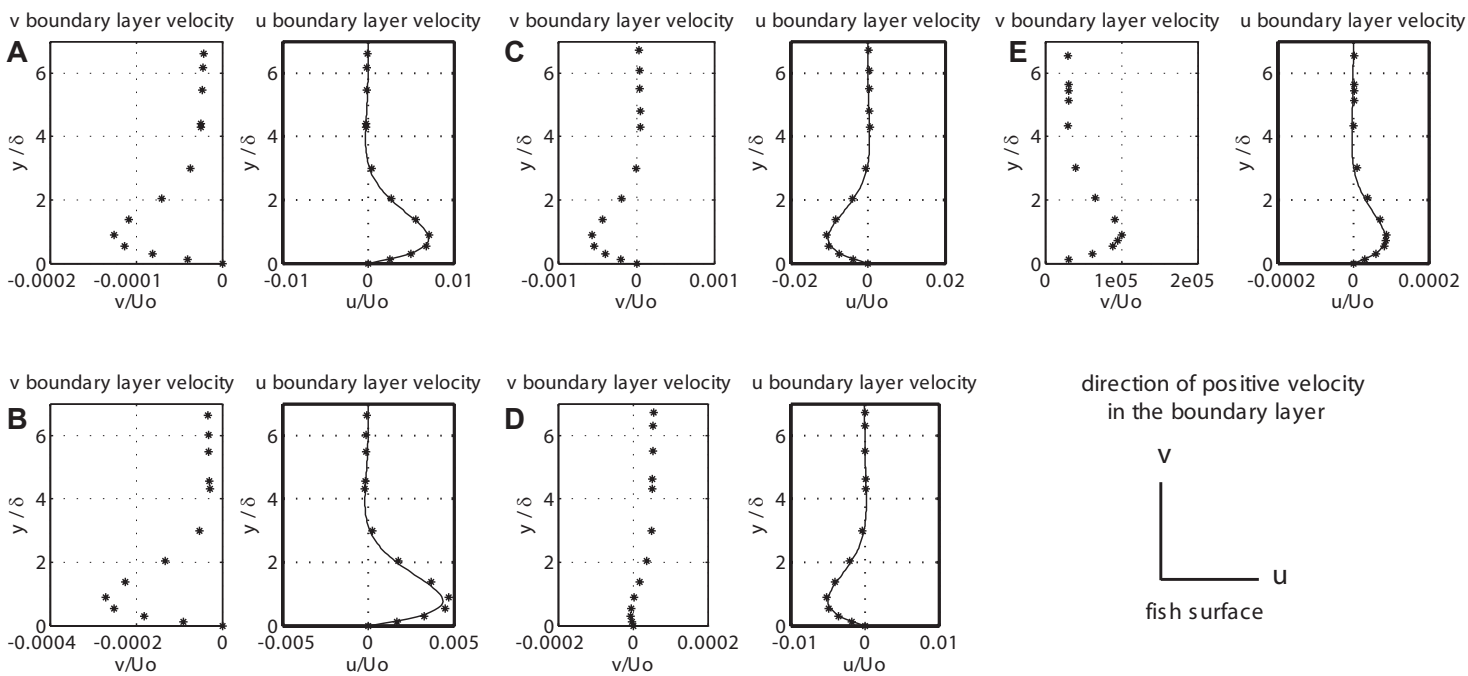

direction of positive velocity in the boundary layer
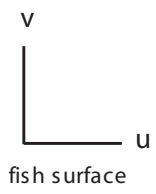

Figure 2-13: Instantaneous (phase $=\pi / 2$ ) velocity boundary layer profiles on a sculpin surface for a cylinder oscillating parallel and perpendicular to the longitudinal midline of a sculpin body. The points selected are those of figures 2-11 and 2-12. The solid lines are the predicted values using potential flow theory and Stokes' oscillatory flow next to flat surface. The data points are from the resolved boundary layer numerical simulations. The coarse grid solutions measure the velocity at mean height of 0.6 $\mathrm{mm}$ above the surface, which should be outside of an induced boundary layer for a cylinder oscillating at $50 \mathrm{~Hz}$. The cylinder is 3.7 diameters away from point on the fish surface directly beneath it. 


\section{Instantaneous velocity field and boundary layer for a cylinder oscillating parallel to the fish}

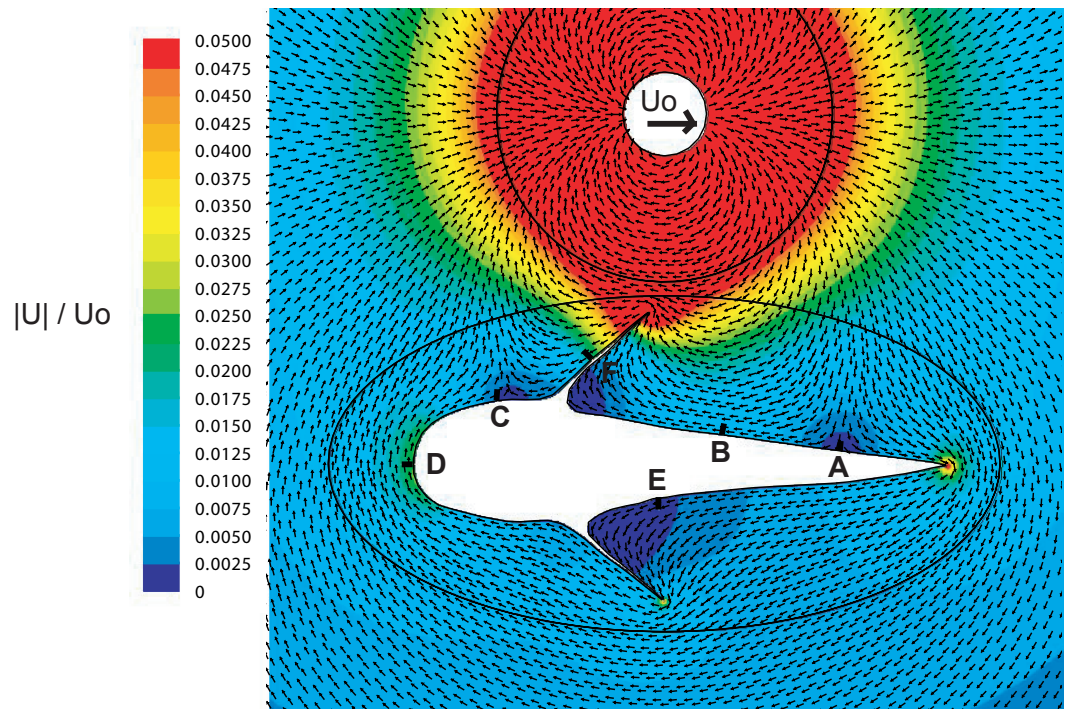

Phase $=0$
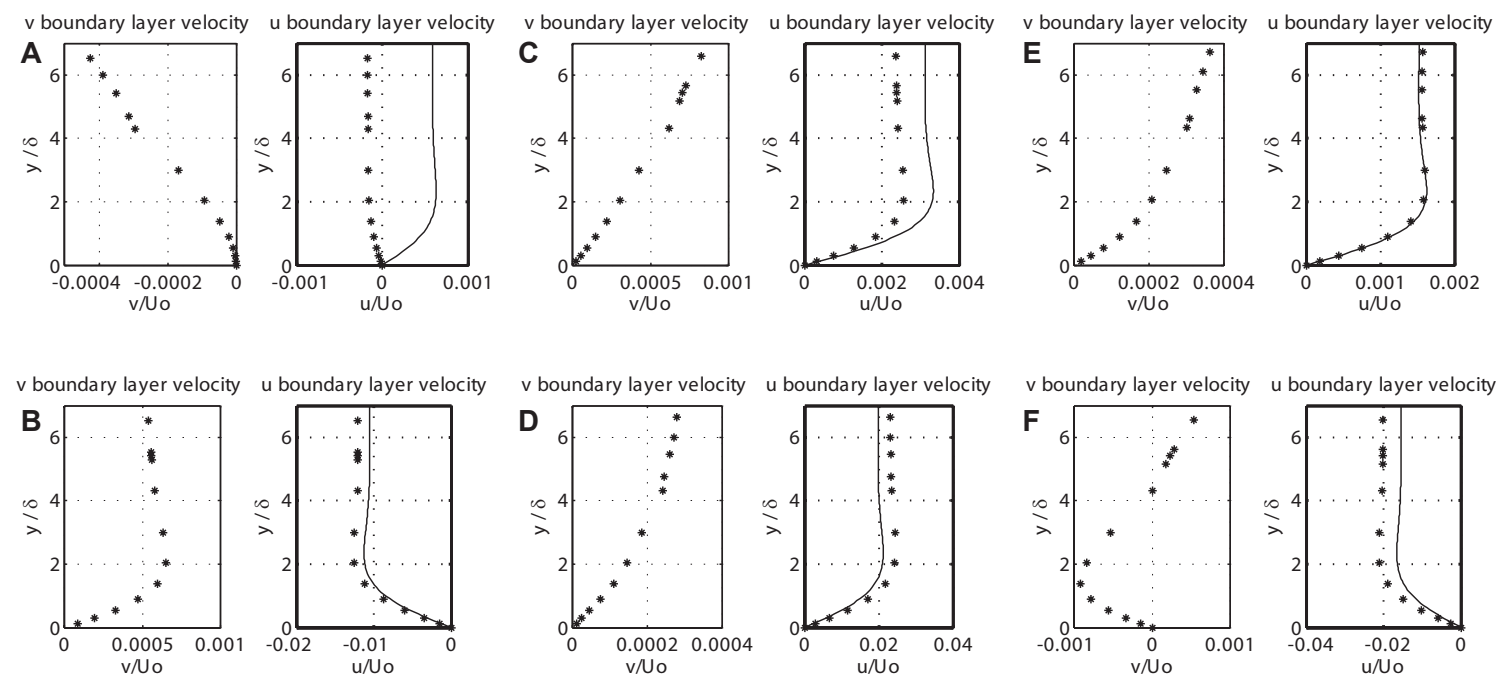

Figure 2-14: Velocity field (at phase $=0$ ) and boundary layer profiles for a cylinder oscillating parallel to a sculpin fish with pectoral fins extended. Vectors of uniform length represent the direction of the flow, while colors indicate the velocity magnitude. Representative velocity profiles in the boundary layer are shown at phase $=0$ (see figure $2-13$ for phase $=\pi / 2$ plots). The solid lines are the predicted values using potential flow theory and Stokes' oscillatory flow next to flat surface. The data points are from the resolved boundary layer numerical simulations. The coarse grid solutions measure the velocity at mean height of $0.6 \mathrm{~mm}$ above the surface, which should be outside of an induced boundary layer for a cylinder oscillating at $50 \mathrm{~Hz}$. The cylinder is 3.7 diameters away from point on the fish surface directly beneath it. 
Instantaneous velocity field and boundary layer for a cylinder oscillating perpendicular to the fish

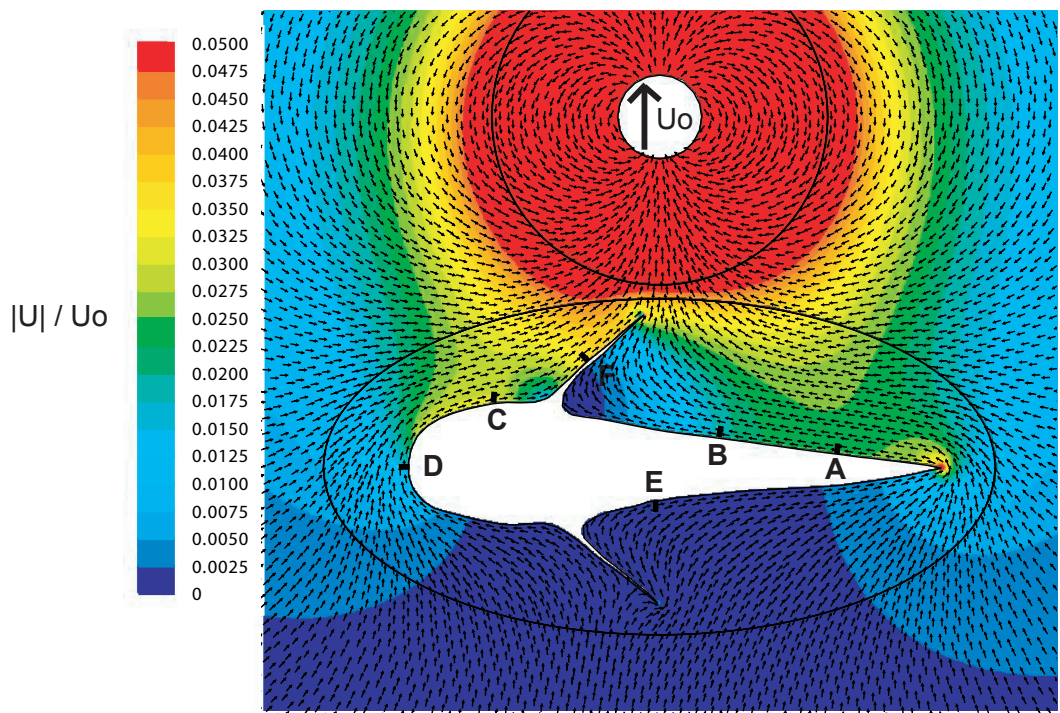

Phase $=0$
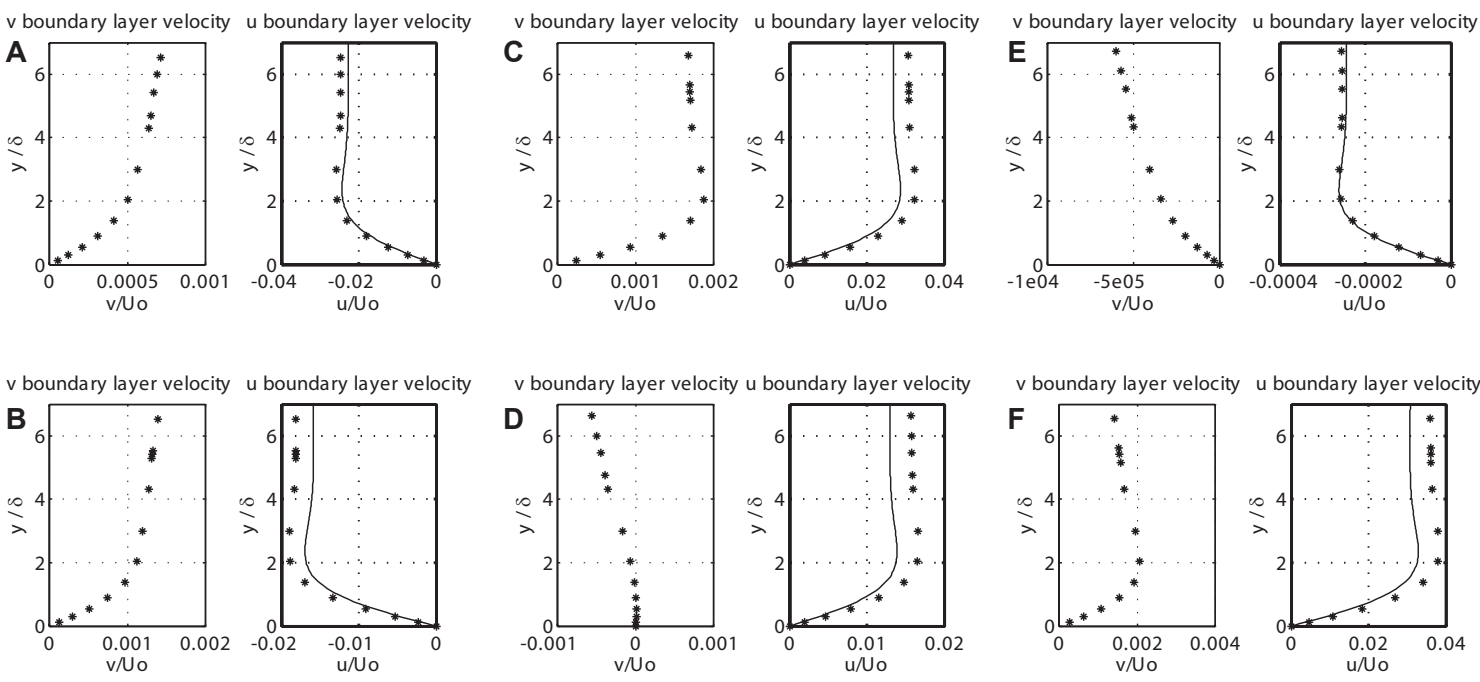

Figure 2-15: Velocity field (at phase $=0$ ) and boundary layer profiles for a cylinder oscillating perpendicular to a sculpin fish with fins extended. The set up and plot explanation is the same as in figure 2-14. 


\section{Phase $=\pi / 2$}

Instantaneous velocity profile in the boundary layer for a cylinder oscillating parallel to the fish
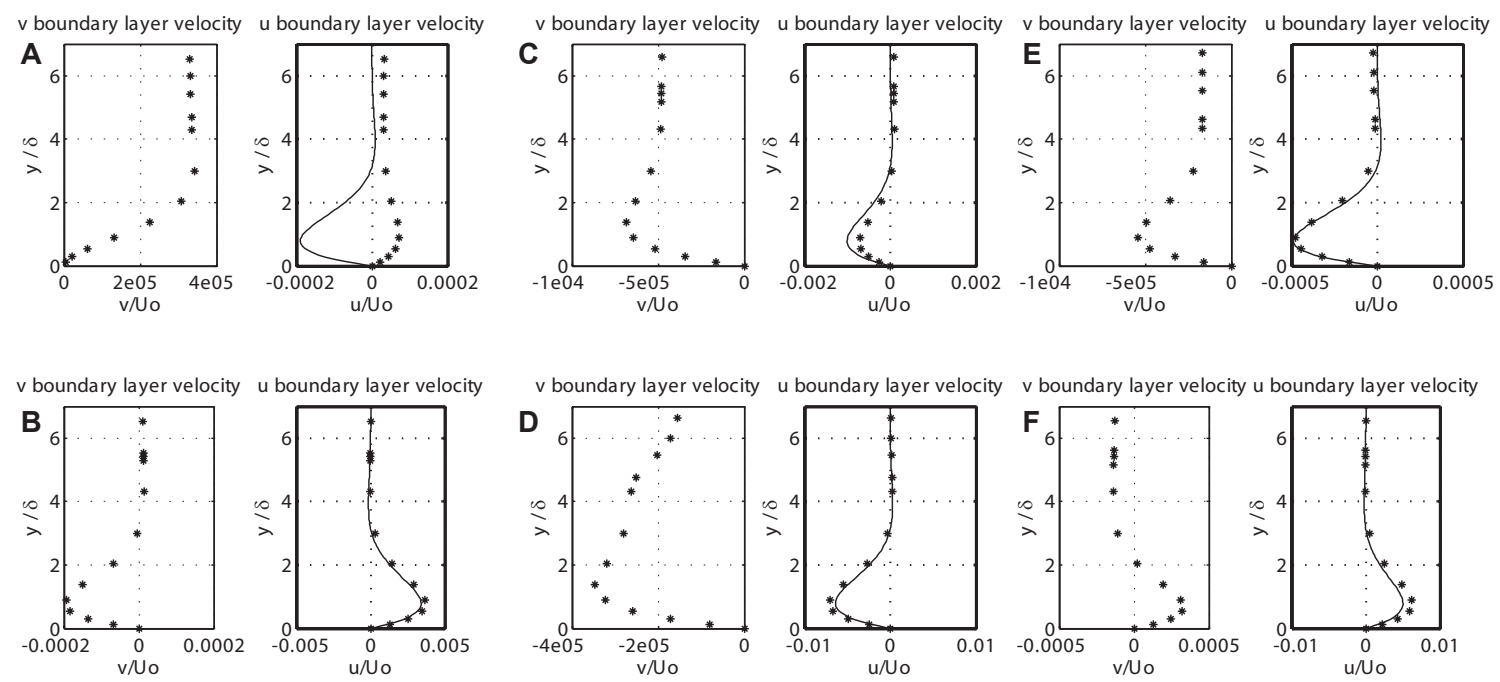

Instantaneous velocity profile in the boundary layer for a cylinder oscillating perpendicular to the fish
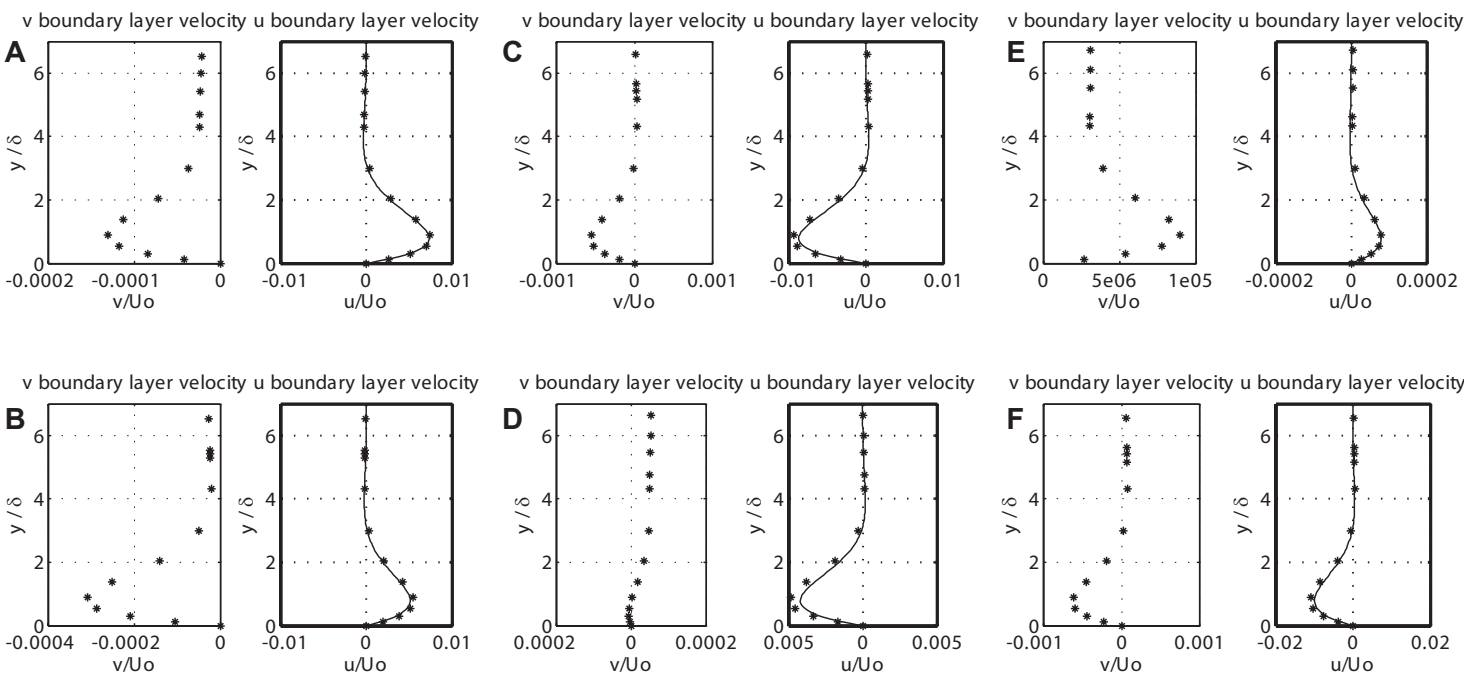

Figure 2-16: Instantaneous (phase $=\pi / 2$ ) velocity boundary layer profiles on a sculpin surface for a cylinder oscillating parallel and perpendicular to the longitudinal midline of a sculpin body. The points selected are those of figures 2-14 and 2-15. The solid lines are the predicted values using potential flow theory and Stokes' oscillatory flow next to flat surface. The data points are from the resolved boundary layer numerical simulations. The coarse grid solutions measure the velocity at mean height of 0.6 $\mathrm{mm}$ above the surface, which should be outside of an induced boundary layer for a cylinder oscillating at $50 \mathrm{~Hz}$. The cylinder is 3.7 diameters away from point on the fish surface directly beneath it. 
with pectoral fins extended at phase $=0$ when the cylinder oscillates parallel to the longitudinal midline of the fish. Where there was an increased region of flow near the body with fins retracted (see figure 2-11), the fins block the flow in that region and eliminate that effect. The velocity stagnation points have moved slightly closer to the center of the fish, and there are additional regions of reduced flow in the pockets formed between the pectoral fins and the side of the fish body. Again, within the boundary layer, $v$ is around an order of magnitude less than $u$ (at least), and predictions for $v$ would be in error (as compared to the flat wall case, figure 2-6). The predicted $u$ profile matches the actual results quite well at almost every location, except in the stagnation point where direction of flow is oppositely predicted. This is of little consequence, owing to the fact that there has to be an exact point that the flow changes directions, and the coarse mesh solution misses that exact point. The predictions for the velocity on either adjacent cell in the coarse grid are back on track (not shown). The same missed prediction shows up at point $\mathrm{A}$ in the phase $=\pi / 2$ plots, shown in figure 2-16 (top set of plots), and all the rest of the selected points show excellent agreement.

When the cylinder oscillates perpendicular to the side of the sculpin (figure 2-15), the body presence also alters the flow field in a similar fashion to the fins-retracted case (figure 2-12). This is expected, as the extended fins are largely in the direction of flow in this particular fish-prey configuration, and have little impact. On the opposite side of the fish, the stagnation point that was near point $\mathrm{E}$ has now located on the pectoral fin itself, and the fin insertion point acts as the new stagnation point to the fish. The shape of the $u$ velocity profiles within the boundary layer is well predicted at all points, though again the magnitudes are often slightly diminished. When phase $=\pi / 2$, as shown in figure 2-16 (bottom set of plots), the $u$ velocity profiles are very well predicted at all points.

Figure 2-17 shows how the presence of the sculpin body affects the pressure field around a dipole source. The concentrations of pressure changes appear in the same places as the regions of increased velocity magnitude (figures 2-11, 2-12, 2-14, and 2-15). When the cylinder oscillates perpendicular to the longitudinal midline of the 
fish, one side of the fish is shielded from the source almost entirely. This is likely an exaggerated 2D effect, since the body acts like a wall of infinite height and forces flow around rather than over it. However, when the prey is close enough, this may well represent the midline of the fish.

\subsubsection{Changing dipole locations}

The final goal of this chapter is to use a series of $2 \mathrm{D}$ simulations to look at what effect extended pectoral fins have on the received dipole signal. The signal to be considered is the pressure gradient, which stimulates the canal lateral line. Figure 2-4 shows the basic pressure gradient patterns that appear on a flat surface when a cylinder oscillates in a range from parallel to perpendicular relative to the wall. These same patterns appear on the fish body, though differences should exist because the body is of finite size in relation to the dipole source, because it has various grades of curvature, and because it can have extended body parts. Figures 2-18 and 2-19 show what happens when the dipole source is placed at various distances, locations, and orientations relative to the fish body. The patterns shown are of instantaneous pressure (phase $=\pi / 2$ ), similar to figure $2-17$. The direction of pressure difference is defined in figure $2-4$.

For the sculpin with fins retracted, figure 2-18 shows that the same pressure gradient patterns that would appear on a flat wall are evident over the body of the fish. When the cylinder oscillates at an angle of 0 degrees relative to the longitudinal body axis, the location of the peak matches the point where a line drawn from the cylinder to the side of the fish is the perpendicular bisector of the side of the fish, at the point on the side of the fish which is closest to the cylinder. This is the exact same location for the central zero crossing that occurs when the cylinder oscillates perpendicular to the side of the fish. When the cylinder oscillates at other orientations (such as 30 and 60 degrees) the pressure gradient pattern is a weighted combination of the two main patterns (Curcic-Blake and van Netten, 2005).

These patterns stay evident as the location of the cylinder is moved from the front of the fish, to the side, and finally to the tail. If the dipole source where to 


\section{Instantaneous pressure contours (phase $=\pi / 2$ ) for an oscillating cylinder nearby a sculpin with fins retracted/extended}

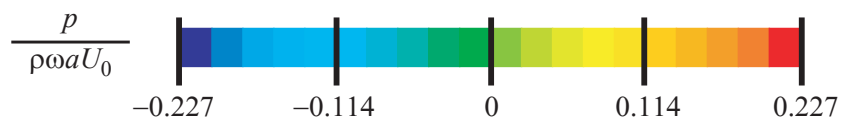

Oscillating parallel
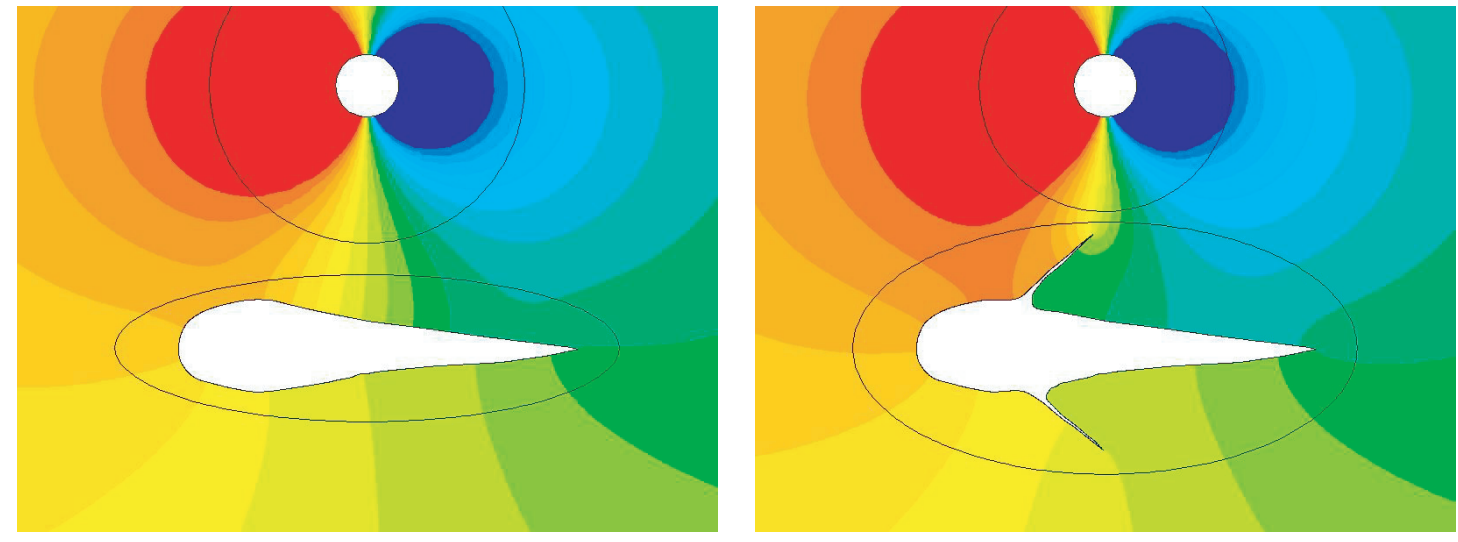

Oscillating perpendicular
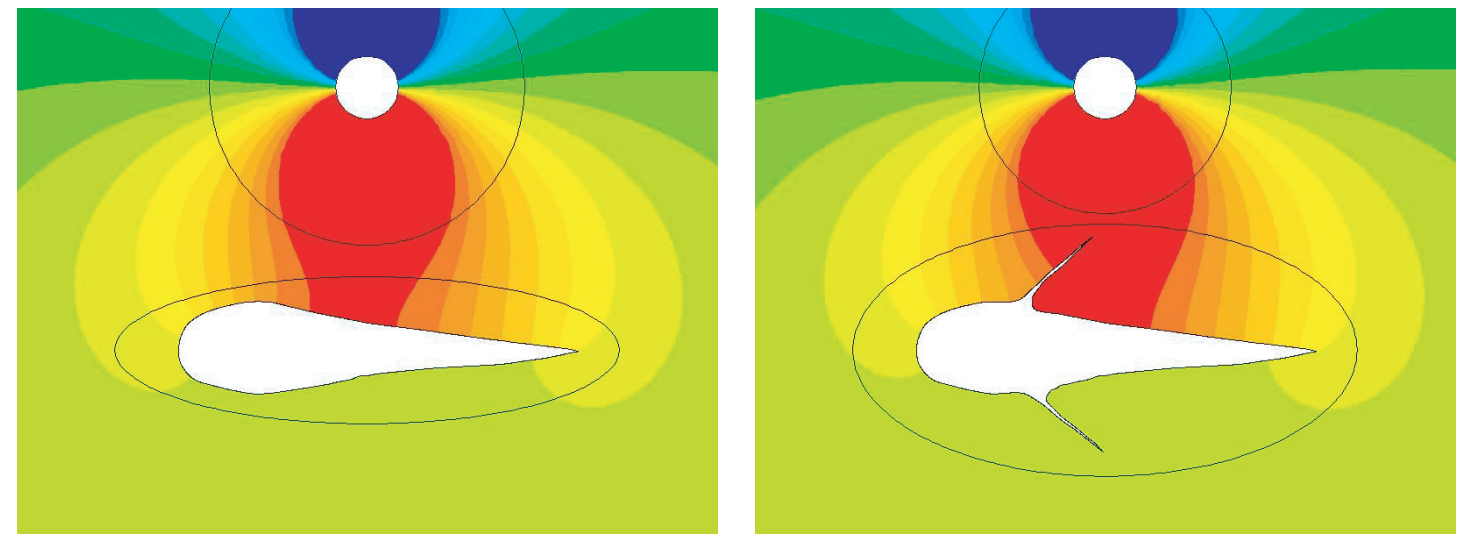

Figure 2-17: Instantaneous pressure fields (phase $=\pi / 2$ around sculpin bodies near a dipole source. The directions of cylinder oscillation are parallel and perpendicular to the longitudinal midline of the body. The cylinder is located 3.7 diameters from the point on side of the fish directly beneath it. 
maintain a steady amplitude oscillation, then the magnitude of the peaks would be an additional indicator of the source distance. As the cylinder nears the tail, the information becomes more ambiguous, since the pressure gradient pattern is only partially shown on the fish. The clearest information available are the zero crossings, or stagnation points, of the parallel oscillating cylinder, since this unambiguously encodes source distance information.

The presence of the fins alters the pressure difference trends by forcing a zero point at the fin insertion points on the canal lateral lines. This is most significant when the dipole source is near the nose of the fish, because the patterns are only partially displayed. When the cylinder is near the side of the fish, the pressure gradient patterns are present in their full form, and the fins have less of an effect.

Figure 2-19 shows the pressure gradient patterns on the side of the fish body when the dipole source is moved a little less than one body length away. The most obvious difference is that the magnitude is much reduced relative to a constant amplitude stimulation. Note that the scale in the plots has been reduced in order to better show the patterns. Secondly, the patterns are less defined and more spread out, which allows for the fins to have a more significant effect since the pressure gradient patterns are more likely to cross over the pectoral fins. What also becomes evident is that near the tail, the pressure gradient pattern wraps around the fish body. Figure 2-20 shows how the signal shape is effectively preserved as it wraps around the body. 


\section{Dipole pressure gradient patterns due to a cylinder oscillating between 0-90 degrees}

Sculpin with fins retracted
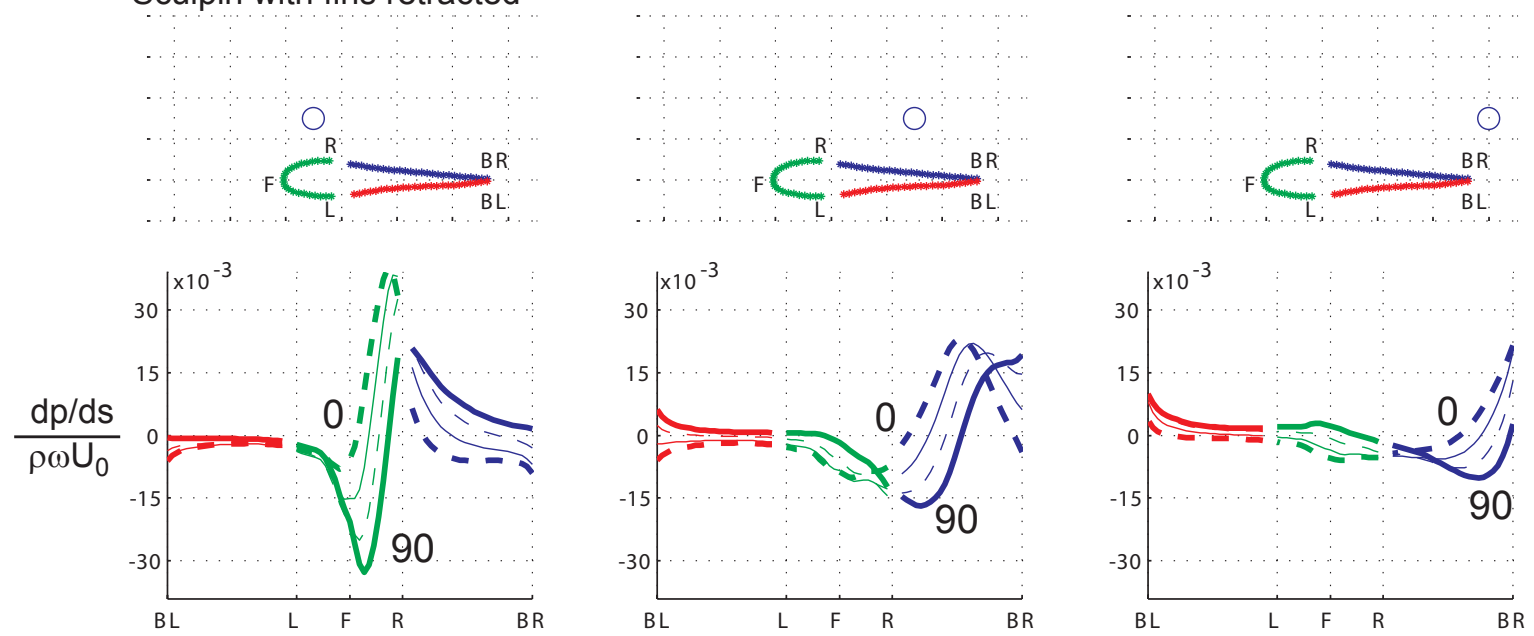

Sculpin with fins extended
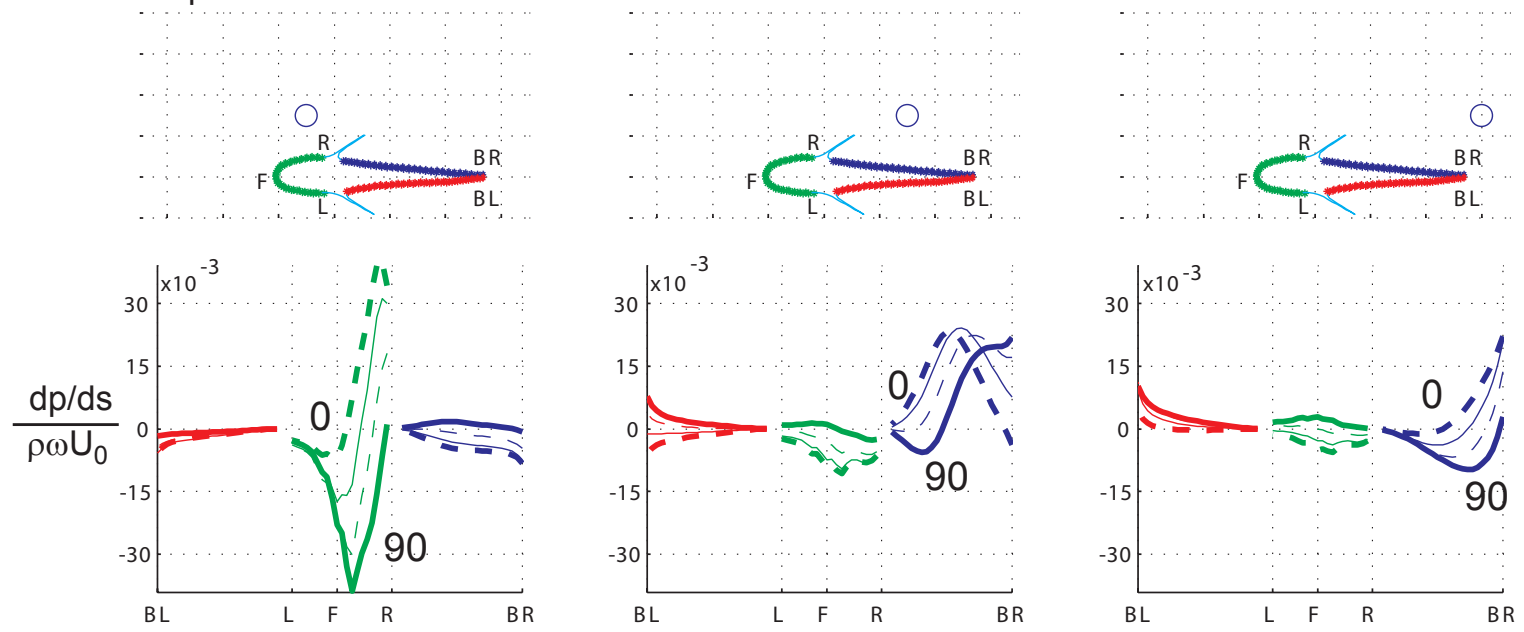

Figure 2-18: Dipole pressure gradient patterns on a sculpin in close proximity to an oscillating cylinder. The cylinder oscillates between 0 and 90 degrees relative to the longitudinal axis of the fish. Node points on the surface of the fish represent pore openings to the canal lateral line, and are spaced $2 \mathrm{~mm}$ apart. The lateral line is divided into a source side trunk lateral line (blue), front lateral line (green), and back side trunk lateral line (red). The plots represent the pressure difference along the lateral line, as defined in figure 2-4. The pressure gradient patterns occur at phase $=\pi / 2$, similar to what is seen in figure 2-17. 


\section{Dipole pressure gradient patterns due to a cylinder oscillating between $\mathbf{0 - 9 0}$ degrees}

Sculpin with fins retracted
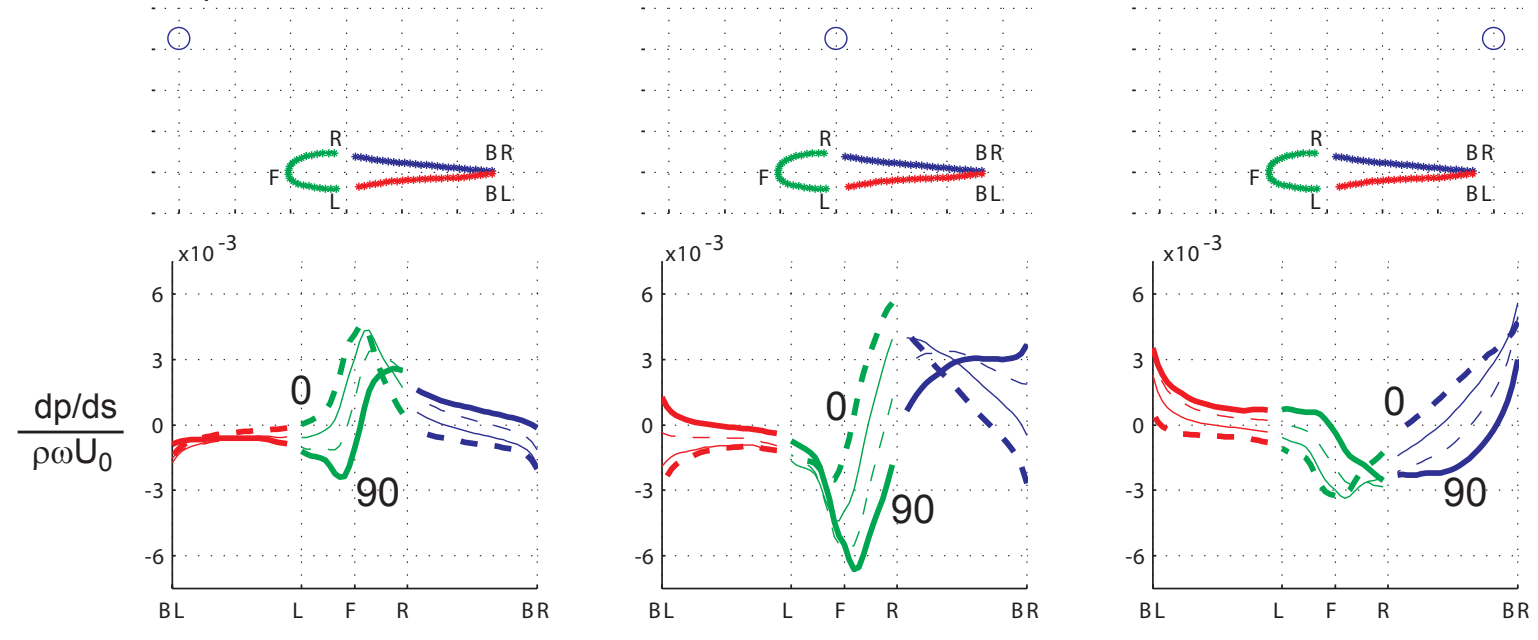

Sculpin with fins extended
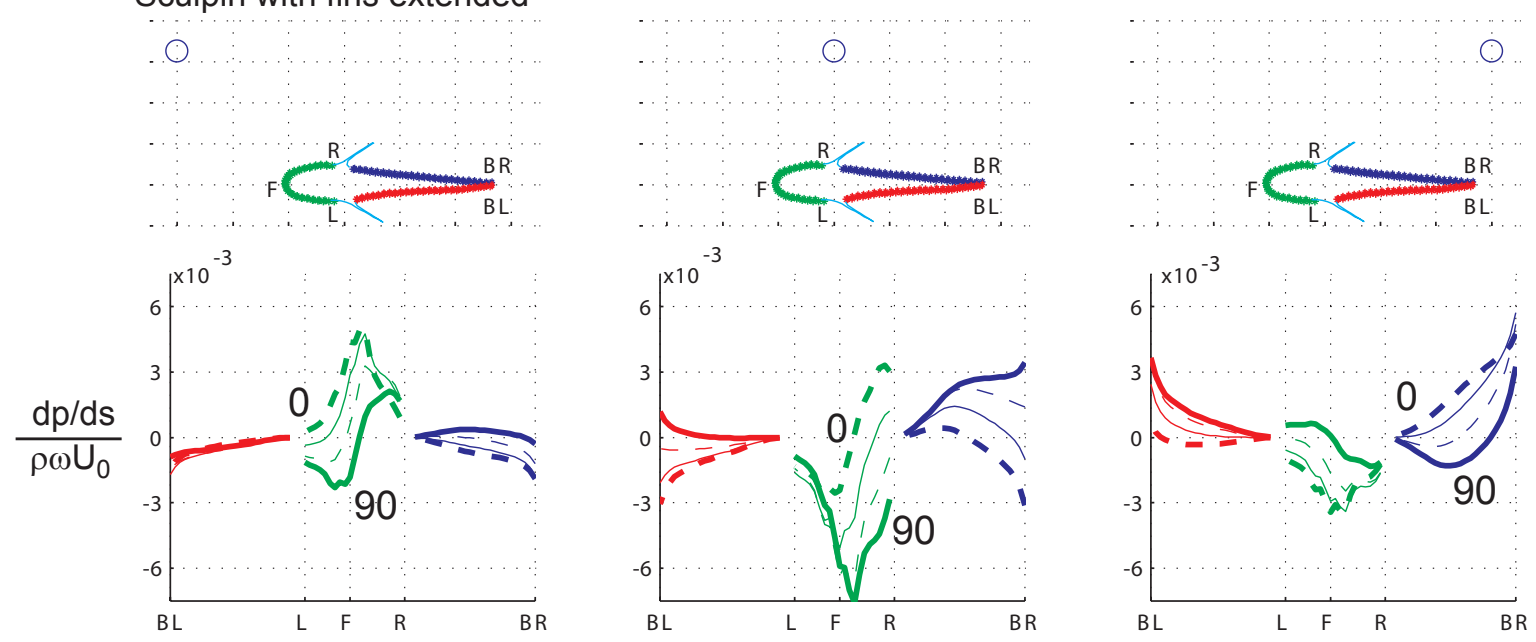

Figure 2-19: Dipole pressure gradient patterns on a sculpin a little less than a body length away from an oscillating cylinder. The set up and explanation are given in figure 2-18. 


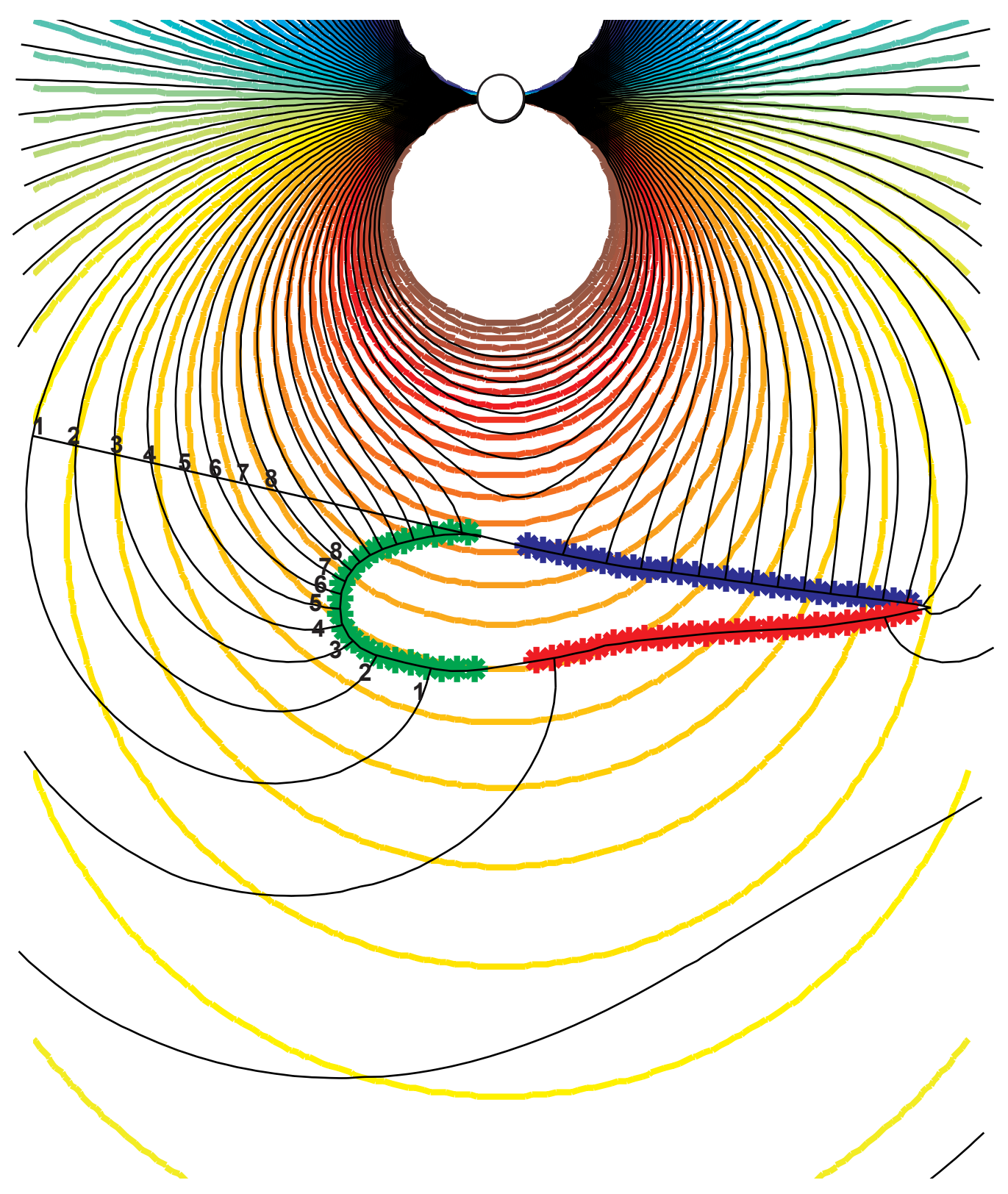

Figure 2-20: Use of the entire lateral line for source localization. Instantaneous (phase $=\pi / 2$ ) iso-pressure contour lines are drawn for a cylinder oscillating alone in an unbounded medium (colored lines), and next to a sculpin body (black lines). The presence of the body causes the pressure to concentrate around curved surfaces and points, effectively extending its lateral line surface and 'feeling' more of the dipole source. This can explain why the pressure gradient patterns of figures 2-18 and 2-19 capture much of the patterns seen along a flat wall (figure 2-4). 


\subsection{Discussion}

\section{Validation of the course grid numerical simulations}

A primary goal of this chapter was to see if the coarse grid (unresolved boundary layer) simulations could accurately predict the velocity and pressure outside the oscillating boundary layer around a wall, and around various shaped fish bodies. The purpose of this exercise was to lay the foundation for doing 3D coarse grid simulations, where the computational restrictions prevent resolving the boundary layer flow around the sculpin for time dependent flows. The signal to the canal lateral line is the pressure gradient, and since canal pores are spaced approximately $2 \mathrm{~mm}$ apart, a coarse grid resolution is able to provide this signal in its entirety. It was important to verify that the dipole stimulus signal in still water is presented without distortion.

Figures 2-5, 2-8, 2-9, and 2-10 confirmed that the boundary layer around a wall and around a fish body does not affect the pressure signal to the surface. Even when there were body parts, such as extended pectoral fins, the coarse grid numerical simulations gave the same pressure results as the resolved boundary layer simulations. This gives confidence that the pressure signals, created by the dipole stimulus, will be correctly predicted in all of the simulations performed.

As for predicting the velocity outside of the boundary layer, overall the results were excellent, but not identical. The most noticeable deviations of and differences between the coarse grid mesh predictions and the resolved boundary layer results were at locations of peak velocity magnitude, where the values were less than actual. Where there were sharp changes in velocity over short distances, such as near the tail tip, the coarse grid mesh is not able to represent those aspects of flow, since it averages the flow locally by nature of its individual cell sizes. Nonetheless, over much of the region of the fish, the velocity is well predicted.

There is one study that shows deviation of the predicted velocity outside the boundary layer using numerical simulations. Goulet et al. (2008) performed a 2D simulation of a sphere next to a wall in order to see if potential flow theory would match numerical results. Their results showed unphysical deviations of velocity, which 
they attribute to viscosity effects near the wall. Based on the results of this thesis, it is unlikely that their numerical results were correct. Details of the numerical simulation were omitted, so it is unclear how they arrived at their results. They conclude that the boundary layer effects can be neglected, which is also incorrect. However, their work is really based on the location of the stagnation points of velocity and pressure gradient along the lateral line of fish, which is well predicted by the use potential flow theory.

It is difficult to generalize results of predicting the velocity in still water conditions to that of moving water conditions, because it is not clear at this point if the interactions of the flow, stimulus signal, and boundary layer interact in a linear fashion. If not, then the coarse grid mesh will likely be limited to representing the pressure signals when there is more complex flow interaction.

\section{Validation of predicting the boundary layer flow along a surface due to an oscillatory dipole source}

It is a little bit surprising how well the boundary layer flow could be predicted by finding the velocity outside the boundary layer (using either potential flow theory or a coarse grid simulation), and applying Stokes' solution for flow in an oscillatory boundary layer next to a flat plate. The main surprise is that although a dipole source creates an uneven pressure gradient over the length of the wall (see figure 2-4), it does very well in predicting the boundary layer flow (see figures 2-6, 2-7, and 2-11-2-16) that is based upon a spatially uniform pressure gradient.

The errors that did occur were not in predicting the shape of the $u$ profile, but in predicting the magnitude. When the surface parallel velocity outside the boundary layer, $U_{\infty}$, was wrongly predicted, then the associated scaling error propagated through the rest of the boundary layer profile (see equation 2.1). These errors often occurred in locations where the coarse grid solution averaged the changes that were occurring in velocity, such as in and around the stagnation points, rather than resolving the changes. 
Predicting the surface normal velocity component, $v$, really only worked well in the case of a flat surface (see figures 2-6 and 2-7). With a curved surface of finite dimension, the flow field around the fish from the dipole stimulus is much less uniform, and consequently the flow is being forced into or away from the sides of a fish more often. This results in greater $v$ magnitudes, and the flow itself is more complex than would be predicted by potential flow theory. These deviations actually represent the error to the $u$ velocity profile predictions, and in general, are an order of magnitude less than the $u$ velocities.

\section{The effect of body parts on dipole source localization}

A dipole source will produce a signature spatial gradient (see figure 2-4) along the lateral line, providing information about the location of the source relative to the body. Figures 2-18 and 2-18 show that these basic signals are well preserved on curved fish bodies, except in the tail region.

Studies that compared the neural responses along the lateral line with modeled dipole pressure gradients showed that the excitation patterns were encoded (Coombs et al, 1996). This includes the accurate encoding of pressure gradient zero crossing for a dipole source at various distances from the lateral line (Curcic-Blake and van Netten, 2006). Behavioral studies confirm that the lateral line canal system, and their encoded excitation patterns, plays an important role in prey (dipole source) localization (Coombs and Conley, 1997a,1997b). Of launched attacks with the source at $3 \mathrm{~cm}$ away, $75 \%$ of them occurred with the source more laterally oriented to the fish body axis, where there would be better encoding of the zero crossings (Coombs and Conley, 1997b).

The extension of pectoral fins has the effect of 'pinching' off the signal at the point of fin insertion on the side of the fish, because in that region the flow velocity has been reduced. The interaction of the fins with the dipole flow field also affects other regions in close proximity. When the fins block the flow to any degree, the pressure is increased on one side and decreased on the other. Secondly, the concentration of isopressure lines (see figure 2-17) changes, so that there is an increase in the pressure 
gradient on the side that blocks the flow, and a decreased pressure gradient on the side blocked from the flow. These affects are accentuated by the nature of $2 \mathrm{D}$ bodies, and are expected to be less around 3D bodies were the flow has a third dimension to move in and around the finite body.

The fins also have the ability to distort the received dipole signal, especially when either a peak or stagnation point is interrupted by the presence of the fin. Likewise, the finite size of the fish limits signal reception by cutting short a signal for a source that is too far away. This has been pointed out by Curcic-Blake and van Netten (2006), who determined the shortest and farthest distances an oscillating dipole sphere can be from the side of a fish and still manage to contain the peaks and stagnation points in the signal to one side of the lateral line.

Evidence suggests that information processing by the fish is unilateral (Coombs et al, 2000). The approach strategies of the Mottled Sculpin show that it approaches in steps, orienting one side at a time. In experiments where fish had one side denervated, there was no effect in its ability to detect and attack prey on its good side. Finally, poor frontal performance suggests that spatial information is not integrated from side to side (Coombs et al, 2000).

Though dipole localization results were presented for the canal lateral line system, the velocity at the edge of the boundary layer has similar peaks and stagnation point locations because it also is the gradient of the potential field (compare equation 2.14 with 2.12 and 2.13). Goulet et al. (2008) confirm this, and show that both velocity and pressure gradient signals yield the same information (though scaled differently). Therefore, the superficial neuromasts could in theory have a significant role in dipole localization, since they can more encode 3D information by virtue of the hair orientations around the body.

It has been argued that the oscillating dipole stimulus is an unbiological signal since actual prey and fish motions lead to more complex signals. For example, the flapping of the pectoral fin for motion, or the feeding currents by smaller prey, will produce the relevant hydrodynamic signals that are not of a dipole nature. In general, the flow produced will be species specific (Jansen, 2004). With this in mind, it must 
be remembered that the results presented in this chapter are only of basic value, upon which further investigations can build. 


\section{Chapter 3}

\section{Thresholds of detection and saturation in still water}

In chapter 1, the basic problems to be addressed in this thesis were presented - namely the signal-body interaction and the flow-signal-body interaction. These are the hydrodynamic interactions that this thesis can begin to address. However, in order to understand how these signals impact the fish, or to understand how the fish are able to extract the relevant signals and reject others, the mechanisms of the lateral line system which encode and filter the signals must be addressed. In particular, one desired result is an input-output function for the lateral line neuromast motions, so that different scenarios can be assessed for their impact on the neuromast detection and saturation thresholds. That requires a determination of the micromechanical properties of the neuromasts, and the micro-fluidic properties of their local hydrodynamic environments.

Fortunately, there has been a substantial amount of research on the lateral line of fish and amphibians over the last number of decades, and great strides have been made in the understanding of its structure and function. The first part of this chapter will summarize the basic morphology of the fish lateral line, and compare some of the analytical models available which predict how the canal and superficial neuromasts will interact with and respond to their local hydrodynamic environments. These models capture the filtering properties of the neuromasts themselves, coupled with 
the filtering characteristics of the surface boundary layer and of the subdermal canal channels that they reside in. These models have been verified experimentally for certain fish, but are clearly meant to be applicable to a large number of species of fish. This thesis has chosen the Mottled Sculpin for illustrative purposes, and will discuss how these models might apply.

Beyond the mechanical aspects of signal encoding and filtering, there is also the likelihood that the fish further process the signals in their brain. There are both afferent nerves from the lateral line, sending signals to the brain, and efferent nerves to the lateral line, receiving signals from the brain. Feedback loops can allow for complex possibilities of signal processing. For instance, local hair cells are able to adapt to signals by lessoning their sensitivity to the hair cell stimulus (Popper and Fay, 1999; Eatock, 2000). This means that what the fish are able to detect, either at some minimal threshold, or at some maximal threshold, may be dependent on what the fish 'decides' to listen for.

What is most important to the fish is encoding enough relevant information from the local hydrodynamic environment in order to make decisions about what is near by, and where it might be located relative to itself. Namely, does it need to stay swimming in a school of fish, avoid some obstacle, avoid some predator, track some prey, communicate with a potential mate, etc.? In each of these situations, there is some minimal amount of information that uniquely distinguishes itself from some other situation, and the fish must (and do) have the requisite equipment to accurately encode and process this information. The source information will have amplitude, temporal/spectral, and spatial content.

This thesis will be able to simulate the hydrodynamics of some typical flow environments and of some typical prey signals. The temporal/spectral and spatial content can be examined to see what is required in order to extract the desirable signals. This chapter serves to further this discussion by hypothesizing how the signal might realistically be encoded by the mechanical and physical aspects of the lateral line system for the Mottled Sculpin. Secondly, there is some evidence that there are limits to hair cell signal adaptation, in particular to types of a.c. noise that are present in 
background flows (Chaugnaud et al., 2006). This thesis can begin to address what types of flow environments lead to sources of a.c. noise, and what impact this might have on detecting desirable signals.

The goal of this chapter is to present the basic morphology of the fish lateral line, and to compare some of the prominent analytical models which predict how the canal and superficial neuromasts interact with and respond to their local hydrodynamic environments. These models are then adapted specifically for the Mottled Sculpin. The mechanical filtering properties of the lateral line system, as modeled in this chapter, will form the basis for assessing the effects of background flow environments on signal recovery in chapter 5 .

\subsection{The lateral line system of fish}

Fish have a number of systems for detecting vibrations in the water - the inner ear, the swim bladder (which also stimulates the inner ear), and the lateral line system. Each system ultimately causes the bending of local ciliary bundles, which in turn causes the supporting hair cell to fire synaptic nerve signals. The ciliary bundles have short individual hairs called stereocilia, which progress in size up to the tallest hair, the kinocilium (Flock, 1967). The ciliary bundles are oriented to be excited when bent in one direction (toward the kinocilium), and inhibited in when bent in the opposite direction (see figure 3-1). Hair cells are often oriented with their ciliary bundles facing opposite each other, so that bidirectional motions are encoded (Flock and Wersall, 1962). This is clearly an important feature for assessing the frequency content of signals.

In the inner ear system, the ciliary bundles have solid masses called otoliths above them, and others have gelatinous coverings. In both cases, as the fish moves, these masses lag behind due to their inertia, and in turn, cause the touching ciliary bundles to bend with them (Popper and Fay, 1999). Thus, the inner ear acts as an inertial guidance system, which detects the whole body accelerations of the fish. These accelerations can be caused by self movement, or by an external gravity or pressure 


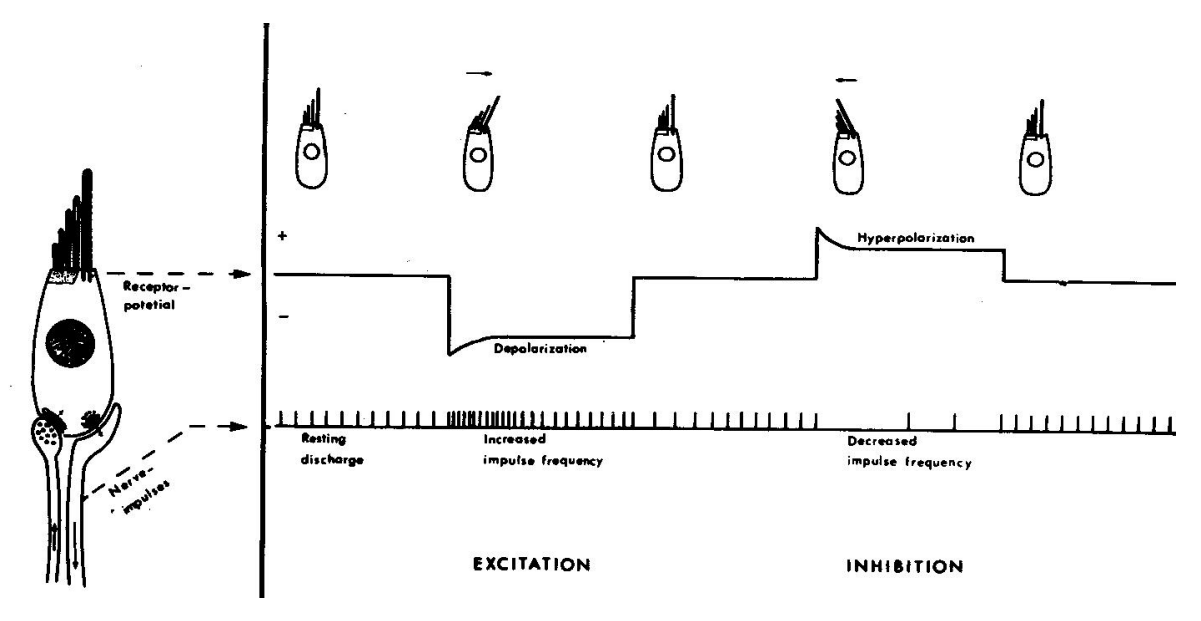

Figure 3-1: Diagrammatic illustration of the proposed theory of hair-cell function, showing the relation between receptor potential and nerve impulse frequency when the sensory hairs are inclined toward or away from the kinocilium. Diagram and caption from Flock (1967), Figure. 14., found in Cahn, Phyllis. Lateral Line Detectors. p. 182. Reprinted with permission of Indiana University Press.

field. Traveling sound waves would cause the body of the fish to vibrate, alerting the fish of some proximate disturbance. By virtue of responding to whole body motions, it also means that the fish essentially integrates all spatial hydrodynamic information over its body, thereby making it a poor detector of the spatial resolution of the hydrodynamic field (Braun and Coombs, 2000).

For some fishes, its swim bladder can also act as a sound source and sound receiver. As the density inside the swim bladder is much less than that of its body or the surrounding water, incident sound waves can cause the bladder to reradiate the energy, providing a stimulus for any otolith organs in close proximity (Popper and Fay, 1999), and occasionally the lateral line (Coombs and Montgomery, 1999). The motions caused by the oscillating air cavity might stimulate different motions in the inner ear than would otherwise be caused by the whole body motions, thus providing additional information for sound source identification and localization.

The lateral line has a number of neuromasts, or hair cells groups, located on the surface of the fish and in subdermal canal systems. These hair cell groups are covered by a protective, gelatinous cupula. As water moves past the cupula, viscous drag forces cause shearing motions, and the hairs inside bend with the cupula. The lateral 
line system can often be recognized as a darkened thin line that proceeds from the front of the fish towards its tail. Extensive information on the lateral line system can be found in reviews by Bleckmann (1993), Coombs and Montgomery (1999), and van Netten (2006). The most important feature of the lateral line, as compared to the inner ear or swim bladder, is that it provides much more spatial information about the hydrodynamic field by virtue of their arrangements over the whole body.

Since the actual placement and branching of the lateral line is species specific, it is useful to select a particular species to get the general idea of the lateral line morphology. The lateral line system of the Mottled Sculpin, as presented in the work of Janssen et al. (1987), is shown in figure 3-2. The drawing shows the arrangement and orientation of both canal and superficial neuromasts. Of particular importance is that the superficial neuromasts are often situated orthogonally to each other. Along the trunk lateral line these pairings are side by side, while at one end of the mandibular canal there are two orthogonal arrays. This is significant because it allows for the resolution of the two-dimensional vector flow field tangential to its skin surface. Canal neuromasts are oriented to respond to water motions in alignment with each local branch of the canal they are situated in. Only one component of the flow field can be resolved.

\subsubsection{Canal neuromasts}

The canal neuromasts are located in a channel just under the surface of the skin with open pores that allow the entrance of water. Each neuromast contains many ciliary bundles (see figure 3-3). Janssen et al. (1987) found that the canal neuromasts of the Mottled Sculpin have its sensory strip of hair cells surrounded by mantle cells, so that the ciliary bundles rest within a trough. The cupula that covers the neuromast is keel shaped, with its largest extension above the sensory strip and reaching to the top of the canal. The individual stereocilia are between 2-3 $\mu \mathrm{m}$ long, while the kinocilium is between $5-10 \mu \mathrm{m}$. The overall shape of the neuromast is elongated, with surface areas

between 0.01-0.06 $\mathrm{mm}^{2}$, dependent on canal location and on fish size (maturity).

Other species have canal neuromasts of different shapes. For example, the supra- 


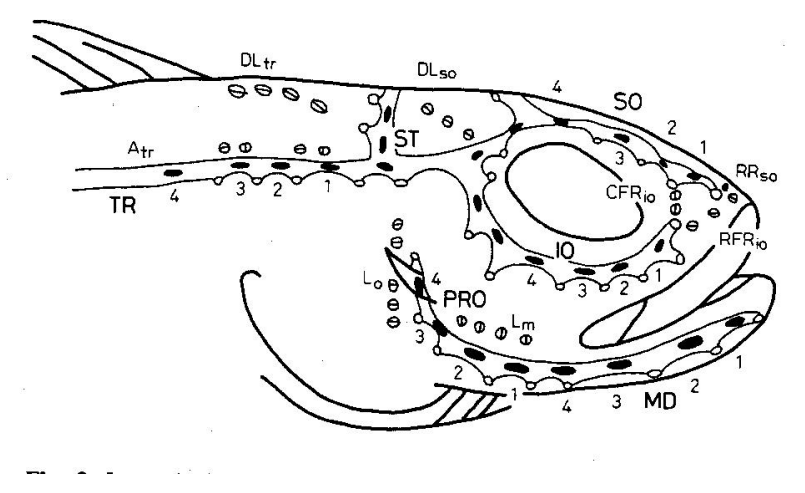

Figure 3-2: Lateral view of the distribution of canal (black dots) and superficial (open circles) neuromasts found in the mottled sculpin. Size of neuromasts is indicated by a line through the open circles. The position of canal neuromasts sampled for growth analyses are indicated by numbers. Neuromasts in the supratemporal canal and that portion of the main body canal extending from the orbital canals to the trunk canal were not sampled. Atr $=$ Trunk accessories; CFRio $=$ caudal replacement of the infraorbital canal; DLso $=$ dorsal line of the supraortial canal; DLtr $=$ dorsal trunk line; $\mathrm{IO}=$ infraorbital canal; $\mathrm{Lm}=$ mandibular line; $\mathrm{Lo}=$ opercular line; $\mathrm{MD}=$ mandibular canal; $\mathrm{PRO}=$ preopercular canal; $\mathrm{RFRio}=$ Rostral replacement of the infraorbital canal; RRso= Rostral replacement of the supraorbital canal; $\mathrm{SO}=$ supraorbital canal; $\mathrm{TR}=$ trunk canal. Diagram and caption from Janssen et al. (1987, Figure 2). Reprinted with permission of S. Karger AG, Basel.

orbital canal neuromast of the ruffe has a rounded base $0.6 \mathrm{~mm}$ in diameter, and extends to a height of $0.7 \mathrm{~mm}$ in a $1 \mathrm{~mm}$ diameter canal (van Netten, 2006). How each neuromast will respond to the passing fluid within the canal will be species and canal specific. However, as will be seen, approximations can lead to useful models.

In a seminal work, Denton and Gray (1983) showed that the fluid displacements within a canal relative to the fish body motion are linearly related to the fluid velocities outside the canal relative to the fish body motion, which means the fluid velocities in the canal are proportional to the fluid accelerations outside the canal. This is equivalent to saying that water motion within the canal is determined by the pressure gradient between pore openings (Kalmijn, 1988).

At the surface of the skin, the velocity of the water is exactly equal to the velocity of the skin. This is called the no-slip condition, and it exists because at the surface the molecular forces keep the water in contact with the skin. Between the skin and 


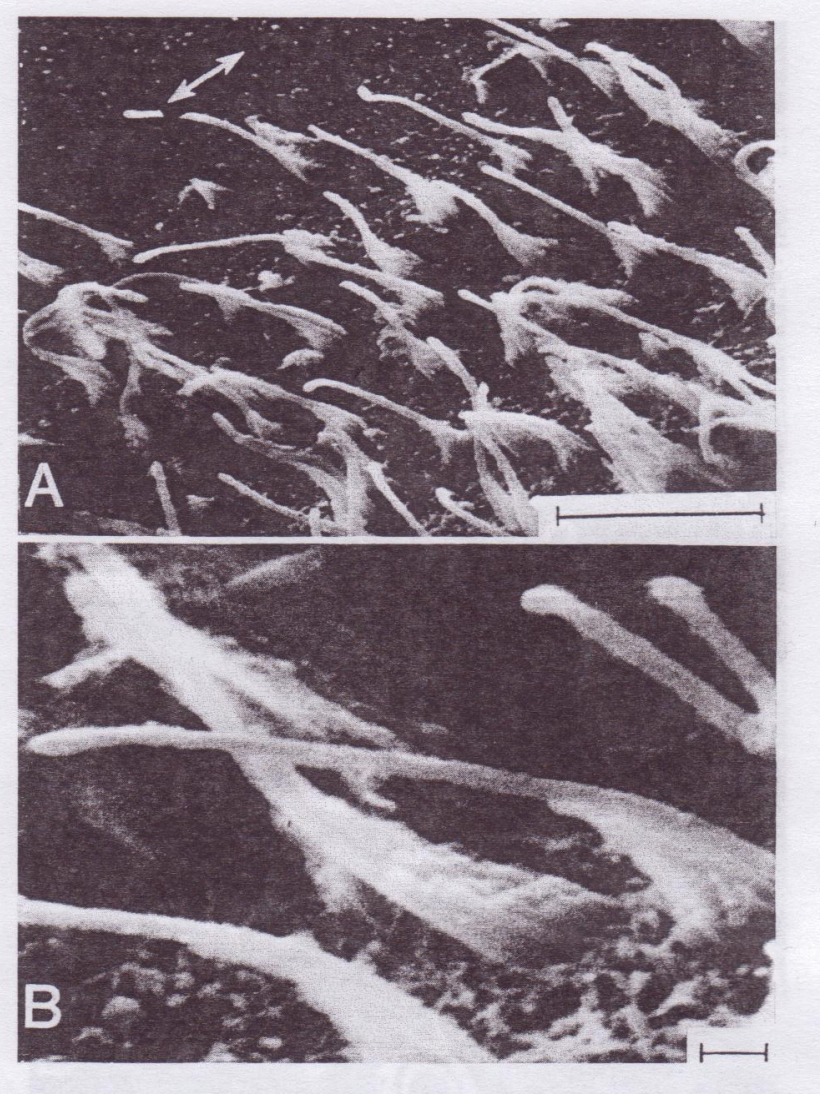

Figure 3-3: Scanning electron micrographs of apical ciliary bundles on the canal neuromast shown in figure $1 \mathrm{~A}$. A Low magnification showing the orientation of ciliary bundles along the canl axis (arrows). B Higher magnification showing the opposite polarity of bundles on adjacent hair cells. Scale bars: (A) $10 \mu \mathrm{m}$, (B) $1 \mu \mathrm{m}$. Photograph and caption from Janssen et al. (1987, Figure 6). Reprinted with permission of S. Karger AG, Basel.

the free-stream velocity, where inertial forces dominate, a transition zone called the boundary layer exists to transition the velocities. In the boundary layer, viscous forces dominate, and there is generally strong shear. For thin boundary layers, the pressure field penetrates almost undisturbed to the skin surface. This means that the pressure at the canal pore openings should be approximately equal to the pressure at points normal to the surface just outside the boundary layer.

Kalmijn (1988) modeled the canal / canal neuromast system as a damped harmonic oscillator, driven by fluid drag. He argued that it was the canal fluid velocity which is the relevant stimulus to the canal neuromast, since the cupula would bend 
from the drag force, which is proportional to the relative velocity between the cupula and oscillating fluid. Using Kalmijn's notation, $m$ the mass of the gelatinous cupula, $b$ the viscous force per unit velocity, $k$ the restoring force per unit cupular displacement, $v^{\prime} \cos \omega t$ the velocity of the driving fluid within the canal, and $x$ the cupular displacement, he arrives at

$$
m \frac{d^{2} x}{d t}+b \frac{d x}{d t}+k x=b v^{\prime} \cos \omega t
$$

The steady state solution, in terms of cupular displacement per canal fluid velocity, and the corresponding phase lag, is

$$
\frac{x(\omega)}{v^{\prime}}=\frac{\gamma}{\omega_{0}^{2}} \frac{\omega_{0} / \omega}{\left[\left(\omega_{0} / \omega-\omega / \omega_{0}\right)^{2}+\left(\gamma / \omega_{0}\right)^{2}\right]^{1 / 2}}, \quad \theta=\tan ^{-1}\left(\frac{\gamma / \omega_{0}}{\omega_{0} / \omega-\omega / \omega_{0}}\right)
$$

where $\gamma=b / m$ and $\omega_{0}^{2}=k / m$. This system is critically damped for $\gamma=2 \omega_{0}$, and underdamped or overdamped for $\gamma$ less than or greater than $2 \omega_{0}$, respectively (Kalmijn, 1988). Figure 3-4 shows three cases: $\gamma<<\omega_{0}$ (underdamped), $\gamma=2 \omega_{0}$ (critically damped), and $\gamma>>\omega_{0}$ (overdamped). The figure plots the range of frequencies, from $\omega<<\omega_{0}$, through $\omega=\omega_{0}$, to $\omega>>\omega_{0}$. For $\omega<<\omega_{0}$, the beginning amplitude $x(\omega) / v^{\prime} \approx \gamma / \omega_{0}^{2}$, while the phase approaches zero. When $\omega=\omega_{0}$, the amplitude $x(\omega) / v^{\prime} 1 / \omega$ scaled by $\gamma / \omega_{0}^{2}$, and the phase is -90 degrees for all cases. When $\omega>>\omega_{0}$, the amplitude decreases as $x(\omega) / v^{\prime} 1 / \omega^{2}$ scaled by $\gamma / \omega_{0}^{2}$, and the phase approaches -180 degrees. For $\gamma<<\omega_{0}$, the viscous damping/driving is much less than the restoring force, and in essence this system typifies a mass-spring system, showing resonance at $\omega_{0}$. At the other extreme, $\gamma>>\omega_{0}$, the system is dominated by viscous forces which are competing to both drive and damp the system. For $\gamma \approx 2 \omega_{0}$, inertia and viscous forces compete equally, and the displacement is still in phase for $\omega<<\omega_{0}$

Although values for $m, k$, and $b$ where not provided, Kamijn assumes the relationship $\omega<<\omega_{0}$ holds for biologically relevant cases. He then arrives at a general solution for the cupular displacement as related to the outside water volume fluid 

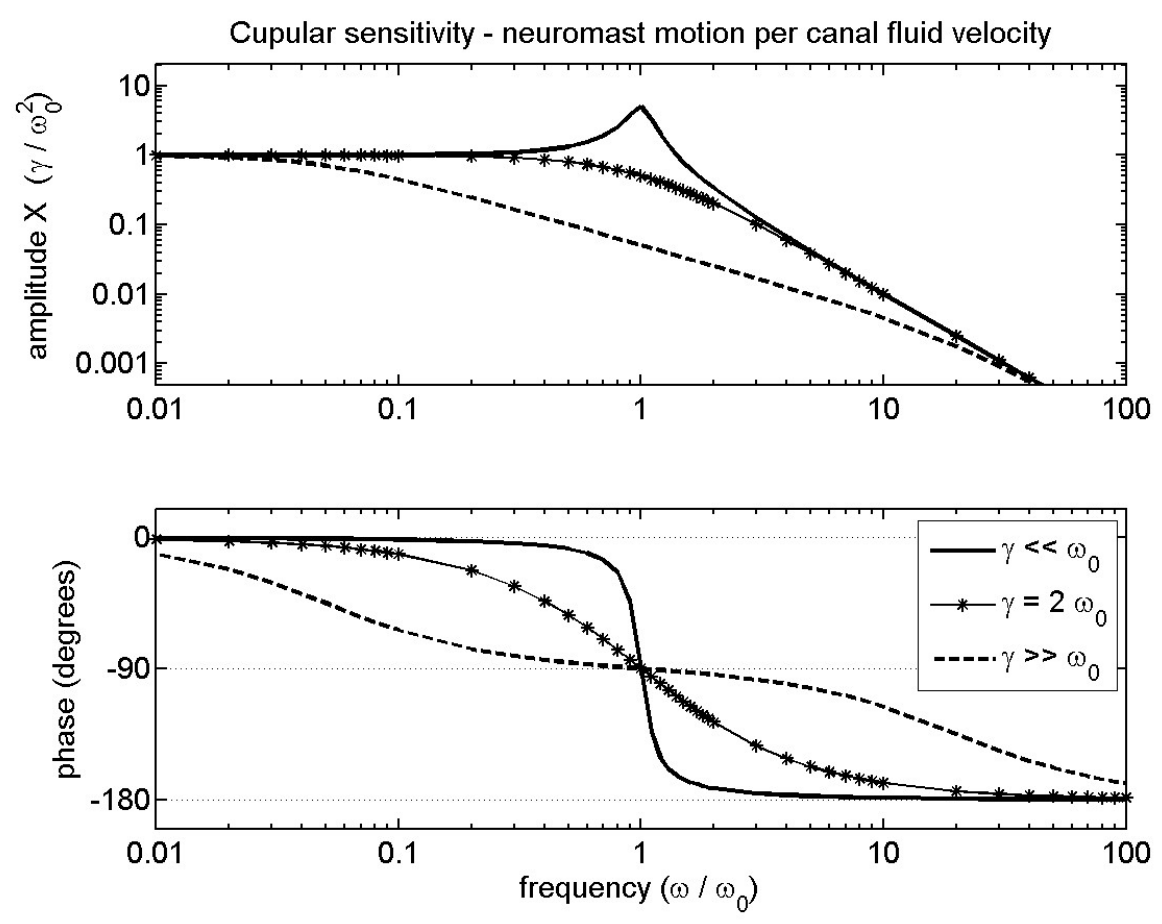

Figure 3-4: Three cases of a driven, damped harmonic oscillator, representing the cupular displacement per canal fluid velocity (Kalmijn's neuromast model, 1988). The plot shows the transition from a mass-spring system $\left(\gamma<<\omega_{0}\right)$ to that of a viscously driven and damped system $\left(\gamma>>\omega_{0}\right)$. The phase is calculated based on the neuromast displacement motion relative to canal velocity.

velocity,

$$
x \cong(b / k) v\left(\omega / \omega^{\prime}\right)^{s} \cos \omega t
$$

where $\omega^{\prime}$ and $s$ are properties inherent to the particular system under study, and $v$ is the fluid velocity of the outside water volume. Here $\omega_{0}$ and $\omega^{\prime}$ are different quantities, with the previous relating to the neuromast system and the latter relating the canal velocity to the outside water volume velocity. For the canal / canal neuromast system, Kalmijn gives $s \approx 1$, and $f^{\prime}=\omega^{\prime} / 2 \pi \approx 100 \mathrm{~Hz}$, which means the displacement of the cupula is in phase with the acceleration of the fluid outside the canal relative to the body/canal acceleration (as experimentally verified by Denton and Gray, 1983). For this to hold true, $\omega<\omega_{0}$ for an underdamped system, $\omega<<\omega_{0}$ for a critically damped system, and $\omega$ must be orders of magnitude less than $\omega_{0}$ for an overdamped 
system. The larger $\omega_{0}$ is in absolute terms, the easier these conditions can be met for the various systems.

This canal-neuromast system model was improved by van Netten (2006), who took the additional steps of modeling a frequency dependent drag force and adding a fluid buoyancy force. The pressure field needed to oscillate the fluid is what causes this buoyancy force on the sphere. Using van Netten's fluid displacement term, $W$, the model is

$$
m \frac{d^{2} x}{d t}=-k x+D\left(\frac{d x}{d t}-\frac{d W}{d t}\right)+m \frac{d^{2} W}{d t},
$$

where $b=-D$ and $v^{\prime}=i \omega W_{0}$ from Kalmijn's terminology, and $W(t)=W_{0} \exp (i \omega t)$. For his model, van Netten approximated the shape of the canal neuromast as a sphere and assumed that the vibratory amplitude of the fluid is much much less than the length of the body. Under these conditions, the flow around the boundary layer of the sphere reduces to Stokes flow (i.e. the nonlinear term from the Navier-Stokes equation is considered insignificant), and calculation of the drag integrated over the surface can be determined from an analytical solution. A second assumption made by this model is that the fluid flow within the canal has a uniform velocity profile. Solving for the steady state solution, and writing the solution in terms of the ratio of cupular displacement to excitatory fluid velocity amplitude, $V_{0}=i \omega W_{0}$, the cupular sensitivity is

$$
S(f)=\frac{1}{2 \pi f_{t}} \frac{1+\frac{1}{2} \sqrt{2}(1+i)\left(\frac{f}{f_{t}}\right)^{\frac{1}{2}}+\frac{1}{3} i \frac{f}{f_{t}}}{N_{r}+i \frac{f}{f_{t}}-\frac{1}{2} \sqrt{2}(1-i)\left(\frac{f}{f_{t}}\right)^{\frac{3}{2}}-\frac{1}{3}\left(\frac{f}{f_{t}}\right)^{2}} .
$$

The two parameters are the transition frequency, $f_{t}$, and the resonance number, $N_{r}$. The transition frequency describes the transition from viscous dominating forces to inertial dominating forces (a.c. Reynolds number, $R e_{\text {a.c. }}=f / f_{t}$ ), and is given as $f_{t}=\mu /\left(2 \pi \rho a^{2}\right)$, where $\mu$ is the viscosity of the canal fluid, $\rho$ is the density of the fluid, and $a$ is the radius of the sphere. The resonance number determines whether or not there will be resonance properties of the cupula in the canal fluid, and at what frequencies. It has an additional term, $K$, the sliding stiffness which is proportional 
to the restoring force, and is given as $N_{r}=K a \rho /\left(6 \pi \mu^{2}\right)$.

To see the effects of resonance displayed by the canal neuromast, it is useful to compare van Netten's model (equation 3.5 to that of Kalmijn's equation 3.2). From van Netten (2006), the canal neuromast of the ruffe has $N_{r}=64$ and $f_{t}=10.6$ $\mathrm{Hz}$, while corresponding values for the African knife fish are $N_{r}=20$ and $f_{t}=77$ Hz. Trying to calculate a transition frequency and resonance number for the Mottled sculpin would not be appropriate at this time. For the Mottled Sculpin, the keelshaped cupula has similar length and width portions as the sensory strip (see figure 3-3), and extends to roughly the top canal which has a cross-sectional area of about $9 \mathrm{~mm}^{2}$ (Janssen et al., 1987). Modeling the cupula of the sculpin is dealt with specifically in section 3.1.3.

To use Kalmijn's model, the restoring force and drag need to be determined. The restoring force, obtained by using the formulas for the transition frequency and resonance number, are $0.11 \mathrm{~N} / \mathrm{m}$ for the ruffe and $0.09 \mathrm{~N} / \mathrm{m}$ for the African knife fish. The viscosity of the canal fluid was taken to be $0.005 \mathrm{~kg} / \mathrm{m}-\mathrm{s}$ (van Netten and Kroese, 1987). The frequency dependent drag over a sphere (canal neuromast) is given as (Stokes, 1851; van Netten 1991)

$$
D(\omega) \frac{d X}{d t}=-6 \pi a \mu\left[\left(1+\frac{a}{\delta(\omega)}\right)+i \frac{a}{\delta(\omega)}\left(1+\frac{2 a}{9 \delta(\omega)}\right)\right] \frac{d X}{d t}
$$

where $\delta(\omega)=\sqrt{2 \mu / \rho \omega}$ was previously defined (equation 2.2 , with $\nu=\mu / \rho$ ) as the height of the boundary layer to within an order of magnitude (Schlichting, 1979), and $d X / d t$ is the velocity of the sphere. Kalmijn's model does not address a frequency dependent drag, so the median value from figure 3-5 will be used. This is in fact an arbitrary choice. For the range of values shown, there is only a slight scaling effect.

The models show significant differences in both amplitude and phase at low frequencies (see figure 3-6), but are comparable at frequencies above about $300 \mathrm{~Hz}$. The figure shows that for the values of the biological parameters chosen, the amplitude and the phase using Kalmijn's model follows for an overdamped system $\left(\gamma>>\omega_{0}\right)$ with $\omega$ within an order of magnitude of $\omega_{0}$. This means that the canal neuromast 


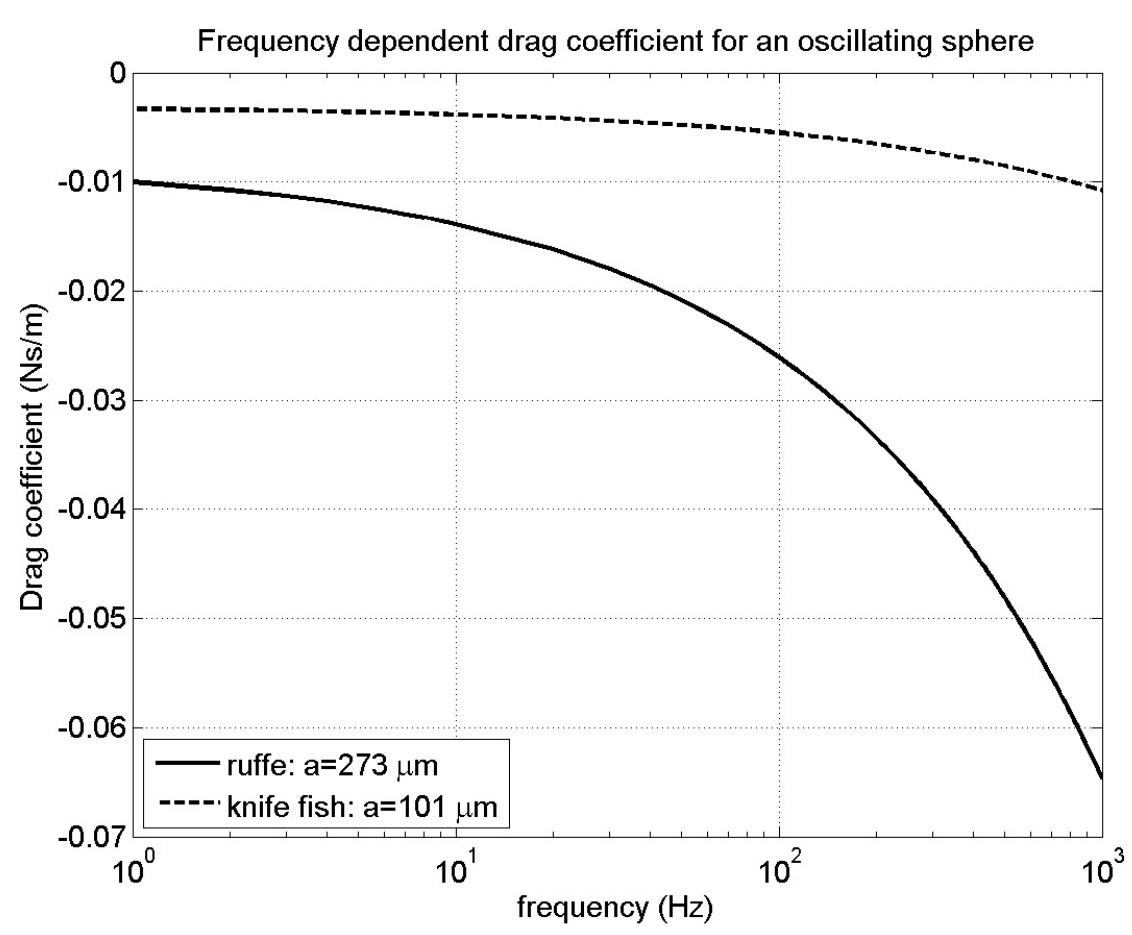

Figure 3-5: Canal neuromasts for the ruffe and African knife fish modeled as an oscillating sphere (van Netten, 2006). For a sphere with small amplitude oscillations relative to its radius, a frequency dependent boundary layer forms where viscous forces dominate inertial forces. The drag coefficient is affected by the size of the sphere, and is frequency dependent (Stokes, 1851).

displacement would not be in phase with the outside water volume acceleration. van Netten's model shows that there is a slight phase lead that increases up to the point of resonance, and then falls away at higher frequencies, overall acting like an underdamped system. van Netten's (2006) data for the ruffe and African knife fish validate his model for the frequency ranges presented (approx. 40-250 Hz, and approx. 90-800 $\mathrm{Hz}$, respectively). For biological cases where $N_{r}<<1$, van Netten shows that his model reduces to a purely velocity detecting system, which is Kamijn's model.

Though both models assume a uniform velocity within the canal as an approximation, both also model its frequency dependency. The actual velocity in the subdermal canal will not be uniform, but will have various velocity profiles. This is due to the fact the boundary layer thickness against the wall changes based on frequency. At low frequencies, the boundary layer thickness will extend from the wall all the way to 

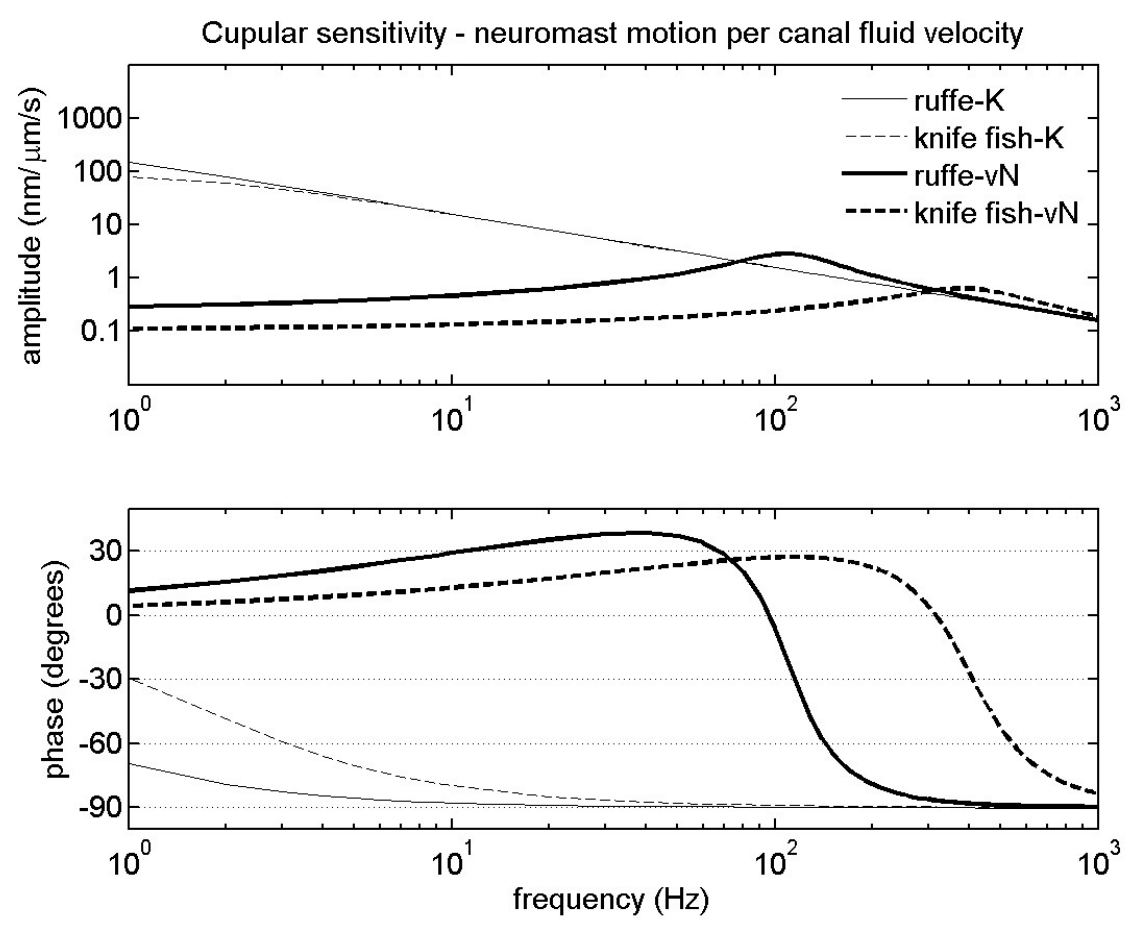

Figure 3-6: A comparison between a velocity detecting canal neuromast system (KKalmijn), and a system based on a resonance number (vN-van Netten), for the ruffe and the African knife fish (data from van Netten, 2006). The plot shows the amplitude and phase of the canal neuromast displacement relative to the canal fluid velocity moving past (similar to figure 3-4). Note the scaling differences in the units of amplitude.

the channel center, and the velocity profile will be parabolic in shape with the fastest velocity at the center. At higher velocities, the boundary layer shrinks closer to the wall, so that the maximum channel velocity is no longer at the center but falls in a region around the edge of the boundary layer (see figure 3-7).

Denton and Gray (1983) compared the properties of subdermal canal channels for the sprat (in particular) to that of capillary tubes. Though the canal channels were elliptical in shape, with heights of about $250 \mu \mathrm{m}$ and width of $100 \mu \mathrm{m}$, the velocity amplitude and phase characteristics matched those of a capillary tube of similar crosssectional area and oriented in the same direction. Flow inside a capillary (or pipe) 


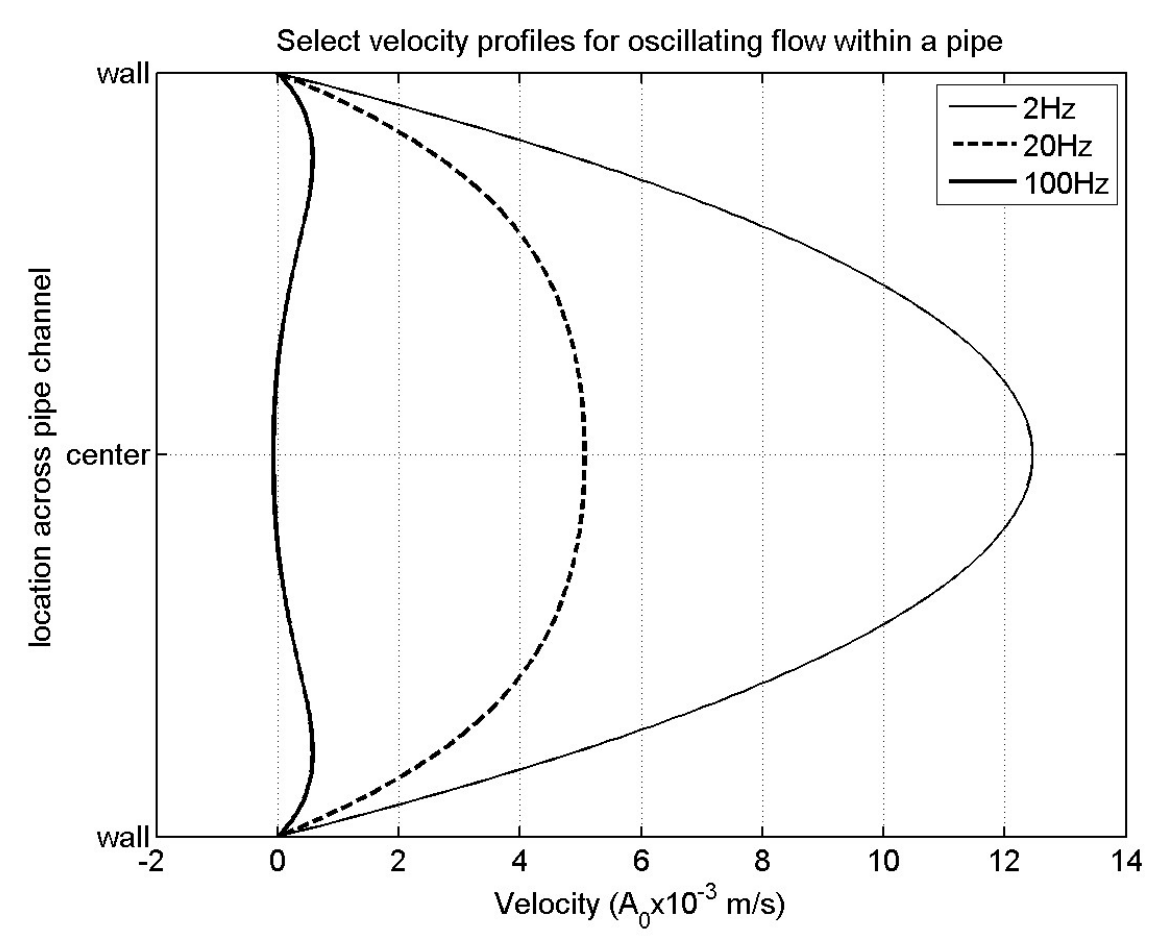

Figure 3-7: Instantaneous velocity profiles inside a pipe for selected frequencies of an oscillating pressure gradient (after Schlichting, 1979). Each of the velocity profiles change in shape throughout their oscillation period (see figure, and has a different phase from the acceleration signal (bottom plot) which drives it. The radius of the pipe is $0.5 \mathrm{~mm}$, and the viscosity is $0.005 \mathrm{~kg} / \mathrm{m}-\mathrm{s}$.

has an analytical solution, given by Schlichting (1979)

$$
u(r, t)=-i \frac{A_{0}}{\omega} e^{i \omega t}\left[1-\frac{J_{0}(r \sqrt{i \omega / \nu})}{J_{0}(R \sqrt{i \omega / \nu})}\right],
$$

where $A_{0}$ is the amplitude of the acceleration, which is directly related to the pressure gradient through the tube, $(-1 / \rho) \partial p / \partial x=A_{0} e^{-i \omega t}, r$ is the distance from the channel center towards the pipe walls, $R$ is the radius of the pipe, $\nu$ is the kinematic viscosity of the fluid in the pipe, and $J_{0}(x)$ is a Bessel function of the first kind, zeroth order. Borrowing from Schlichting's diagram, figure 3-8 presents what a velocity profile with $100 \mathrm{~Hz}$ oscillations would look like inside a pipe.

van Netten uses Schlichting's solution for oscillating flow within a neuromast 


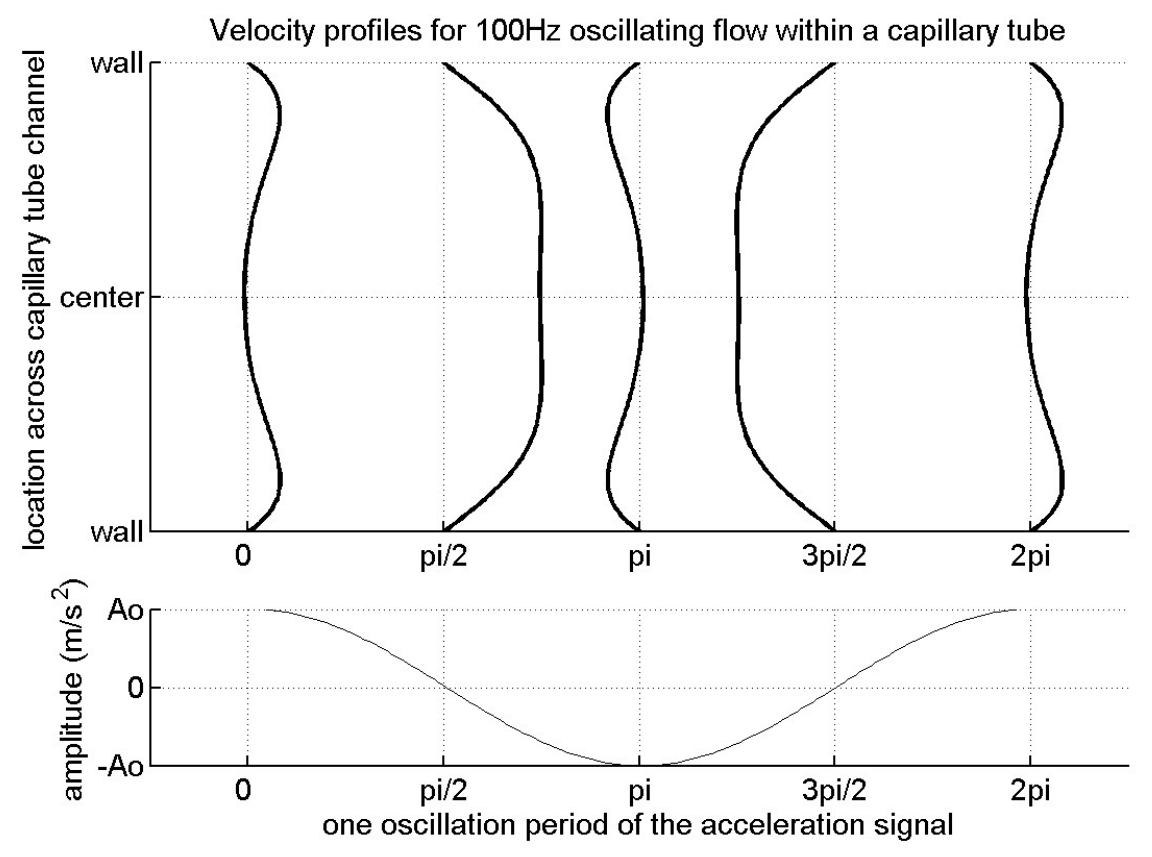

Figure 3-8: Velocity profile inside a capillary tube with an oscillating pressure gradient, after Schlichting (1979). The velocity profile (top plot) changes in shape throughout it's oscillation, and has a different phase from the acceleration signal (bottom plot) which drives it. The capillary tube has a radius of $0.5 \mathrm{~mm}$, and the viscosity is 0.005 $\mathrm{kg} / \mathrm{m}$-s. Note the scaling differences in the units of amplitude.

canal/pipe to determine the steady state transfer function from the inside canal velocity to the outside acceleration, $S_{\text {can }}(r=0, f)=u(0, f) / A_{0}$. By virtue of the physical restrictions of the canal dimensions, the canal acts as a low pass filter of frequencies. van Netten chooses the center of the subdermal canal for its filtering properties, and as figure 3-9 shows, the effects of the canal on smaller canal neuromasts is not terribly different (i.e. amplitude changes within an order of magnitude). However, changing the size of the canal has a significant effect on the filtering properties of the canal. As figure 3-10 shows, there is over two orders of magnitude difference for channel center velocity of the canal when the canal changes size from $0.2 \mathrm{~mm}$ to $10 \mathrm{~mm}$ in diameter, and the phase lag changes from 0 to 90 degrees, respectively. When the height of the neuromast is held fixed (i.e. $0.1 \mathrm{~mm}$ ) and the canal radius is changed, then the result has an amplitude pattern like seen in figure 3-10, but scaled according to the 

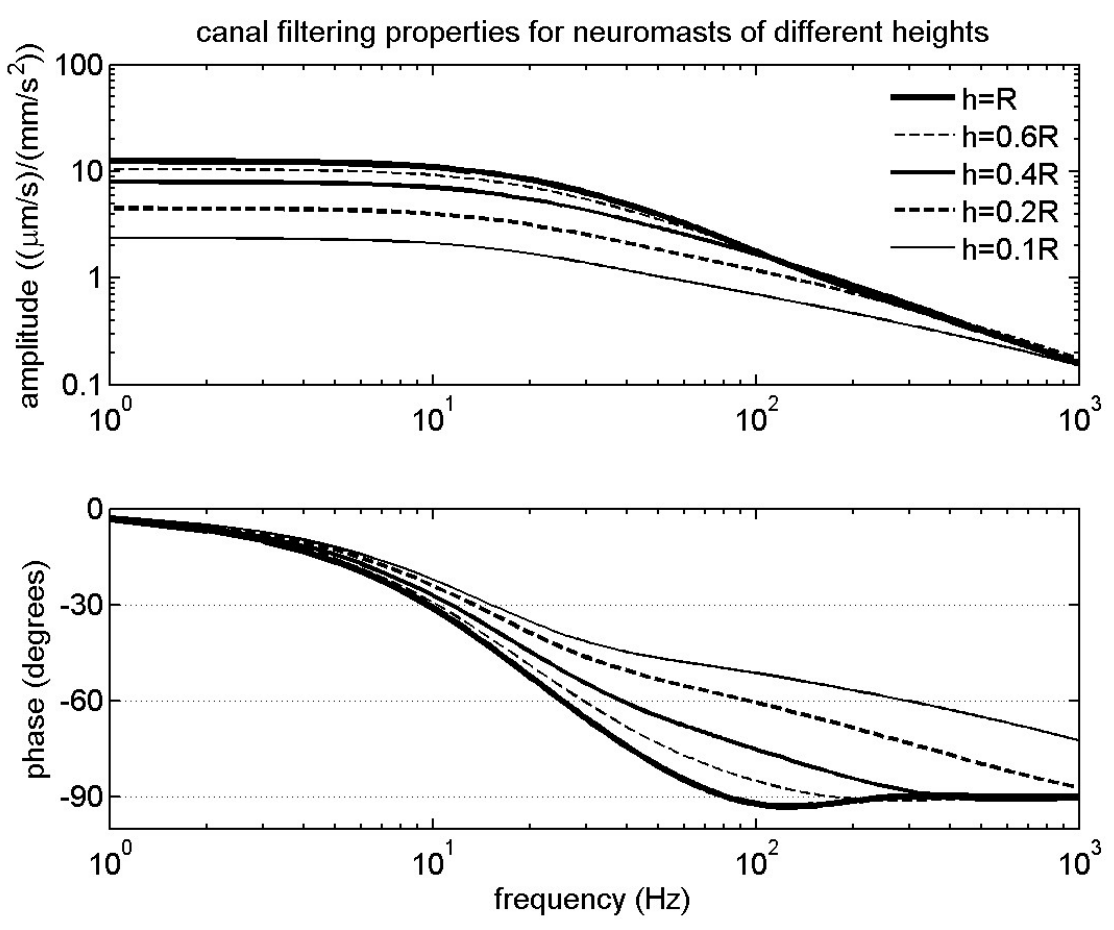

Figure 3-9: The subdermal canal, represented by a capillary tube, has low pass filtering characteristics. Canal neuromasts of varius height relative to the canal radius, $\mathrm{R}$, are compared in canal of radius $1 \mathrm{~mm}$ diameter. The viscosity of the canal fluid is $0.005 \mathrm{~kg} / \mathrm{m}-\mathrm{s}$, based on van Netten and Kroese (1987). Note the scaling differences in the units of amplitude.

amplitudes in 3-9. Likewise, the phase lag falls somewhere in between that of figure 3-10 and figure 3-9.

Barbier and Humphrey (2006) ran 2D and 3D numerical simulations of the flow within a subdermal canal of diameter $250 \mu \mathrm{m}$. Like Denton and Gray (1983), they found that the pressure within the canal depends only on the pressure gradient between the pore openings, and that each section of the canal (containing one neuromast) acts fairly independently of every other section. They simulated flow past two sizes of neuromasts, 50x50x100 $\mu \mathrm{m}$ and 150x150x15 $\mu \mathrm{m}$, which were immobile and fixed to the substrate. They showed that the canal sensitivity is roughly 1.9 $(\mu \mathrm{m} / \mathrm{s}) /\left(\mathrm{mm} / \mathrm{s}^{2}\right)$ for the flow just above the small neuromast (frequency unknown), and that the phase lag falls from 0 to just past 70 degrees over the frequency range from about 1-200 Hz. This result matches very closely in amplitude and phase the 

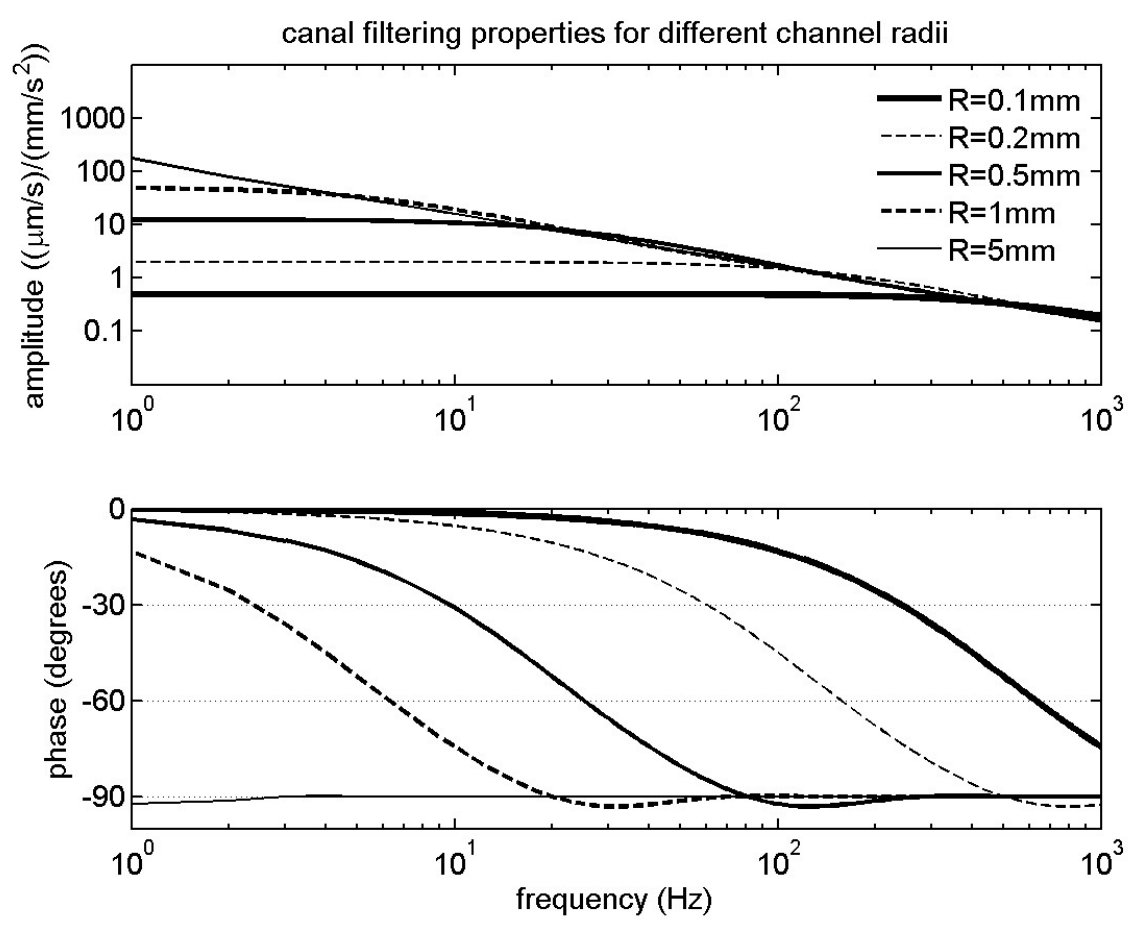

Figure 3-10: The effect that changing the capillary tube radius, $\mathrm{R}$, has on the channel center velocity of an oscillating flow is compared. The viscosity of the canal fluid is $0.005 \mathrm{~kg} / \mathrm{m}-\mathrm{s}$, based on van Netten and Kroese (1987). Note the scaling differences in the units of amplitude.

center velocity in a canal of larger diameter, $400 \mu \mathrm{m}$ (see figure 3-10). The conclusion is that a neuromast which more completely blocks the canal does alter flow patterns in the canal from what would be predicted by equation 3.7. The increased center velocity can be explained by the Bernoulli effect. Flow speed increases in the region of decreased canal area where the neuromast is located, which also leads to an increase in the drag force on the neuromast. The effects could best be predicted by performing a fluid-structure simulation, where the neuromast could bend with the passing fluid.

The cupular-canal sensitivity of the entire canal / canal neuromast system is equal the two transfer functions multiplied together, $S(f) * S_{\text {can }}(0, f)$. Figure 311 compares the cupular-canal sensitivity for the ruffe and African knife fish using van Netten's model and using Kalmijn's model. Using the values for the drag force and restoring force coefficients particular to the ruffe and African knife fish, Kalmijn's model behaves at system with $\gamma>>\omega_{0}$ and for $\omega<<\omega_{0}$, whereas van Netten's model 

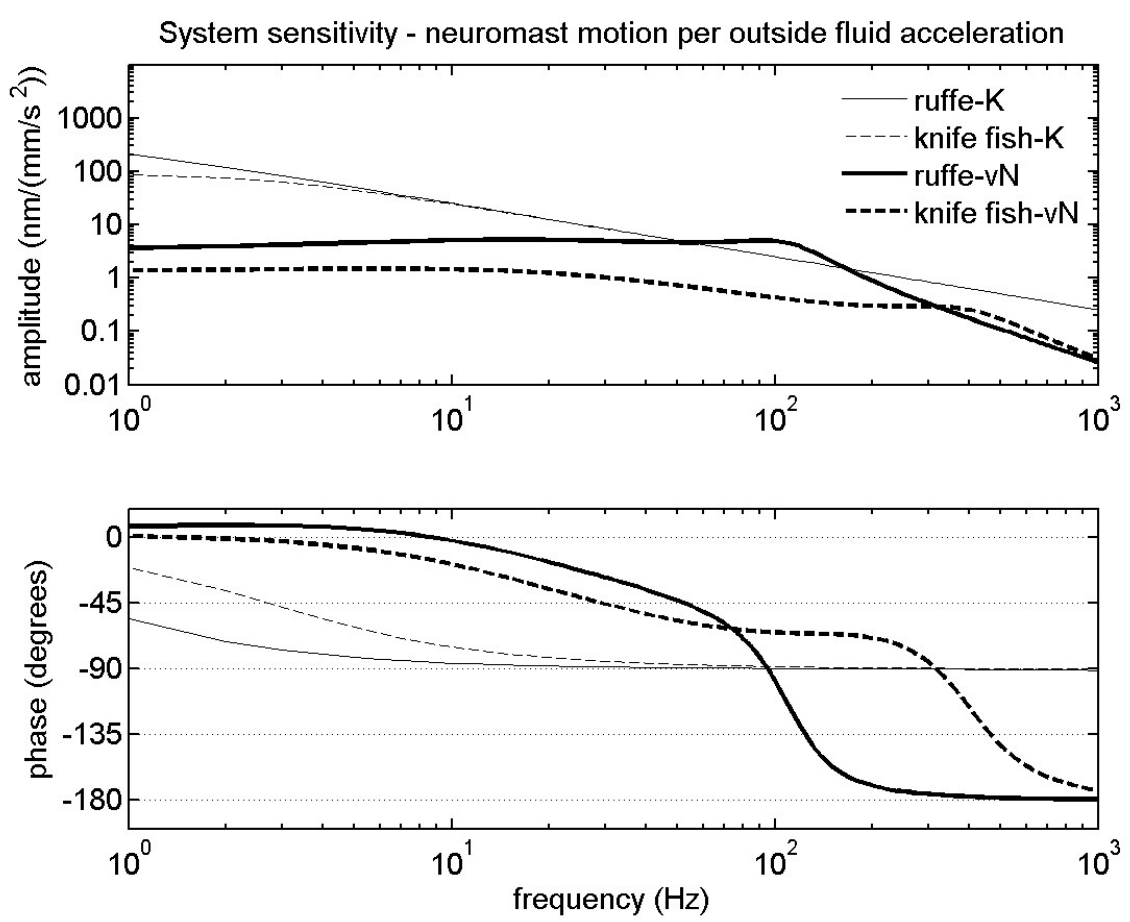

Figure 3-11: Cupular sensitivity of the canal neuromast system. The neuromast displacement per outside water volume acceleration is compared for Kalmijn's $(\mathrm{K})$ model and van Netten's (vN) model for the ruffe and the African knifefish. Kalmijn's model is based on a velocity detecting system only, where the resonance number is small $N_{r}<<1$, whereas van Netten's model allows for all values of the resonance number. The ruffe has $N_{r}=64$, and the African knife fish has $N_{r}=20$. The subdermal canal is assigned a radius of $0.5 \mathrm{~mm}$, and has a viscosity of $0.005 \mathrm{~kg} / \mathrm{m}-\mathrm{s}$. Note the scaling differences in the units of amplitude.

almost exactly matches Kalmijn's model if $\gamma \approx \omega_{0}$ and $\omega<<\omega_{0}$ (see figure $3-4$ ). The overall system behaves as Kalmijn predicted, but van Netten's model captures the process for a wider range of cupula-canal dynamics (van Netten, 2006).

The combined dynamics of the canal and canal neuromast have been shown to respond to the acceleration of the fluid relative to the fish body, which is driven by the pressure gradient (Denton and Gray, 1983). This is true for a wide range of frequencies, up to about $100 \mathrm{~Hz}$ (van Netten, 2006). However, the specific responses of the canal neuromast really depend on its size relative to the frequency dependent boundary layers that form on the surfaces of the canal, and also on the size of the canals. Amplitude and phase properties change significantly with canal size. Neuromasts of 
other shapes, such as the keel shape canal neuromasts of the Mottled sculpin, will also have an effect on the fluid-structure interaction within the subdermal canal.

\subsubsection{Superficial neuromasts}

Janssen et al. (1987) found that the superficial neuromasts of the Mottled sculpin where similar in shape to other clupeids, having a base less than $100 \mu \mathrm{m}$ in diameter, and with stereocilia (2-3 $\mu \mathrm{m}$ long) and kinocilium (5-10 $\mu \mathrm{m}$ long) like the hair cells of the canal neuromasts. They consist of far fewer hair cells than found in the canal neuromasts, and the epidermal tissue that surrounds it can get scarred and obscure the cilia. The cupula can be less than $50 \mu \mathrm{m}$ in height in larval stages (i.e. larval Zebrafish, McHenry and van Netten, 2007), while a typical size in adults is double that (Kalmijn, 1988). Even in adults there can be a range of neuromast sizes based on their particular body location (Janssen et al., 1987).

When there is relative water motion between the body and the surrounding fluid, a boundary layer develops where the motion of the fluid transitions from rest relative to the body to its full velocity in the surrounding flow. When the boundary layer thickness is a significant portion of the neuromast height, it is the dynamics within the boundary layer that governs the motion of the neuromast. Complicating this is the fact that the superficial neuromasts reside either partially or fully in the mucous layer covering the skin, which is considered a non-Newtonian fluid.

Kalmin (1988) used the same basic model for the superficial neuromasts as he did for the canal neuromasts (i.e. equation 3.1), but changed the relationship between the fluid velocity driving the neuromast and the outside water volume. Inside the boundary layer, the flow is reduced relative to the outside water volume according to $v\left(\omega / \omega^{\prime}\right)^{1 / 2}$, where $v$ is the outside water velocity, $\omega$ is the frequency, and $f^{\prime}=\omega^{\prime} / 2 \pi=$ $200 \mathrm{~Hz}$ is a scaling term for the superficial neuromast system of the particular fish being studied. He points out that this is really equivalent to the geometric mean

of the velocity and acceleration, since $v\left(\omega / \omega^{\prime}\right)^{1 / 2}=\left(v^{2} \omega / \omega^{\prime}\right)^{1 / 2}=(v a)^{1 / 2}\left(\omega^{\prime}\right)^{-1 / 2}$, 
where the acceleration is $a=v \omega$. For $\omega<<\omega_{0}$, his final model is

$$
x \approx \frac{b}{k}\left(\omega^{\prime}\right)^{-1 / 2}(v a)^{1 / 2} \cos \omega t
$$

where $b$ is the viscous force per unit velocity, and $k$ is the restoring force per unit cupular displacement.

Dinklo (2005) adapted van Netten's model for the canal / canal neuromast system for use with superficial neuromasts. He kept the same model for cupular sensitivity, but instead of a frequency dependent canal velocity profile, he used a frequency dependent boundary layer profile. This boundary layer model, which relates the fluid velocity inside the boundary layer to the freestream velocity outside the boundary layer, is given by Lamb (1932) as

$$
\frac{u}{U_{0}}=-i\left[1-e^{-(1+i) y / \delta(\omega)}\right] e^{i \omega t}
$$

where $\delta(\omega)=\sqrt{2 \mu / \rho \omega}$ was previously defined as an approximate measure of the boundary layer thickness, $y$ is the height above the surface, $i=\sqrt{-1}$ denotes complex notation, and $-i U_{0} e^{i \omega t}$ is the uniformly oscillating fluid velocity well outside the boundary layer. Equation 3.9 can be used without the $-i$, which changes the outside fluid velocity to $U_{0} \cos \omega t$.

The nature of the boundary layer that develops is to have a high pass filtering effect on the fluid velocity within the boundary layer, relative to the fluid outside of it. Figure 3-12 shows that this effect is more prominent the smaller the neuromast height is relative to the boundary layer height. For a neuromast of $100 \mu \mathrm{m}$, its tip would experience about a $10 \%$ reduction in velocity at $1 \mathrm{~Hz}$, and further reductions at lower frequencies.

Dinklo's model is an oscillating sphere in a boundary layer, given by $S(f) *$ $S_{b l}(y, f)$, where $S(f)$ is van Netten's model for cupular sensitivity, and $S_{b l}(y, f)=$ $u(y, f) / U_{0}$ is the boundary layer sensitivity at some appropriate height $y$. When examining the superficial neuromasts of larval zebrafish, Dinklo found that $y \approx 34 \mu \mathrm{m}$ gave a best fit to the data, which was just above the tips of the cupula he measured 

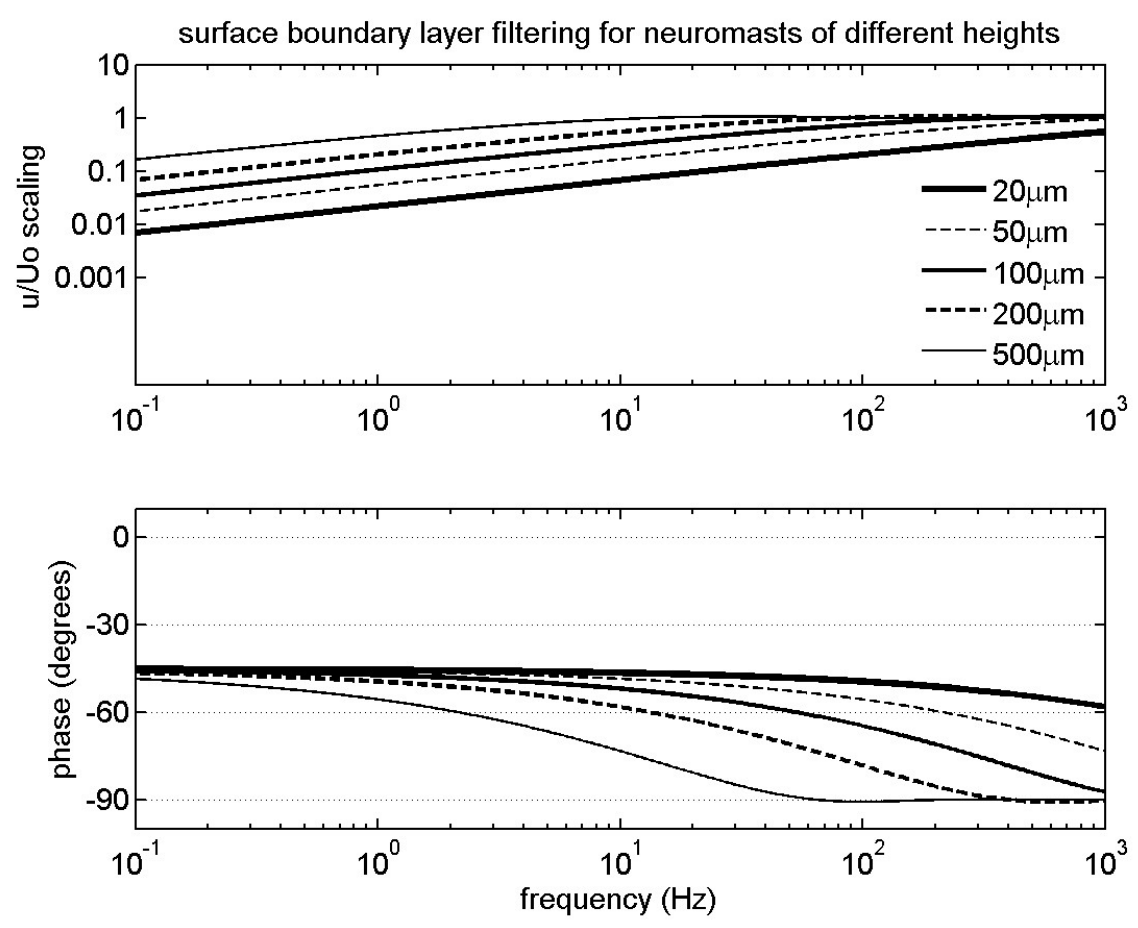

Figure 3-12: The oscillations of a uniform fluid over a flat plate have high pass filtering characteristics for the fluid velocity within the boundary layer, $u$, relative to the outside fluid velocity, $U_{0}$. Heights of superficial neuromasts are listed in the key. Superficial neuromasts whose heights are small relative to the height of the boundary layer, $\delta(\omega)=\sqrt{2 \mu / \rho \omega}$, experience a greater reduction in velocity.

(19-28 $\mu \mathrm{m})$. This model predicted a best sensitivity close to a frequency of $11 \mathrm{~Hz}$, soon after which the sensitivity falls off at $-20 \mathrm{~dB} / \mathrm{dec}$. The amplitude of cupular sensitivity to outside fluid velocity predicted by the model very closely matched the mean of the measured results, but the phase only matched for frequencies greater than about $30 \mathrm{~Hz}$.

Applying van Netten's canal neuromast model, which is based on a sliding displacement, to the superficial neuromast, which pivots at its base, seems to captures much of the relevant dynamics. This is likely because in each model, one point on the cupula and one point in the flow is used to represent the entire dynamics. For the superficial neuromast, the tip displacement behaves as if it slides back and forth. However, the fact that the modeled phase does not match the actual phase hints at more complicated dynamics. In particular, the model breaks down because the ve- 
locity profile within the boundary layer has phase dependencies based on the height above the surface relative to the boundary layer height. A real cupula will integrate the phase differences along its height. Secondly, a real cupula has material properties that change along its height (McHenry and van Netten, 2007).

Humphrey et al. (1993) developed an analytical and numerical model for the full motion response of a single cylindrical hair cell on a curved surface immersed in an oscillating fluid flow. The model takes into account the variation in the velocity profile along the length of the hair, and is given by Humphrey et al. (2001) as

$$
I_{e f f} \ddot{\theta}+R_{e f f} \dot{\theta}+S \theta=4 \pi \mu G \int_{0}^{L} V_{F} y d y+\left(\frac{\pi \rho d^{2}}{4}-\frac{\pi^{2} \mu G}{g \omega}\right) \int_{0}^{L} \dot{V}_{F} y d y
$$

where $I_{e f f}$ is the effective moment of inertia, $R_{e f f}$ is the effective damping constant, $S$ is the torsional restoring constant, $G$ and $g$ are terms related to flow around a cylinder, $V_{F}$ is the velocity of the fluid, $y$ is the distance along a hair of length $L$ from its base, $d$ is the diameter of the hair, $\mu$ and $\rho$ are the viscosity and density of the fluid, $\omega=2 \pi f$ is the angular frequency, $\theta$ is the angular deflection of the hair, and derivatives are with respect to time. The terms $g$ and $G$ are obtained from Stokes' (1851) solution for the drag around an oscillating cylinder in an unbounded medium, and are valid for $a / \delta(\omega)<<\sqrt{2}$. This is a special case of the full drag solution for all frequencies. (See Humphrey et al., 2000, for more details and for the complete definitions of $g$ and $G$. See Appendix A for Stokes' general solution to the problem of an oscillating fluid past a cylinder.)

Using a uniformly oscillating flow past a flat surface, the velocity within the boundary layer is given by equation 3.9, and equation 3.10 admits an analytical solution (see Humphrey et al., 2001). The frequency dependent solution depends on the determination of two physiological measurements, the hair torsional restoring constant, $S$, and the damping constant, $R$ ( of $R_{e f f}=R+R_{\mu}$ ). This is because all other constants are determined by the hair geometry or frequency, and are apriori calculable. The model shows that surface hair cells possess resonance frequencies that decrease with an increase in hair cell height, and that maximum deflection angle increases approx- 
imately linearly with hair cell height and increases linearly with the far field velocity amplitude of the oscillating fluid (Humphrey et al., 2001).

The hair torsional restoring constant, $S$, and the damping constant, $R$, are not easy to obtain for hairs in water. Humphrey et al. (1993) present one method of obtaining these parameters, whereby the phase difference between the driving freestream velocity and the corresponding angular displacement is measured at two sets of frequencies. They then provide an analytical equation for solving for the two unknowns using two equations. Another method consists of using the known resonance frequency of the system under study, and then at high enough frequencies, a series of $S$ - $R$ pairs can be determined from an approximate solution (Humphrey et al., 2001). Constructing a couple of series of these $S$ - $R$ pairs for hairs of different lengths should lead to an overlap region where the values are similar, which is based on the assumption that $S$ and $R$ are fundamental mechanical properties of the hairs, and should be fairly uniform for the hairs under study.

Another method for determining the $S-R$ values is to directly measure one. McHenry and van Netten (2007) measured the flexural stiffness of the superficial neuromasts of larval zebrafish. They found that the neuromast consists of stiff lower region and a flexible upper region (see figure 3-13). In the lower region, the cupula is supported by the tall kinocilum from the hair cells, whereas the in the upper region, the cupula is does not have rigid structures. For an individual kinocilum, they found the flexural stiffness (which is equal to Young's modulus, E, times the second moment of area, $I$ ) to be $2.4 \times 10^{-21} \mathrm{Nm}^{2}$ (note: the article abstract reports this number a factor of 10 less, but this is not the case throughout the article). Using their formula relating the force required, $F$, to deflect of beam of length, $L$, a distance of $\delta, F=3(E I) \delta / L^{3}$, the corresponding torque, $F L$, can be set equal to the hair torsional restoring constant times the angle of deflection, $S \theta$. This yields $S=3 E I / L$ per kinocilium, since $\delta / L=\theta$.

To compare the three superficial neuromast models (Kalmijn, Dinklo, and Humphrey et al.) for the superficial neuromast displacement per outside water volume velocity, specific parameters must be determined. Dinklo (2005) provides an empirically 
determined stiffness coupling of $K=6.4 \mu \mathrm{N} / \mathrm{m}$ for the larval zebrafish superficial neuromast, based on a model fit to the data using $f_{t}=6336 \mathrm{~Hz}$ and $N_{r}=0.0017$. The transition frequency was calculated directly using a cupular diameter of $10 \mu \mathrm{m}$, and the empirically determined model cupula height was $y=34 \mu \mathrm{m}$ (for equation 3.9). To use Kalmijn's model, the stiffness coupling remains unchanged, but the restoring force per unit velocity, $B$, needs to be calculated from the drag (equation 3.6). Again, using the median value over the frequency range of $1-1000, B=32.6 \mu \mathrm{Ns} / \mathrm{m}$. Finally, to use Humphrey et al.'s model, the hair torsional restoring constant, $S$, and the damping constant, $R$, need to be obtained. Using the flexural stiffness values from McHenry and van Netten (2007) for the larval zebrafish (of the same diameter and height of Dinklo, 2005), and assuming 10 hair cells per superficial neuromast, $S=10\left(3 \cdot 2.4 \times 10^{-21} \mathrm{Nm}^{2} / 34 \times 10^{-6} \mathrm{~m}\right) / \mathrm{rad}=2.1 \times 10^{-15} \mathrm{Nm} / \mathrm{rad}$. When the resonant frequency that gives maximum angular deflection, $\omega_{\text {res }(\theta)}$, is known, the Humphrey et al. (2001) show that $R$ can be determined from $S=I_{e f f} \omega_{r e s(\theta)}^{2}+R_{e f f}^{2} /\left(2 I_{e f f}\right)$. Using $\omega_{\text {res }(\theta)}=11 \mathrm{~Hz}$ determined by Dinklo (2005) for the larval zebrafish, and $S$ determined from McHenry and van Netten (2007), give $R=1 \times 10^{-17} \mathrm{Nms} / \mathrm{rad}$.

Figure 3-14 compares the three models (Kalmijn, Dinklo, Humphrey et al.) for the superficial neuromast displacement per outside water volume velocity. Kalmijn's model is in the overdamped mode $\left(\gamma>>\omega_{0}\right.$, see figure 3-4), and seems to represent an upper bound on the amplitude. However, its phase properties are a much closer approximation to Dinklo's (2005) data in the lower frequencies than Dinklo's own model. What is most surprising is that Dinklo's spherical model matches very closely Humphrey's cylindrical model. Although both models use Stokes' (1851) formulation of drag around an oscillating sphere and cylinder, respectively, only Humphrey's model integrates the boundary layer velocity profile over the cupula's surface. At least at small cupula heights and somewhat similar cupula volumes/masses, the two models appear interchangeable.

What these models show is that cupular displacement has a changing relationship to the outside water volume hydrodynamics. According to Kalmijn's model, with the parameters given for the larval zebrafish, the neuromast displacement is most 


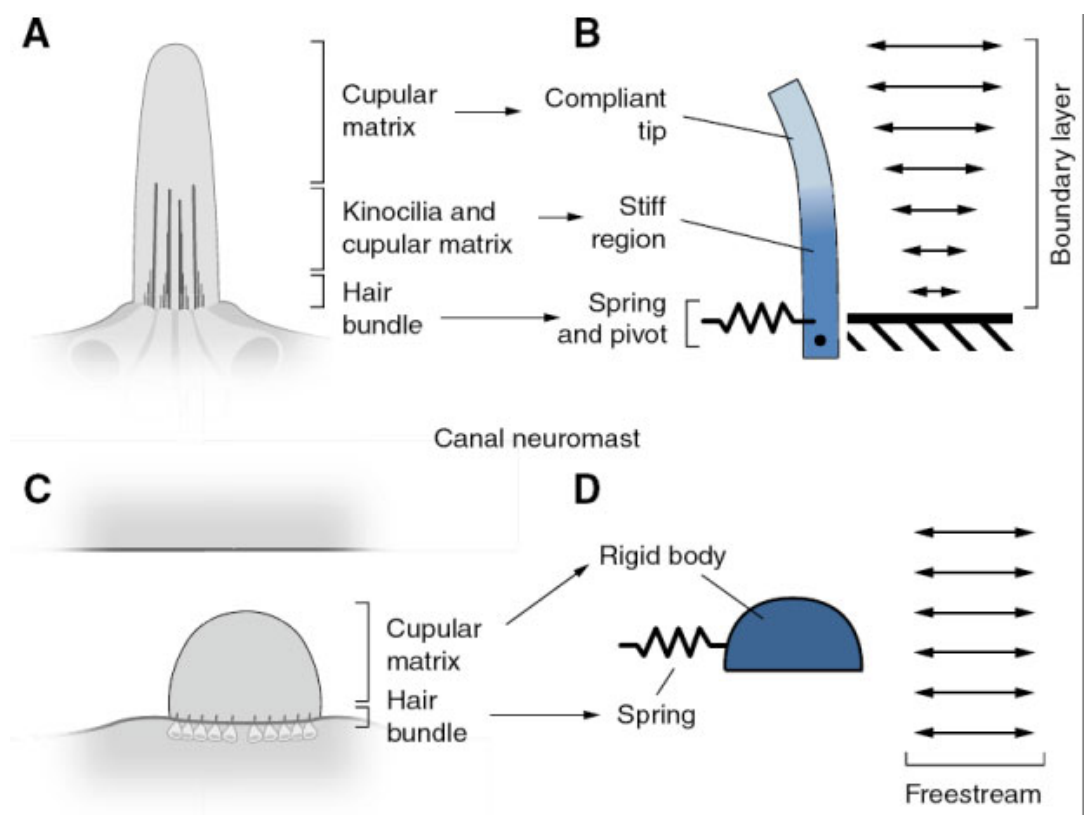

Figure 3-13: Proposed model for the mechanics of a superficial neuromast compared with the mechanics of a canal neuromast. (A) The major morphological features of a superficial neuromast each have a functional analog. (B) The proposed model consists of a flexible two-part beam that is driven by a boundary layer of water flow and coupled to a linear pivotal spring at its base. The presence of kinocilia causes the proximal part of this beam to be substantially stiffer than the distal part, which consists solely of matrix material. The morphology and mechanics of superficial neuromasts is contrasted with $(\mathrm{C}, \mathrm{D})$ the model for a canal neuromasts (van Netten and Kroese, 1987). (C) the same major anatomical features of a superficial neuromasts are present in the canal neuromast, but (D) kinocilia do not play a functional role that is distinct from the hair bundles, which collectively function as a linear spring. Furthermore, the cupula is modeled as a rigid hemispherical body that is coupled to the hair bundles. The freestream flow within the canal is not greatly influenced by boundary layer dynamics for the frequencies to which these neuromasts are sensitive (van Netten, 2006) and may therefore be modeled as a uniform freestream. Diagram and caption from McHenry and van Netten (2007), Figure. 7. Reproduced with permision from the Journal of Experimental Biology. 

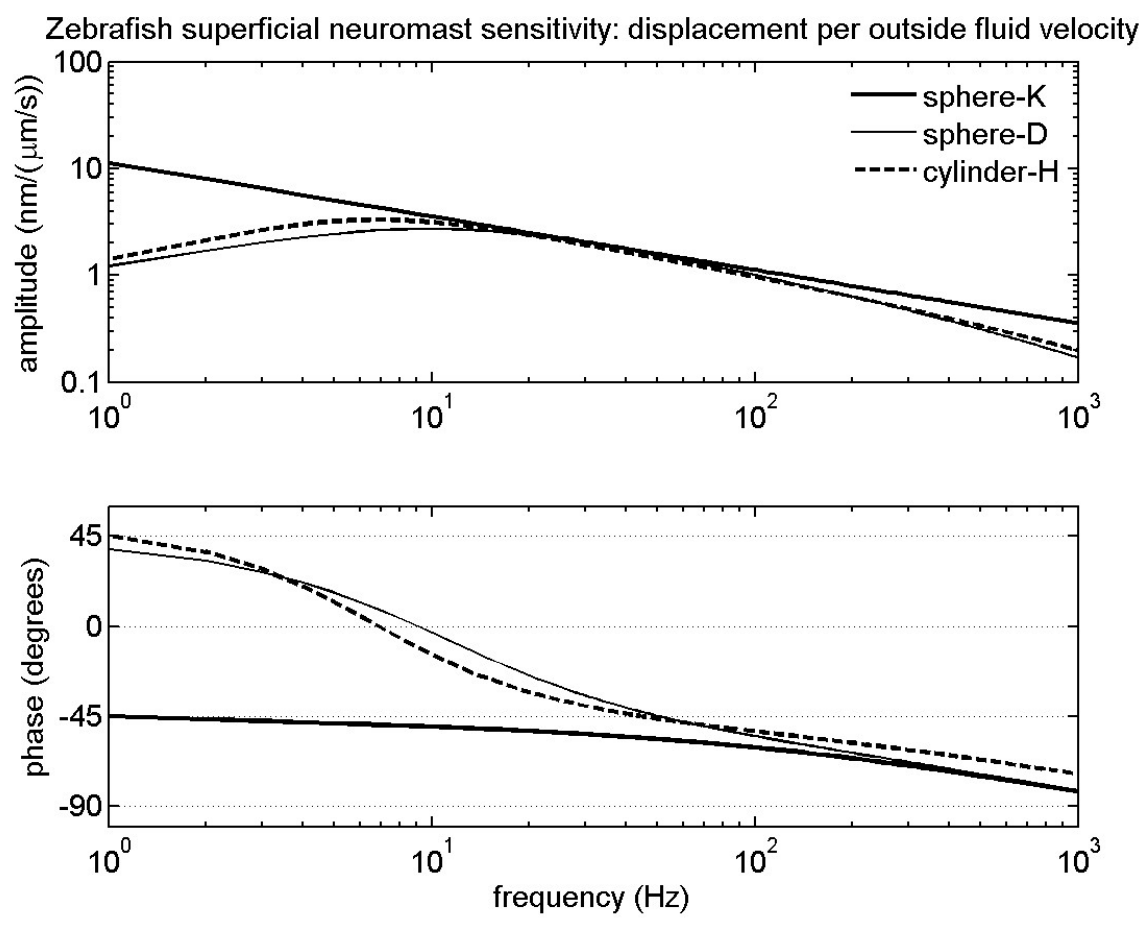

Figure 3-14: Cupular sensitivity of the superficial neuromast system. The neuromast displacement per outside water volume velocity is compared for the models of KKalmijn (1988), D-Dinklo (2005), and H-Humphrey et al. (2001), using parameters specific to the larval zebrafish. The cupula radius and length are $5 \mu \mathrm{m}$ and 34 $\mu \mathrm{m}$, respectively. The density of the gelatinous cupula is taken to be equal to that of the fluid around it, $1000 \mathrm{~kg} / \mathrm{m}^{3}$, and the viscosity of the fluid is $0.001 \mathrm{~kg} / \mathrm{m}$ $\mathrm{s}$. The boundary layer velocity profile is for a uniformly oscillating flow above a flat surface (see equation 3.9). For Kalmijn's model (using spherical drag), $K=$ $6.4 \mu \mathrm{N} / \mathrm{m}$ and $B=32.6 \mu \mathrm{Ns} / \mathrm{m}$. For Dinklo's spherical model, $f_{t}=6336 \mathrm{~Hz}$ and $N_{r}=0.0017$. For Humphrey et al.'s cylindrical model, $S=2.1 \times 10^{-15} \mathrm{Nm} / \mathrm{rad}$ and $R=1 \times 10^{-17} \mathrm{Nms} / \mathrm{rad}$. See text for details about parameter values. Note the scaling differences in the units of amplitude. 
sensitive in the lowest frequencies, and lags the outside water velocity by 45 degrees at low frequencies and closer to 90 degrees at higher frequencies. This indicates that the neuromast is acting somewhere between a velocity detector and displacement detector. Dinklo's model and Humphrey et al.'s model show that the neuromast is most sensitive to velocity around $11 \mathrm{~Hz}$. This is also the cutoff frequency, after which there is a decline of $10 \mathrm{~dB} / \mathrm{dec}$ (Dinklo, 2005). The phase indicates that at low frequencies, the cupular displacement leads the outside velocity by about 45 degrees, or lags the outside acceleration by 45 degrees. This was Kalmijn's (1988) assertion of how the phase should behave for the superficial neuromasts, as the geometric mean of the outside water velocity and acceleration. At higher frequencies, the phase indicates that it detects a combination of the outside water velocity and displacement.

\subsubsection{Models for the Mottled Sculpin}

The canal neuromast models (Kalmijn and van Netten), and the superficial neuromast models (Dinklo and Humphrey et al.), use slight variations on a driven and damped harmonic oscillator model. The most complete model is Humphrey et al.'s model (equation 3.10, who integrates the boundary layer velocity profile over the entire neuromast, rather than using just one point in the boundary layer to represent the velocity. However, as was seen in figure 3-14, even the models which just use one point in the boundary layer have practically the same results. In addition, neuromast shape does not seem to make a big difference when volumes are either similar or conserved (i.e. the spherical vs. cylindrical superficial neuromast of figure 3-14). Size of the neuromast does matter, as can be seen in the changes of resonance for canal neuromasts of figure 3-6.

The generalized equation for the oscillating neuromast system in a moving fluid 


$$
\begin{aligned}
& \int_{0}^{L}\left[M_{\text {Nmast }} \frac{d^{2} x}{d t^{2}}\right] d y= \\
& \quad \int_{0}^{L}\left[-K x-D \frac{d(x-w)}{d t}-M_{\text {add }} \frac{d^{2}(x-w)}{d t^{2}}-M_{\text {vis }} \frac{d^{2}(x-w)}{d t^{2}}+\rho \forall \frac{d^{2} w}{d t^{2}}\right] d y,
\end{aligned}
$$

where all variables have a dependency on the height above the surface, $y$, and some variables have a time dependency, $x$ is the displacement of the neuromast, $w$ is displacement of the local fluid, $K$ is the restoring force (i.e. sliding or coupling stiffness) per unit displacement, $D$ is the drag force per unit velocity that the fluid exerts on the neuromast, $M_{\text {Nmast }}$ is the mass of the neuromast, $M_{\text {add }}$ is the added mass from potential flow theory, which is due to accelerating fluid with the neuromast, $M_{\mathrm{vis}}$ is the equivalent inertia due to rotational and nonlinear effects introduced by the boundary layer around the neuromast, and $\rho \forall$ is the mass of water of equivalent volume to the neuromast (Brennan, 1982; Pan and Chew, 2002). That final term is a buoyancy force, due to the pressure work that is needed to oscillate the entire fluid, and although independent of the neuromast motion, still does work on it. Only Humphrey et al.'s (1993) model comes closest to including all of these terms, seemingly neglecting only the final buoyancy term.

Since the models that represented the velocity of the boundary layer at one point compared well with those that integrated the boundary layer velocity profile along the neuromast, adopting the former system simplifies matters considerably. Equation 3.11 can be recast in steady state transfer function form representing the cupular sensitivity as

$$
\frac{x(\omega)}{u}=\frac{D+i \omega\left(M_{\mathrm{Nmast}}+M_{\mathrm{add}}+M_{\mathrm{vis}}\right)}{K+i \omega D-\omega^{2}\left(\rho \forall+M_{\mathrm{add}}+M_{\mathrm{vis}}\right)},
$$

where $D$ and $M_{\text {vis }}$ can be functions of $\omega$.

To develop a model for the canal neuromast of the Mottled Sculpin, the frequency dependent drag for a flattened elliptic cylinder would be needed. As the elliptic cylin- 
der is a less important topic than the cylinder for research, there are comparatively few studies available. Experimental studies of the drag on an elliptical cylinder include Richards (1934), Badr (1994b), Bahr and Kocabiyik (1997), Gos'kov et al.(1998), Nair and Sengupta (1997), and others, but often the flow has shedding, indicating amplitudes greater than what the neuromast would experience. An analytical solution for oscillating inviscid flow past an elliptical cylinder was given by Badr (1994a), but for the neuromast, viscous forces will be to significant to ignore.

Since volume conserved shapes seem to give comparable results, a spherical, cylindrical, and finite sized plate model will be compared. Stokes (1851) calculated the drag on an oscillating sphere (equation 3.6), on a cylinder (Appendix A), and on a flat plate, which is given as (Lamb, 1932)

$$
D=\mu \frac{U_{0} A(1+i)}{\delta(\omega)} e^{i \omega t}
$$

where $U_{0}$ is the velocity amplitude of the oscillating plate, $\mathrm{A}$ is the area of one side of the plate, $\mu$ is the viscosity of the fluid, $\omega$ is the angular frequency of oscillation, and where $\delta(\omega)$ was previously defined as an approximate measure of the boundary layer thickness (equation 2.2). For all models, the velocity profile for oscillating flow in a pipe will be used (Schlichting, 1979), and its relation to the outside water volume acceleration is given by equation 3.7 .

Assuming that the cupula is of a similar density to water, the mass of the canal neuromast, $M_{\text {Nmast }}$, and the mass of the water volume displaced, $\rho \forall$, are equal. Approximating the cupula of the canal neuromast as an elliptical cylinder, the mass is $\rho \pi a b h=\pi\left(1000 \mathrm{~kg} / \mathrm{m}^{3}\right)(200 \mu \mathrm{m})(25 \mu \mathrm{m})(1 \mathrm{~mm})=1.6 \times 10^{-8} \mathrm{~kg}$. The added mass for an ellipse is $\pi a b$, for a sphere is $(2 / 3) \pi r^{3}$, and for a cylinder is $\pi r^{2}$, where $a$ and $b$ are the radii of the major and minor axes of the ellipse, and $r$ is the effective radius which will allow the volume of the ellipse to be conserved for either the sphere or the cylinder. $M_{\text {vis }}$ is only known for the cylinder, having been expressly calculated by Humphrey et al. (1993), and appearing in equation 3.10. The flat plate model will use the added mass of the ellipse, but twice (two sides) the drag of a flat plat given 
in equation 3.13 .

Figure 3-15 compares the results of modeling the canal neuromast as either a sphere, cylinder, or a flat plate with the front being approximated as an elliptic cylinder. The model parameters were determined by fitting the plate model results to the empirical results of Coombs and Janssen (1990) in the frequency range of 10$100 \mathrm{~Hz}$. The cylinder model shows a much greater system sensitivity, being on the order of 5 times greater neuromast displacement per same input acceleration than the sphere or flat plate models. Though the cylinder model contains an additional inertia term, $M_{\text {vis }}$, removing it from the model does not seem to alter the results significantly in the low frequencies, but does allow for a resonance frequency to have a sharper peak at the same frequency as the sphere and flat plate models (not shown). More significant is changing the radius of the cylinder. When it is set equal to the minor axis radius, the results match those of the sphere almost identically. Both the cylinder and the plate models show that at low frequencies, the canal neuromast system becomes less sensitive (see also figure 3-17). Coombs and Janssen address this in their discussion, and think that this is a real effect. The phase of the spherical and cylinder models at low frequencies is what is expected from experimental results (Denton and Gray, 1983), but the plate model has a slightly more appropriate phase in the $10-100 \mathrm{~Hz}$ range.

The superficial neuromast model for the Mottled Sculpin has a best fit to the data of Coombs and Janssen (1990) when the parameters are the same as those for the zebrafish (see figure 3-14). This is not terribly surprising, as superficial neuromasts are often similar across species.

\subsection{Thresholds of detection and saturation}

With analytical models available to describe canal and superficial neuromast motion, the next question to address is minimum input levels of fluid motion which result in detection, or conversely maximum input levels of fluid motion which result in saturation (and loss of information). This section will detail the process of determining 

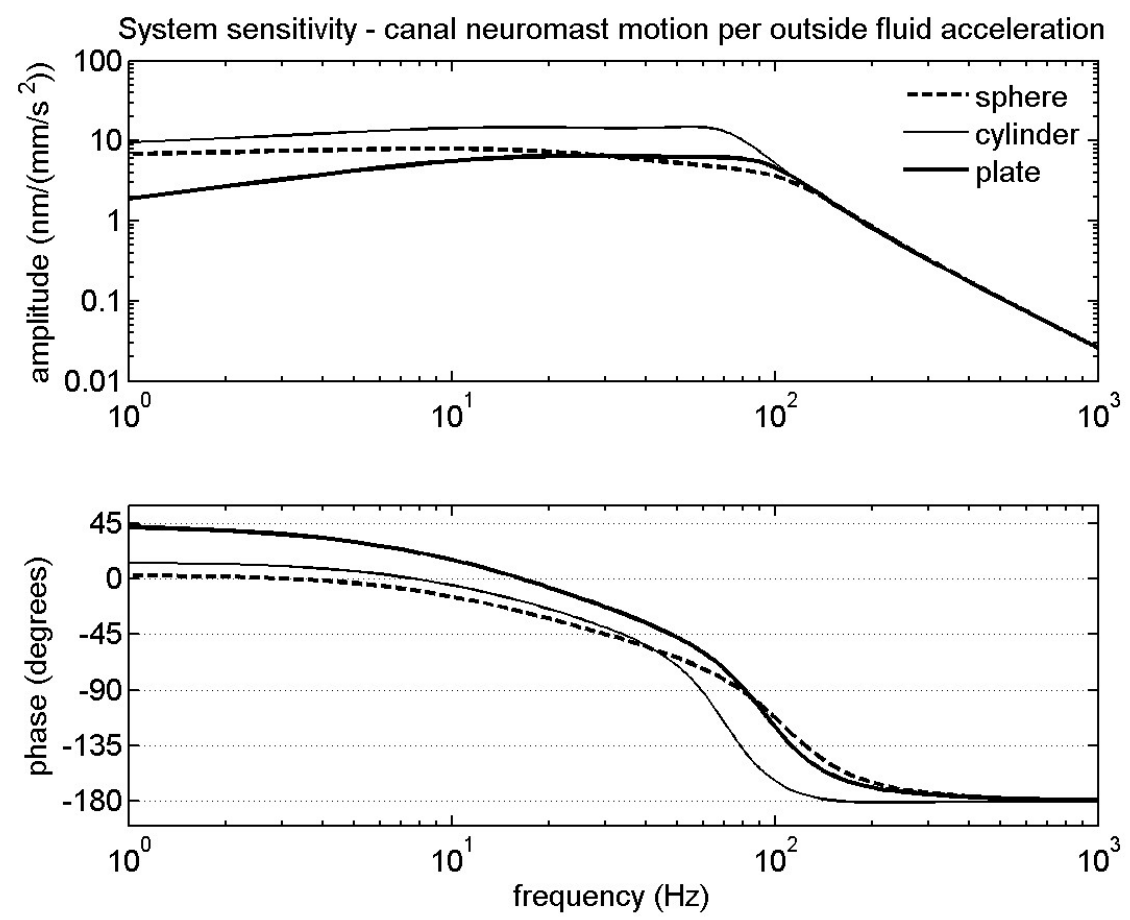

Figure 3-15: Cupular sensitivity of the canal neuromast system for the Mottled Sculpin, using a second order model. The neuromast displacement per outside water volume acceleration is compared for a spherical, cylindrical, and flat plate model of the canal neuromast. The dimensions of the keel shaped cupula of the canal neuromast are assigned values of $(400 \mu \mathrm{m}) \times(50 \mu \mathrm{m}) \times(1 \mathrm{~mm})$, and represent the diameter of the major, minor, and vertical axes of an elliptical cylinder. The sphere and cylinder their radius adjusted so as to have volumes equal to that of the elliptical cylinder. The spherical model uses the model of van Netten (2006), and has a sliding stiffness of $K=0.03 \mathrm{~N} / \mathrm{m}$, a transition frequency of $f_{t}=33 \mathrm{~Hz}$, and a resonance number of $N_{r}=10$ (see text). The cylinder model uses the drag and added mass model of Humphrey et al. (2001), and has values determined in Appendix A. The flat plate model uses the drag on its two dominant sides, and the front is modeled like an elliptical cylinder. The cupula is assigned a density equal to that of the surrounding fluid, $1000 \mathrm{~kg} / \mathrm{m}^{3}$, the subdermal canal is assigned a diameter of $1 \mathrm{~mm}$, and the canal fluid is assigned a viscosity of $0.005 \mathrm{~kg} / \mathrm{m}-\mathrm{s}$. Note the scaling differences in the units of amplitude. 
the threshold of detection to water motion incident upon the neuromasts. A distinction is made between still water and background flow environments, because there is some evidence that the fish processes the information in a different manor for each environment (Kanter and Coombs, 2003; Chagnaud et al., 2006).

\subsubsection{Mechanical definition using neuromast displacement}

There is a minimum displacement needed by the neuromast to overcome random motions it may experience by being immersed in a fluid. The neuromast displacement threshold is based on stochastic and mechanical properties of the hair cell and overlying cupula. The hair cell transducer apparatus has a random noise component based on the thermal noise energy and the molecular gating force, which translates into an uncertainty of $6.5 \mathrm{~nm}$ (Dinklo, 2005; van Netten, 2006). Ensemble averaging will reduce this number by the square root of the number of hair cells present in the superficial neuromast, since each hair cell will act as an independent detector of the cupular displacement. Secondly, the Brownian motion of the fluid around the cupula leads to a displacement uncertainty inversely proportional to the square root of the coupling stiffness, $K$. Dinklo (2005) and van Netten (2006) give this displacement uncertainty equal to $\sqrt{k T / K}$, where $k$ is Boltzmann's constant, and $T$ is the absolute temperature.

To find a corresponding velocity detection threshold, van Netten (2006) divides the neuromast displacement threshold by its cupular sensitivity at $0 \mathrm{~Hz}$. The cupular sensitivity is defined as displacement per fluid velocity input, and has units of ms. For the ruffe, its canal neuromasts have on the order of 1000 hair cells each, and so the transducer displacement noise is $6.5 \mathrm{~nm} / \sqrt{1000} \approx 0.2 \mathrm{~nm}$. With a sliding stiffness of $K=0.13 \mathrm{~N} / \mathrm{m}$, and with $k T \approx 4.1 \times 10^{-21} \mathrm{~J}$, the displacement noise due to Brownian motion is $0.2 \mathrm{~nm}$. The neuromast displacement threshold is the root-mean-square of the two, or about $0.3 \mathrm{~nm}$. van Netten points out that behavioral results from experiments by Kuiper (1956) indicate the displacement threshold to be about 2.5 nm. Dividing this number by a cupular sensitivity of $0.23 \mathrm{~ms}$, the velocity detection threshold for the supraorbital canal neuromast is in the range of $1-10 \mu \mathrm{m} / \mathrm{s}$. 
The acceleration detection threshold is found by dividing the velocity detection threshold by the canal sensitivity. van Netten (2006) determined that for the supraorbital canal neuromasts of the ruffe, a detection threshold to the outside water volume acceleration was on the order of $0.1-1 \mathrm{~mm} / \mathrm{s}^{2}$. The values given for the acceleration threshold of the canal neuromasts were obtained through the combination of nanometer accuracy measurements of the cupular- and canal fluid dynamics (Curcic-Blake and van Netten, 2005), along with analytical modeling. Although individual canal neuromasts show increased sensitivity at certain frequencies, the combined effect of the canal and boundary layer filtering effects results in an almost unform sensitivity to acceleration from 1-100 Hz. (van Netten, 2006). The magnitude and uniformity of the acceleration threshold in this frequency range has been validated in behavioral experiments (Coombs and Janssen, 1990), though beyond this frequency range the acceleration threshold worsens.

There has been some difficulty in defining the velocity detection threshold for superficial neuromasts. Kroese et al. (1978) determined the superficial neuromasts of the Xenopus Laevis to have a velocity detection threshold of $38 \mu \mathrm{m} / \mathrm{s}$. They used potential flow theory for an oscillating sphere next to a wall to calculate the hair displacement, but did not take into account the effects of the boundary layer. The hair cells were buried within the boundary layer, experiencing a reduction in displacement compared to their calculations. Gorner (1963) determined a velocity detection threshold of $25 \mu \mathrm{m} / \mathrm{s}$ for Xenopus Laevis. In their case, constant laminar flow stimulation was used, which means the boundary layer profile is dependent on the distance of the hair cell from the source of the flow on the body, and the length of time elapsed between the start of the flow and the measured response.

The presence of a boundary layer has complicated the process of determining the velocity threshold for superficial neuromasts. If there were a way to determine the boundary layer velocity profile at detection, the velocity threshold could be defined as the (modeled) minimum fluid velocity at the tip of the cupula needed to reach the displacement threshold. Dinklo (2005) created an oscillating boundary layer over the surface of a larval zebrafish using a mechanically controlled micropipette, thereby 
controlling the boundary layer. He then adapted van Netten's theoretical work on canal neuromasts for superficial neuromasts by using an oscillating surface boundary layer model developed by Stokes (1851).

The model (see figure 3-14) essentially defined a tip-displacement to outside fluid velocity sensitivity. The cupular sensitivity is a mechanical property, which Dinklo determined by fitting an analytical model to experimental data. He did not go so far as to determine a velocity detection threshold, but this can be determined using his available data. Using van Netten's (2006) definition of velocity detection threshold, which equals the neuromast displacement detection threshold divided by cupular sensitivity, the outside water volume velocity detection threshold for the zebrafish superficial neuromast based on Dinklo's research would be $25 \mathrm{~nm} / 1.2 \mathrm{~ms}=21 \mu \mathrm{m} / \mathrm{s}$ at $0 \mathrm{~Hz}$.

Superficial neuromasts exposure to the outside water volume motion is mediated by the boundary layer. When the boundary layer dynamics are undetermined, it is valuable to look at the dynamics of the superficial neuromast to any water motion not just its response to fluid velocity. Secondly, it is valuable to extend the definition of detection threshold to all frequencies. Therefore for superficial neuromasts, a definition of detection threshold to general water motion, over all frequencies, could be

$$
\text { detection threshold }=\frac{\text { neuromast displacement detection threshold }}{(\text { cupular or system sensitivity }) \omega^{n}},
$$

where $n$ is $[-1,-0.5,0,0.5,1]$. For local water motion, the cupular sensitivity would be used, whereas when the outside water volume motion is desired, the system sensitivity would be used (which is just the cupular sensitivity multiplied by the boundary layer transfer function). Since the neuromast displacement detection threshold has units of meters, the cupular and system sensitivity has units of seconds, and the angular frequency, $\omega$, has units of radians $/ \mathrm{Hz}$, combinations of all three can lead to many detection motions. For example, when $n$ is $-1,0$, and 1, acceleration, velocity, and displacement motions are detected, respectively. When $n=-0.5$, the water motion 
is the geometric mean of velocity and acceleration. When $n=0.5$, the water motion detected is the geometric mean of velocity and displacement.

Figure 3-16 shows the results of the larval zebrafish detection threshold to various types of water motion. In particular, the detection threshold becomes independent of frequency for certain ranges and certain types of water motion. In the top plot, the detection threshold is approximately constant for local water velocity motions up to about $10 \mathrm{~Hz}$ (mean approx. $2 \mu \mathrm{m} / \mathrm{s}$ ), and then it transitions to detecting local water displacements (mean approx $25 \mathrm{~nm}$ ). In the bottom plot, the detection threshold is approximately constant to the geometric mean of the outside water velocity and acceleration up to about $6 \mathrm{~Hz}$ (mean approx. $2 \sqrt{(\mu \mathrm{m} / \mathrm{s})\left(\mathrm{mm} / \mathrm{s}^{2}\right)}$ ), then is approximately constant to the outside water velocity up to about $20 \mathrm{~Hz}$ (mean approx. 10 $\mu \mathrm{m} / \mathrm{s}$ ), and then is approximately constant to the geometric mean of the outside water velocity and displacement up to about $100 \mathrm{~Hz}$ (mean approx. $30 \sqrt{(\mu \mathrm{m} / \mathrm{s})(\mathrm{nm})}$ ). Beyond $200 \mathrm{~Hz}$, the detection threshold transitions to detecting the outside water volume displacement (mean approx. $27 \mathrm{~nm}$ ). The introduction of boundary layer dynamics significantly increases the complexity of the superficial neuromast motion and what outside water motion it detects.

The problem with an empirical neuromast displacement detection threshold is that the location of the measurement must be accounted for. This location is almost always the tip of the cupula, but both Dinklo (2005) and McHenry and van Netten (2008) found that the flexible tip of the cupula bends more than the stiffer base region, and so displacement at the tip does not necessarily translate into displacement at the level of the hair tips (cilia). Dinklo estimated a 20-30 \% reduction for hair tip displacement as compared with that measured half way up the cupula.

A way to standardize results across experiments would be to divide the neuromast displacement detection threshold by the height at which the models fit the data. For small displacements relative to the length of the cupula (i.e. nanometer displacements for micrometer length cupula) so that arc length can be approximated by displacement, a critical angle, or neuromast deflection threshold can be defined. Since the models used to fit the data are linear, 2nd order systems, the results will average the 

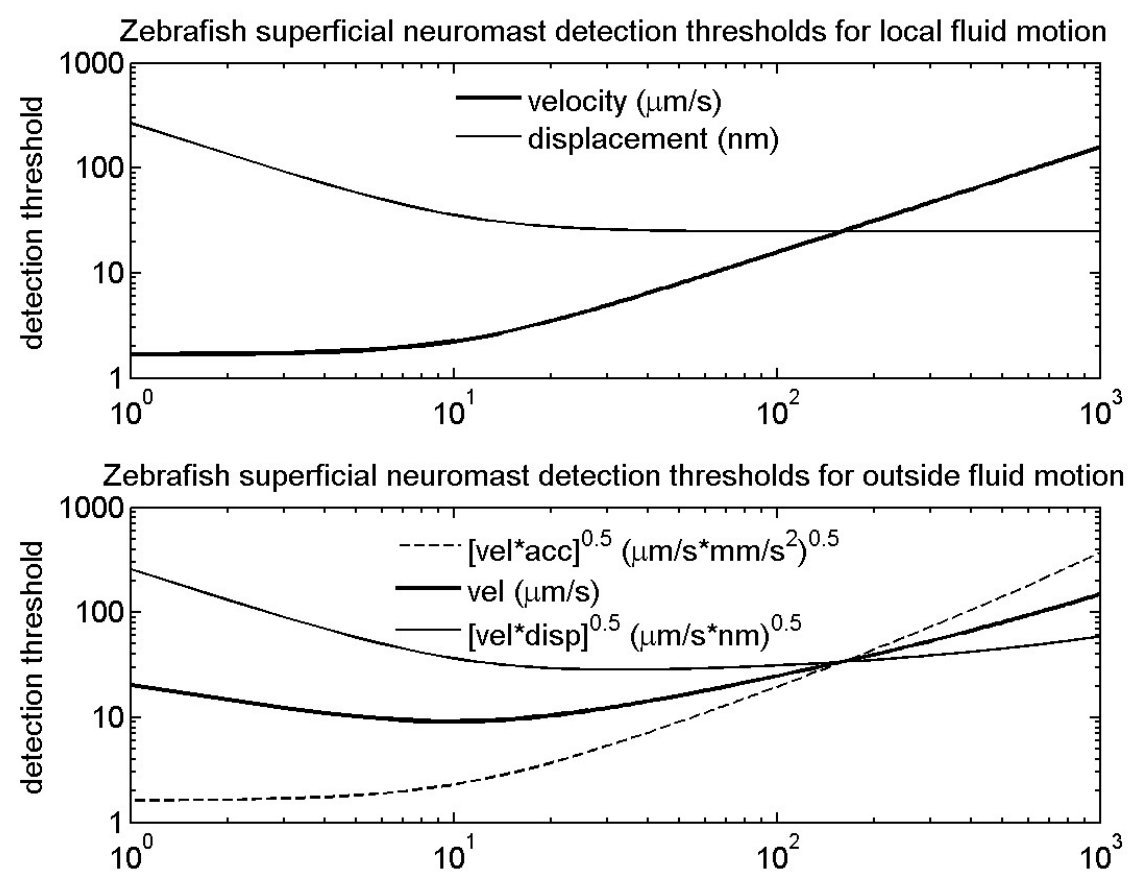

Figure 3-16: Detection thresholds for the larval zebrafish superficial neuromasts. The detection threshold is defined as the minimum motion of either the local or outside water volume which will cause the superficial neuromast to rotate past a critical angle (or equivalently, have the tip displaced a critical distance). The critical angle (or critical displacement distance) is the motion of the superficial neuromast beyond any random motions it may generally experience (see text). The top plot shows the detection threshold to either local velocity or displacement motions. The bottom plot shows the detection threshold to water motions outside of the boundary layer. Note that over certain frequency ranges, the detection threshold will be approximately constant to a type of water motion. The range of water motions include the typical acceleration, velocity, and displacement motions, and also the geometric mean of velocity and acceleration, and the geometric mean of velocity and displacement. The results presented are for the larval zebrafish, and are based on extensions of the theory and work of Dinklo (2005) and van Netten (2006). 
real motions of a flexible cupular, and the critical angle will represent those averaged results.

Normalizing by the model fit cupula length also presents another way to interpret the cupular sensitivity. When the tip displacement is separated into a pivot angle times the cupula length ( $x=\theta L$ ), the cupular sensitivity, $S v$, can be rewritten as an angle to strain rate sensitivity:

$$
S v=\frac{\text { tip displacement }}{\text { fluid velocity at tip }}=\frac{x}{u}=\frac{\theta L}{u}=\frac{\theta}{S}
$$

Here, $S$ is an approximate strain rate threshold, defined as the tip-to-base velocity difference $(u-0)$ divided by the cupula length, $L$. The physical meaning of this is that the hair pivots through an angle in proportion to the approximate strain rate of the fluid. The approximate strain rate detection threshold would be found by dividing the velocity detection threshold values (see figure 3-16) by the length of the cupula. This may prove useful in comparing detection thresholds across species or experiments, especially when information about the neuromast displacement detection threshold is absent. Examples that illustrate this will appear later in the thesis.

For the superficial neuromasts of the larval zebrafish, which have approximately 10 hair cells per neuromast, the combined displacement uncertainty is $(25 \mathrm{~nm}+$ $6.5 \mathrm{~nm} / \sqrt{10})^{0.5} \approx 25 \mathrm{~nm}$. An approximate deflection threshold for the larval zebrafish would be equal to the displacement threshold divided by the cupula length, $\theta=$ $25 \mathrm{~nm} / 34 \mu \mathrm{m}=7 \times 10^{-4} \mathrm{rad}$. Using the outer water volume velocity threshold in figure $3-16$, and assuming a cupula length of $34 \mu \mathrm{m}$, the approximate strain rate detection threshold would be $S=[0.60,0.27,0.48,0.73] s^{-1}$, at frequencies $f=\left[\begin{array}{llll}1 & 10 & 50 & 100\end{array}\right]$ $\mathrm{Hz}$. There is a minimum strain rate around $10 \mathrm{~Hz}$, where the superficial neuromast appears most sensitive.

As mentioned at the beginning of this section, determining the saturation threshold is basically an identical process to determining the detection thresholds. Now, instead of a neuromast displacement detection threshold, there is a general neuromast displacement distance of about $100 \mathrm{~nm}$ (Kroese and van Netten, 1989), beyond 
which the hair cells are saturated. The next section will utilize this information when determining detection and saturation thresholds for the Mottled Sculpin, as used in this thesis.

\subsubsection{Thresholds of detection and saturation for the Mottled Sculpin in still water enviroments}

To determine the detection threshold levels for the Mottled Sculpin, the neuromast displacement threshold is needed. For the canal neuromast of the ruffe, van Netten (2006) showed that the neuromast displacement threshold is about $0.3 \mathrm{~nm}$ on empirical and theoretical grounds, and upwards of $2.5 \mathrm{~nm}$ based on behavioral results. The lower bound value is based on the stochastic properties of the hair cell transducer apparatus, and on the Brownian motion of cupula. The transducer displacement uncertainty is $6.5 \mathrm{~nm}$ for each hair cell, but is reduced by the square root of the number of hair cells in the neuromast due to ensemble averaging. The displacement uncertainty due to the Brownian motion is inversely proportional to the square root of the sliding stiffness. The stiffer the material, the less random motions have an effect, and consequently the easier it is for the neuromast to detect movements greater than the noise level.

Without direct measurement of the sliding stiffness for the canal neuromast of the Mottled Sculpin, it is difficult to gage the accuracy of the models presented for the Mottled Sculpin lateral line system. At best, they can serve as a starting point for further investigation. Coombs and Janssen (1990) conducted behavioral and neurophysiological studies on the threshold of detection for the Mottled Sculpin. Figure 3-17 shows the results of fitting the flat plate model to their empirical results for the canal lateral line system. In tuning the flat plate model to the data, increasing the sliding stiffness, $K$, tended to lower the cutoff frequency, and increasing $K$ increased the cutoff frequency. There is a trade off, for decreasing $K$ also increased sensitivity (lowered the threshold). Therefore, there is a cupular sensitivity-frequency cutoff balance, which van Netten (2006) gives as $S_{v} \cdot f_{c}=(2 \pi)^{-1}$. Secondly, the height 
of the canal (and therefore the approximate height of the canal neuromast) had a significant effect on the lower frequency curve. Increasing the canal diameter greatly increased sensitivity, and decreasing canal diameter. This is in keeping with earlier results (see figure 3-10), which showed that increasing the canal diameter relative to the boundary layer significantly increased the area of flow in the pipe that would not experience any reduction in velocity. In determining the displacement noise, the number of hairs cells had the effect of raising or lowering the detection threshold level. To match the detection threshold determined by the behavioral results where Coombs and Janssen placed the stimulus near the Sculpin head, $K=0.03 \mathrm{~N} / \mathrm{m}$, the canal diameter is $1 \mathrm{~mm}$, and the neuromast has 20 hair cells. According to anatomical studies by Janssen et al. (1987), all of the subdermal canals narrowed in the region of the canal neuromast, and the infraorbital canal in particular had a diameter of 2.5-4 $\mathrm{mm}$ in that narrow region. The study also seems to indicate that there are a small number of hair cells. There is nothing said about the coupling stiffness, but for the amount of cupula that sits above the tiny sensory strip, it is conceivable that it would be lower than what was measured in the ruffe $(0.1 \mathrm{~N} / \mathrm{m}$, van Netten, 2006). Finally, saturation threshold is calculated using equation 3.14, with the neuromast displacement set to $100 \mathrm{~nm}$ (Mogdans et al., 2003).

Figure 3-17 also compares how the spherical and cylindrical neuromast models compare when using the parameters determined by the flat plate neuromast model. Both models use the empirically determined sliding stiffness, $K=0.03 \mathrm{~N} / \mathrm{m}$, and have their radius adjusted so as to have their volumes equal the elliptical volume of the canal neuromast used in the flat plate model. Both models have lower detection thresholds in the lower frequency range relative to the flat plate model. In the lowest frequency range $(1-10 \mathrm{~Hz})$, the cylindrical model decreases in sensitivity like the flat plate model, whereas the spherical model stays constant. Essentially, what this means is different models provide different insights into the mechanics of what is happening inside the canal filtering system, and that each model must be specifically tuned to match the data.

There is one very significant difference between the models and the experimental 


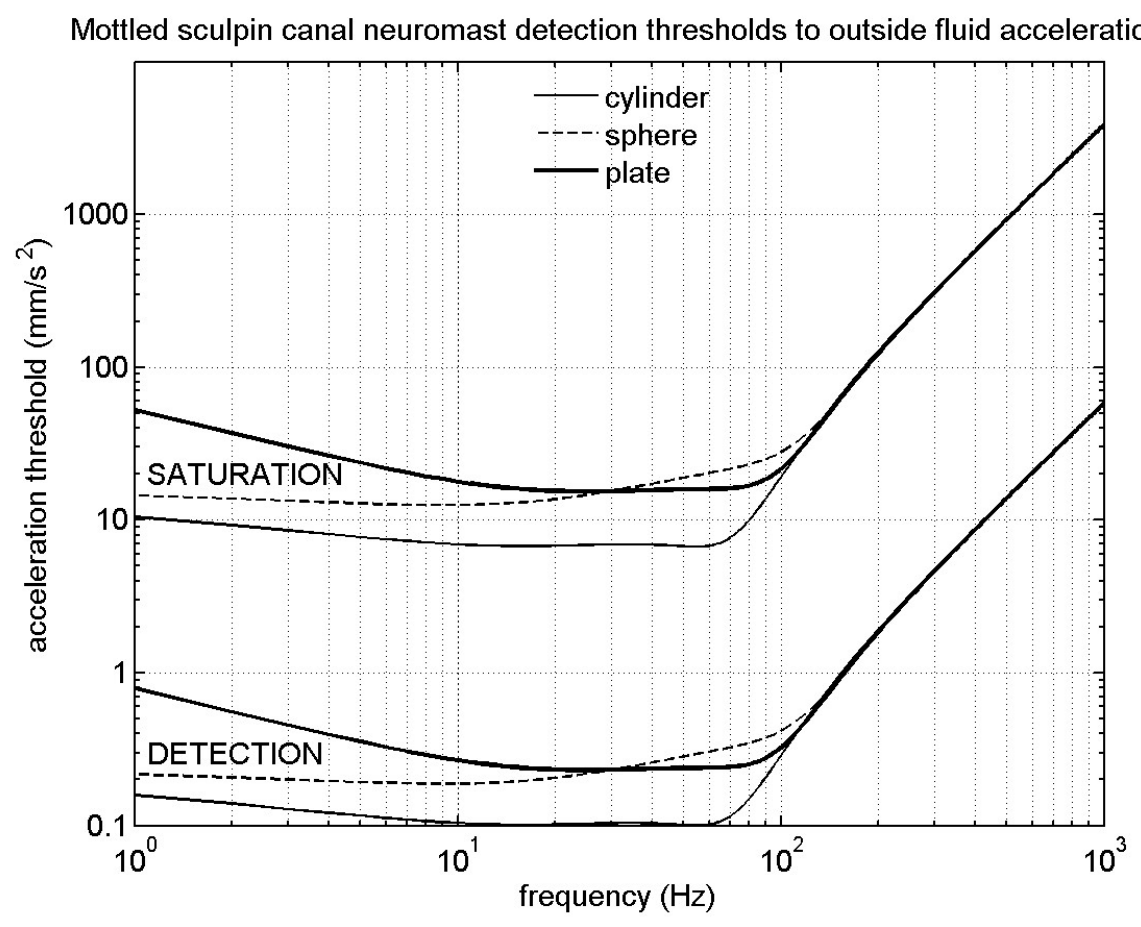

Figure 3-17: Cupular sensitivity of the canal neuromast system for the Mottled Sculpin, using a second order model. The neuromast displacement per outside water volume acceleration is compared for a spherical, cylindrical, and flat plate model of the canal neuromast. The dimensions of the keel shaped cupula of the canal neuromast are assigned values of $(400 \mu \mathrm{m}) \times(50 \mu \mathrm{m}) \times(1 \mathrm{~mm})$, and represent the diameter of the major, minor, and vertical axes of an elliptical cylinder. The sphere and cylinder their radius adjusted so as to have volumes equal to that of the elliptical cylinder. The spherical model uses the model of van Netten (2006), and has a sliding stiffness of $K=0.03 \mathrm{~N} / \mathrm{m}$, a transition frequency of $f_{t}=33 \mathrm{~Hz}$, and a resonance number of $N_{r}=10$ (see text). The cylinder model uses the drag and added mass model of Humphrey et al. (2001), and has values determined in Appendix A. The flat plate model uses the drag on its two dominant sides, and the front is modeled like an elliptical cylinder. The cupula is assigned a density equal to that of the surrounding fluid, $1000 \mathrm{~kg} / \mathrm{m}^{3}$, the subdermal canal is assigned a diameter of $1 \mathrm{~mm}$, and the canal fluid is assigned a viscosity of $0.005 \mathrm{~kg} / \mathrm{m}-\mathrm{s}$. 
results. The behavioral results indicate that above $150 \mathrm{~Hz}$, the sensitivity declines at a very fast rate, upwards of $120 \mathrm{~dB}$ per decade. This is not uncommon, and has been observed in the saccular hair cells of Opsanus and goldfish (Popper and Fay, 1999; Lewis 1992). Essentially, this is equivalent to adding a sixth order component to the model, $-M_{m}\left(\partial^{6} x / \partial t^{6}\right)$ to the right hand side of equation 3.11 , or $+M_{m}(i \omega)^{6}$ to the denominator of equation 3.12. Figure 3-18 shows the result when $M_{m}=3 \times 20^{-20} \mathrm{~kg} \cdot \mathrm{s}^{4}$ for the head canal lateral line. The fact that this term needs to be added indicates that a second order model does not capture well enough what happens at high frequency for the canal lateral line system of the Mottled Sculpin. Either there are material changes at high frequencies, or the shape of the canal serves to limit the overall sensitivity. Further research is needed to determine the answer to this.

Figure 3-18 also shows the results for specifically tuning the spherical and cylindrical neuromast models to data of Coombs and Janssen (1990). Each model was matched to a location on the sculpin based on shapes of the threshold curve models of figure 3-17 matching the tuning curves of figure 3-18. There was a small allowable range of the sliding stiffness, $K=0.01-0.03 \mathrm{~N} / \mathrm{m}$ that allowed for proper placement of the cutoff frequency. Similarly, changing the canal diameter significantly affected the shape of the curves in the low frequency range $(<10 \mathrm{~Hz})$, so that the model fits prescribed certain diameters for each model. The sixth order component had a small range of values, $M_{m}=(1-3) \times 20^{-20} \mathrm{~kg} \cdot \mathrm{s}^{4}$. Finally, the number of hair cells in the neuromast affected the threshold levels. Once the other parameters were set, the shapes of the curves were determined, and number of hair cells allowed the final fitting of the models to the data. However, the values are clearly suspect for spherical and cylindrical neuromast models, being 1 hair cell and 0.25 hair cells, respectively. Obviously this indicates a problem with the models. However, this is another possible explanation. Coombs and Janssen (1990) postulate that the differences in detection threshold levels at the head, trunk, and tail, were due to differences in density, size, and shape of neuromasts in each location. They rule out the possibility that only the head was actually detecting the oscillating dipole signal, and that moving it towards the tail would decrease the signal strength to the head. However, what they did not 
address was the fact that the direction that the spherical stimulus was vibrating, in the vertical (dorsal-ventral) direction, was exactly tuned to maximally stimulate the canal lateral line on the head. The trunk and tail lateral line canals lie perpendicular to the axis of oscillation, and would only minimally be stimulated. The results of this stimulation are shown in the first 3D chapter. If the sphere had been vibrating parallel to the trunk and tail lateral line canals (as is the case for many subsequent experiments), the threshold curves would likely be changed, showing themselves to be more sensitive, in a manor similar to the head canal lateral line system. There still could be differences based on the differences in neuromast morphology, but it is likely they would be less major.

When using the definition of detection threshold (equation 3.14 to fit the superficial neuromast models to the data of Coombs and Janssen (1990), it is found that parameters of the zebrafish superficial neuromast models (figure 3-14) give the best bit to the data. Figure 3-19 shows the results of applying a noise motion level of $25 \mathrm{~nm}$ and saturation motion level of $100 \mathrm{~nm}$ to the zerbrafish superficial neuromast model. The data indicates a much larger range of variability than depicted by these superficial neuromast models, and could indicate a number of things. One possibility is that modeling the superficial neuromast as a solid cylinder or sphere is not able to adequately capture the dynamic motions. McHenry and van Netten (2007) show that the superficial neuromast has a stiff region near the base and flexible region near the tip of the cupula (see figure 3-13). However, Dinklo's (2005) data seems to suggest that these models do at least well represent the amplitude of these motions, if not the phase. Another possibility is that the local region around the superficial neuromasts are different, such as in the case of skin cell growth or scarring (Janssen et al., 1988), which would alter the local flow in a non-uniform way. Still other possibilities are that the individual hair cells associated with the neuromasts are tuned for different signals, and therefore interpret the neuromast motions in unique ways. This component would suggest differences in hair cell chemistry (i.e. amount of reserves available for replenishment), and possible feedback from the brain. 


\section{Canal neuromast detection thresholds to outside fluid acceleration for the Mottled Sculpin}

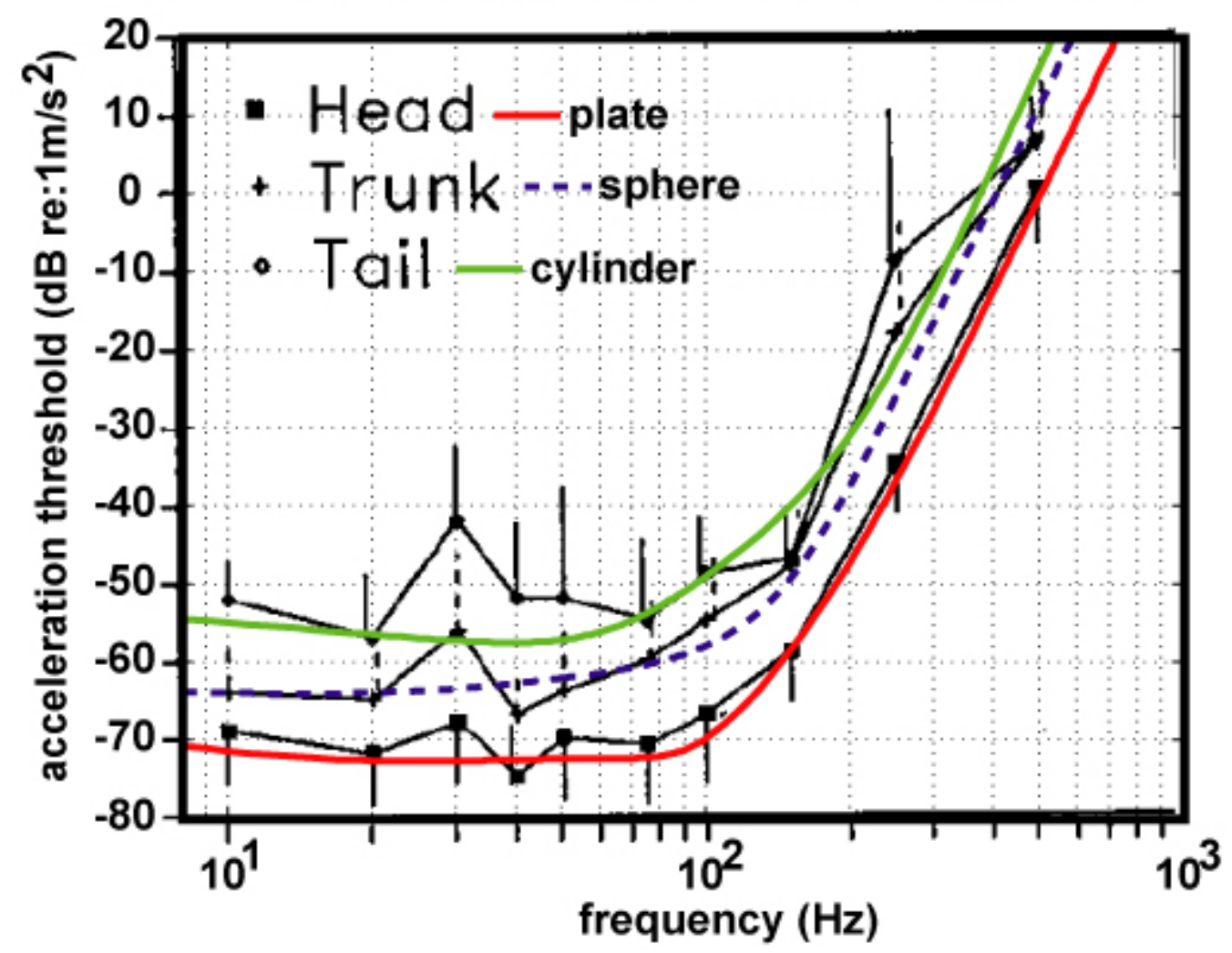

Figure 3-18: The outside acceleration threshold for the canal neuromast system is presented for modeled (colored lines) and measured (black lines) results. The data is from the behavioral experiments of Coombs and Janssen (1990), and represents the mean threshold sensitivity from 80 measurements per data point, using 4 fish. Error bars indicate the standard deviation from the mean. The neuromast is modeled as an elliptical cylinder. The height is matched to the diameter of the canal at each location. The cupula is assigned a density equal to that of the surrounding fluid, $1000 \mathrm{~kg} / \mathrm{m}^{3}$ , and the canal fluid is assigned a viscosity of $0.005 \mathrm{~kg} / \mathrm{m}-\mathrm{s}$. The model is based on equation 3.11, and pipe flow in a canal (equation 3.7), but with a sixth order component added for high frequencies $(>150 \mathrm{~Hz})$. The frequency dependent drag is specific to each type of model (see text). The values are as follows: (Head: plate model) $K$ $=0.03 \mathrm{~N} / \mathrm{m}$, canal diameter $=1 \mathrm{~mm}$, base $=(400 \mu \mathrm{m}) \times(50 \mu \mathrm{m}), M_{m}=3 \times 10^{-20} \mathrm{~kg} \cdot \mathrm{s}^{4}$, 20 hair cells; (Trunk: sphere model) ) $K=0.015 \mathrm{~N} / \mathrm{m}$, canal diameter $=0.8 \mathrm{~mm}$, base $=$ $(200 \mu \mathrm{m}) \mathrm{x}(50 \mu \mathrm{m}), f_{t}=61 \mathrm{~Hz}, N_{r}=3.6, M_{m}=3 \times 10^{-20} \mathrm{~kg} \cdot \mathrm{s}^{4}, 1$ hair cell; (Tail: cylinder model) ) $K=0.01 \mathrm{~N} / \mathrm{m}$, canal diameter $=0.6 \mathrm{~mm}$, base $=(150 \mu \mathrm{m}) \times(50 \mu \mathrm{m})$, $M_{m}=1 \times 10^{-20} \mathrm{~kg} \cdot \mathrm{s}^{4}, 0.25$ hair cells. Experimental data reproduced with kind permission from Springer Science and Business Media: Journal of Comparative Physiology A: Sensory, Neural, and Behavioral Physiology, Behavioral and neurophysiological assessement of lateral line sensitivity in the mottled sculpin, Cottus bairdi, volume 167, 1990, page 560, Coombs, S., Hastings, M., and Finneran, J., figure 3A. 


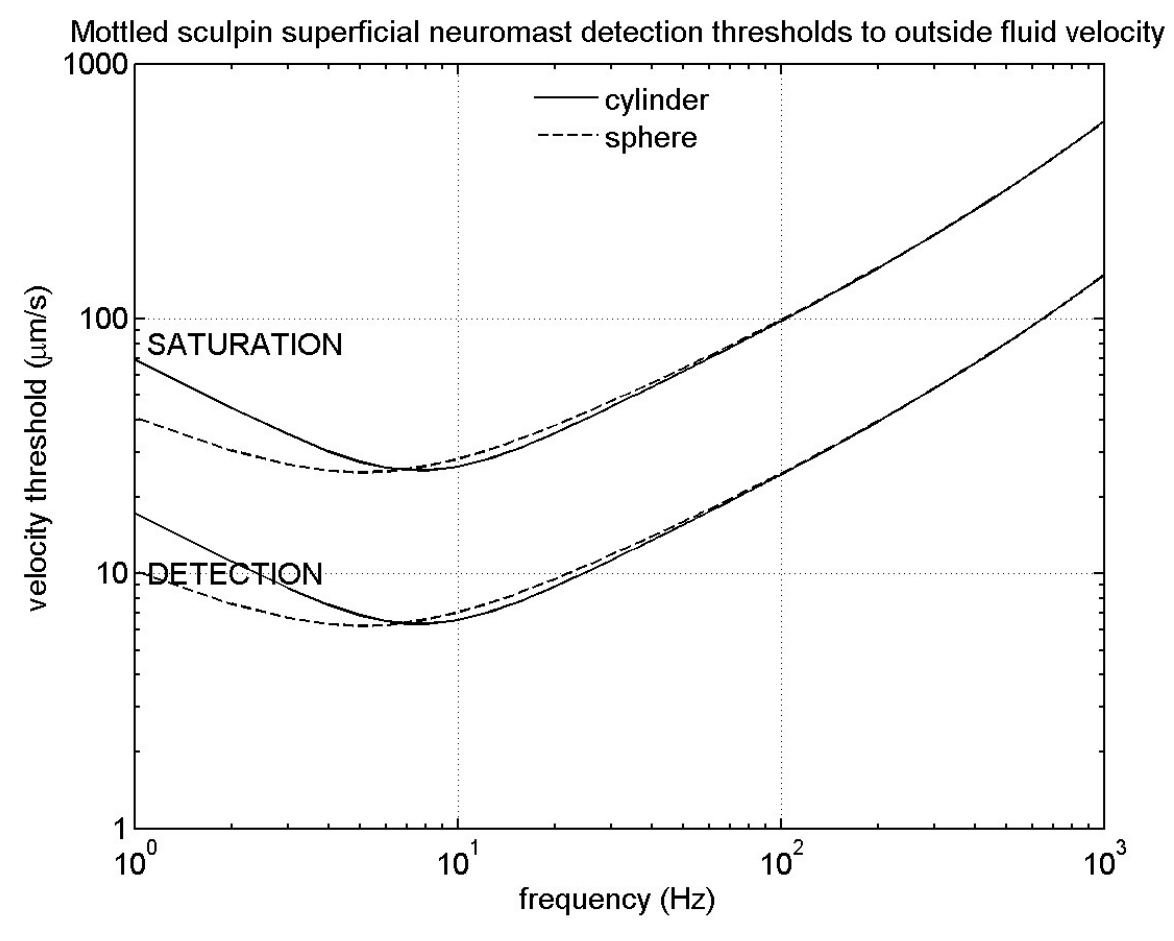

Figure 3-19: Detection and saturation thresholds for the superficial neuromasts of the Mottled Sculpin. The detection threshold is defined as the minimum motion of either the local or outside water volume which will cause the superficial neuromast to rotate past a critical angle (or equivalently, have the tip displaced a critical distance). The critical angle (or critical displacement distance) is the motion of the superficial neuromast beyond any random motions it may generally experience (see text). The saturation threshold is defined as the maximum displacement, beyond which the neuromast cannot represent the changes in motion. Actual neurophysiological data for the Mottled Sculpin shows a much wider range of slopes for the threshold of detection (Coombs and Janssen, 1990), indicating that this model may not adequately capture the real neuromast motions. However, it serves as a lower bound to the detection limits, since the experimental threshold tuning curves fall at or above this. 


\section{Chapter 4}

\section{Using CFD to calculate the stimuli to the lateral line system of fish in still water}

\subsection{Introduction}

The lateral line system of fish and some amphibians has been shown to be useful for extracting hydrodynamic information from its environment (Coombs and Montgomery, 1999). There is some evidence that the superficial lateral line system can be used for prey detection (Abdel-Latif et al., 1990), while other evidence suggests that it is the canal lateral line system which mediates detection and orienting responses (Coombs et al., 2001). To address this issue, the physics of the source signal and medium, the anatomy of the receptor organs, the neural encoding process, and the observed behaviors, must be analyzed in kind (for reviews see Dijkgraaf, 1963; Bleckmann, 1993; Coombs and Montgomery, 1999; van Netten, 2006).

The previous chapter developed the mechanical transduction process for superficial and canal lateral line system of the sculpin. The purpose of this chapter is to understand how the presence of the body and extended pectoral fins might affect the hydrodynamic information available to the lateral line. In particular, the ubiquitous 
dipole source (for example, Denton and Gray, 1982; Coombs et al., 1996; Coombs and Conley, 1997a, b; Kanter and Coombs, 2003; Curcic-Blake and van Netten, 2006; van Netten, 2005; Yang et al., 2006) will be the chosen hydrodynamic signal. Still water conditions will be used in this chapter, while the next chapter will consider dipole and body-wake signal distortions occurring in relative water motion.

Hassan (1993) was the first to examine the effect of the presence of the fish body on the pressure and velocity field of a vibrating sphere. The vibrating sphere was modeled as a potential source doublet, which creates a dipole field. He used slender body theory to represent axisymmetric fish shapes. The body presence is modeled using a combination of point sources, whose strength and location along the body axis are adjusted so as to have the velocity induced by the source dipole vanish at the surface. He showed that the body presence causes the pressure and velocity amplitudes to double at some locations along the fish body. In addition, the specific perturbations to the flow field are dependent on the exact location and orientation of the dipole source relative to the fish body.

In addition to any effects the presence of the body may have on altering the hydrodynamic flow, the anatomy and location of the receptor organs act to further this process. When there is relative water motion between the body and the surrounding fluid, a boundary layer develops where the motion of the fluid transitions from rest relative to the body to its full velocity in the surround flow. When the boundary layer thickness is a significant portion of the neuromast height, it is the dynamics within the boundary layer that governs the motion of the neuromast. In modeling the flow around superficial neuromasts, Kamijn (1988) has postulated that the neuromasts actually respond to the geometric mean of the velocity and acceleration of the fluid outside the boundary layer.

The canal neuromasts are further removed from the local hydrodynamic flow by residing in a subsurface canal. Water motion within the canal is forced to be aligned with the canal. Modeling the system as a damped harmonic oscillator being forced by the drag from the oscillating canal fluid, Kalmijn (1988) showed that canal neuromasts respond to the canal fluid velocity. This fluid velocity is proportional to 
the acceleration of the ambient water volume relative to the fish (Denton and Gray, 1983). Further work by van Netten (2005) has shown this model to be an inadequate explanation of the mechanics involved. It turns out that the physical structure of the canal and the effects of viscosity allow for a variety of flow profiles, which causes the canal to act as a first order filter for the accelerated fluid outside the boundary layer. Its low cutoff frequency of $20 \mathrm{~Hz}$ seems to contradict the fact that some fish have known responses to signals at frequencies as high as several hundred $\mathrm{Hz}$ (van Netten, 2005). However, the canal neuromasts also display resonance at certain frequencies, serving to boost the amplitude just as the physical nature of the canal diminishes it, allowing for an almost uniform response to a whole range of frequencies (van Netten, 2006).

Behavioral studies show that the lateral line canal system, and their encoded excitation patterns, plays an important role in dipole source localization (Coombs and Conley, 1997a, 1997b). Of attacks with the source at least $3 \mathrm{~cm}$ away, $75 \%$ of them occurred with the source axis off centered by at least 20 degrees in relation to the orientation of the fish body axis (Coombs and Conley, 1997b). Neurological studies have shown that the firing rate of nerves associated with individual canal neuromasts correspond to the modeled and measured pressure differences upon them (Coombs et al., 1996; Curcic-Blake and van Netten, 2006). Both the modeled and measured pressure differences in these studies were done subsequently to the neural measurements, and were made without the body present, though at the spatial locations where the body was previously placed.

This chapter develops a three-dimensional model of the sculpin, which includes a dorsal fin, pectoral fins, and a tail. The locations of the lateral line sensory organs on this model are based on the actual lateral line locations (see figure 3-2). Using CFD, the Navier-Stokes equations are solved to yield the full viscous, time-dependent solutions for the case of the vibrating sphere next to a sculpin body. Various configurations are considered, including duplicating (unpublished) results of an actual sculpin strike on an oscillating sphere. The series of calculations show explicitly how the fish body perturbs the lines of constant pressure of the dipole field, and how full 
use of the canal lateral line system (both sides of the fish) can work together to aid in prey localization. Another set of simulations that place the oscillating dipole in locations and orientations common to many studies is used to explicitly compare the effects of body parts on the received dipole signal to the lateral line system. The 3D results are compared with $2 \mathrm{D}$ results, in order to determine the effectiveness of $2 \mathrm{D}$ simulations (which provide considerable computational savings).

In addition, this chapter considers a method for modeling the boundary layer flow next to a wall created by a nearby dipole source. In some instances, where surface curvature is not extreme, the side of a fish may act similar to a localized portion of the wall. It utilizes Stokes' (1961) solution for a plate of infinite dimension oscillating in an unbounded fluid. In the case of an oscillating sphere, the dipole field creates a fluid of uneven velocity and pressure distributions, violating the assumptions of Stokes' solution. Nonetheless, this approximation is validated for points of maximum tangential velocity along the wall, using a variety of sphere sizes, locations, velocity amplitudes, and oscillation frequencies. From the approximation, a strain rate threshold for superficial neuromasts is defined which can easily be applied for cross study comparisons.

\subsection{Methods}

For the first case considered, a blinded sculpin rests on the bottom of a holding tank, with a chemically inert plastic sphere of $6 \mathrm{~mm}$ diameter close by. The sphere is attached to a thin steel shaft which extends into the water from a mini shaker (see Coombs and Conley (1997) for exact details). The sphere performs sinusoidal motions at $50 \mathrm{~Hz}$ along an axis that runs through the center of the mini shaker, for a series of bursts of $500 \mathrm{~ms}$ on and $500 \mathrm{~ms}$ off. The camera was placed under the tank, and circles of radius $3 \mathrm{~cm}, 6 \mathrm{~cm}$, and $9 \mathrm{~cm}$ were drawn around the centrally located sphere. The video stills chosen reflect the stopped locations in the sculpin's step-like approach to the target sphere.

To best see the effect that the fish body and fins have on the received dipole signal, 
three cases are compared: a body with fins extended, a body with fins retracted, and a virtual body with fins retracted. The virtual body is just the hydrodynamic information for a dipole source in an unbounded medium without any body present, calculated at locations identical to the real body cases.

\subsubsection{Computational domain: 2D strike case set up}

Numerical simulations were carried out using the commercial software packages, GAMBIT $^{T M}$, the mesh generator, and FLUENT ${ }^{T M}$ the solver (as explained in chapter 2). A $6 \mathrm{~mm}$ diameter cylinder was used in place of a sphere. For mesh construction, 20 nodes were equally spaced around the circumference, and the cylinder was centered in a deforming region, $3 \mathrm{~cm} \mathrm{x} 2 \mathrm{~cm}$ for the first 2 strike locations, and $1 \mathrm{~cm} \mathrm{x} 1 \mathrm{~cm}$ for the final location. Nodes were placed $2 \mathrm{~mm}$ along the border, and the inner region meshed with triangular cells. The cylinder velocity was set to $U_{0} \cos (\omega t)$ along the axis of vibration, with $U_{0}=1 \mathrm{~mm} / \mathrm{s}$, and the frequency of oscillation ( $\omega=2 \pi f$ ) set to $f=50 \mathrm{~Hz}$. By the no slip boundary condition, the fluid velocity at the cylinder surface is equal to the cylinder velocity. The amplitude of the cylinder motion is defined as $U_{0} / \omega$, which means the maximum displaced was $300+$ times less than 1 $\mathrm{mm}$. Therefore, the node displacement was minimal within the deforming zone.

The dimensions for the fish body were obtained from the cross-section of a Lake Michigan Mottled Sculpin. This gave a length of $7.6 \mathrm{~cm}$ and width of $2 \mathrm{~cm}$. A crosssection of the pectoral fins gave a thin slice of approximately $11 / 2 \mathrm{~cm}$ in length at roughly 45 degrees angle away from the body. Points around the body, $2 \mathrm{~mm}$ apart, were mapped to a grid. From these points, three sections of points were selected to represent idealized pore openings on a source side, back side, and front canal lateral line (see figure 4-1). The velocity normal to the body surface was forced to be zero. The body (both with fins and without) and cylinder were orientated to match experiment.

An outer rectangular boundary region, $375 \mathrm{~cm}$ x $100 \mathrm{~cm}$, was created to be large relative to the cylinder to minimize any boundary influences. Nodes were spaced 10 $\mathrm{cm}$ apart around the circumference. For boundary conditions, three sides had the 
velocity set to $0 \mathrm{~m} / \mathrm{s}$, and the fourth side had the pressure set to $0 \mathrm{~Pa}$. Triangular cells filled in the region containing the sculpin and the deforming zone (containing the cylinder).

The time step was 1/100th of an oscillation period, and 1000 time steps were used

to initialize the fluid field. This was to allow transients to die out, although only 15 time steps were all that was needed in the 2D simulations for the transients to decay. Finally, measurements were obtained at each of the nodes along the fish when the pressure field was at a maximum point in the oscillation cycle.

It is the pressure difference between pore openings to the canal lateral line that determines the fluid flow within the canal, and hence the effective stimulus being sought in this study. The pressure difference was calculated between pore openings, according to $\left(p_{2}-p_{1}\right) / 2 \mathrm{~mm}$. With the goal of highlighting differences between the source side and back side lateral lines in particular, it was thought important to have the order of differencing be in a symmetrical direction, in this case with the $p_{2}$ pore in any pair being closer to the tail. This was to reflect the possibility that the fish might be using an internal reference to compare the neural information it generates between the two sides of the fish. For the front lateral line, continuity in the plot was desired, and in the absence of knowing how the fish actually processes the information, a back side $\left(p_{2}\right.$ pore $)$ to source side ( $p_{1}$ pore) direction was chosen for the whole section (figure 4-1).

\subsubsection{Computational domain: 3D strike case set up}

A sphere $3 \mathrm{~mm}$ in diameter was created in $\mathrm{GAMBIT}^{T M}$. The sphere was fit with triangular faces, with each edge approximately $1 \mathrm{~mm}$ in length. This sphere was enclosed by a spherical deforming region of $15 \mathrm{~mm}$ radius, fit with triangular faces approximated $2 \mathrm{~mm}$ in length. The deforming volume was filled in with 13800 cells. The sphere velocity was set to $U_{0} \cos (\omega t)$ along the axis of vibration, with $U_{0}=7$ $\mathrm{mm} / \mathrm{s}$, and the frequency of oscillation $(\omega=2 \pi f)$ set to $f=50 \mathrm{~Hz}$. By the no slip boundary condition, the fluid velocity at the sphere surface is equal to the sphere velocity. The node displacement was minimal within the deforming volume. 


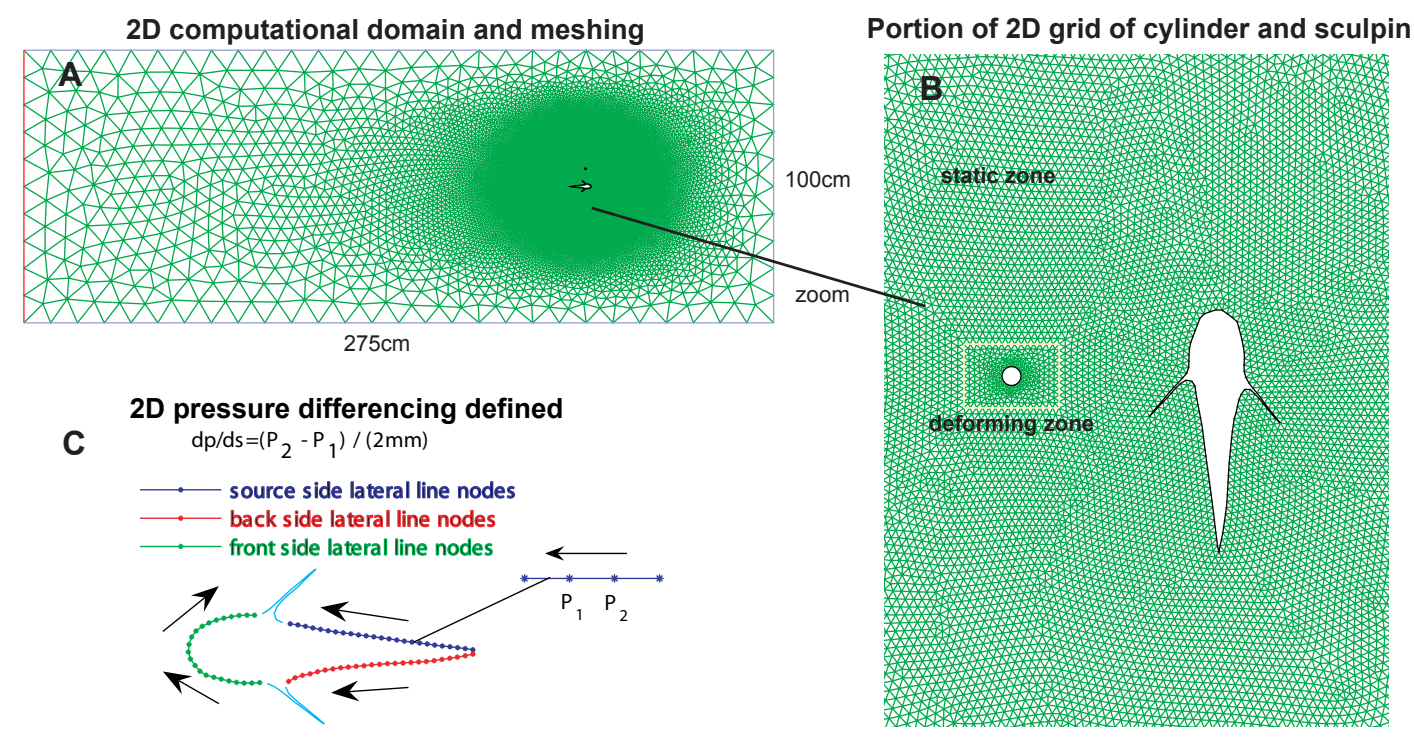

Figure 4-1: (A) The computational domain for the 2D cylinder and sculpin. (B) A zoomed in view shows the region of mesh around the cylinder which is allowed to deform as the cylinder oscillates, whereas the remainder of the region is static. (C) Node locations around the sculpin body, where the pressure is measured. The conventions for calculating the pressure difference are identified by the arrow direction. The pressure at the arrow tip pore opening is subtracted from the pressure at the arrow tail pore opening. The distance between all pore openings is approximately 2 $\mathrm{mm}$. 
A sculpin was constructed using $\mathrm{GAMBIT}^{T M}$, to approximately match the dimensions of the real fish. The length of the main body of the fish is $8 \mathrm{~cm}$, with a portion of the tail extending another $1 \mathrm{~cm}$. The body is $2 \mathrm{~cm}$ in diameter at its widest point, just in front of the pectoral fins. The dorsal fin extends about a $1 / 2 \mathrm{~cm}$ above the fish in its front, and about $1 \mathrm{~cm}$ above in its rear. The pectoral fins and tail fin are $0.5 \mathrm{~mm}$ thick, while the dorsal fin is $1 \mathrm{~mm}$ thick. The fish was meshed with triangular faces, each edge $2 \mathrm{~mm}$ in length (except for fin edges, which kept their thinner dimensions). The sculpin was placed $1 \mathrm{~mm}$ above the floor. This was so that the sculpin body had its own mesh, independent of the box boundaries (see figure 4-2).

On the wall beneath the sculpin, a rectangle of $15 \mathrm{~cm} \mathrm{x} 10 \mathrm{~cm}$ was selected for meshing to match the spacing found on the body of the fish. Nodes were spaced 2 $\mathrm{mm}$ apart, and triangular cells filled this region. This was done to keep most the cells around the fish and sphere approximately equal in size. This region was oriented with the sculpin, and became part of the overall floor boundary.

A box of dimension $60 \mathrm{~cm} \mathrm{x} 40 \mathrm{~cm}$ x $10 \mathrm{~cm}$ was formed to represent the experimental tank boundaries. The edges were given nodes spaced $1 \mathrm{~cm}$ apart. The side that was the floor was filled in with triangular cells (in order to mesh smoothing with the region under the sculpin), while the other five sides were filled in with squares 1 $\mathrm{cm} \times 1 \mathrm{~cm}$. The inside of the box was filled in with quadrilateral cells.

The sphere and wall where given solid boundary conditions, one side of the box was made to have zero pressure, and the other four sides given conditions of symmetry. These boundary conditions were selected to maximize the presence of the wall, while minimizing the effects of all other sides. This was also accomplished by choosing such large dimensions for the box, relative to the sphere size. The sphere was placed 1.6 $\mathrm{cm}$ above the floor, slightly above the height of the actual lateral line. The body (both with fins and without) and sphere were orientated to match experiment.

FLUENT $^{T M}$ was used to solve the Navier-Stokes equations. The time step was set to $1 / 100$ th of the oscillation period, and 1000 time steps were used to initialize the fluid field. Measurements were obtained at each of the nodes over the entire fish body. 
For the 3D case, the pressure gradient can be calculated over the entire body. However, the lateral line on fish is not spread over the whole body, but follows a distinct pattern (figure 4-2). Therefore, two cases were considered: the actual lateral location, as seen on the mottled sculpin (see figure 3-2), and an imaginary lateral line located at the midplane of the fish, for more direct comparison with the $2 \mathrm{D}$ case. In both cases, the pressure difference was calculated according to $\left(p_{2}-p_{1}\right) / 2 \mathrm{~mm}$. For the midplane case, the direction of differencing was identically matched to the $2 \mathrm{D}$ case. For the actual lateral line location, the goal of seeing how the pressure gradient changes continuously from one side of the fish to the other, the order of differing was from the back side tail, around the front, and towards the source side tail.

To obtain the pressure at points along the midline, nodes within $0.8 \mathrm{~mm}$ on either side of the line were selected. These nodes were not evenly spaced around the midline, and sometimes two nodes on opposite sides of the midline would be very close to each other, skewing any pressure difference calculations. Therefore, a spline curve was fit to the pressure values along each section of the lateral line (back, front, and source sides), sampled every $3 \mathrm{~mm}$, and low pass filtered (by averaging adjacent values). Finally, pressure points were sampled every $2 \mathrm{~mm}$, and then used in the previously described pressure differencing formulas.

To obtain the pressure at points along the actual lateral line, node locations were selected by hand. These locations approximately followed what might be considered the true lateral line, and all strike cases used 3 nearby pressure values to interpolate to these selected locations. Since the locations were not evenly spaced, a spline curve for the pressure values was fit to each of the lateral line sections (back side, back infraorbital, source infraorbital, and source side), sampled every $1 \mathrm{~mm}$, and low pass filtered (by averaging 2 adjacent values). Finally, pressure points were sampled every $2 \mathrm{~mm}$, and then used in the previously described pressure differencing formulas. 


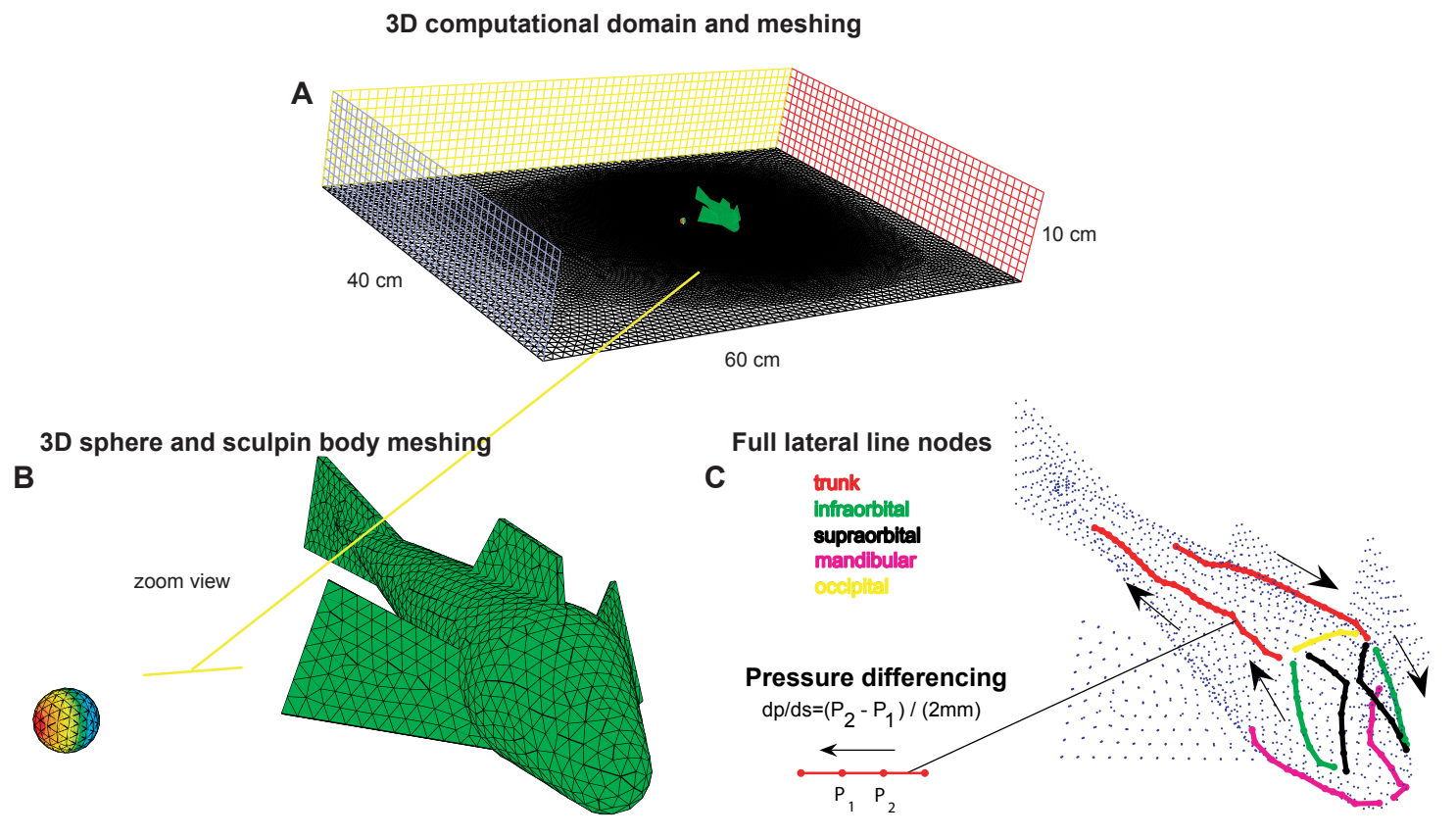

Figure 4-2: (A) The computational domain for the sphere and sculpin. (B) A zoomed in view shows the meshing on the surface of the sphere and sculpin, with edge lengths approximately $1 \mathrm{~mm}$ apart on the sphere and $2 \mathrm{~mm}$ apart on the sculpin. The area immediately around the sphere is allowed to deform (not shown), while the rest of the mesh remains static. (C) Node locations around the sculpin body, where the pressure is measured, including those points which fall directly on the sculpin's lateral line. The conventions for calculating the pressure gradient are identified by the arrow direction. The pressure at the arrow tip pore opening is subtracted from the pressure at the arrow tail pore opening. The distance between all pore openings is approximately $2 \mathrm{~mm}$. Full lateral line designation: Trunk (red), Occipital (yellow), Infra-orbital (green), Supra-orbital (black), Mandibular (magenta). 


\subsubsection{Computational domain: 3D canonical dipole localiza- tion case set up}

A few cases were selected to match what is seen in the literature (e.g. Denton and Gray, 1982; Coombs and Conley, 1996; Coombs et al., 2001; Curcic-Blake and van Netten, 2006) - that of an oscillating sphere with its axis parallel and perpendicular to the long axis of the fish. These cases are instructive in recognizing the standard pressure gradient patterns seen near a wall, and also for comparing canal and superficial lateral line signals.

The sphere was placed at approximately 3 diameters equidistant above the side lateral lines, located posterior to the pectoral fins. The sphere was also placed 4 and 7.5 diameters lateral to the fish, at one location in front of the pectoral fin (and next to the infraorbital/front canal lateral lines), and at another location behind the pectoral fin (and next to the trunk canal lateral lines). The sphere diameter is less than 1/10 BL (body lengths), and the deforming region radius was shortened to 4 radii to allow for closer placement of the sphere to the body.

FLUENT $^{T M}$ was used to solve the matrix of equations. The time step was $1 / 100$ th of an oscillation period, and 200 time steps were used to initialize the fluid field. Measurements were obtained at each of the nodes over the entire fish surface, and the pressure difference was calculated for the actual lateral line location. Unlike the strike cases, no pressure interpolation or smoothing was performed because node locations matched the hand selected locations.

\subsubsection{Analytical models for the velocity profile and strain rate at a wall due to a dipole source}

Potential flow theory is valid in an incompressible, inviscid, and irrotational medium. For a sphere of radius $a$, oscillating at angular frequency $\omega=2 \pi f$, there is a small region next to the sphere surface where viscous forces dominate or balance inertial

forces. The height of this boundary layer is on the order $\delta \sqrt{2 \nu / \omega}$ (Schlichting, 1979), where $\nu$ is the kinematic viscosity of the fluid. For example, a sphere oscillating at 
$f=50 \mathrm{~Hz}$ has strong viscous effects for a distance of at least $\delta 80 \mu \mathrm{m}$, though the effects do extend further. In this region and beyond, the fluid is rotational, being mitigated in amplitude in proportion to $\exp (-(r-a) / \delta)$, where $r$ is the radial distance from the center of the sphere (van Netten, 2006). Already after $6 \delta$ with the example given (which would be $0.5 \mathrm{~mm}$ past the sphere surface), the rotational effects are diminished over 400 times. Beyond this region, the medium is effectively inviscid and irrotational. However, the transition from the viscid, rotational region to the inviscid, irrotational region results in amplitude and phase changes relative to the oscillating sphere. These will appear as correction terms to what would otherwise be an analytical solution for an oscillating sphere in a completely inviscid, irrotational fluid medium.

In the outer inviscid, irrotational region of interest, the velocity potential equation for an oscillating sphere in an unbounded medium is (van Netten, 2007; eg. Stokes, 1851; Lamb, 1931)

$$
\phi_{\mathrm{sph}}=-C \frac{a^{3}}{2 r^{2}} U_{0} \cos \theta \cos (\omega t-\zeta)
$$

The $\mathrm{C}$ and $\zeta$ terms are amplitude and phase correction terms, respectively, given as (van Netten, 2007)

$$
C=\sqrt{C_{1}^{2}+C_{2}^{2}} ; \zeta=\arctan \left(C_{2} / C_{1}\right)
$$

where

$$
C_{1}=1+\left(\frac{3 \delta}{2 a}\right), C_{2}=\left(\frac{3 \delta}{2 a}\right)\left[1+\left(\frac{\delta}{a}\right)\right]
$$

The amplitude correction term (C) approaches unity when the boundary layer thickness $(\delta)$ is small compared to the radius (a). This more readily occurs with increase in frequency, since $\delta 1 / \sqrt{\omega}$. Likewise, the phase correction term $(\zeta)$ approaches zero with increase in frequency and radius. For typical experimental values, such as a sphere of radius $3 \mathrm{~mm}$ oscillating at $50 \mathrm{~Hz}$, the correction terms are small $(\mathrm{C} 1.04$ and $\zeta 0.04$ radians).

$\phi$ is called the velocity potential because the velocity components are found by taking the gradient, $\vec{v}=\nabla \phi$. The pressure is given by the Bernoulli equation for potential flow, which reduces to $p \approx-\rho \partial \phi / \partial t$ when the gradient squared term is 
small in comparison to the time changing terms (and with gravity ignored).

These equations for pressure and velocity are valid in an unbounded medium, but they will certainly change with the presence of a fish body. For an oscillating sphere next to the side of a fish, the fish's surface might appear to be a wall to the immediate hydrodynamic field. Clearly, there will be a weakness in this approximation at regions of strong curvature or blocked flow (such as at fin insertion points). However, over much of the side surface of the fish, this approximation will likely be valid, and this is a simplification worth exploring.

When the flow is potential in nature, the method of images may be employed to represent an oscillating dipole sphere near a wall by superposing a second, mirrored oscillating dipole sphere an equidistant across an imaginary plane where the wall would reside. Essentially, this results in doubling the magnitude for $\left.\phi\right|_{\text {wall }}$, and by extension $\vec{v}, p$, and $\nabla p$ (see chapter 2 ).

The strain rate depends on the velocity gradient, and it is at the body surface that the gradient will be most significant. In Cartesian coordinates, the velocity vector tangent to any point on the surface will have $\mathrm{x}, \mathrm{y}$, and $\mathrm{z}$ components. The definition of strain rate used by FLUENT ${ }^{T M}$ is given as

$$
\begin{aligned}
S^{2}=2\left[\left(\frac{\partial u}{\partial x}\right)^{2}+\left(\frac{\partial v}{\partial y}\right)^{2}+\left(\frac{\partial w}{\partial z}\right)^{2}+\right. & \left.\frac{1}{2}\left(\frac{\partial u}{\partial y}+\frac{\partial v}{\partial x}\right)^{2}+\frac{1}{2}\left(\frac{\partial u}{\partial z}+\frac{\partial w}{\partial x}\right)^{2}+\frac{1}{2}\left(\frac{\partial v}{\partial z}+\frac{\partial w}{\partial y}\right)^{2}\right] .
\end{aligned}
$$

To determine the strain rate, the local boundary layer velocity profile is needed. This is very difficult to measure experimentally, and difficult to obtain computationally. However, it may be possible to obtain an approximate boundary layer velocity profile by using Stokes (1851) solution for a uniform fluid oscillating over a stationary wall. The idea is that the wall (fish body) will help align the flow from the dipole field tangentially. By using the approximation of a locally uniform fluid flow, the maximum component of the strain rate will be in the direction of this flow, dominating other 
components. Then an approximate strain rate threshold can be defined as

$$
S_{\text {approx }} \approx \frac{\nabla U}{\nabla L}=\frac{\text { tip velocity }- \text { base velocity }}{\text { length of cupula }} .
$$

It is important to note that the tip velocity assumes that the flow is in the direction of hair cell polarization, and that the base velocity is zero by the no-slip condition. Stokes solution is actually for an oscillating plate in stationary fluid, but by changing the frame of reference to the plate, the desired result is achieved. The velocity profile is given as

$$
u=U_{\infty}\left[e^{-y \sqrt{\frac{\omega}{2 \nu}}} \cos \left(\omega t-y \sqrt{\frac{\omega}{2 \nu}}\right)-\cos (\omega t)\right],
$$

where $U_{\infty}$ is the fluid velocity just outside the boundary layer, and y is the distance from the wall. The strain rate at the wall may be approximated as the magnitude of the principal strain rate component,

$$
\left.S_{\max }\right|_{\text {wall }} \approx|d u / d y|_{\text {wall }}=(\sqrt{2}) U_{\infty} \sqrt{\frac{\omega}{2 \nu}},
$$

where the $\sqrt{2}$ factor is the maximum value found at $t=\frac{3 \pi}{4} \mathrm{~s}$, from the time dependent terms. Allowing the boundary edge velocity $\left(U_{\infty}\right)$ to equal the magnitude of the tangential velocity at the wall as determined from potential flow theory ( $2\left\|\left.\vec{v}\right|_{\text {wall }}\right\|$ ), the velocity profile becomes

$$
u=U_{0}\left(\frac{a}{r}\right)^{3}\left[e^{-y \sqrt{\frac{\omega}{2 \nu}}} \cos \left(\omega t-y \sqrt{\frac{\omega}{2 \nu}}\right)-\cos (\omega t)\right] * C(a, \omega) * f(\alpha),
$$

where $f(\alpha)$ is an amplitude term that results when the sphere oscillates in a direction that makes an angle of $\alpha$ with the wall-axis. $f(\alpha)$ varies between 1 ( $\alpha=0$ radians) and 0.86 ( $\alpha=\pi / 2$ radians), and is determined by taking the maximum value of the normalized potential flow solution for the flow next to wall, induced by a sphere oscillating at an angle of $\alpha$ to the wall. Finally, an analytic expression for the strain 
rate at the wall is given as

$$
\frac{\left.S_{\max }\right|_{\mathrm{wall}}}{U_{0} \sqrt{\omega / \nu} * C(a, \omega)} \approx \frac{a^{3}}{r^{3}} * f(\alpha) .
$$

\subsubsection{Numerical determination of the strain rate at a wall due to an oscillating sphere}

Using the mesh generator program, GAMBIT ${ }^{T M}$, and the solver, FLUENT $^{T M}$, a series of trials were carried out to verify the approximate velocity profile and strain rate at the wall. Each trial used a different set of values for the parameters (as in the literature), under the assumption that regardless of the values chosen, all results would fall onto the curves predicted by the analytical models. The parameters had ranges of $7 \mathrm{~mm} / \mathrm{s} \leq U_{0} \leq 314 \mathrm{~mm} / \mathrm{s}, 2.5 \mathrm{~mm} \leq \mathrm{a} \leq 18 \mathrm{~mm}, 25 \mathrm{~Hz} \leq \mathrm{f} \leq 75 \mathrm{~Hz}$, and $1.1 \mathrm{~cm} \leq \mathrm{r} \leq 20 \mathrm{~cm}$

A grid was created for each trial separately, with spatial resolution increased until grid convergence was achieved. At convergence, box dimensions ranged from 16.67x16.67x8.33 sphere diameters for the sphere located 1.14 diameters from the wall, to $120 \times 120 \times 180$ sphere diameters for the sphere located 40 diameters from the wall. At the floor of each grid, a boundary layer was created with fine vertical resolution (i.e. gridpoints at $0,5,13,24,39,61, \ldots$ microns), and with horizontal resolution most resolved in the center of the floor (i.e. as low as $0.7 \mathrm{~mm}$ at the center vs. at most $15.4 \mathrm{~mm}$ near the edges). The floor was meshed with quadrilateral cells. The sides of the box had resolution that matched that of the floor at one end, with increasing intervals up to $1 \mathrm{~cm}$ spacings at the top. The sides, top, and interior of the box was meshed with tetrahedral cells.

The sphere surface is meshed with triangular cells, with sides of approximate length 0.7-1 mm. A deforming region adjacent to the sphere was filled with tetrahedral cells, whose side lengths grow from $1 \mathrm{~mm}$ at the sphere surface to $2 \mathrm{~mm}$ at the edge of the region located up to a distance of 2-8 radii away. The cell centroids adjoining the sphere surface are located at heights of $0.2-0.5 \mathrm{~mm}$ above the surface. This is outside 
of the boundary layer that forms over the surface as the sphere oscillates, and therefore does not correctly resolve the flow beneath. Essentially, this is equivalent to setting the amplitude correction term to $\mathrm{C}=1$ and the phase correction term to $\zeta=0$. For the numerical simulations, it was thought worthwhile to allow for this approximation in order to save on computation complexity. However, in actual experiments, the correction factors should be considered.

The time step was at least 1/100th of an oscillation period, and 200 time steps were used to initialize the fluid field. This was to allow transients to die out, although only a few time steps were all that was needed. Measurements were obtained at different points within the oscillation period for different trials, to allow for more general validation. The maximum strain rate along the wall was measured, regardless of where that point actually occurred. According to potential flow theory, when the sphere is oscillating parallel to the wall, the point of maximum tangential velocity is centrally located at the point where a line normal to the wall intersects the center of the sphere. When the axis of sphere oscillation makes and angle of $\alpha$ with the wall, this point of maximum tangential velocity migrates away from the central point.

\subsubsection{Numerical determination of the pressure gradient at a wall due to an oscillating sphere}

At the wall (fish body) itself, a boundary layer will be generated by the oscillating fluid, and that will greatly affect the velocity near the surface, and therefore the strain rate. However, when the boundary layer is small relative to the body (wall) thickness, the pressure is uniform throughout the boundary layer. If the pressure were all that was desired, the boundary layer would not need to be modeled, which would be a large savings in computation. The velocity calculated outside the boundary layer would be essentially that determined from a purely potential calculation (no viscosity). This is satisfactory for showing the general pattern of flow over the fish body, and for

determining what effect body parts have on the flow. Once the simulation of a sphere oscillating next to a wall with grid resolution matching that for the fish body is 
validated against potential flow theory, it will be assumed to produce the correct flow field when the fish body is added.

A sphere $3 \mathrm{~mm}$ in diameter was created in $\mathrm{GAMBIT}^{T M}$, at distances $11 \mathrm{~mm}, 21$ $\mathrm{mm}$, and $51 \mathrm{~mm}$ from a wall. The sphere was fit with 260, 260, and 266 triangular faces, with each edge approximately $1 \mathrm{~mm}$ in length. This sphere was enclosed by a spherical deforming region of $9 \mathrm{~mm}, 18 \mathrm{~mm}$, and $18 \mathrm{~mm}$ radius, filled with 3863 , 22229, and 20761 cell volumes. Its surface contained 598, 2338, and 2336 triangular faces, with each edge approximate $2 \mathrm{~mm}$ in length.

On the wall beneath the oscillating sphere, a square with sides $20 \mathrm{~cm}$ in length and nodes space $2 \mathrm{~mm}$ apart was filled in with 22490 triangular faces, each with edge approximately $2 \mathrm{~mm}$ in length. This square was centered on a wall with overall dimensions of $60 \mathrm{~cm} \mathrm{X} 60 \mathrm{~cm}$, with nodes spaced $1 \mathrm{~cm}$ apart, and then filled in with additional 48098 triangular faces. The projection of the sphere center onto the wall plane shows it to be $5 \mathrm{~cm}$ off center (to allow for center placement of a sculpin body in the next experiment).

Five other sides with dimensions $60 \mathrm{~cm} \mathrm{X} 60 \mathrm{~cm}$ were added to form a box (representing an experimental tank), with nodes spaced $1 \mathrm{~cm}$ apart, and filled in with 3600 square faces each. The entire rest of the volume (outside the deforming region and within the box walls) was filled in with 1261158, 1270133, and 1273711 cell volumes.

The sphere and wall where given solid boundary conditions, one side of the box was made to have zero pressure, and the other four sides given conditions of symmetry. These boundary conditions were selected to maximize the presence of the wall, while minimizing the effects of all other sides. This was also accomplished by choosing such large dimensions for the box, relative to the sphere size.

The time step was set to $1 / 100$ th of the oscillation period. The sphere motion was set to $U_{0} \cos (\omega t)$, with $U_{0}=7 \mathrm{~mm} \mathrm{~s}^{-1}$, the radius of the sphere set to $a=3 \mathrm{~mm}$, and the frequency of oscillation $f=50 \mathrm{~Hz}$.

In running numerical simulations, all units are given their actual dimensional values. Therefore, the dimensions of the fish, sphere, cylinder, and boundaries are meant to reflect what is found in the general literature. However, for broader generalization, 
the results are presented in their non-dimensional form. Dividing each equation by its normalizing constant leaves a normalized geometric expression, which is specific to each case, body shape, and orientation. The surface strain rate equation was already given. The equation for pressure gradient at the wall is

$$
\frac{\nabla p_{\text {wall }}}{\rho \omega U_{0} * C(a, \omega)}=\frac{a^{3}}{r^{3}} * f(\alpha),
$$

where $f(\alpha)$ was previously given.

\subsection{Results}

\subsubsection{Numerical validation for the pressure and pressure gra- dient signals at a wall due to an oscillating sphere}

The numerical results for the pressure and pressure gradient fields show excellent agreement with the predicted calculations of a sphere next to a wall using potential flow theory (figure 4-3). For the pressure gradient calculations, the pressure curves were re-sampled for even spacing using a spline function, and low pass filtered, before being differenced. This was done to remove numerical noise, and had the result of reducing the amplitude of the pressure gradient only slightly.

A dipole source will produce a signature spatial pressure gradient (figure 4-3C) along the lateral line, providing information about the location of the source relative to the body. For a spherical dipole source oscillating parallel to the lateral line, the perpendicular bisection of its location with the long axis of the fish matches the location of the pressure gradient peak. Its distance away from the body axis is equal to $(1 / \sqrt{2})$ times the distance between the zero crossings (Curcic-Blake and van Netten, 2006). In a similar manner, when the spherical dipole oscillates perpendicular to the lateral line, the pressure gradient zero-crossing marks the perpendicular bisection of the sphere location and the long axis of the fish. The distance between the pressure gradient peaks is equal to the source distance (Curcic-Blake and van Netten, 2006).

In this manner, direct information is encoded about the location of the dipole source 
when it is on a plane level with that of the lateral line.

\subsubsection{Numerical validation for the boundary layer velocity profile and strain rate at a wall due to an oscillating sphere}

Figure 4-4 presents a sampling of trials whose parameters differ significantly from each other. A range of distances, oscillation frequencies, and velocity amplitudes are shown so that authors of experimental studies may have some confidence that the results presented might be applied to their own work. For the cases presented, the sphere axis of oscillation is parallel to the wall.

The velocity magnitude within the boundary layer has been normalized by $U_{\infty}$, the velocity calculated from potential flow theory, and assumed to be the actual velocity just out of range of viscous effects. Data points of the $u$ velocity, as determined from numerical simulation, are plotted against the predicted velocity profile (equation 4.8). The prediction is that the component of the flow aligned with the projected axis of oscillation should dominate over the velocity components. The plots show excellent agreement between the predicted and numerically determined velocities within the boundary layer.

The strain rate data points, as calculated from numerical simulation and through the use of the complete strain rate formula (equation 4.4), are plotted against the

analytical approximation of the strain rate $(S \approx|d u / d y|)$. Remember that the strain rate is by definition a positive value, representing deformation of the fluid particles. The data shows excellent agreement with the theory, though it is not absolutely certain that the boundary layer grid was fine enough to resolve all cross components of the actual strain rate (equation 4.4).

To verify that the boundary layer velocity profile is valid under changes in direction of oscillation of the sphere, figure 4-5 presents the wall strain rate as determined from all numerical trials plotted against the analytically determined wall strain rate (equation 4.9). If the velocity profile is different from that predicted, the numerically 

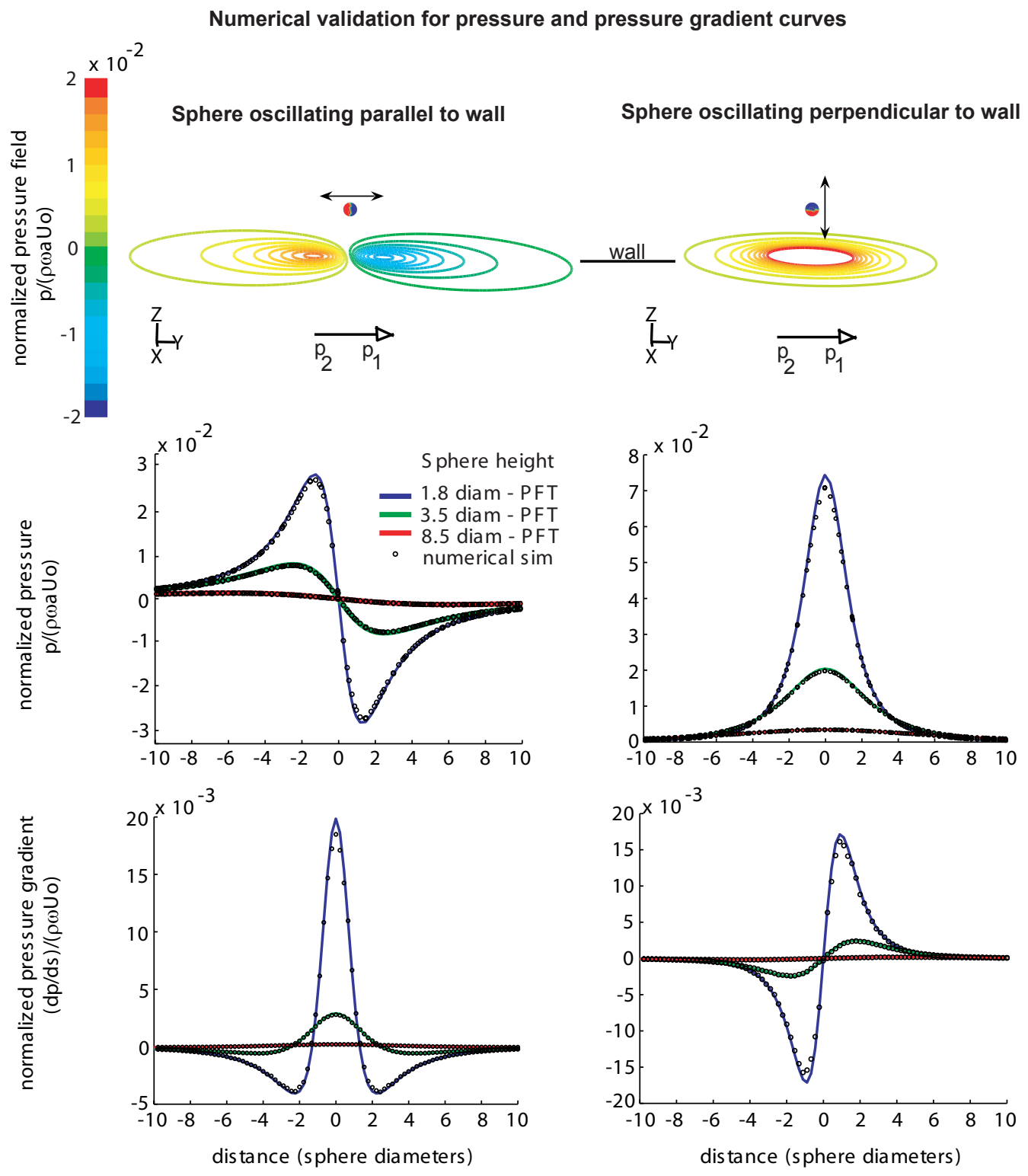

Figure 4-3: Instantaneous snapshots of the pressure field and calculated pressure gradient for a sphere oscillating (A) parallel, and (B) perpendicular to a wall. The sphere is located at heights of $1.8,3.5$, and 8.5 diameters from the wall. Numerical results are in agreement with potential flow theory, though slight differences become more apparent the closer the sphere is to the wall. The pressure gradient patterns shown are canonical for dipole source localization, with one shaped like a 'Mexican hat' (Coombs and Connelly, 1997b), and the other like a "heartbeat." 
determined wall strain rate cannot possible match either. However, the normalized results again show excellent agreement.

These results should allow for the theory to be applied under a number of different situations, for cross study comparisons. In addition to any experimentally determined velocity thresholds, the approximate strain rate threshold may be used when the height of the superficial neuromasts is also known, or the wall strain rate threshold may be used when there is only source information. This theoretical treatment of the velocity boundary layer might expect to be valid on surfaces that most approximate a wall, namely the boundary layer height is small relative to the curvature of the surface. Where there is strong curvature, the flow will most certainly be accelerated, and the assumptions for this theory are violated. But the results can be generalized beyond those due an oscillating sphere, since any oscillations in a fluid next to a flat surface will likely have similarities with Stokes flow within the boundary layer.

\subsubsection{Sculpin strike cases}

Figures 4-6 and 4-7 present the results of the presence of the fish body (pectoral fins extended and retracted) and absence of a fish body (virtual body) on the hydrodynamic field of an oscillating cylinder and sphere respectively. In the fish's starting location (strike position 1, figure 4-6A, 4-7A), the sphere is just under a body length away, with the relative angle between the axis of oscillation and the longitudinal body axis of about 30 degrees. In the second strike position (figure 4-6B, 4-7B), the sphere is also under a body length away, but not located laterally to the pectoral fin insertion point and oscillating in a direction perpendicular to the long axis of the fish body. In the third strike position (figure 4-6C, 4-7C), the source is closer to front of the fish

body, with the relative angle between the axis of oscillation and the long axis of the body about 150 degrees.

With the body present, the lines of iso-pressure terminate on the body, and are concentrated around curved regions and pointed parts of the body. These local concentrations of pressure cannot be predicted without the body present, and so the results using potential flow theory without the body present do not show them. The 


\section{Numerical validation of the boundary layer velocity profile at the wall due to an oscillating sphere}

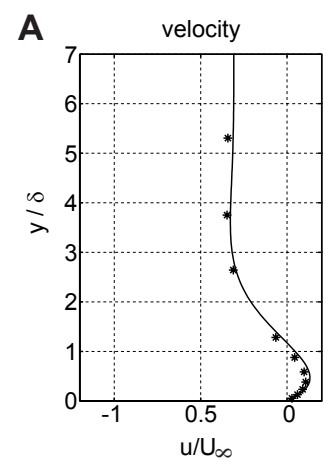

$\mathrm{f}=30 \mathrm{hz}$ dist=10 radii $\mathrm{U}_{0}=0.04 \mathrm{~m} / \mathrm{s} \quad U_{\infty}=40 \mu \mathrm{m} / \mathrm{s} \quad 0.04 \mathrm{sec}$

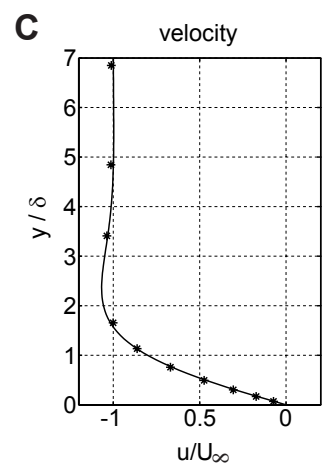

$\mathrm{f}=50 \mathrm{hz}$ dist $=3.67 \mathrm{radii} U_{0}=0.007 \mathrm{~m} / \mathrm{s} U_{\infty}=141.9 \mu \mathrm{m} / \mathrm{s} \quad 0.04 \mathrm{sec}$

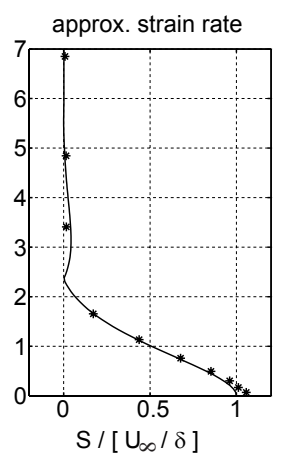

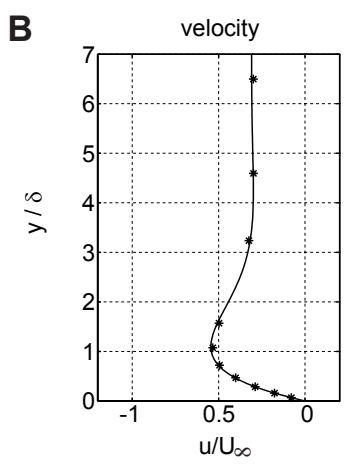

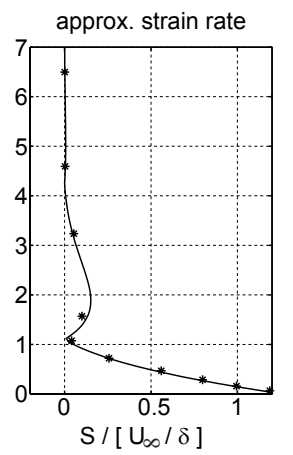

$\mathrm{f}=45 \mathrm{hz}$ dist=20 radii $U_{0}=0.1 \mathrm{~m} / \mathrm{s} \quad U_{\infty}=12.5 \mu \mathrm{m} / \mathrm{s} \quad 0.04 \mathrm{sec}$

D
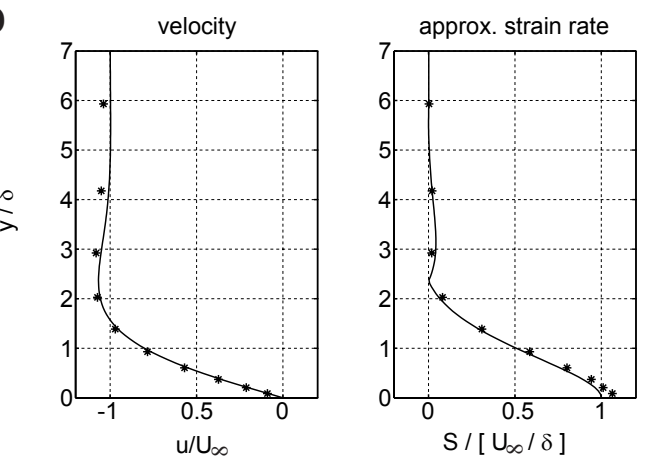

$\mathrm{f}=75 \mathrm{hz}$ dist $=40$ radii $U_{0}=0.03 \mathrm{~m} / \mathrm{s} \quad U_{\infty}=0.5 \mu \mathrm{m} / \mathrm{s} \quad 0.04 \mathrm{sec}$

Figure 4-4: The boundary layer velocity profile at the wall, and calculated strain rate, due to a parallel oscillating sphere, is validated through numerical simulation. A variety of distances, source amplitudes, frequencies, and sphere diameters are used to highlight the range of its application. The profiles are presented at different times within their natural periods: (A) $2 \pi / 5$, (B) $8 \pi / 5$, (C) $2 \pi$, and (D) $2 \pi$. 


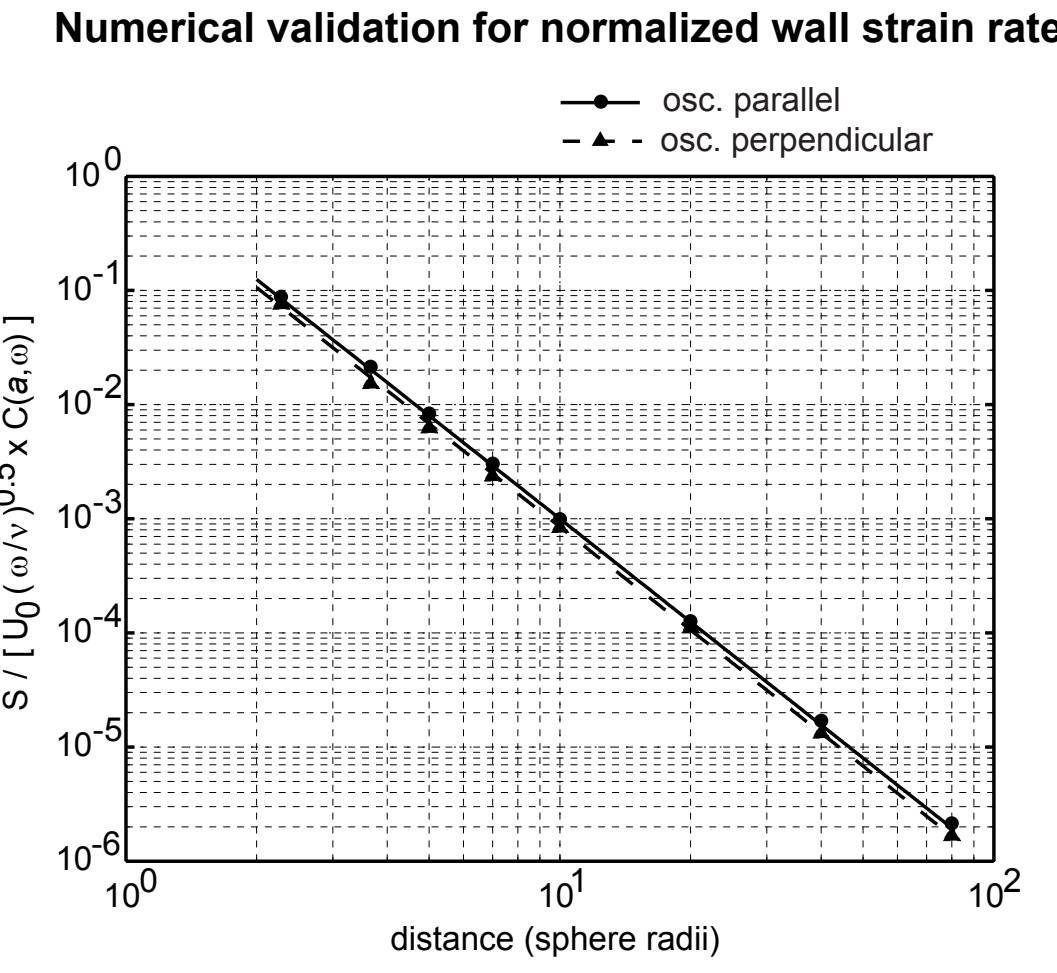

Figure 4-5: Numerical validation for determining the wall strain rate due to a nearby oscillating sphere, with $U_{\infty}$ calculated from potential flow theory as the wall slip velocity. The solid and broken black lines are analytical results due to a sphere oscillating parallel and perpendicular to the wall, respectively. The dots and triangles are numerical simulation results using the full strain rate formula (equation 4.4) at the wall surface, at the points of maximum tangential velocity. 
presence of the body also slightly shields a region of water from the oscillating source. This creates a region of more constant pressure along the side opposite the source, which results in a pressure difference closer to zero. The iso-pressure contour lines of the hydrodynamic field without a body present are of course undisturbed, and cannot predict where the shielded regions will occur.

When fins are extended, there is a small, localized pocket between the fins and the side of the body where the magnitude of the flow field is reduced. The effect is to calm the flow, and this causes the pressure differences seen by the midplane lateral line on both the source side and back side to be reduced near the fin insertion point. This is in contrast to the case with a streamlined body present, where only the body curvature itself determines how the pressure field is affected. This effect is expected to be more significant for the fish in a moving flow. The overall effect of this shadow zone is weaker (but still noticeable) in the 3D midplane case, because the flow field extends over and under the fins, as well as around them.

Magnitude differences around the body are directly related to the location of the dipole source. These differences show up strikingly between the side of the fish directly exposed to the source, and the shielded side. However, equally noticeable are the differences in magnitude seen between the head and tail of the fish. In the starting strike position, the magnitude for the pressure gradient is larger for the tail than for the front of the fish, and stronger for side closest to the source, indicating the sources location behind and to the side of the fish. In the second strike position, the order of magnitude for the pressure gradient is similar for the front and tail, though either side of the fish still shows a clear difference, indicating that the source is lateral to the fish. In the third strike position, the head of the body has moved much closer to the source, while the tail tip is yet further away. The pressure difference more than quadruples for the front lateral line, while there is only a slight decrease near the tail tip. This is unambiguous information for the fish that it is heading in the direction of the source.

The fin extension and retraction results are different for the $2 \mathrm{D}$ and $3 \mathrm{D}$ cases. Whereas there are clear differences between results for fins extended and retracted 
in the 2D case (figure 4-6) based on the relative orientation of the fish and dipole, there is not this detectable difference for the 3D cases (figure 4-7). This difference occurs because the flow is less perturbed in the 3D cases were the body occupies as smaller percentage of the overall flow field domain. However, when a background flow is introduced, there will likely be significant differences.

Figure 4-8 shows the results of having the actual lateral line be located above the midplane, and around (rather than through) the pectoral fins. In this third strike position, the back and source side trunk lateral lines, along with the mandibular lateral lines, capture much of the same information that an imaginary midplane lateral line would. The orientation of the back side supraorbital lateral line locations distorts the received signal, providing new information that is likely valuable to the fish for dipole source localization.

\subsubsection{Canonical cases: the signal to the canal lateral line system}

The results for a sphere oscillating parallel and perpendicular to the side of a sculpin are presented in figure 4-9. It is of one instant in time, when the pressure is at its maximum around the body. The coordinate system has its origin located at the nose of the sculpin, located 0.12 BL (body lengths) above the floor. When looking down on the fish, the nose points North, the tail points South, the right lateral side points East, and the left lateral side points West. The $\mathrm{x}$-axis is aligned with the rostrocaudal axis of the fish, with positive in the direction of the tail. The y-axis is aligned with the lateral axis of the fish, with positive toward the right side of the fish. The z-axis is aligned with the dorsoventral axis of the fish, with positive toward the dorsal side of the fish. The floor plane is parallel to the xy-plane.

In cases $\mathrm{A}$ and $\mathrm{D}$, the sphere is located at $(0.56,0,0.33) \mathrm{BL}$. For cases $\mathrm{B}$ and $\mathrm{E}$, the sphere is located at $(0.56,0.33,0.11) \mathrm{BL}$. In cases $\mathrm{C}$ and $\mathrm{F}$, the sphere is located at $(0.22,0.56,0.11) \mathrm{BL}$. In cases $\mathrm{A}, \mathrm{B}$, and $\mathrm{C}$ the sphere is oscillating parallel to the $\mathrm{x}$-axis, and in cases D, E, and F, the sphere is oscillating parallel to the $\mathrm{y}$-axis. 
Sculpin experiment

A Position \#1

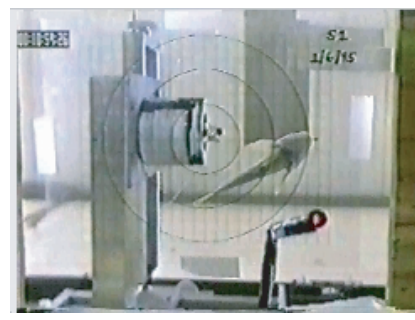

bottom view - looking up through the clear floor

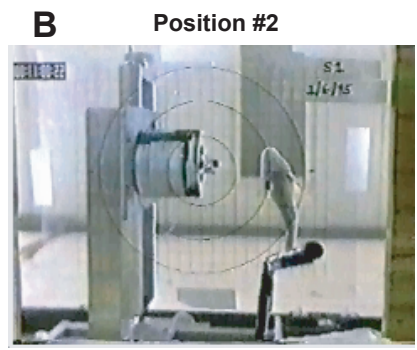

2D numerical simulations
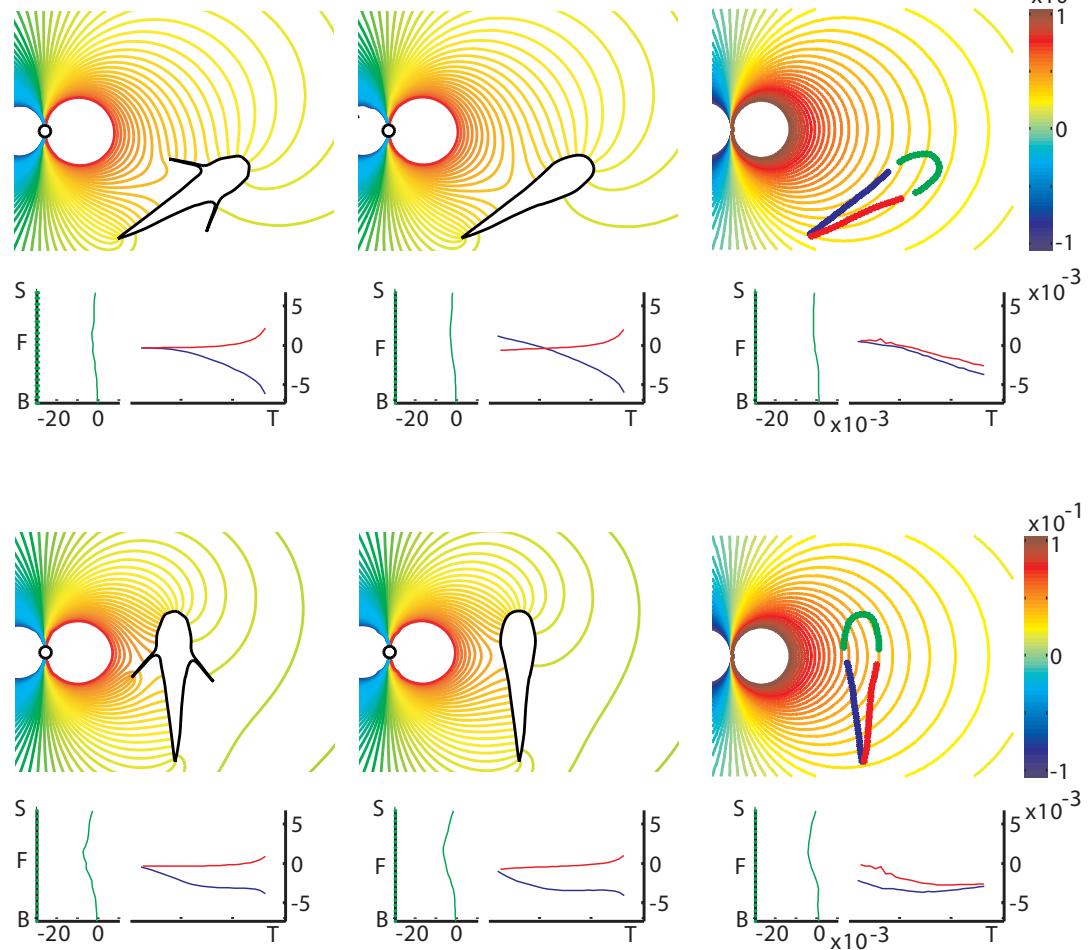
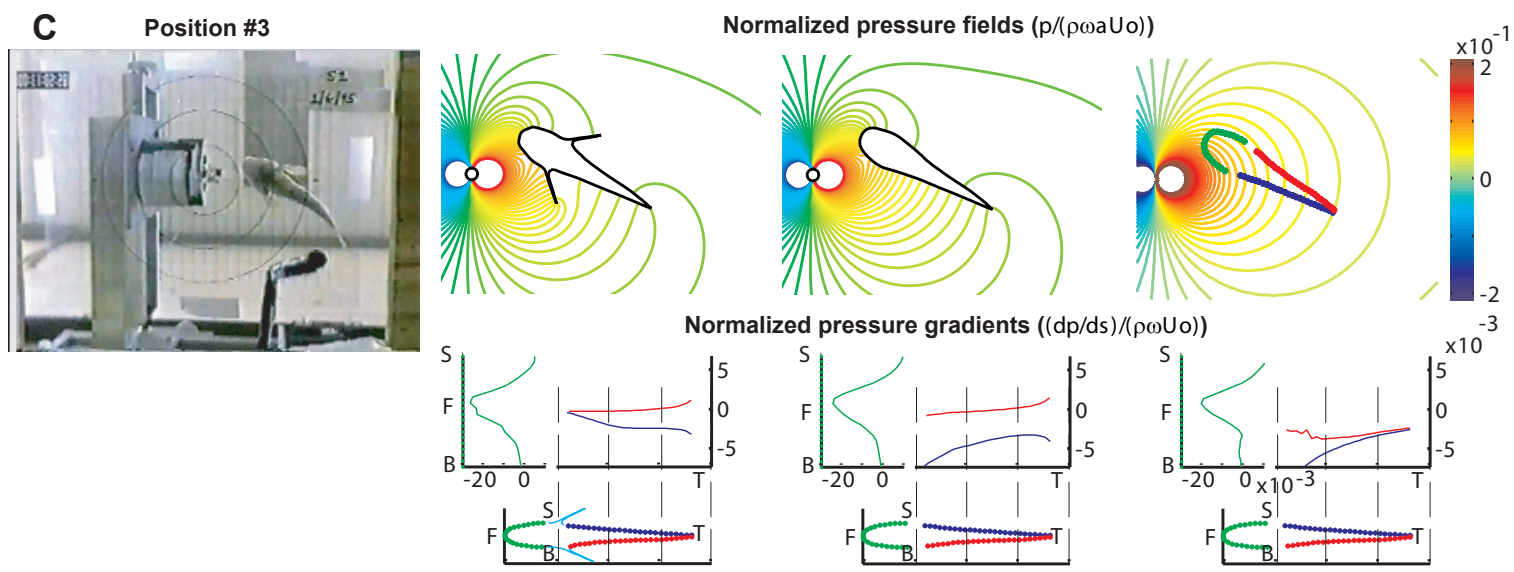

Figure 4-6: Experimental set up and video stills from a strike sequence (see text). The video camera was placed below the holding tank, aiming up through to see the bottom of the sculpin. Circles are of radius $3 \mathrm{~cm}, 6 \mathrm{~cm}$, and $9 \mathrm{~cm}$, with the sphere located at the center and oscillating in a direction parallel to axis that extends through the center of the mini shaker (the x-axis of the tank in this picture). (AC) The normalized pressure fields (top row) and corresponding pressure differences along the lateral line (bottom row) are shown for three strike positions. (2nd \& 3rd columns.) Numerical results with body present, without/with fins respectively. (4th column) Calculated results without body present. 


\section{Sculpin experiment}
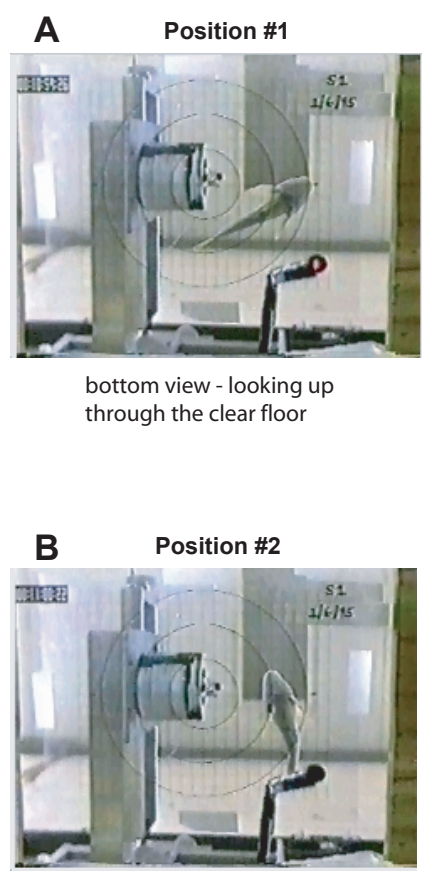

3D numerical simulations
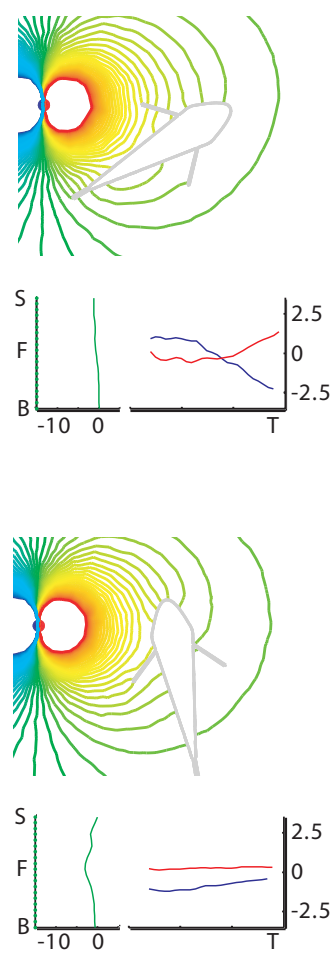
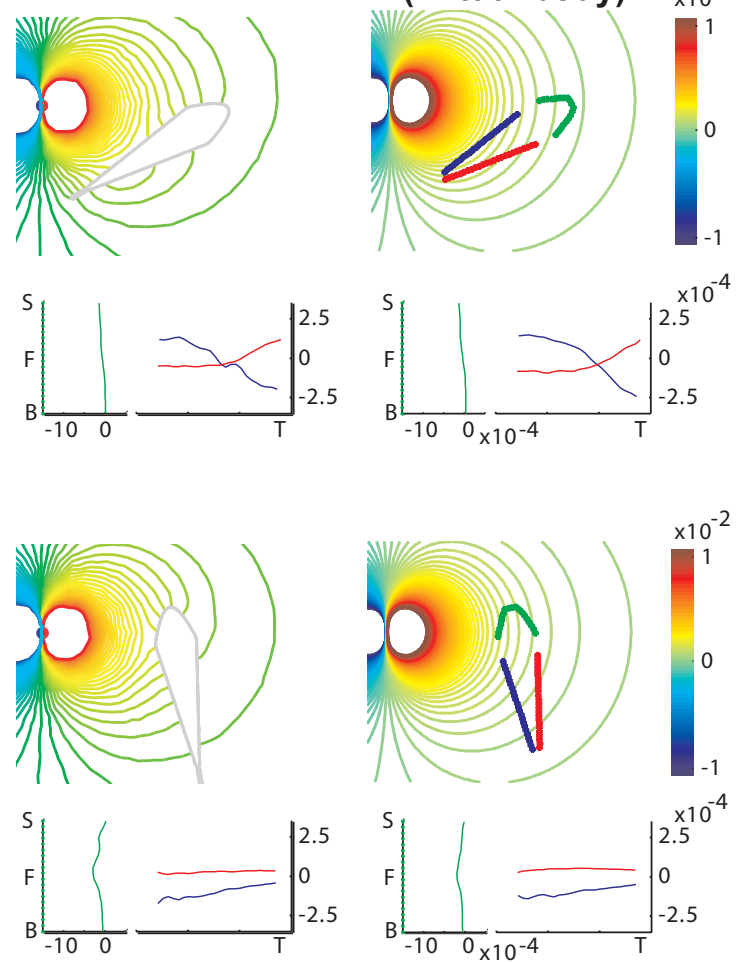
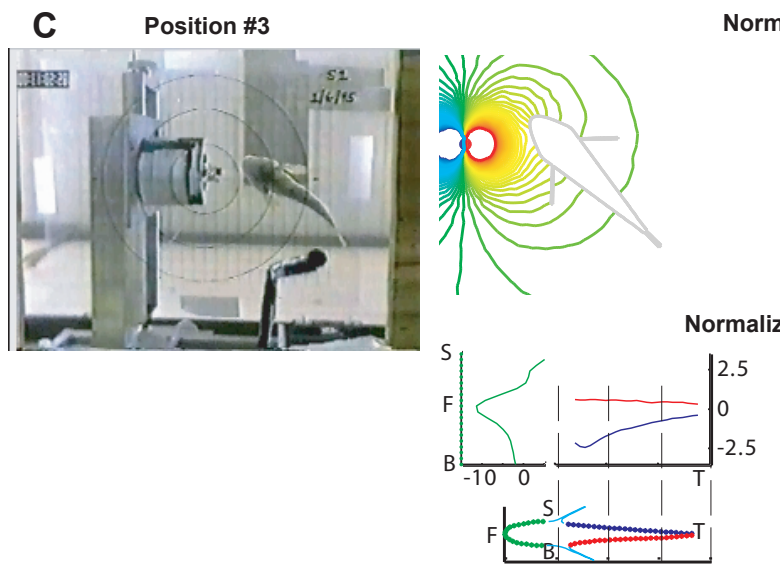

Normalized pressure fields ( $p /(\rho \omega a \cup o))$
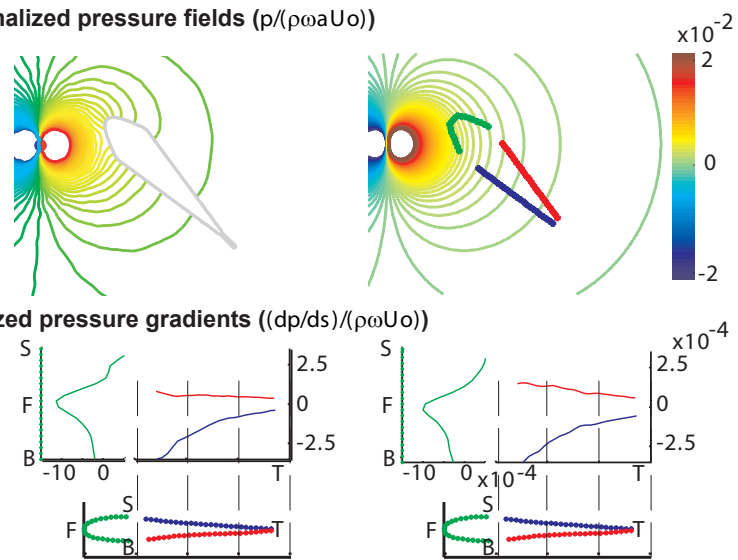

Figure 4-7: A two-dimensional slice through the three-dimensional mid-plane of the sculpin. Compare the 3D signal and body effects with the 2D results presented in figure 5. Descriptions and set up are given in figure 4-6. Experimental set up and video stills from a strike sequence (see text). 


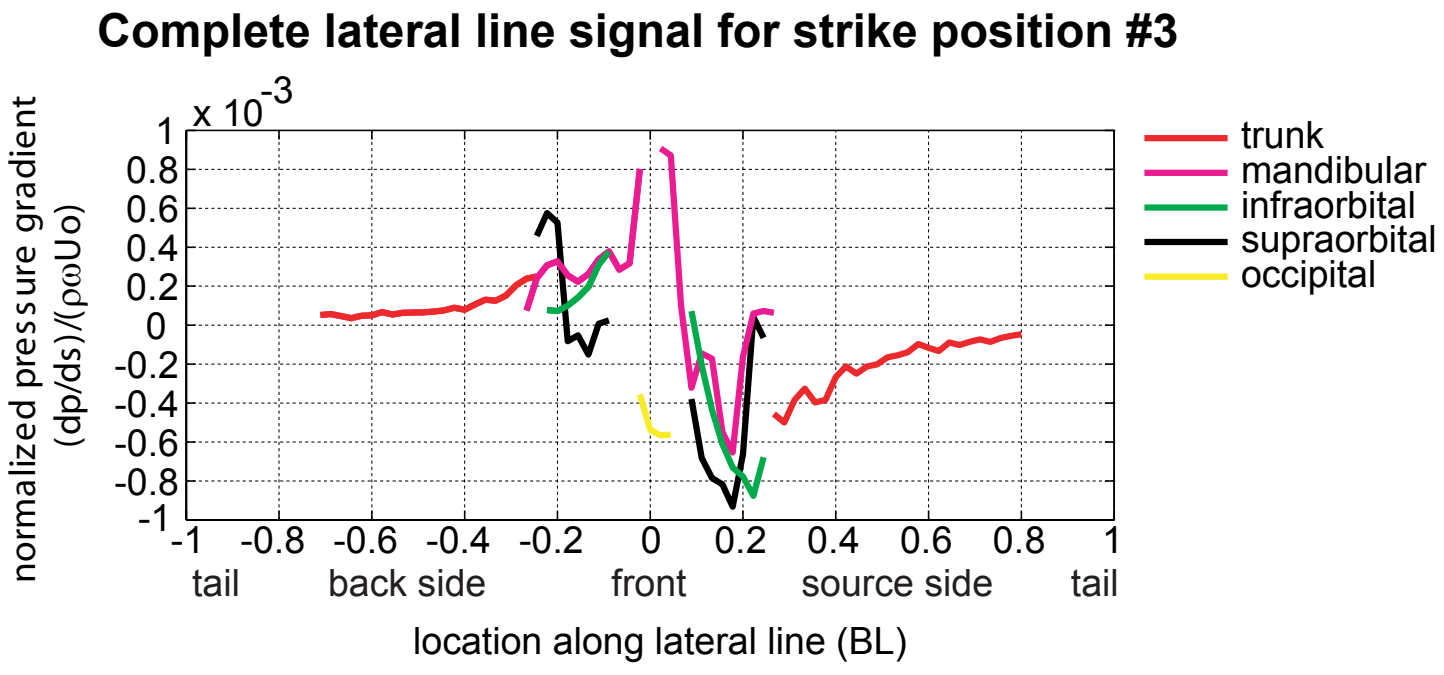

Figure 4-8: The received dipole signal to the whole canal lateral line system for strike position 3. The "heartbeat" signal is distinguishable, especially in the mandibular portions. 
Pressure contours around the surface of the 3D sculpin are presented for the body with fins extended, and with fins retracted cases.

The most obvious result by inspection of the pressure contour plots is that the presence of extended fins does not cause a significant interruption to the hydrodynamic field for the oscillating sphere in these locations. This is confirmed in the pressure gradient plots, where there is little distinction between the extended/retracted fin cases (see overlap of magenta \& cyan lines in figure 4-9). The placement of the lateral line above the pectoral fins seems to allow for clear dipole signal reception in these cases.

In these canonical cases, the spatial gradient patterns seen in figure 4-3C can be readily identified. When the dipole source is oscillating parallel to the longitudinal axis of the fish, the pressure difference pattern formed on the source side lateral line matches the predicted shape (figure 4-3, 1st col). The peak amplitude of the typical "hat" pressure gradient profile occurs at the same position on the lateral line, in line with the actual lateral location of the source. For cases A and B, the zero crossings fall neatly within the confines of the tail portion of the lateral line. The zero crossings predict distances of $0.20 \mathrm{BL}$ and $0.31 \mathrm{BL}$, respectively, which is close to the actual distances of $0.19 \mathrm{BL}$, and $0.29 \mathrm{BL}$, respectively. For case $\mathrm{C}$, the sphere is far enough away from the fish that the zero crossings begin on the source side and wrap part way around the front side. This distorts the natural signal, and predicts a distance of $0.37 \mathrm{BL}$, when the actual distance is $0.49 \mathrm{BL}$.

When the sphere oscillates in a direction perpendicular to the long axis of the fish, the pressure gradient pattern has the predicted anti-symmetric pattern (figure $4-3,2 \mathrm{nd}$ col). The location of the zero crossing correctly predicts the perpendicular bisector formed with the sphere in cases $\mathrm{D}$ and $\mathrm{E}$, but in case $\mathrm{F}$ the received dipole signal is distorted as it hits the contours of the nose of the fish, giving 3 zero crossings within a short span of the right lateral line. When the sphere is overhead (case D), the presence of the dorsal fin reduces the peak pressure difference. The predicted distance in this case is $0.26 \mathrm{BL}$, which is still close to the actual value of $0.29 \mathrm{BL}$. When the sphere is close to the side of the fish (case E), the predicted distance is 
0.30 BL, which is very close to the actual value of $0.29 \mathrm{BL}$. When the sphere is in the furthest location (case F), the fish could choose to use the undistorted half of the signal since a zero crossing and one peak still provides useful information when the sphere oscillates perpendicular to the lateral line. The predicted distance is $0.44 \mathrm{BL}$, which is not too far from the actual distance of 0.5 BL.

Consistent with conclusions drawn earlier, the flow around fin insertion points, pectoral, dorsal affects the proximate area, but not the received dipole signal to the lateral line system. However, it is also clear that as the source moves farther from the body, not only does the amplitude of the received dipole signal decrease, but it also becomes distorted as the length of any available lateral line section runs short.

\subsubsection{Canonical cases: the signal to the superficial lateral line system}

Figure 4-10 presents an instantaneous snapshot of the normalized velocity vectors at the cell centroids adjacent to the fish body. The location of the sphere is $(0.56,0.33$, 0.11) BL (body lengths), identical to cases $\mathrm{B}$ and $\mathrm{E}$ of figure 4-9, though the time has advanced $1 / 4$ of an oscillation period. The coordinate system has its origin at the nose of the sculpin, located $0.12 \mathrm{BL}$ above the floor. When looking down on the fish, the nose points North, the tail points South, the right lateral side points East, and the left lateral side points West. The $\mathrm{x}$-axis is aligned with the rostrocaudal axis of the fish, with positive in the direction of the tail. The y-axis is aligned with the lateral axis of the fish, with positive toward the right side of the fish. The z-axis is aligned with the dorsoventral axis of the fish, with positive toward the dorsal side of the fish. The floor plane is parallel to the xy-plane.

With the coarser grid resolution (i.e. $2 \mathrm{~mm}$ edges), the tetrahedral cell centroids are found at a heights of about 0.5-0.8 $\mathrm{mm}$ above the fish body. As mentioned earlier, this has almost no effect on the pressure or pressure gradient resolution at the body surface, but it does prevent resolution of the boundary layer flow. The velocity values calculated at this resolution are essentially potential in nature, and can be considered 


\section{Pressure and pressure gradient comparison for pectoral fins extended $(-)$ vs. retracted $\left({ }^{-}\right)$cases}

A
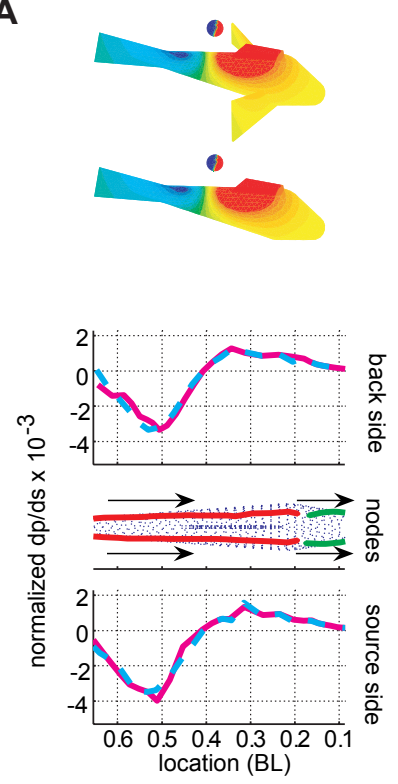

D

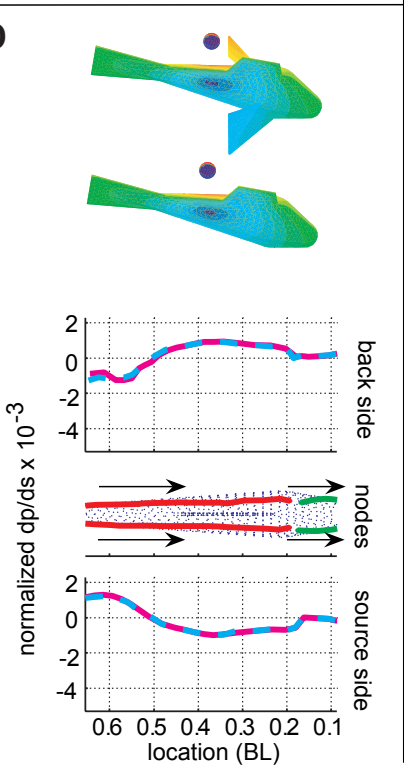

B
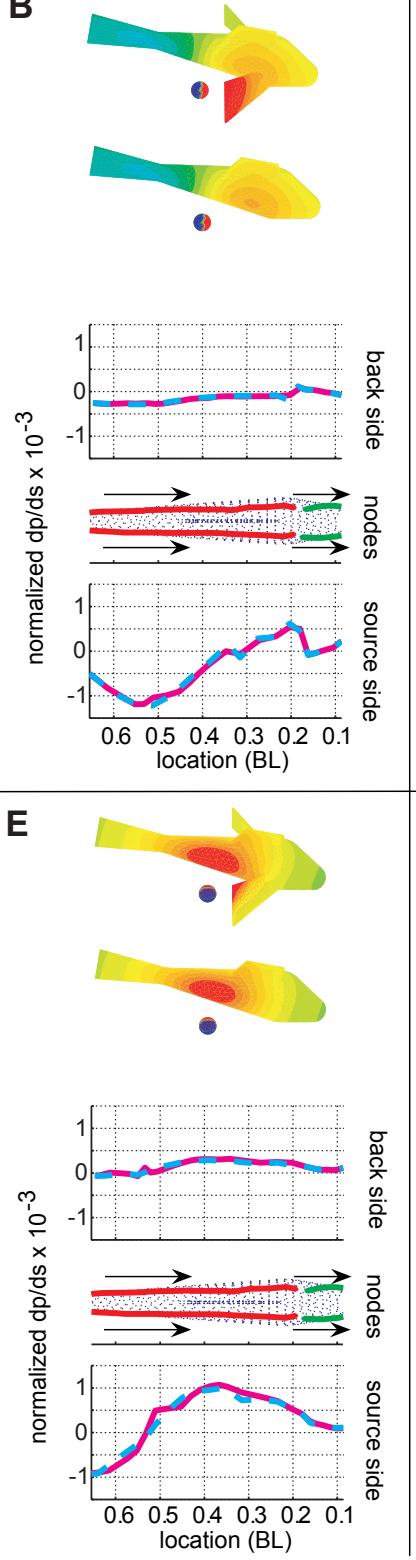

C
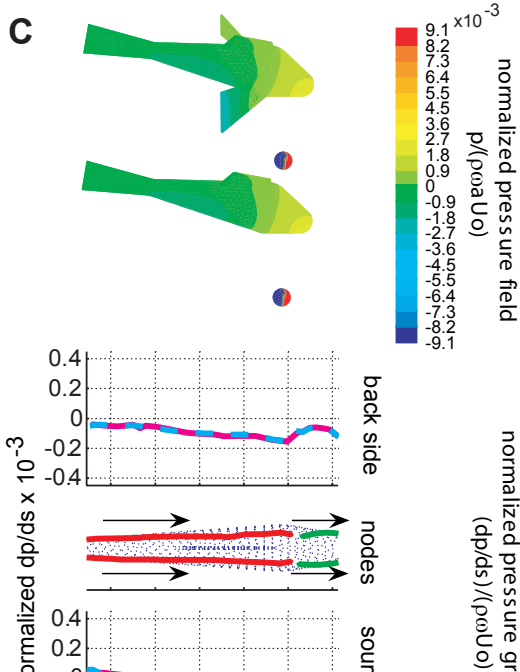

产

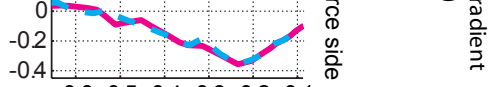

0.60 .50 .403020 .1 location $(\mathrm{BL})$

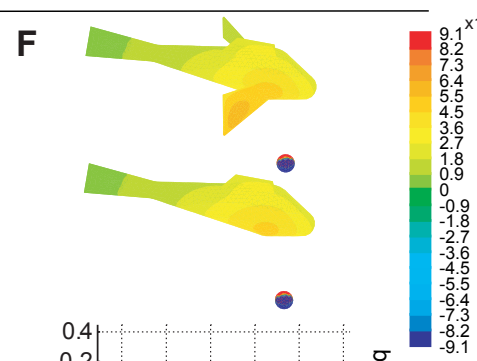

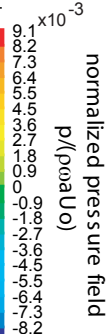

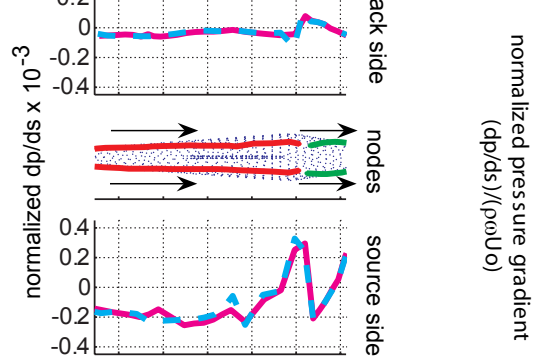

0.50 .40 .30 .2
location $(\mathrm{BL})$

Figure 4-9: An instantaneous snapshot of the pressure field on the body surface, and the resulting pressure gradient to the canal lateral line, are shown for the cases of a sphere oscillating parallel and perpendicular to a sculpin. In cases $\mathrm{A}$ and $\mathrm{D}$, the sphere is located at $(0.56,0,0.33) \mathrm{BL}$ (body lengths). For cases B and E, the sphere is located at $(0.56,0.33,0.11) \mathrm{BL}$. In cases $\mathrm{C}$ and $\mathrm{F}$, the sphere is located at $(0.22$, $0.56,0.11) \mathrm{BL}$. The coordinate system has origin is on the nose, the first coordinate is toward the tail along the rostrocaudal axis, the second is to the right along the lateral axis, and the third is up along the dorsoventral axis. Lateral line nodes (canal pore openings) are approximately spaced $2 \mathrm{~mm}$ apart. 
the $U_{\infty}$ values influencing the boundary layer velocity profiles at the fish surface.

Since the superficial neuromasts have a best sensitivity in one oscillation direction, which is unique to each neuromast and unknown apriori, two arbitrary directions were chosen. One direction is positive when deflected towards the tail, and one direction is positive when defected toward the top of the fish. The magnitude of the flow velocity component in the direction of sensitivity is shown in figures $4-10 \mathrm{C}, \mathrm{D}, \mathrm{G}, \mathrm{H}$, with a negative value indicating the flow component is opposite the direction given by the black arrows.

The flow patterns around the fish show clearly reveal whether the sphere is oscillating parallel or perpendicular to its side. When oscillating parallel to its side, the flow has two nodes where the flow either leaves in all directions or returns from all directions. In figure $4-10 \mathrm{~A}, \mathrm{~B}$, one of the those nodes is located at the pectoral fin insertion point, and the other near the tail. When the sphere is oscillating perpendicular to the side of the fish (figure 4-10E,F), there is one node, from which flow spreads out in all directions. Qualitatively, it appears that only the flow located directly at fin insertion points is significantly altered.

Quantitative results at superficial neuromasts located along the canal lateral line system confirm there is no discernable effect of the fins presence in altering the received dipole signal (figure 4-10C,D,G,H). Dipole signals to the superficial lateral line are quite different depending on their preferred direction of sensitivity. When the directional sensitivity is in line with the axis of oscillation, the patterns to the superficial lateral line are of the "Mexican hat" shape (figure 4-10C,H), whereas when the directional sensitivity is opposite the axis of oscillation, the patterns are of the "heartbeat" shape (figure 4-10D,G). Therefore the peak locations of approximately $0.53 \mathrm{BL}$ in figure $4-10 \mathrm{C}$ and $0.48 \mathrm{BL}$ in figure $4-10 \mathrm{H}$ are close to the actual location of 0.5 BL. Likewise, zero crossings of approximately $0.55 \mathrm{BL}$ in figure $4-10 \mathrm{D}$ and 0.5 $\mathrm{BL}$ in figure $4-10 \mathrm{G}$ are also close (or exact). The peaks and zero crossings on the back side of the body are further shifted away from the actual location of the sphere. 

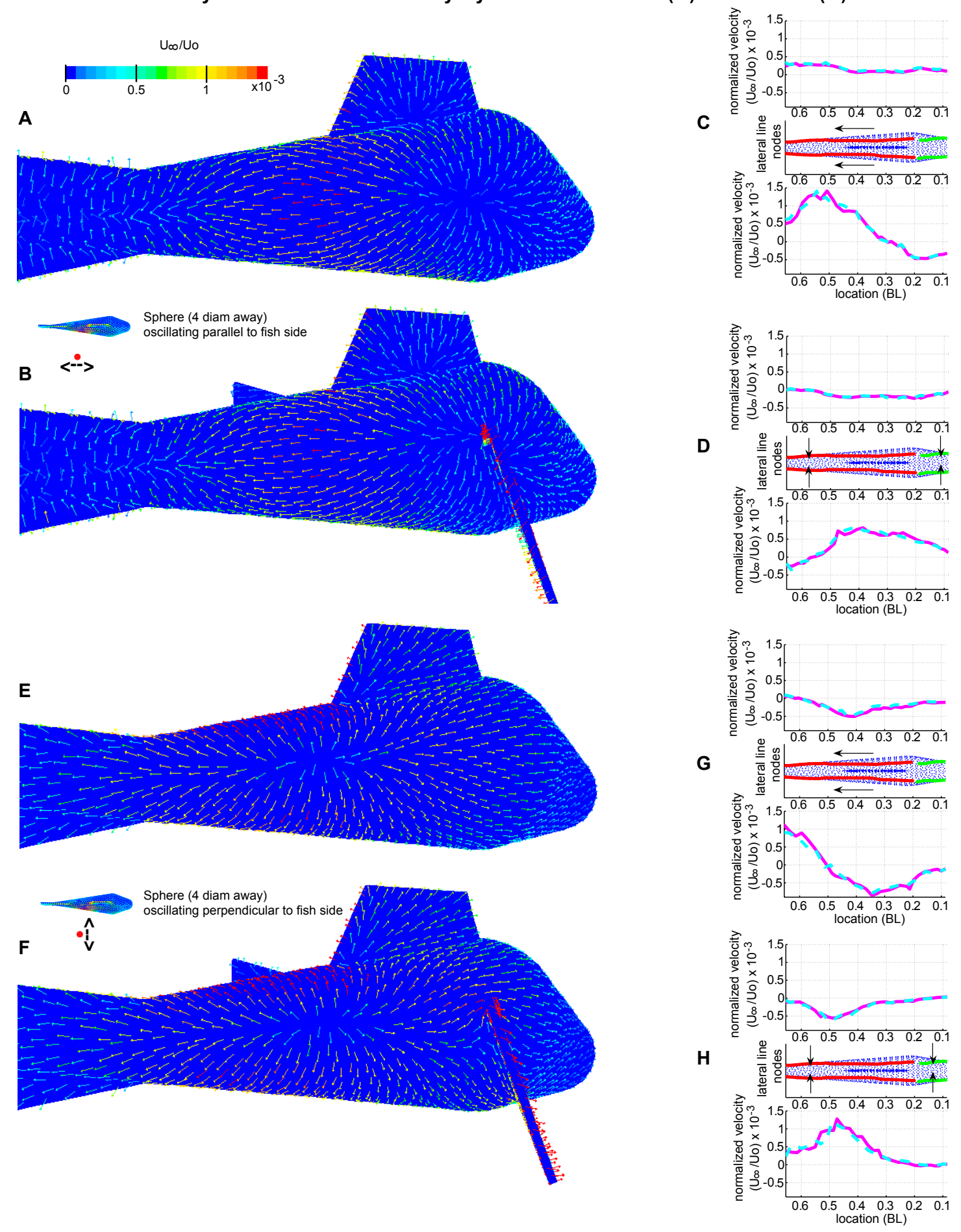

Figure 4-10: An instantaneous snapshot of the normalized velocity vectors at the cell centroids adjacent to the fish body. The location of the sphere is $(0.56,0.33$, 0.11) BL, identical to cases $\mathrm{B}$ and $\mathrm{E}$ of figure 8, though the time has advanced $1 / 4$ of an oscillation period. The sphere is oscillating parallel to the fish (A) with fins extended, and (B) retracted, and it is oscillating perpendicular to the fish (E) with fins extended, and $(\mathrm{F})$ retracted. The black arrows show the positive direction of neuromast polarization. 


\subsubsection{The signal to the lateral line system for a sphere oscil- lating in the dorsoventral direction}

Other cases involve a sphere oscillating in the dorsoventral direction to the side of a sculpin, and results are presented in figures 4-11-4-13. The signal to the canal lateral line system is the pressure gradient. Plots A and B show the normalized pressure contours around the body surface and plot $\mathrm{C}$ compares the normalized pressure gradient for the fins extended/retracted cases. The signal to the superficial lateral line is velocity in the direction of neuromast polarization. Plots D and E show the normalized velocity field outside the boundary layer over the body surface, and plots $\mathrm{F}$ and G show the stimulus to the neuromast when the direction of polarization is toward the nose and top of the fish respectively. All plots are at the instant of time that the pressure and velocity are at a maximum. In this case, the time is delayed by $1 / 4$ of a period for the velocity.

When the sphere oscillates in the dorsoventral direction, there is almost no stimulation to either the canal or superficial lateral line on the back side of the fish. On the source side of the body, superficial neuromasts which have their axis of polarity in alignment with the sphere oscillation axis are maximally stimulated, and likewise for portions of the canal lateral line which are in alignment (such as portions of the infraorbital, supraorbital, and mandibular lateral line). This is especially the case when the sphere is near the front of the fish.

Sometimes there can be ambiguity about the location of the dipole source. Figures 4-12 and 4-13 show that when the sphere moves further away from the side of the fish, the magnitude decreases but the overall pressure gradient patterns and velocity gradient patterns remain very similar. This is the case because the lateral line system is not sampling the spatial field in a direction of maximal change (in alignment with the axis of oscillation), but instead spatially samples the dipole field in the direction of least change (in the plane perpendicular to the oscillation axis, and running through the center point of the sphere). 
Pressure and pressure gradient comparison for pectoral fins extended $(-)$ vs. retracted $\left(-{ }^{-}\right)$cases

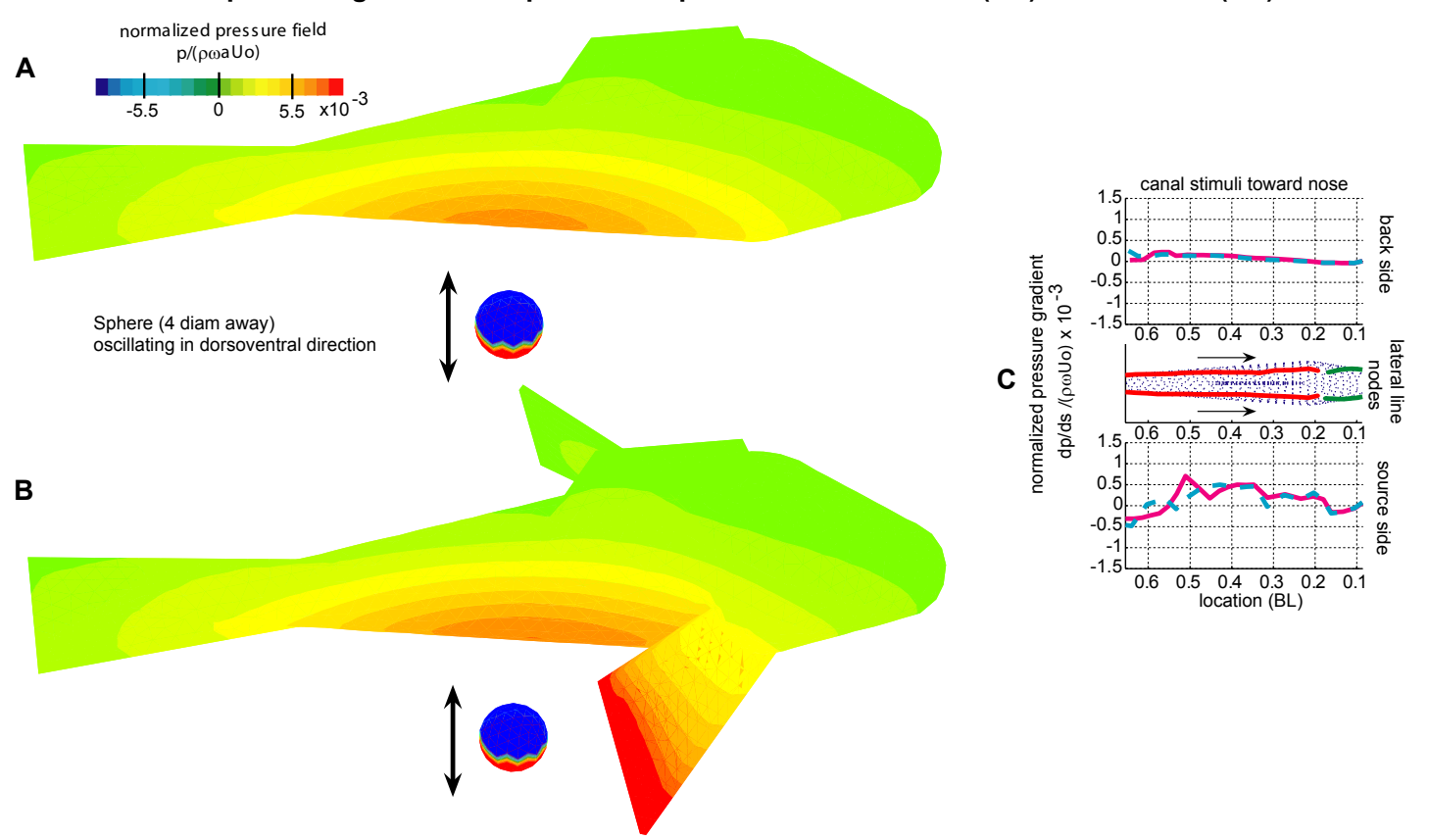

Normalized velocity field outside the boundary layer for fins extended $(\rightarrow$ vs. retracted $(-)$ cases
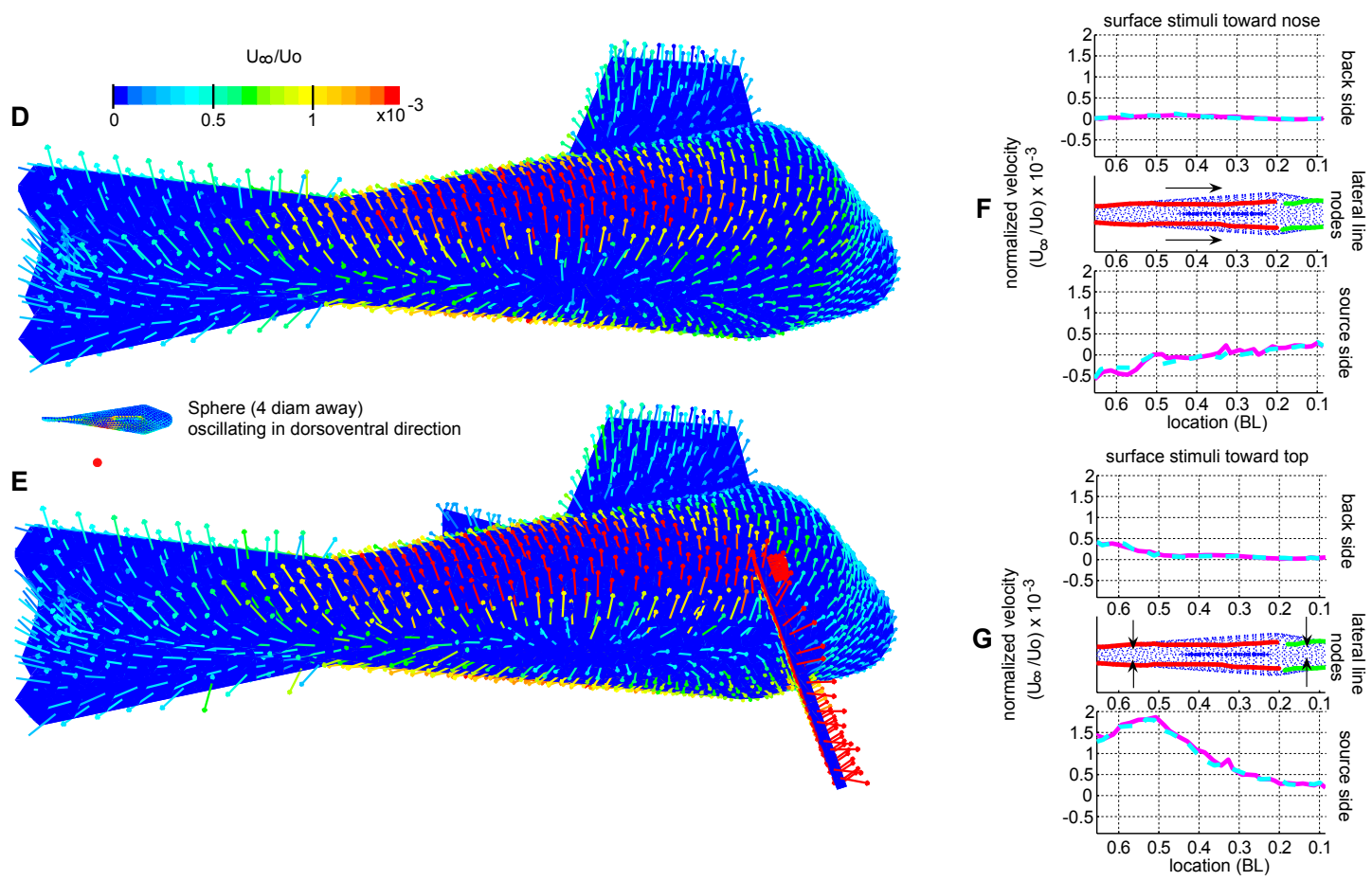

Figure 4-11: Instantaneous snapshots of the $(\mathrm{A}, \mathrm{B})$ pressure and $(\mathrm{D}, \mathrm{E})$ velocity fields on the body surface and adjacent cell centroids are shown for a sphere oscillating in the dorsoventral direction next to the sculpin. The velocity is shown at $1 / 4$ of an oscillation period later than the pressure. The sphere is located at $(0.56,0.33,0.11)$ BL. (C) The normalized pressure gradient along the lateral line. (F,G) Component of normalized surface velocity in alignment with superficial neuromast, with black arrows showing direction of positive polarity. 
Pressure and pressure gradient comparison for pectoral fins extended $(-)$ vs. retracted $(--)$ cases

A normalized pressure field
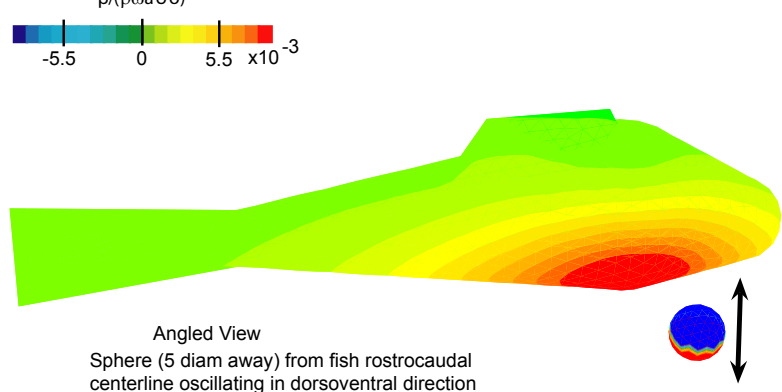

centerline oscllating in dorsoventral direction

B

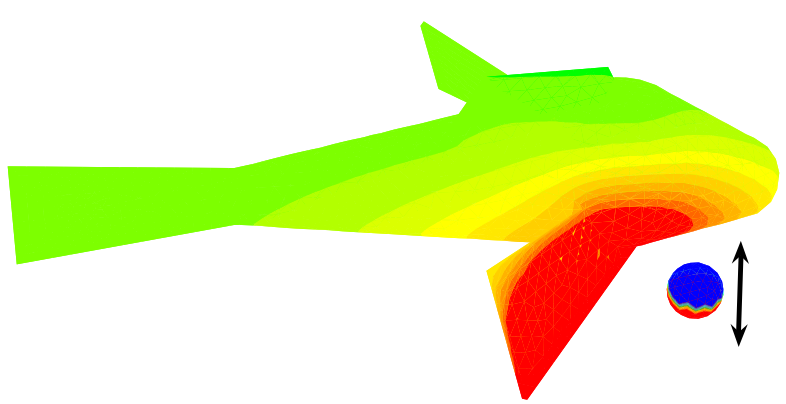

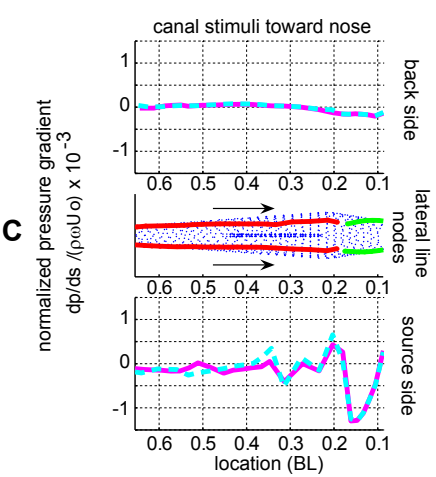

Normalized velocity field outside the boundary layer for fins extended $(\rightarrow$ vs. retracted $(-)$ cases
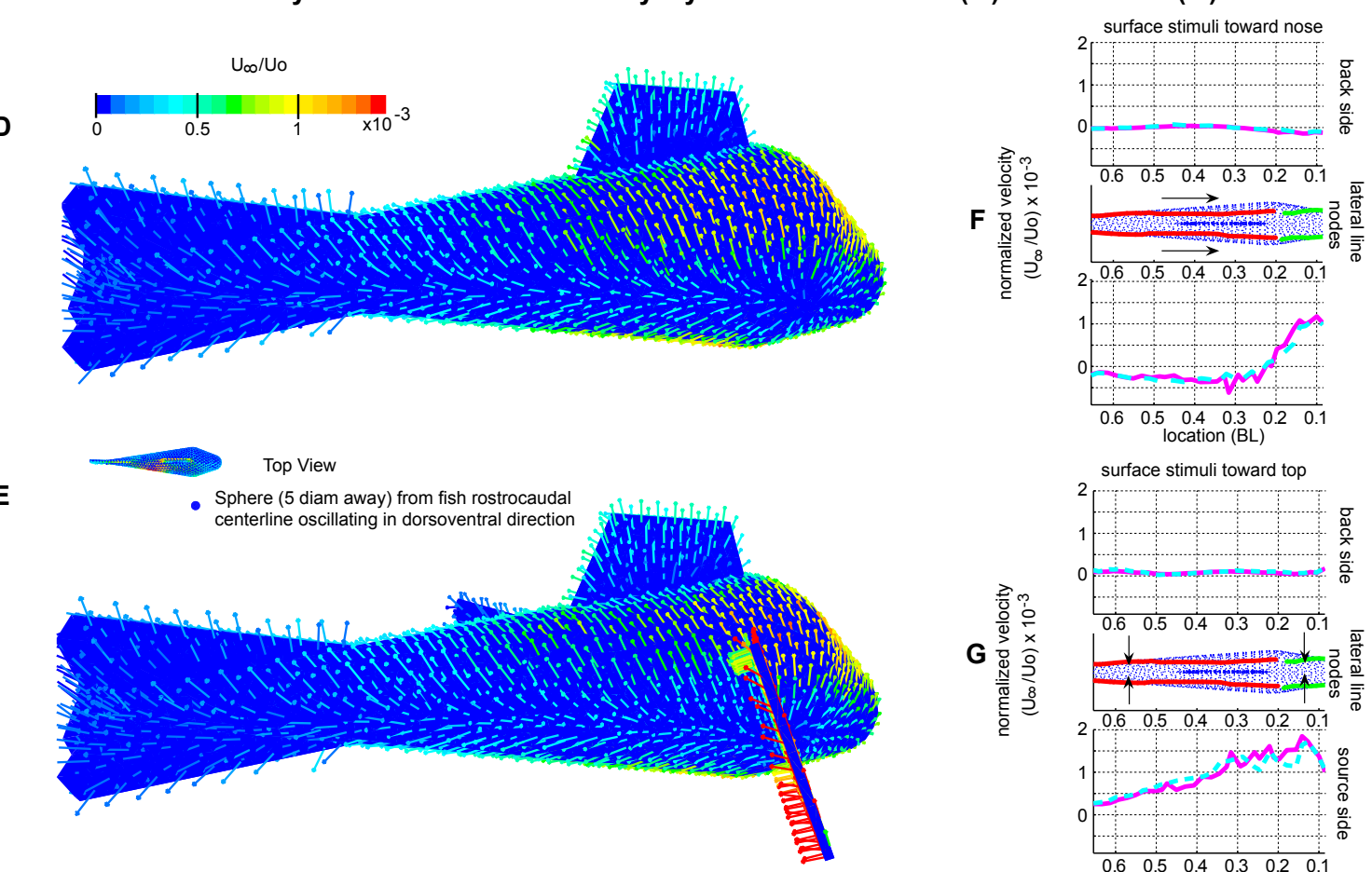

Figure 4-12: Description as given in figure 4-11. The sphere is located at $(0.22,0.33$, $0.11) \mathrm{BL}$ 


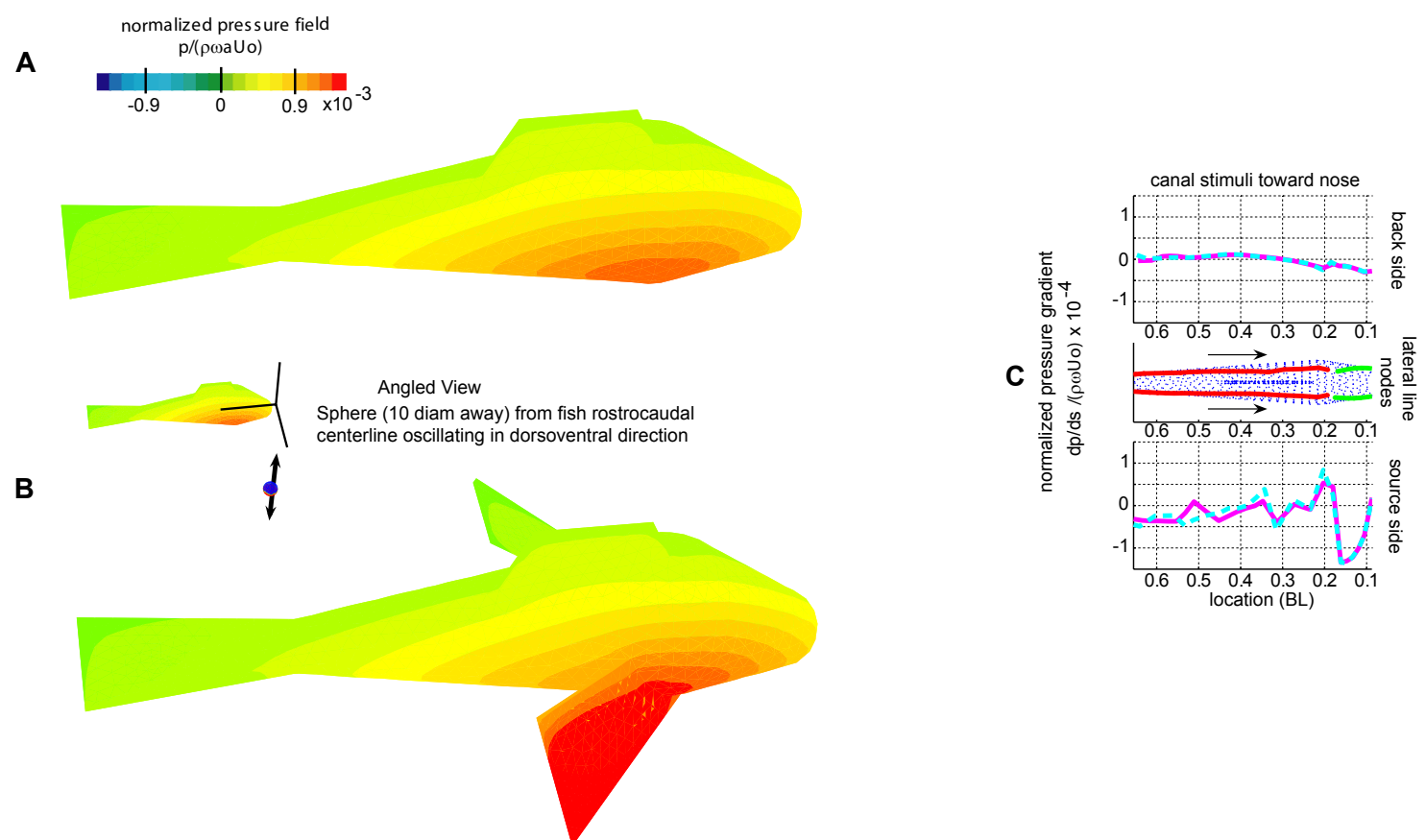

Normalized velocity field outside the boundary layer for fins extended $(\rightarrow$ vs. retracted $(-)$ cases

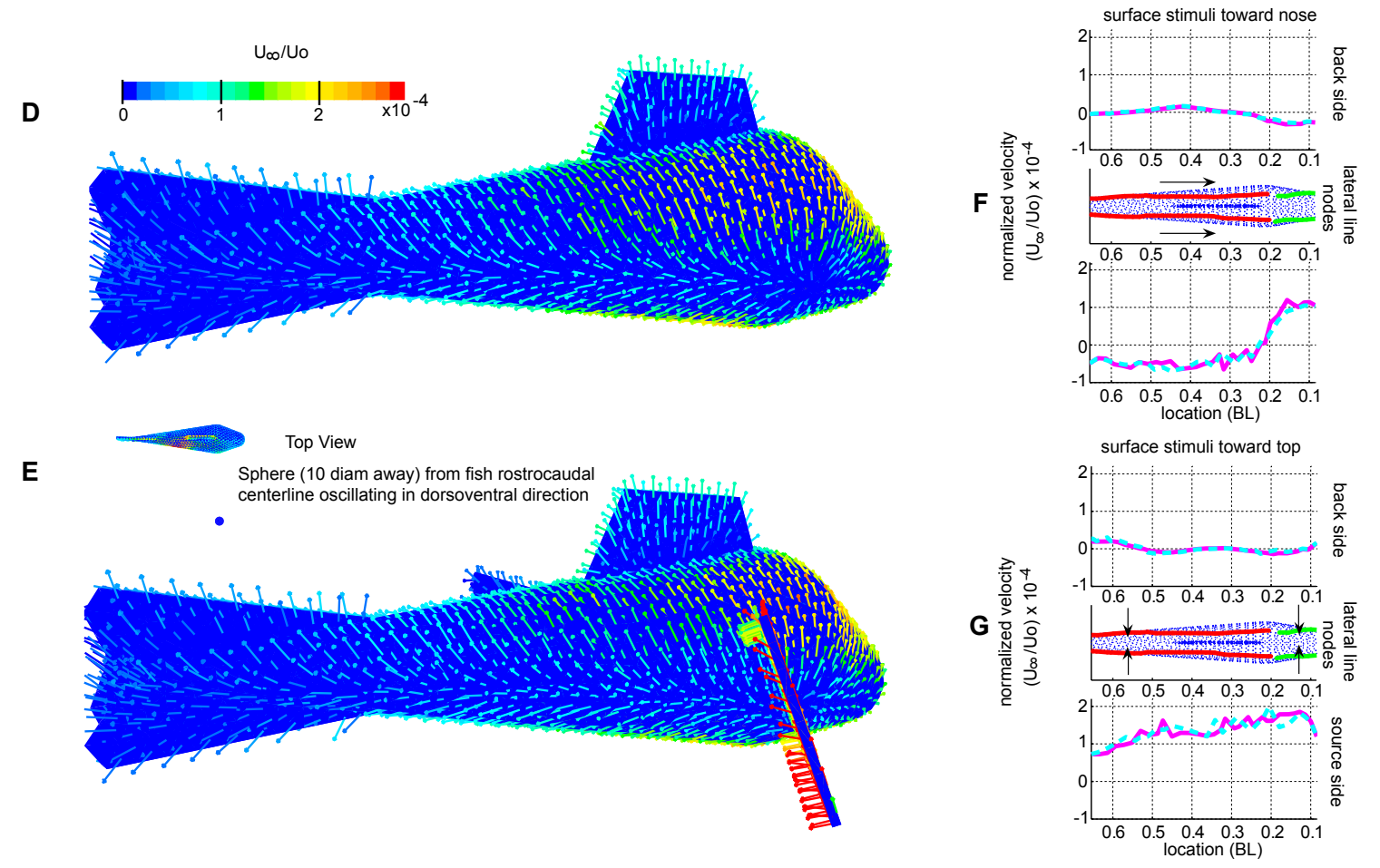

Figure 4-13: Description as given in figure 4-11. The sphere is located at $(0.22,0.67$, $0.11)$ BL. Note that the scales have changed from figure 4-12, being approximately an order of magnitude less. 


\subsubsection{Predictions of surface flow on a virtual body using po- tential flow theory}

Previously (chapter 2) it was shown that for a dipole potential next to a wall, the pressure at the wall doubles relative to the pressure at the same location in an unbounded medium. In many cases, the results have shown that using potential flow theory to predict pressures at some locations on a virtual fish body also works as a first approximation when the pressure is doubled. It may also be asked whether potential flow theory can be used to approximately predict the tangential flow over the surface of a virtual body, when the value is doubled relative to the same location in an unbounded medium. The normal velocity is of course zero at the fish surface.

The process is not as simple as predicting the pressure, because velocity at any particular location is a vector, whereas pressure is a scalar. The velocity vector is determined by taking the gradient of the velocity potential, and then doubling the value to approximate the presence of the body at that location. The surface tangent vector can be determined by projecting the velocity vector onto a plane perpendicular to the surface normal vector. This can also be determined by taking the cross product of the normal and velocity vectors, with the cross product of the normal vector a second time. To approximate the presence of the wall, the magnitude is doubled.

$$
\left.\vec{v}\right|_{\text {wall }}=2\left(\vec{t} \cdot \vec{v}_{\mathrm{sph}}\right)=2\left(\vec{n} \times \vec{v}_{\mathrm{sph}}\right) \times \vec{n}
$$

The 3D results for a sphere oscillating parallel and perpendicular to the side of a sculpin are presented in figure 4-14. The case is identical to that found in figure 4-10, but now potential flow results are presented in addition to the numerical results. Since it is hard to visually inspect differences in vector angle, the difference in angle and magnitude between numerical simulation and potential flow theory predicted vectors are quantized and displayed graphically using surface contour plots.

In comparing the streamlined with pectoral fins extended numerical simulation cases, there is visual agreement over almost the entire body surface. At the pectoral fin insertion point, there is a difference in the flow with the streamlined case because 
the flow is impeded by the presence of the fin. When the sphere is oscillating in such a way that a node appears at the pectoral fin insertion point (figure 4-14 A,B), there is very little interference to the flow by the extended fin. However, in what will be the majority of cases, where the sphere is oscillating in such a way that there should be a directed flow at the pectoral fin insertion point, the flow must be altered to go around the fin (figure 4-14 C,D). The flow region affected by the fins, due to the dipole field of an oscillating sphere, is small. This can be confirmed by looking for differences between the streamlined and fin extended cases in the angle and magnitude error contour plots. Using the potential flow theory approximation, every point on the body is treated independently of all others, and so the presence or absence of fins should make no difference in the calculations. Therefore, major differences are due to differences with the numerical simulation cases, highlighting the region of altered flow.

Using the potential flow theory approximation to predict the flow around the body surface seems to work remarkably well over much of the surface. There are significant errors at corners and insertion points as to be expected. However, there is also significant error when the flow is directed directly into the body. These are nodes in the flow field, where in the numerical simulations the flow can be effectively halted and affected by the surrounding fluid. In the potential flow theory approximation, these node locations do not show the effects of halted tangential flow (of course the normal flow is forced to be zero by definition), and are significantly wrong both in direction and magnitude. It should be noted that the flow is already much reduced at these points, so in absolute terms, the error is likely of little significance compared to absolute errors seen around the rest of the body.

The potential flow theory approximation would not be expected to be able to predict flow around the back side of the body, since the body should block the flow. What little it might predict, it would be expected to do better when the source is further away since the finite size of the body will a much smaller portion of the expanding dipole flow field volume. However, the flow direction along the back side is surprisingly well predicted (excluding node locations of course) when the axis of the 
oscillating sphere is parallel with long axis of the body, and less well when perpendicular. In the first case, the flow is naturally more aligned with the body surface, while in the second case the flow is trying to push through the body, which has already been shown to be difficult to predict well. In all cases, the magnitude along the back side is not well predicted.

\subsubsection{Validation of the strain rate predictions on the fish surface}

The approximations used to determine the strain rate at the wall due to flow induced by a nearby oscillating sphere were validated at points of maximal tangential velocity along the wall. However, the value of this approximation extends over a more significant region than just a few points.

Figure 4-15 shows the results using the velocity outside of the boundary layer, along with equation 4.7 , for predicting the surface strain rate. For one case, all fins on the sculpin were removed, allowing for the geometric generation of a resolved boundary layer around the surface of the fish. A $3 \mathrm{~mm}$ sphere was located at a distance of $5.5 \mathrm{~cm}$ from the midline of the fish, and roughly at what would normally be the pectoral fin insertion point. The sphere was oscillated perpendicular to the side of the fish, at $50 \mathrm{~Hz}$, with a velocity amplitude of $7 \mathrm{~mm} / \mathrm{s}$.

Plots A-C used the resolved boundary layer mesh when solving for the induced flow around the fish surface. Plot D uses the coarse meshing used on all other 3D fish bodies, with nodes spaced approximately $2 \mathrm{~mm}$ apart. Plot A shows the strain rate at the surface of the fish, as calculated using equation 4.4. This is the benchmark strain rate. Plots $\mathrm{B}$ and $\mathrm{C}$ use the velocity at $1 \mathrm{~mm}$ and $2 \mathrm{~mm}$ above the surface to predict the strain rate below equation 4.4. Finally, plot D shows the same prediction from $2 \mathrm{~mm}$ away, but for using velocities obtained from a coarse grid solution to the flow.

The main result is that when the flow is accurately calculated, equation 4.4 does an excellent job of predicting the surface strain rate. For experiments that use Digitial 

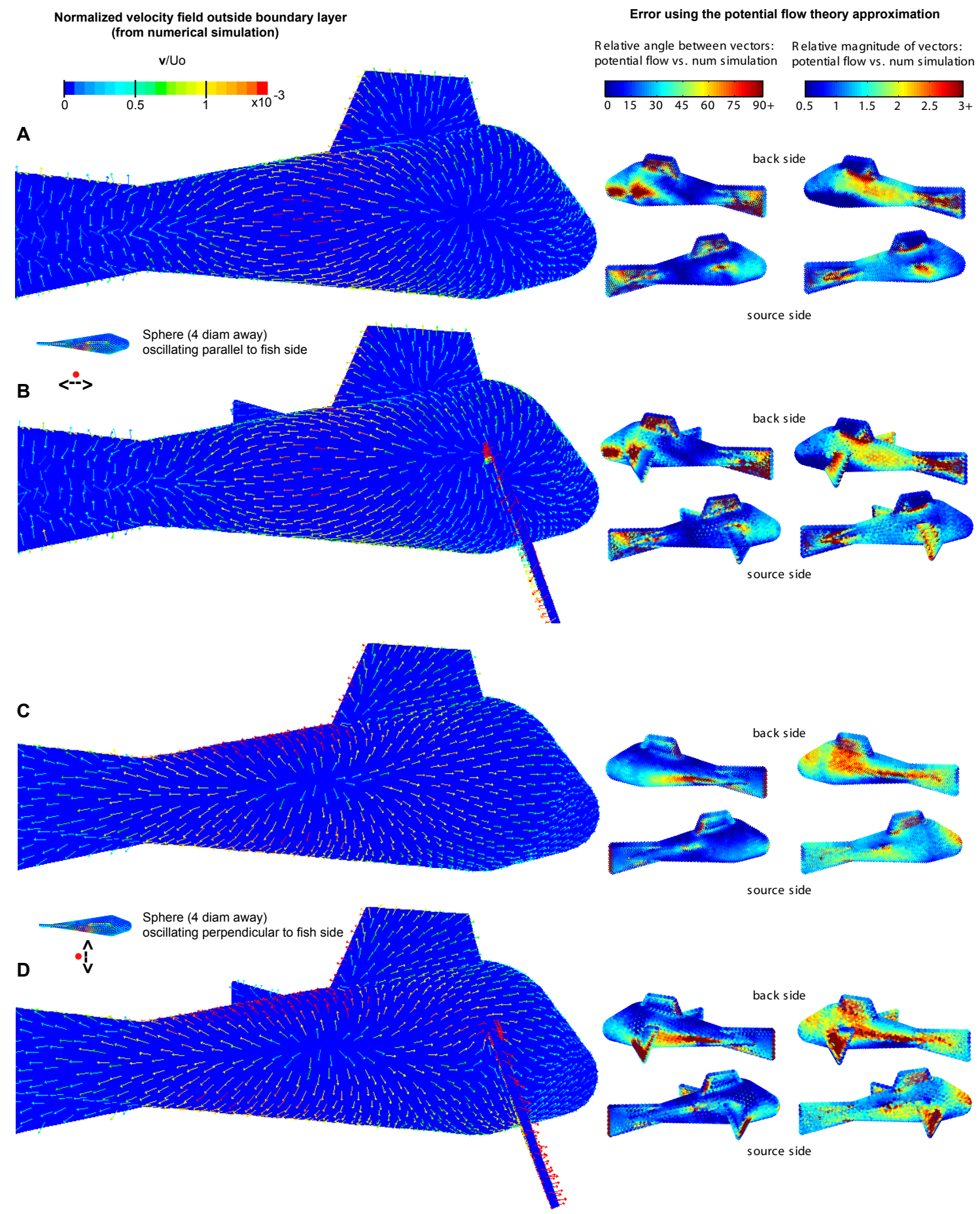

Figure 4-14: Numerical simulations of flow outside the boundary layer of the fish, induced by a nearby oscillating sphere, are shown in plots on the left (see figure 4$10 \mathrm{~A}, \mathrm{~B}, \mathrm{E}, \mathrm{F}$ for further explanation). Plots on the right show the relative errors in vector angle and magnitude between potential flow predictions and numerical results. 
Particle Image Velocimetry (DPIV) to resolve an oscillating flow around a fish, this equation will allow for the determination of the shear stress, which is just $\mu S_{\text {wall }}$. Likewise, figure 4-15 shows that an accurate "potential flow" solution still provides an adequate prediction of the surface strain rate over much of the body of the fish.

\subsubsection{Applying results to experiments}

In one study, Blind Mexian Cave Fish whose canal neuromasts had been destroyed where still able to detect the presence of an oscillating sphere at a distance of $20 \mathrm{~cm}$ away (Abdel-Latif et al., 1990). In the results presented, the sphere had a diameter of $5 \mathrm{~mm}$, used displacement amplitudes of 0.2-1.4 mm, and oscillated at $50 \mathrm{~Hz}$ and $70 \mathrm{~Hz}$. The conclusion drawn was that the superficial neuromasts are all that is needed for this task of prey detection and localization. However, this would indicate an extremely sensitive detection threshold, as will be demonstrated, indicating there are likely other mechanisms at work.

In another study, blinded Lake Michigan Mottled Sculpin whose superficial neuromasts had been damaged by skin abrasion where able to detect the presence of a nearby $(3-6 \mathrm{~cm})$ oscillating sphere (Coombs et al., 2001). However, when the canal neuromasts where damaged (by treating with a solution of gentimicin), the fish response rates decreased to spontaneous levels. The sphere had a diameter of $6 \mathrm{~mm}$, was oscillated at frequencies of $10 \mathrm{~Hz}$ and $50 \mathrm{~Hz}$, and had source peak-to-peak velocities of $0.11-0.18 \mathrm{~m} / \mathrm{s}$. The study concluded that it was the canal neuromasts which mediated the fish's response to the stimulus.

At issue in both of these studies is the specific interaction the individual neuromasts have with the local hydrodynamic field, and the relative intensity of stimulation achieved. The differences in shape, size, and location of hair cells will lead to different interactions with the local fluid flow, and potentially to differences in detection threshold sensitivity. What is needed first is a series of studies which provide a basis for determining detection threshold levels.

From the experiments of Coombs and Janssen (1990), a wall strain threshold can be determined for the Mottled Sculpin trunk lateral line superficial neuromast. They 


\section{Surface strain rate calculations and predictions due to osc. sphere at phase $=0$}

A

Strain rate at surface

$s^{-1}$

$+.80 \mathrm{e}-02$

$+1.70 \mathrm{e}-02$

$1.60 \mathrm{e}-02$
$+.50 \mathrm{e}-02$

$+.50 \mathrm{e}-02$

$.50 \mathrm{e}-02$
$+.40 \mathrm{e}-02$

$+.30 \mathrm{e}-02$

$+.200-02$

$1.10 \mathrm{e}-02$

$1.00 \mathrm{e}-02$
$9.00 \mathrm{e}-03$
$8.00 \mathrm{e}-03$

$9.00 \mathrm{e}-03$

$7.00 \mathrm{e}-03$

$6.000-03$

$5.00 \mathrm{e}-03$

3.00e-03

$2.00 \mathrm{e}-03 \quad \mathrm{Z}$

$2.00 e-03$
$1.000-03 x-1 x$

$0.00 e+00$
B Predicted strain rate from $1 \mathrm{~mm}$ away

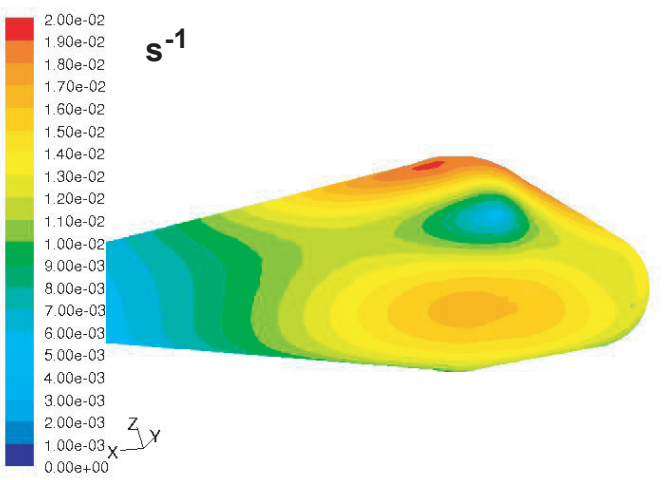

D Coarse grid predicted strain rate from $2 \mathrm{~mm}$ away

C

Predicted strain rate from $2 \mathrm{~mm}$ away

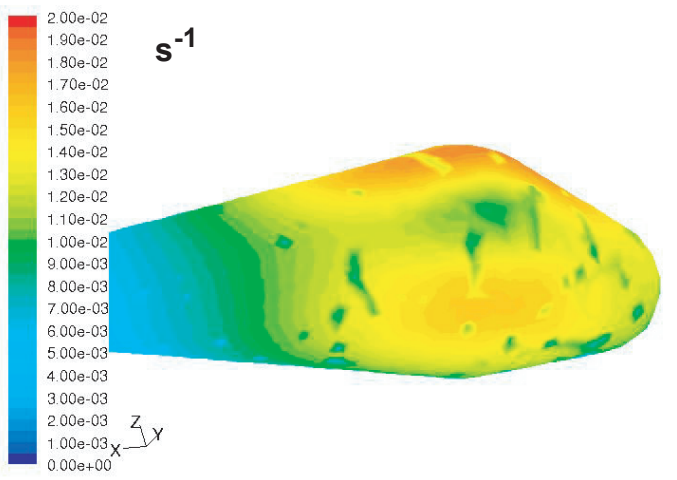

Figure 4-15: Validation of the strain rate predictions on the surface of a fish. Plots A-C use a resolved boundary layer mesh (see text), while plot D uses a coarse grid mesh (2 mm node spacing). (A) Numerical calculation of the strain rate at the fish surface. (B) Surface strain rate predicted using equation 4.7 , with $U_{\infty}$ equaling the velocity $1 \mathrm{~mm}$ away. (C) Same as B, but at $2 \mathrm{~mm}$ away. (D) Same as C. 
present velocity thresholds for the superficial neuromasts determined neurophsyiologically, as measured at the location of the fish (without the body present), due to a nearby $(15 \mathrm{~mm})$ oscillating sphere. They measured peak-to-peak amplitudes, which is equivalent to including the presence of a wall. From their figure 7 data, the velocity outside the boundary layer falls in a range of $9-32 \mu \mathrm{m} / \mathrm{s}$ at $10 \mathrm{~Hz}, 25-79 \mu \mathrm{m} / \mathrm{s}$ at 50 $\mathrm{Hz}$, up to a range of $28-158 \mu \mathrm{m} / \mathrm{s}$ at $100 \mathrm{~Hz}$. They didn't present the heights of the neuromasts, so neither a velocity threshold nor approximate strain rate threshold can be determined. However, knowing the frequencies used, a wall strain rate threshold range of $S_{\text {wall }}=0.07-0.25 \mathrm{~s}^{-1}$ at $10 \mathrm{~Hz}, S_{\text {wall }}=0.44-1.4 \mathrm{~s}^{-1}$ at $50 \mathrm{~Hz}$, up to a range of $S_{\text {wall }}=0.7-4.0 \mathrm{~s}^{-1}$ at $100 \mathrm{~Hz}$, can be determined using equation 7 .

Kroese et al. (1978) used a sphere of radius $\mathrm{a}=1.55 \mathrm{~mm}$, oscillating at $\mathrm{f}=20 \mathrm{~Hz}$ parallel to the fish surface, with a displacement amplitude of $\mathrm{A}=4 \mu \mathrm{m}$, and placed at a distance of $\mathrm{r}=3.75 \mathrm{~mm}$ from the surface. They assumed a hair cell height of $100 \mu \mathrm{m}$, which is fully immersed in the boundary layer flow. Figure 4-16 shows that the hair cell experiences a range of velocities from $0 \mu \mathrm{m} / \mathrm{s}$ at the base to about $3 / 4$ of their predicted value of $38 \mu \mathrm{m} / \mathrm{s}$ at the tip. The approximate strain rate threshold would be $S_{\text {approx }} \approx(30 \mu \mathrm{m} / \mathrm{s}) /(100 \mu \mathrm{m})=0.30 \mathrm{~s}^{-1}$. By using the wall strain rate threshold, neither the hair cell height nor the specific velocities along its surface are required. Using their parameters, and noting that $U_{0}=A \omega=502.7 \mu \mathrm{m} / \mathrm{s}, f(\alpha=0)=1$, and $C(a, \omega)=1.13$, the wall strain rate threshold would be $S_{\text {wall }}=0.45 \mathrm{~s}^{-1}$.

From Dinklo's (2005) data and from his superficial neuromast models (based on the work of van Netten, 2006), an approximate strain rate threshold can be determined for the zebrafish superficial neuromasts. The height of the neuromast, based on a fit of the model to the data, was $34 \mu \mathrm{m}$. For velocity thresholds of $12.3 \mu \mathrm{m} / \mathrm{s}$ at $0 \mathrm{~Hz}, 10 \mu \mathrm{m} / \mathrm{s}$ at $10 \mathrm{~Hz}$, and $27 \mu \mathrm{m} / \mathrm{s}$ at $100 \mathrm{~Hz}$, this would give $S_{\text {approx }} \approx$ $(12.3 \mu \mathrm{m} / \mathrm{s}) /(34 \mu \mathrm{m})=0.36 \mathrm{~s}^{-1}$ at $0 \mathrm{~Hz}, S_{\text {approx }} \approx 0.34 \mathrm{~s}^{-1}$ at $10 \mathrm{~Hz}$, and $S_{\text {approx }} \approx$ $0.79 \mathrm{~s}^{-1}$ at $100 \mathrm{~Hz}$. For the superficial neuromast, the best sensitivity is in a range of 0-10 Hz. There was not enough information to calculate the wall strain rate threshold.

With a range of strain rate detection thresholds now determined, we may reexamine the cases of the blind cavefish and that of the Mottled Sculpin, to see what 
threshold sensitivity levels are implied by the behavioral responses of the fish. For the behavioral experiments with the Mottled Sculpin (Coombs et al., 2001), the oscillating source strength and frequency are provided, enabling the calculation of wall strain rate thresholds. From the experimental set up, peak-peak velocity amplitudes of 0.11 $\mathrm{m} / \mathrm{s}$ or $0.18 \mathrm{~m} / \mathrm{s}$ were used for the $10 \mathrm{~Hz}$ and $50 \mathrm{~Hz}$ signals, respectively. Using these values for the $3 \mathrm{~mm}$ sphere at a distance of $6 \mathrm{~cm}$ away, with $\mathrm{C}=1.09$ for $10 \mathrm{~Hz}$ and 1.04 for $50 \mathrm{~Hz}$, equation 4.9 predicts $S_{\text {wall }}=0.06 \mathrm{~s}^{-1}$ for $10 \mathrm{~Hz}$, and $S_{\text {wall }}=0.21 \mathrm{~s}^{-1}$ for $50 \mathrm{~Hz}$. These values are slightly below the low values of the threshold ranges already presented. For the sphere a distance of $3 \mathrm{~cm}$ away, these same calculations predict $S_{\text {wall }}=0.48 \mathrm{~s}^{-1}$ for $10 \mathrm{~Hz}$, and $S_{\text {wall }}=1.66 \mathrm{~s}^{-1}$ for $50 \mathrm{~Hz}$, which are well above threshold.

In the case of the blind cavefish (Abdel-Latif, 1990), a range of displacement amplitudes for the oscillating sphere caused a positive behavioral response, at least at $50 \mathrm{~Hz}$ and $75 \mathrm{~Hz}$. Using equation 4.9, that gives a strain rate threshold range of $S_{\text {wall }}=0.0023-0.016 \mathrm{~s}^{-1}$ at $50 \mathrm{~Hz}$, and $S_{\text {wall }}=0.0038-0.026 \mathrm{~s}^{-1}$ at $75 \mathrm{~Hz}$. If the blind cavefish has a superficial neuromast height of $200 \mu \mathrm{m}$ (Teyke, 1988), then the velocity threshold would range from $0.14-0.96 \mu \mathrm{m} / \mathrm{s}$ at $50 \mathrm{~Hz}$ to a maximum of $0.19-1.3 \mu \mathrm{m} / \mathrm{s}$ at $75 \mathrm{~Hz}$, and the approximate strain rate would be $S_{\text {approx }} \approx(0.7-5) \cdot 10^{-3} \mathrm{~s}^{-1}$ at

$50 \mathrm{~Hz}$, up to $S_{\text {approx }} \approx(0.9-7) \cdot 10^{-3} \mathrm{~s}^{-1}$ at $75 \mathrm{~Hz}$. These are extremely sensitive thresholds, even acknowledging the possibility of signal startup transients, which from numerical simulation briefly increased the wall strain rate up to 4 times it steady value.

\subsection{Discussion}

One goal of this paper was to devise a simple method of obtaining information about the threshold detection abilities of superficial neuromasts. The method is meant to be broad enough to allow for cross-experiment comparisons, even when data is incomplete or missing. To some extent, this is made possible when more information is known about the boundary layer velocity profile. From this information, velocities at the tip of the superficial neuromast can be obtained. 
There needs to be some thought as to how detection thresholds are to be defined. The main reason is that most neuromasts are partially or fully immersed within a boundary layer flow that is frequency dependent. To date, only the work of van Netten (2006) has attempted a detection threshold definition that includes the effects of the boundary layer, and that definition is for canal neuromasts detecting fluid acceleration at the body surface. Dinklo (2005) and Humphrey et al. (1993) have included the effects of boundary layer flow for superficial neuromasts and hair cells, respectively, but neither defined detection thresholds as their purposes were of a different nature.

Using the approximate strain rate threshold definition, cross species results can be compared. Differences in the height of the superficial neuromasts factor in to the detection threshold measurements, providing a more meaningful comparison. When only the oscillation frequency is known (i.e. the velocity and the neuromast height are unknown), the surface strain rate threshold definition can be utilized for cross species detection threshold comparisions. This can be very useful in the case of behavioral experiments, where information is often known about the stimulus source, but not about the hydrodynamic flow within the surface boundary layer and at the location of lateral line organs.

One caveat to this method is that its use relies on the assumption that the velocity profile within the boundary layer can be described using Stokes (1851) solution for an oscillating plate (or fluid). This is an assumption that needs to be validated under a

wider range of conditions, but at least numerically it is valid when the surface can be approximated as a wall. And it is validated numerically for the case of an oscillating sphere, which is used quite extensively in lateral line experiments.

\subsubsection{Quantitative determination of canal vs. superficial lat- eral line sensitivity}

Returning to the original dilemma of whether the superficial or canal lateral line is used for prey detection purposes (i.e. an oscillating sphere stimulus), the use of these threshold definitions can help to resolve it. From the data presented in 
three neurophysiological experiments, either the velocity threshold and approximate strain rate threshold (Dinklo, 2005), or the wall strain rate threshold (Coombs and Janssen, 1990), or both (Kroese et al., 1978), could be extracted and used as limited benchmarks for further comparisons.

The Blind Mexican Cave Fish of the Abdel-Latif et al. (1990) experiment showed behavioral results that would indicate extremely sensitive superficial neuromast detection thresholds. From the oscillation strength and distance of the sphere presented, the velocities at the surface of the fish, and at the tips of the cupulae, are at least an order of magnitude smaller that what seems to be required for detection. In addition, the strain rate threshold metrics are in some cases two orders of magnitude smaller than what seems to be required. Although there can be strong transient surges in signal strength in the start up of the source stimulus (Kroese et al., 1978), it is unlikely that they are strong enough to account for this difference (numerical simulation showed gains of 4 times).

These quantitative results seem to indicate that there is some other mechanism at work helping the Blind Mexican Cave Fish to detect the oscillating stimulus. Coombs et al. (2001) suggest that the inner ear of the fish might aid in the process of detection. By the way they were conditioned to respond, they may have only needed to be alerted to the presence of the stimulus, and then they could find it without spatial information.

For the Mottled Sculpin of the Coombs et al. (2001) experiment, the wall strain rate thresholds calculated at the furthest source distance described fall just below acceptable ranges for the $10 \mathrm{~Hz}$ and $50 \mathrm{~Hz}$ signals. At the closest location of the oscillating sphere, the wall strain rates far exceed threshold. It appears that the superficial neuromasts were adequately stimulated, and yet not utilized for prey detection, according to the experiment. This raises the question of why? To answer this question, it will be useful to compare relative levels of stimulation between the canal and superficial lateral line organs, to see if there are significant differences. If so, one hypothesis is that the fish learn to use their primary detection mechanism, their canal lateral line system, to detect and localize the prey. When that is removed, 
the signals to their superficial lateral line system may be too weak in comparison, and so ignore them.

Figures 4-12 and 4-13 show the oscillating sphere located to the side of the trunk lateral line. This is very similar to the Coombs et al. (2001) experiment, with the sphere located $3 \mathrm{~cm}$ and $6 \mathrm{~cm}$ away from the longitudinal midline of the sculpin fish. The maximum (normalized) stimulation for the superficial neuromasts is roughly $2 \cdot 10^{-3}$ when the sphere is $3 \mathrm{~cm}$, and $2 \cdot 10^{-4}$ when the sphere is $6 \mathrm{~cm}$ away. That corresponds to surface strain rate values of $0.48 \mathrm{~s}^{-1}$ and $0.05 \mathrm{~s}^{-1}$ at $10 \mathrm{~Hz}$, and 1.66 $\mathrm{s}^{-1}$ and $0.17 \mathrm{~s}^{-1}$ at $50 \mathrm{~Hz}$. The maximum (normalized) stimulation for the canal neuromasts is roughly $1.5 \cdot 10^{-3}$ when the sphere is $3 \mathrm{~cm}$ away, and $1.5 \cdot 10^{-4}$ when its $6 \mathrm{~cm}$ away. Using equation 4.10, that corresponds to accelerations of $30 \mathrm{~mm} / \mathrm{s}^{2}$ and $3 \mathrm{~mm} / \mathrm{s}^{2}$ at $10 \mathrm{~Hz}$, and $522 \mathrm{~mm} / \mathrm{s}^{2}$ and $52 \mathrm{~mm} / \mathrm{s}^{2}$ at $50 \mathrm{~Hz}$.

For the superficial neuromasts, minimum strain rate thresholds for the sculpin are approximately $0.07 \mathrm{~s}^{-1}$ at $10 \mathrm{~Hz}$ and $0.44 \mathrm{~s}^{-1}$ at $50 \mathrm{~Hz}$. That means that when the sphere was $3 \mathrm{~cm}$ away from the fish, the relative stimulation was approximately 7 times threshold at $10 \mathrm{~Hz}$, and approximately 4 times threshold at $50 \mathrm{~Hz}$. However at $6 \mathrm{~cm}$, it was below threshold, as already determined.

For the canal neuromasts, van Netten (2006) determined that detection threshold range is approximately $0.1-1 \mathrm{~mm} / \mathrm{s}^{2}$. Even using the less sensitive value, the canal neuromasts were stimulated 30 times threshold at $10 \mathrm{~Hz}$, and 522 times threshold at $50 \mathrm{~Hz}$ when the sphere was $3 \mathrm{~cm}$ away. When the sphere was $6 \mathrm{~cm}$ away, the canal neuromast stimulation was only 3 times threshold at $10 \mathrm{~Hz}$, and 52 times threshold at $50 \mathrm{~Hz}$.

These are large differences in stimulation relative to the canal and superficial lateral line system, and it strongly supports the hypothesis that the canal lateral line is the more sensitive organ when it comes to detecting vibrating signals. Secondly, since the acceleration detection threshold stays fairly constant over the whole range of frequencies, an increase in source frequency without change source amplitude causes huge increases in the received signal amplitude. This does not appear to be the case for the superficial neuromasts, whose relative levels of stimulation stay fairly constant 
over larger ranges of frequencies. This again highlights the need to better understand superficial neuromast stimulation within the boundary layer.

\subsubsection{The effect of body parts on the dipole signal to the lateral line system}

Another goal of this chapter was to examine how the hydrodynamic field of an oscillating sphere becomes altered around fish bodies with shapes more realistic than the typical axisymmetric approximations. In particular, the effect that extended pectoral fins has on the received dipole signal on the lateral line was investigated. From the computational fluid dynamics simulations, it appears that the extended fins have relatively little effect on the pressure gradient distribution over the body surface, and only a small localized effect on the flow over the body surface. The reason for this is that in this still water case, and for an oscillating sphere with its displacement amplitude much smaller than its radius, the flow field remains potential in nature. Viscous and non-linear effects that might have been significant near the body and around fins are in fact negligible. This also explains why the virtual body predictions so closely match the numerical simulation results.

The fact that the lateral line system of the Mottled Sculpin goes around the pectoral fins is likely of morphological significance, since it appears to avoid any distortion in the received signal. In addition, the lateral line has locations and orientations that specifically sample the pressure and velocity fields in a unique ways. For example, when the prey is swimming vertically past the fish, in the dorsal/ventral plane, the pressure gradient would be flat around the trunk lateral line, whereas the infraorbital lateral line locations would more readily detect these motions. Hassan (1993) has pointed that the combinations of orientations of the lateral line sections theoretically allow for unique encoding of any orientation and location of the dipole source.

Figure 4-8 showed that if the fish could integrate the signal among the different sections of its full lateral line system (i.e. trunk, mandibular, etc.), the length of the lateral line would in effect be doubled. This would allow the fish to localize objects 
at farther distances. Just using the distance between zero crossings as an example, it could accurately localize a dipole source 1.4 BL (i.e. $2 / \sqrt{2}$ ) away.

There is some evidence against this hypothesis, which suggests that information processing by the fish is unilateral (Coombs et al., 2000). The approach strategies of the Mottled Sculpin show that it approaches in steps, orienting one side at a time. In experiments where fish had one side denervated, there was no effect in its ability to detect and attack prey on its good side. Poor frontal performance suggests that spatial information is not integrated from side to side (Coombs et al., 2000). However, the numerical simulations at least show that the integrity of the signal is upheld in using the full lateral line system.

\subsubsection{Canal and superficial lateral line use in prey detection and localization}

Based on the previous analysis of sphere source strength and thresholds of detection, the results showed that the canal neuromasts are more sensitive to prey detection than the superficial neuromasts over a larger range of frequencies. This should prove quite valuable for a fish on the hunt, since a moving fish will have its superficial neuromasts saturated by the constant flow over its surface, while the canal neuromasts should remain sensitive to velocity perturbations (accelerations) in the water.

When the source is strong enough for the superficial neuromasts to be activated, a great deal more information is available to the fish. Superficial neuromasts have the potential to respond to the full directionality of the local hydrodynamic flow, bending in directions determined by the flow. As a simple demonstration, figure 411 shows that superficial neuromasts placed at locations similar to the canal lateral line give localization information in a similar manor as the canal lateral line system

does. Having superficial neuromasts polarized in orthogonal directions allows two independent methods of source localization.

In cases where the superficial neuromasts cover more of the body surface, more flow information is available for dipole localization. Hassan (1993) showed that complete 
excitation vectors (averaged angle and magnitude) on either side of the body, and the front sides, uniquely encode the location and orientation of the source dipole. Working together, the information from canal and superficial lateral line neuromasts can provide a more accurate rendering of the actual hydrodynamic vector field. Canals are permanently oriented on the fish, and therefore respond only to the pressure gradient components aligned with the canals. As the flow moves increasingly to the perpendicular of the canal alignment, stimulation for the canal neuromasts and superficial neuromasts in alignment with the canal will die down while stimulation to superficial neuromasts aligned to the perpendicular will increase (Hassan, 1993).

This chapter focused on spatial signals to the lateral line system, though it is likely that temporal information will be as useful for the fish in detection and localization of prey. For example, a passing dipole source should induce a signal whose peaks and zeros move along the lateral line in accord with the prey's changing location. The change in location of peaks and zeros encode velocity vector information. As more realistic signals are considered, the combination of spatial and temporal information might enable the fish to identify and localize the prey. This should be an area of future investigation. 


\section{Velocity boundary layer profile at center point on wall due to a $20 \mathrm{~Hz}$ oscillating sphere}

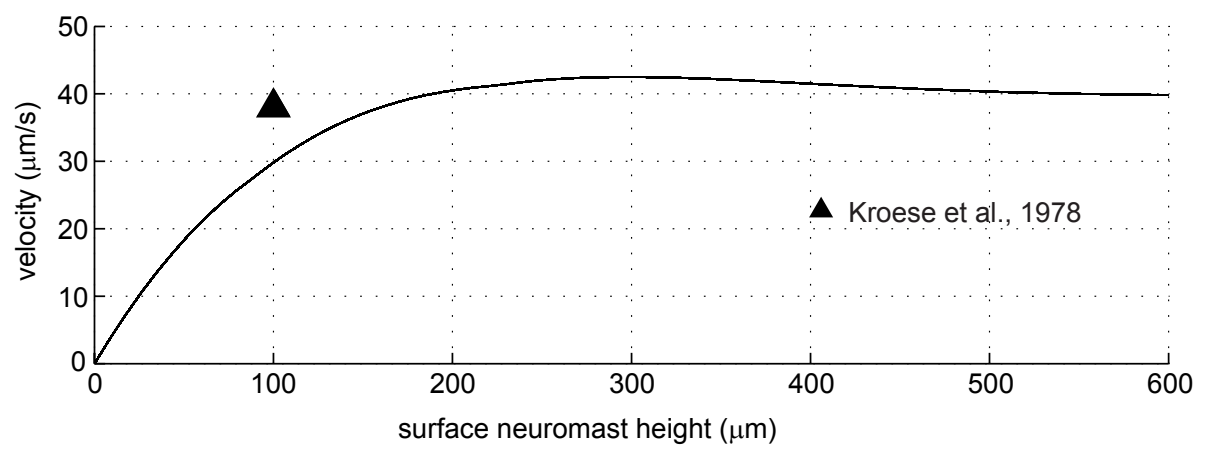

Figure 4-16: The boundary layer flow (solid line) due to an oscillating sphere next to fish surface is calculated using equation 4.8. Potential flow theory (such as used by Kroese et al. (1978) does not account for reduced velocities that affect the superficial neuromast when it lies within the boundary layer. 


\section{Chapter 5}

\section{Thresholds of detection and saturation in moving water}

\subsection{Introduction}

The core question of this thesis is how fish are able to detect small amplitude signals against large amplitude noise signals. In terms of signal detection theory, the signal must distinguish itself from the noise. If it is a monotone signal, then the signal-tonoise ratio (SNR) at that frequency must be larger than surrounding frequencies. To what extent it must be larger is unknown a priori, and can only be determined by matching hydrodynamic conditions and signals to behavioral and neural responses. Secondly, the encoding process of the hydrodynamic information involves mechanical filtering (boundary layer and canal structure), mechanical motions of the neuromast, hair cell transduction into neural impulses, and modulation of those signals by feedback from the brain. The previous chapters of this thesis form the foundation with which to address this question.

The input-output mechanical motions of the superficial and canal neuromasts have been modeled for the Mottled Sculpin. The lateral line response to an oscillating dipole stimulus has been quantified in still water conditions, with evidence that the canal lateral line will be the more sensitive detector. Finally, the study of Kanter and Coombs (2003) provides the behavioral response of the Mottled Sculpin to an 
oscillating dipole source in a background flow. They quantify the detection threshold using the peak-peak velocity of the oscillating sphere at the moment of fish response.

Coombs et al. (2007) further quantify the basic conditions of the background flow in the Kanter and Coombs (2003) experiment. Average velocity and vorticity flow conditions around the sculpin show a region of decreased flow, and they conjecture that it might lead to increased SNR for the lateral line in the immediate vicinity. Secondly, there are regions of recirculation by the pectoral fins, and vortical shedding. What remains unknown from both studies is the level of turbulence at the time of signal detection in the first experiment.

In this chapter, the Kanter and Coombs (2003) experiment is simulated numerically. The 3D fish body is approximated, and the locations of the lateral line are placed on the body to match locations of the real fish. A sphere of the same size (6 mm diameter), same location ( $5 \mathrm{~cm}$ away from the side of the fish at the point of pectoral fin insertion), and same oscillation frequency $(50 \mathrm{~Hz})$, is used in this simulation. Since the levels of turbulence are unknown, a number of simulations with varying levels of turbulence are used to try and bound the results of Kanter and Coombs.

The difficulty lies in trying to 'decide' what constitutes detection in these simulations. In the Kanter and Coombs experiment, the fish would respond at the appropriate stimulus level. In this simulation, threshold of detection must be defined in a way that is realistic and plausible. The previous chapters were able to do this for still water conditions, but evidence suggests that detection mechanisms change when in moving water conditions.

\subsubsection{In moving water, saturation for superficial, but not for canal neuromasts}

Studies showed that there were two main types of neural responses when fish are placed in background flow environments. Type I fibers responded to an increase in the flow rate, and often reached saturation with increasing flow speed. Type II fibers remain unchanged in their basic spontaneous neural activity when background flow 
was added. The speculation is that type I fibers lead to superficial neuromasts, while type II fibers lead to canal neuromasts. Not all fibers fall into the type I or type II category - collectively, neuromasts exhibit a range of responses to the flow. Some type II fibers could be mis-categorized as type I fibers due to damming pressure which builds up in front of the fish, and therefore showed a response to flow increase (Engelmann et al., 2003). Secondly, all fibers show responses when the flow speed is increased high enough (Chagnaud et al., 2008).

The explanation given as to why type I fibers show a response to background flow and type II fibers do not is largely based on the locations of the neuromasts being enervated. Superficial neuromasts reside on the surface of the fish, and although they lie within a protective boundary layer, they still experience a strong shear force as the flow moves past. This forces the neuromast to bend with the flow. Changes in the flow due to movements by the fish, or from shedding and recirculation from body generated self-flow, cause the response of the type I fibers to be non-uniform. In addition, Engelmann et al. (2003) postulate that the observed burst-like responses for flow sensitive fibers to unidirectional flow were likely caused by local pockets of turbulence around the trunk lateral line.

Type II fibers are part of the canal neuromast system, which respond to the pressure gradients at the surface of the fish. If the flow is fairly uniform, flat areas on the fish should produce very low pressure gradients. In areas of body curvature, a larger pressure gradient will exist, causing a deflection of the canal neuromasts in a manor similar to the superficial neuromasts. This is especially likely near the front of the fish (Engelmann et al., 2003).

\subsubsection{Background flow and signal masking}

There is evidence that background flow decreases the fish's signal detection ability for type I fibers, but not for type II fibers. One effect is called signal masking, where there is decrease in the evoked spike rate to a signal in flowing water, as compared to the evoked spike rate to that same signal in still water. Engelmann et al. (2002) found that flow sensitive fibers (type I) to the posterior lateral line showed masking to 
an oscillating dipole signal with increase of flow rate, whereas flow insensitive fibers (type II) did not show signal masking. Chaugnaud et al. (2006) found similar results to Englemann et al. (2002) for fibers enervating the anterior lateral line. Kanter and Coombs (2003) and Bassett et al. (2006) found that fish can detect oscillating signals which are orders of magnitude smaller than surrounding flow, and in a previous work, Coombs and Janssen (1990) were able to link behavioral responses to signal detection to canal neuromast (type II) stimulation.

Reduced detectability for superficial neuromasts might be expected in flowing water, since their exposure to the flow is only tempered by the surface boundary layer they reside in. Voight et al. (2000) found that flow sensitive fibers had a non-adapting, linear response to flow increase. Overall, the spike rate increased in a linear fashion with increasing flow rates. An increase in mean spike rate is likely due to an increase in the root mean square deflection of the neuromast responding to a.c. flow fluctuations. An additional stimulus, such as an oscillating dipole, should be superposed onto the flow signal. If the stimulus signal is small compared to the flow signal, then the additional increase in hair deflection may be too small to detect well.

There is evidence that hair cells can adapt to repetitive patterns and mean flow signals by reducing the spike rate (Eatock, 2000). This could explain the decrease in the evoked spike rate of an oscillating dipole signal as compared to the same signal in still water conditions (Engelmann et al., 2002; Chaugnaud et al., 2006). The hair cell would likely adapt to the flowing water by trying to decrease the overall signal, which includes both the background flow signal and the stimulus signal.

In the studies of Englemann et al (2002) and Chaugnaud et al. (2006), the type II fibers exhibited only minor signal masking of an oscillating dipole signal in background flow. This is consistent with the fact that the mean spike rate did not increase, or increased only a minor amount relative to its mean spike rate in still water conditions. However, there is some evidence for adaptation, for some fibers showed a decrease signal masking, which can only result from a lowering of the mean spike rate overall.

In contrast to the neurophysiological studies, some behavioral studies show ev- 
idence of signal masking for canal neuromasts. Kanter and Coombs (2003) found through behavioral experiments that there is approximately a threefold increase in the threshold of detection for an oscillating dipole signal from $0 \mathrm{~cm} / \mathrm{s}$ to $2 \mathrm{~cm} / \mathrm{s}$ flow, and then a twofold increase in threshold of detection from $2 \mathrm{~cm} / \mathrm{s}$ to $8 \mathrm{~cm} / \mathrm{s}$ flow. If the hair cells can adapt to low frequency signals, why should there be any increase in the mean spike when there is flowing water? Evidence suggests that unsteady, a.c. flow fluctuations prevent the hair cell from adapting (Chaugnaud et al., 2006). Perhaps the presence of turbulence, which increases the amplitude of a.c. flow fluctuations over a range of frequencies, could be factor in explaining differences in detection ability between the experiments.

\subsubsection{Biological basis for signal encoding, adaptation, and filtering}

The fish can use a variety of methods to encode, adapt to, and filter the synaptic signals sent by the afferent nerves to the brain (Popper and Fay, 1999). Encoding can be in the form of synchronizing the spontaneous nerve activity of the hair cell to the envelope of an amplitude modulated signal, increasing the mean spike rate in response to increases of stimulus amplitude and stimulus frequency, or phase-locking the nerve responses to the signal frequency. Adaptation can be in the form of reducing the mean spike rate, or canceling out a repetitive signal. Filtering can occur through frequency selection (phase-locking), or through preferential sensitivity to specific frequencies. For example, some neuromasts resonate at particular frequencies based on their size and stiffness. The neuromast motion will have larger deflections than for signals of similar amplitude at other frequencies.

Each hair cell has a particular level of spontaneous activity, falling into categories of having random interspike-intervals, having a bimodal distribution of interspikeintervals, having highly regular activity, or having none at all (Popper and Fay, 1999). Those with higher spontanteous rates tend to synchronize spikes to the phase of the stimulus signal, whereas those with low or no spontanteous activity only activate once 
threshold is reached along the stimulus waveform. As the amplitude of the stimulus wave increases, spikes are triggered at earlier points along the waveform, until at some point spikes are triggered at all points along the waveform. Therefore, these afferents encode excitation level in terms of a time delay.

Furukawa et al. (1986) provides evidence that the hair cell synapse of the saccular afferents of goldfish functions as a multiple-release-site model. Summarizing the key results of Furukawa et al. and other researchers, Popper and Fay (1999) explain that the hair cell has many presynaptic release sites, each of which have a different threshold which is only activated if the stimulus reaches threshold. There is a single synaptic vesicle at each release site, which remains empty after a release until refilled from a larger reserve. In addition, vacant sites are replenished in order of high threshold to low threshold, and the low threshold sites remain vacant as long as the stimulus is above its threshold. The main effects of the multiple-release-site model for the hair cell are that the excitatory postsynaptic potentials reach multiple levels in amplitude, and lessen over the duration of a stimulus tone.

One form of adaptation by the hair cells is single-tone and two-tone rate suppression. Some hair cells will suppress the firing of synapses when frequencies pass a critical level, often call the characteristic frequency (Pooper and Fay, 1999). Twotone rate suppression occurs when a second tone at a higher or lower frequency is introduced, and the hair cell lessons the evoked spike rate of the first tone (which is near threshold level to begin with). Furthermore, signals generated by the fish's own motions, such as breathing, can be canceled out by the brain (Bodznick et al., 2003).

Eatock (2000) describes another type of hair cell adaptation to sustained deflections. The tiny links which connect the stereocilia together shift their locations along the stereocilia in order to relieve stress. In other hair cells, $\mathrm{Ca} 2+$ binds directly to the transduction channels. In both cases, the result is to lessen the transduction current at low frequencies. This means that that canal and superficial neuromasts have the ability to adapt to d.c. and low frequency a.c. stimulus signals. This is a type of filtering which may prove very useful to the fish while swimming or holding station in background flows. 
In summary, fish employ a number of different strategies to both encode and filter stimulus signals to the hair cell. When the stimulus is of large amplitude, the hair cell can lower the spike rate generated by the large deflection by adjusting the location of stereocilia links, by binding transducer channels with $\mathrm{Ca} 2+$, or by raising the hair cell threshold (i.e. leaving low threshold sites vacant). They have the ability to lower the synaptic firing rate for d.c. and low frequency a.c. signals, and suppress signals generated from their own motions or breathing. They have the ability to phase lock to particular frequencies (spectral filtering), or to follow amplitude modulated signals (temporal filtering). The mean spike can encode both amplitude and frequency of the stimulus signals.

\subsubsection{Towards a modified definition of threshold of detection and saturation}

In still water, the threshold of detection and saturation was previously defined using neuromast displacement. In particular, van Netten's (2006) theoretical basis for detection threshold was applied to the Mottled Sculpin by matching the threshold tuning curves of Coombs and Janssen (1990). However, the displacement of the neuromast is only part of the encoding process. The bending of the hair cell signals the cell to release neurotransmitters. The rate of synaptic signaling is part of a complex process involving the hair cell, the type of signal, and a feed-forward / feedback process with the brain.

The nonlinearity of the stimulus encoding process by the hair cell has made it difficult to characterize. Most experiments use a combination of phase-locking and mean spike rate to generate frequency threshold tuning curves (Coombs and Janssen, 1990; Engelmann et al., 2002; Chaugnaud et al. 2006). Other experiments have used reverse correlation (Fay, 1997), where the hair cell responds to a white (flat, broadband) spectrum. From the temporal perspective, this is the hair cell response to an impulse signal (Curcic-Blake and van Netten, 2005). In another characterization, the sound pressure level is held constant while the frequency is varied, and the mean 
spike rate is measured (Fay, 1990). Each of these methods can produce different tuning curves, and sometimes with apparently contradictory results (Popper and Fay, 1999).

However, at stimulus amplitudes near the detection threshold level, the hair cell behaves in a linear fashion (Kroese et al., 1978). Therefore, a detection threshold definition based on mean spike rate, phase-locking, or even neuromast displacement should be in agreement. Coombs and Janssen (1990) were able to define signal detection threshold in way which resulted in agreement between neurophysiological and behavioral responses for the Mottled Sculpin in still water conditions. In one method, the detection threshold was defined as the stimulus level at which the evoked spike rate rose above the mean spontaneous firing rate. In another method, the threshold was defined as the stimulus level at which the phase-locking synchronization coefficient reached 0.4. Finally, both these neurophysiological thresholds matched a behavioral response threshold, defined as the stimulus level that the fish made a turn towards the oscillating source.

The frequency threshold tuning curves of Coombs and Janssen (1990), in combination with the canal and superficial neuromast motion models, allow for the prediction of behavioral responses based on the type of stimulus input in still water conditions. In situations where the fish is swimming, or holding station against moving background flows, the hair cell responses can behave in a nonlinear fashion, and the definitions for detection and saturation threshold become more difficult.

Perhaps the safest way to proceed is to use detection threshold definitions that reflect the biology of the encoding and filtering process. The neuromast models developed for the still water response can be used to show the physical motions of the neuromast to the hydrodynamic signals present. Though neuromast deflections might otherwise indicate saturation, it will be assumed that the neural responses of the hair cells will find a way to adapt to the mean signal, automatically recalibrating the detection and saturation threshold levels. Secondly, it will be assumed that the hair cells cannot adapt to broadband a.c. flow fluctuations (Chaugnaud et al., 2006). The root mean square of the flow fluctuations will be the baseline noise with which the stimulus signal must compete to be detected. Finally, phase-locking has the ability 
to isolate particular frequencies. Therefore, when the spectral peak of the stimulus signal is significantly larger than surrounding peaks, detection of the signal should occur.

\subsection{Methods}

\subsubsection{Numerical set up}

The domain of the numerical set up was given in the previous chapter. The shape of the fish was created to approximately match that of the Mottled Sculpin, and the lateral line locations placed spatially on the fish to match the locations of the real fish. The sphere is $6 \mathrm{~mm}$ in diameter, oscillates at $50 \mathrm{~Hz}$ parallel to the long axis of the fish, and is placed $5 \mathrm{~cm}$ away from the point of pectoral fin insertion on the side of the fish ( $5.5 \mathrm{~cm}$ from the longitudinal midline of the fish), and is located at about the same height as the trunk lateral line.

Large eddy simulations were performed in order to capture the time-dependent flow conditions. This method essentially models the energy of the flow within each grid cell, which is the smallest spatial scale resolution possible, and allows it to interact with larger spatial scales of the flow which are directly simulated. This allows for a coarse grid meshing, while accounting for highly resolved flow interactions.

Further justification for a coarse grid mesh, which uses a node separation of $2 \mathrm{~mm}$ around the body, is that the canal lateral line has pore openings of that approximate separation. The spatial averaging of the real fish is then matched in these numerical simulations. Additionally, it enables reasonable computational requirements on memory, whereas a fully resolved 3D grid mesh is well beyond any resources available.

Levels of turbulence were simulated using a spectral method (Smirnov et al., 2001) at the flow inlet, and allowed to propagate downstream. Basically, fluctuations are added to the mean flow vectors, whose spatial and temporal statistics match predetermined turbulence spectrums. By allowing the flow to evolve downstream, the flow can develop naturally occurring anisotropic structures. Turbulence is actually 
measured near the fish, so regardless of initial conditions, the results presented here will have a basis for broader comparison.

Even when there is no turbulence specified at the inlet, there is still turbulence that develops along the floor of the channel. Since the sculpin is placed on the floor, and is only $2 \mathrm{~cm}$ in height, it will feel some of the effects of the floor turbulence, which is present in the Large Eddy Simulation (LES) modeling. Turbulence will be measured as the root mean square of the velocity fluctuations, and reported in units of velocity rather than in percent turbulence intensity. This follows the standard convention seen in the experiments under study (e.g. Chagnaud et al., 2008). Secondly, it is the absolute levels of velocity fluctuation, and not the percent intensity, which is the stimulus to the lateral line.

\subsubsection{Thresholds of detection and saturation for the Mottled Sculpin in moving water enviroments}

To make sense of the experimental observations, it will be helpful to suggest a number of definitions for the threshold of detection and saturation. There will be two elements to consider. The first element is the physical response of the neuromasts to hydrodynamic stimuli. The flat-plate, spherical, and cylindrical models for the canal neuromast (see figure 3-15 and subsequent modifications), and the cylindrical model for the superficial neuromast (see figure 3-14), quantify the physical motions of the neuromasts in response to water motions adjacent the body of the fish. For example, a strong, steady shear force to either neuromast should result in a large, steady deflection of the neuromast and cilia inside. The second element is the hair cell adaptation to the neuromast deflections. The mean spike rate, and phase-locking properties do not necessarily match the neuromast deflection. Therefore, defining detection and saturation thresholds in moving water environments does not depend solely on the neuromast motions.

The first definition to consider is that of the base noise level. This is defined as the random noise inherent to the neuromast motions, caused by Brownian motion of the 
surrounding fluid, and caused by noise in the hair-cell gating apparatus (Dinklo, 2005; van Netten, 2006). Equivalently, each neuromast has its own level of spontaneous neural activity. In still water conditions, this will be called base-noise still $_{\text {. }}$

In moving water conditions, most hair cells will experience some form of adapta-

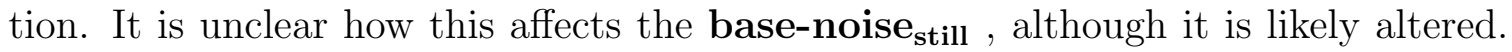
Therefore, in moving water, it will be called base-noise $\mathbf{m}_{\text {moving }}$.

The second definition is that a.c. fluctuations of flow are equivalent to noise. This definition is supported by Chaugnaud et al. (2006), who postulate that if the amplitude of the flow fluctuations exceeds the amplitude of the dipole signal fluctuations, then masking of the signal results. The root mean square of the a.c. fluctuations will be called flow-noise a.c.

Finally, detection threshold will be defined as the point that the root mean square of the desired signal (in this case the oscillating dipole signal) is greater than a defined noise level, either base-noise still , base-noise moving $_{\text {, flow-noise }}$ a.c. , or some combination. This leads to three hypotheses about the detection threshold level based on mean spike rate.

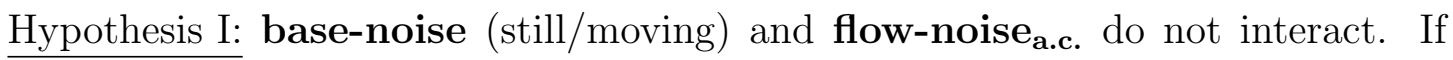
flow-noise a.c. $<$ base-noise, then threshold $=$ base-noise. If flow-noise a.c. $>$ base-noise., then threshold $=$ flow-noise a.c.

Hypothesis II: base-noise (still/moving) and flow-noise a.c. combine in a linear fashion. Threshold $=$ base-noise + flow-noise a.c.

Hypothesis III: base-noise (still/moving) and flow-noise a.c. combine in a nonlinear fashion. Threshold $=\mathrm{f}$ (base-noise, flow-noise $\left.\mathbf{a . c .}_{\text {. }}\right)$.

Determining which hypothesis is correct is partly the work of this thesis, but it depends on the internal mechanisms of the hair cell and needs biological verification.

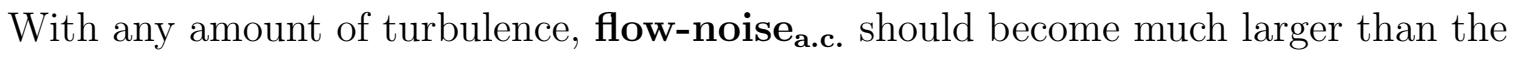
base-noise (still/moving). Hypothesis I, based on its ability to be calculated from 
hydrodynamic information from the simulations of this thesis and from experimental data available, is what is considered in this thesis.

The previous definitions and hypotheses were based on mean spike rate leading to signal detection. However, all studies mentioned also consider synchronization, or phase-locking by the hair cells. This will be most evident in the spectral domain.

Hypothesis IV: The spectral peak of the desired signal must be greater than the lower frequency spectral peaks.

Hypothesis V: The spectral peak of the desired signal must be greater than the spectral peaks of frequencies within proximate range of itself.

Each of these definitions must be applied to each neuromast, since they each reside at a different location. Hydrodynamic signals to the head of the fish are different than signals at the trunk or tail, and so at one instant of time there may be some neuromasts which are saturated (complete masking), some which are able to detect signals (partial masking), and others which cannot detect the signal at all (below threshold).

Saturation threshold depends on hair cell mechanics. It is not the absolute position of the cilia that determines the hair cell response, but the relative positions of the stereocilia and the kinocilium which causes a tensioning of the gating apparatus (Howard and Hudspeth, 1987). There are additional mechanisms which are not mechanical, but are related to hair cell chemistry, such as $\mathrm{Ca}+$ regulation (Eatock et al., 1987; Eatock, 2000). Saturation threshold is therefore defined in relation to a hair cell equilibrium point, which is constantly changing and adapting to the cilia motions. Tinevez et al. (2007) developed analytical models which can accurately duplicate many experimental observations. Nam et al. (2007) create a 3D virtual hair, containing individual stereocilia attached to a large kinocilium by spring gates. Their model keeps track of the states of the individual gates, and collectively responds in ways seen in experiments.

These models still indicate that the hair cell is not able to adapt well to a.c. fluctuations. Therefore the definition of saturation threshold in moving water, based 
on a simple approximation, is when the a.c. neuromast motions exceed a value of about $100 \mathrm{~nm}$ (Kroese and van Netten, 1989). That will be dependent upon the high pass filtering nature of the individual hair cells.

For this chapter, three situations are presented. First, the time dependent displacement of the neuromast is presented, in response to the pressure gradient signals upon it as determined by the flow field conditions. Second, the hair cell filtered signal, and the displacement of the neuromast which represents this signal, is considered. The characteristics of this high pass filter can be determined more generally from the experimental work of Eatock et al. (1987), or more specifically from the analytical or numerical models developed. Lastly, a high frequency $(>10 \mathrm{~Hz})$ filtering of the pressure gradient signal will be performed, so that the high frequency motions of the neuromast can be more easily visualized. This is not meant to represent biological reality, since fish are unlikely to have the mechanical capability to achieve this. However, is does represent reality in that these are the fluctuating motions superposed on the low frequency signals.

\subsection{Results}

\subsubsection{Determination of the base-noise level}

Since the base-noise moving is also not easily determined, for this thesis it will be

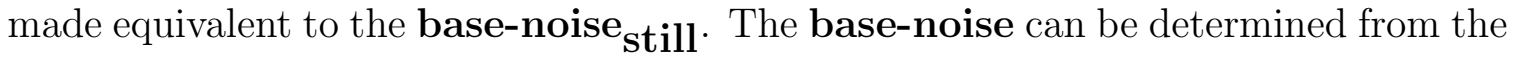
threshold of detection in still water conditions, as given in the Kanter and Coombs (2003) experiment. In still water conditions, the sphere with $50 \mathrm{~Hz}$ oscillations needed a velocity amplitude of approximately $1.2 \mathrm{~mm} / \mathrm{s}$ in order to elicit a response from the sculpin. Using the methods developed in 3D still water chapter, this condition is simulated in order to look at the stimulation at all lateral line locations. Then the points which give the maximum root mean square fluctuation will be equal to the base-noise level.

Figure 5-1 presents the results of this still water simulation. The maximum root 
mean square (rms) pressure gradient signal $(\mathrm{dp} / \mathrm{ds})$ is about $0.08 \mathrm{~Pa} / \mathrm{m}$, and is located at the point on the lateral line which is closest to the oscillating sphere. There next closest neuromast has a $\mathrm{rms} \mathrm{dp} / \mathrm{ds}$ of about $0.075 \mathrm{~Pa} / \mathrm{m}$, and there is also one point on the mandibular lateral line (not shown) which has a rms dp/ds that falls inbetween these two values. Therefore, since this $3 \mathrm{D}$ model only approximates the actual sculpin, the base-noise threshold will be set to $0.075 \mathrm{~Pa} / \mathrm{m}$, which likely stimulates three canal neuromasts.

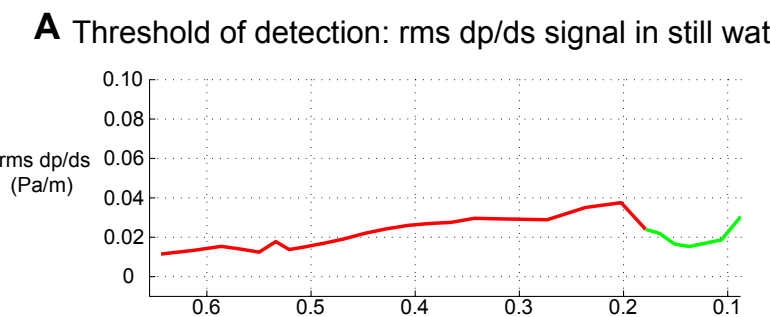

\section{B Location of best signal}
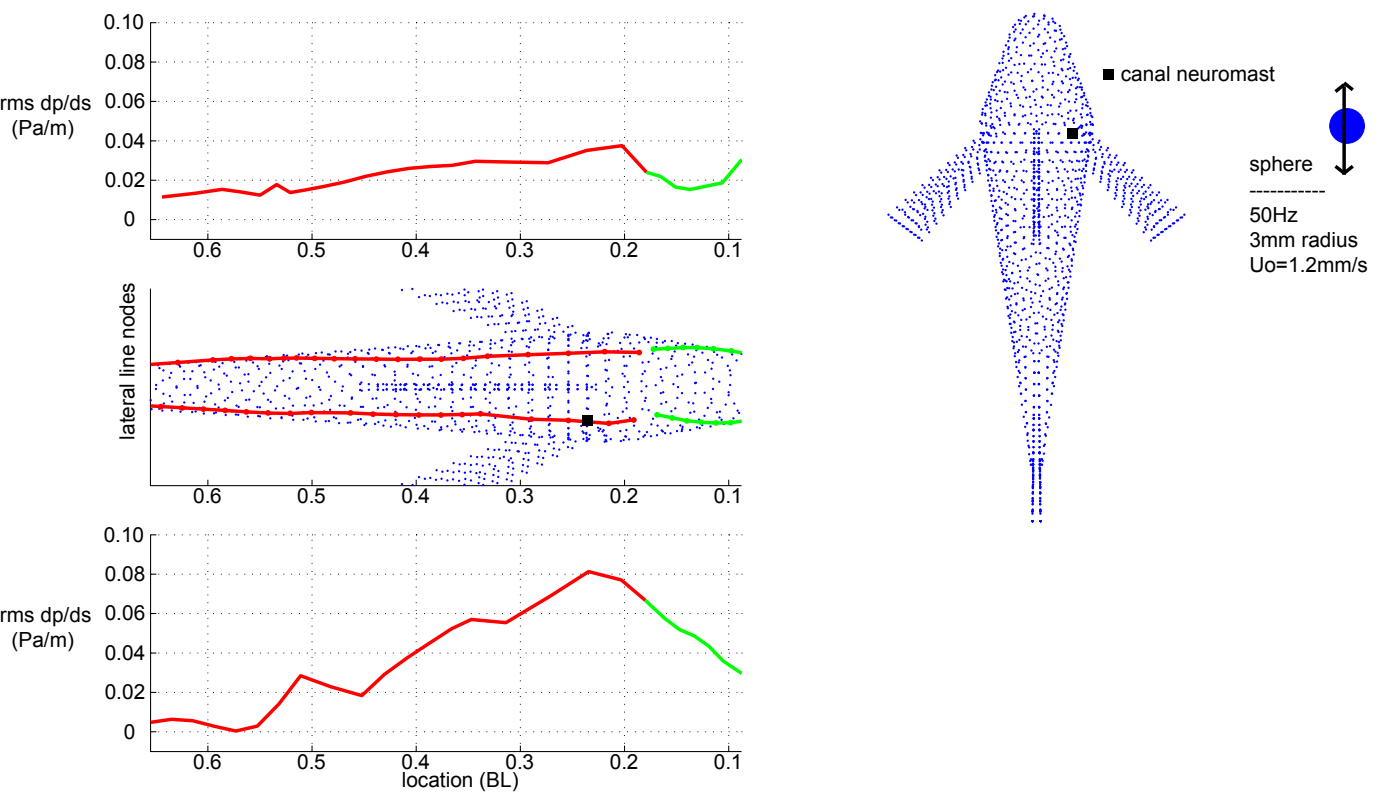

Figure 5-1: The threshold of detection in still water determines the base-noise level. (A) The root mean square (rms) pressure gradient signals along the trunk and infraorbital canal lateral lines due to an oscillating sphere. (B) The sphere is located $5.5 \mathrm{~cm}$ away from the longitudinal midline of the fish, and oscillating in a direction parallel to the midline axis of the fish. The sphere has a radius of $3 \mathrm{~mm}$, oscillates at $50 \mathrm{~Hz}$, and has a velocity amplitude of $U_{0}=1.2 \mathrm{~mm} / \mathrm{s}$ at the threshold of detection, as determined by Kanter and Coombs (2003). Note: in their work they use peak-peak values for amplitudes, while all amplitudes in this thesis are defined relative to peak values ( $=1 / 2$ peak-peak values).

All other canal neuromasts have stimulations which fall below this threshold. It does not mean that that the other canal neuromasts are not moving, but that their motions are small enough to be indistinguishable from the base-noise level inherent 
in the hair cells. For detection to occur these at locations, the velocity amplitude of the sphere would need to be increased from its base level of $1.2 \mathrm{~mm} / \mathrm{s}$. From the 3D still water chapter, we know that at a fixed frequency, the pressure gradient signal scales linearly with the sphere velocity amplitude, $U_{0}$. For each canal neuromast to individually meet the detection threshold level, the velocity amplitude would have to be increased according to

$$
\text { Velocity amplitude threshold }=1.2 \mathrm{~mm} / \mathrm{s} *\left(\frac{\text { detection threshold level }}{\mathrm{rms} \mathrm{dp} / \mathrm{ds} \text { still water level }}\right)
$$

where detection threshold level is defined by Hypothesis I, and the rms dp/ds still water level is defined individually for each neuromast in figure 5-1.

In still water conditions, the canal neuromasts closest to the sphere are the ones that meet the detection threshold level. However, in moving water conditions, the detection threshold level for each neuromast will change, and it could be that some locations become much noisier than others. The relative increase in velocity amplitude might be less at a location further away, because the detection threshold level is much lower. This will be dependent on the fish-prey orientation and flow conditions.

\subsubsection{Mean flow characteristics}

The average velocity and vorticity for the sculpin with fins extended and retracted in laminar flow is presented in figures 5-2 and5-3. The free stream velocities are set at 2,4 , and $8 \mathrm{~cm} / \mathrm{s}$, though at the level of the measurements $(1.1 \mathrm{~cm}$ above the floor), the boundary layer can interact to reduce these speeds. The sphere is located $1.6 \mathrm{~cm}$ above the floor and to the side of the fish (see figure 5-1B), and so does not appear in these figures. That affects of the sphere presence, in alterations of the flow around it, can be seen more easily in the vorticity plots. These plots are averaged at $200 \mathrm{~Hz}$ over a time interval of $2 \mathrm{~s}$.

Figure 5-2 shows that a boundary layer of reduced flow develops around the side of the fish, and grows pronounced after rounding the sides of the head. This is similar to what was observed for the blind Mexican cave fish while in its gliding maneuvers 
(Windsor, 2008). This type of boundary layer is not expected in swimming fish, who are able to keep the boundary layer attached to its surface (Anderson et al., 2001).

Figures 5-3 and 5-3 shows the flow around the sculpin body when it has its fins extended. These results show qualitative agreement with the Coombs et al. (2007) results, especially figure 5-3 where turbulence is introduced. There is a significant reduction in flow in the region behind the extended pectoral fins. The fins are tilted, which acts to help keep their bodies pushed to the floor in increasing rates of background flow. There are regions of recirculation behind the fins in both the laminar flow and turbulent flow cases, and shedding also occurs for the turbulent flow case.

The flow moving past the body will stimulate both the superficial and the canal neuromasts. For this chapter only the canal neuromasts are considered. As the flow moves past the body, it will accelerate as it is forced around the sides of the head, and decelerate on the other side. Flow accelerations indicate the presence of pressure gradients, which are the signal to the canal lateral line system. Figure 5-5 shows a comparison of how this signal changes as flow is increased for both the body with fins retracted and extended. The plots present the instantaneous pressure gradient signal to the trunk and infraorbital lateral lines, sampled over 2 s at $10 \mathrm{~Hz}$. Plots are presented in normalized units, and the direction of the positive pressure difference between canal pore openings is in the direction of the arrows on the side of the fish. The arrow direction is arbitrary, and it means that a positive value indicates that the flow in the lateral line canal is in the direction of the arrow, and a negative value indicates that flow in the canal is in a direction opposite the arrow.

The signal that appears towards the nose of the fish is a combination of the two classic pressure gradient patterns that appear for a dipole source oscillating parallel and perpendicular to a wall (see figure 2-4). The reason is that at the nose of the fish the flow is forced to a stop, creating a high pressure point. As the flow moves away from the nose it accelerates, reaching its fastest speeds rounding the side of the head and creating a pressure low on both sides of the fish. The canal lateral line samples this pressure field, which appears as a parallel dipole source from the nose towards the side of the head, but then appears as a dipole source oscillating perpendicular to 
Average laminar flow characterisitics

Velocity magnitude $(\mathrm{cm} / \mathrm{s})$
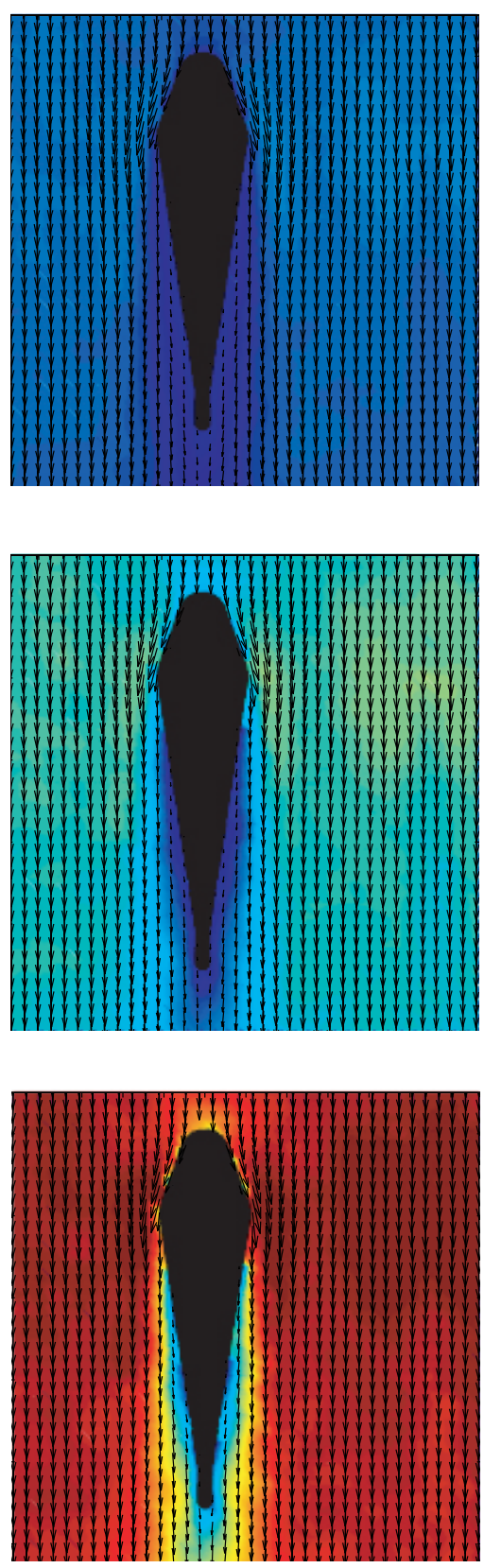

Vorticity $(1 / \mathrm{s})$
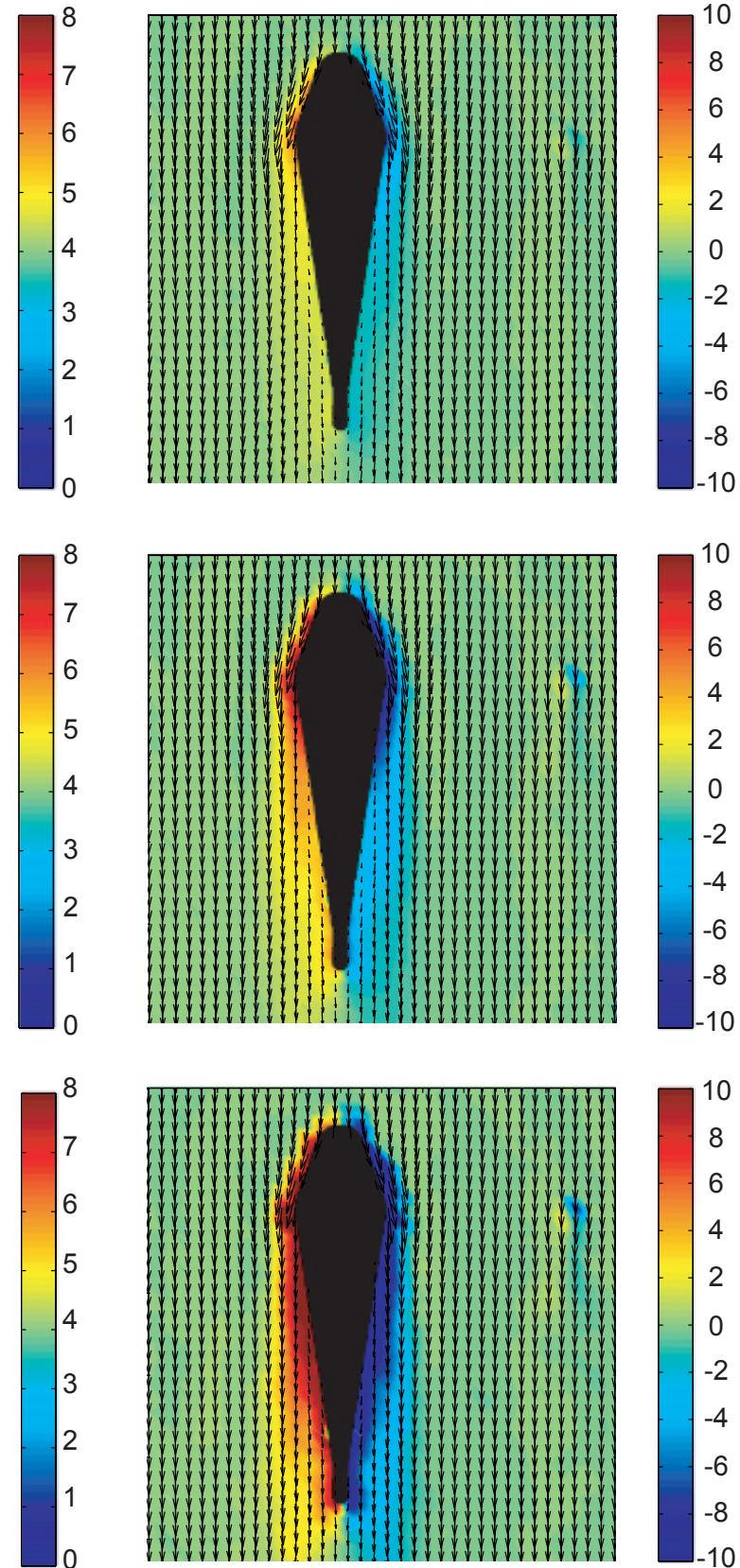

Figure 5-2: Average laminar flow characteristics for sculpin with retracted fins. The free stream velocities are 2,4 , and $8 \mathrm{~cm} / \mathrm{s}$. Measurements of velocity and vorticity were sampled at $200 \mathrm{~Hz}$ for $2 \mathrm{~s}$, at locations $1.1 \mathrm{~cm}$ above the floor. There is a region of reduced flow in the boundary layer that grows along the sides of the fish. 
Average laminar flow characterisitics

Velocity magnitude $(\mathrm{cm} / \mathrm{s})$
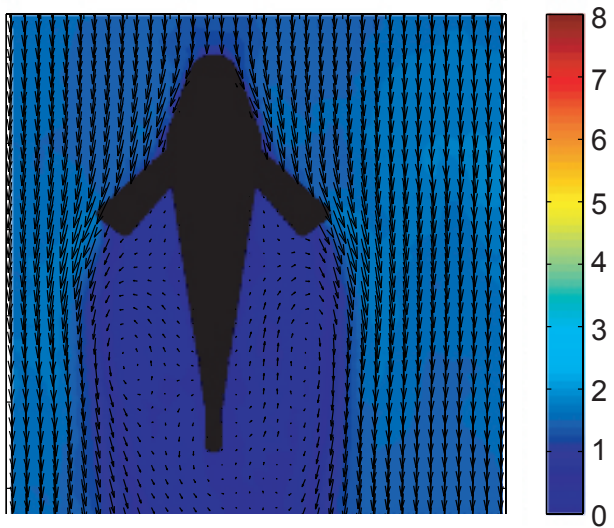

Vorticity $(1 / \mathrm{s})$
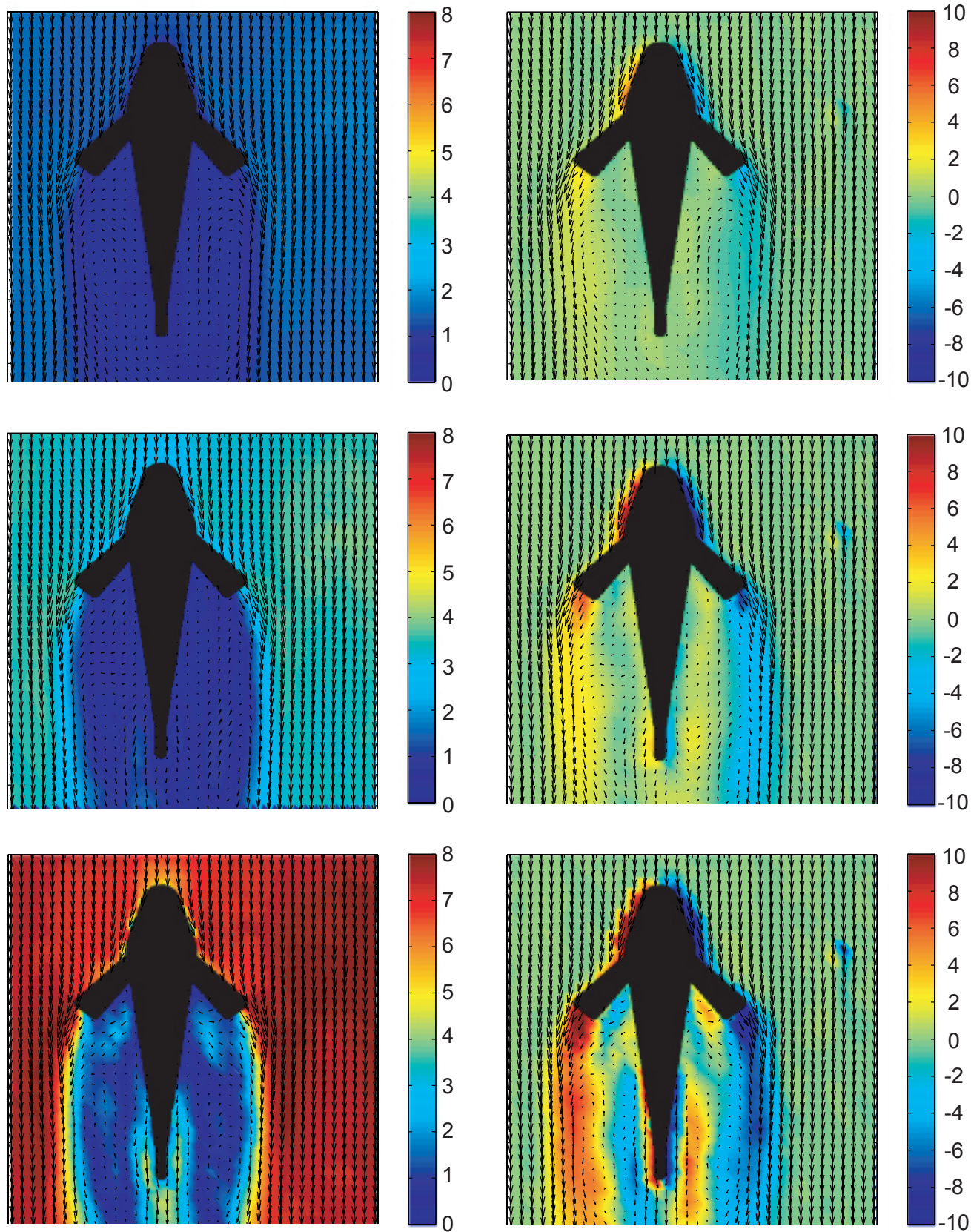

Figure 5-3: Average laminar flow characteristics for sculpin with extended fins. The free stream velocities are 2,4 , and $8 \mathrm{~cm} / \mathrm{s}$. Measurements of velocity and vorticity were sampled at $200 \mathrm{~Hz}$ for $2 \mathrm{~s}$, at locations $1.1 \mathrm{~cm}$ above the floor. There are regions of reduced flow and recirculation behind the pectoral fins. 
Average turbulent flow characterisitics ( $\mathrm{rms}$ vel $2-4 \mathrm{~mm} / \mathrm{s}$ )

Velocity magnitude $(\mathrm{cm} / \mathrm{s})$
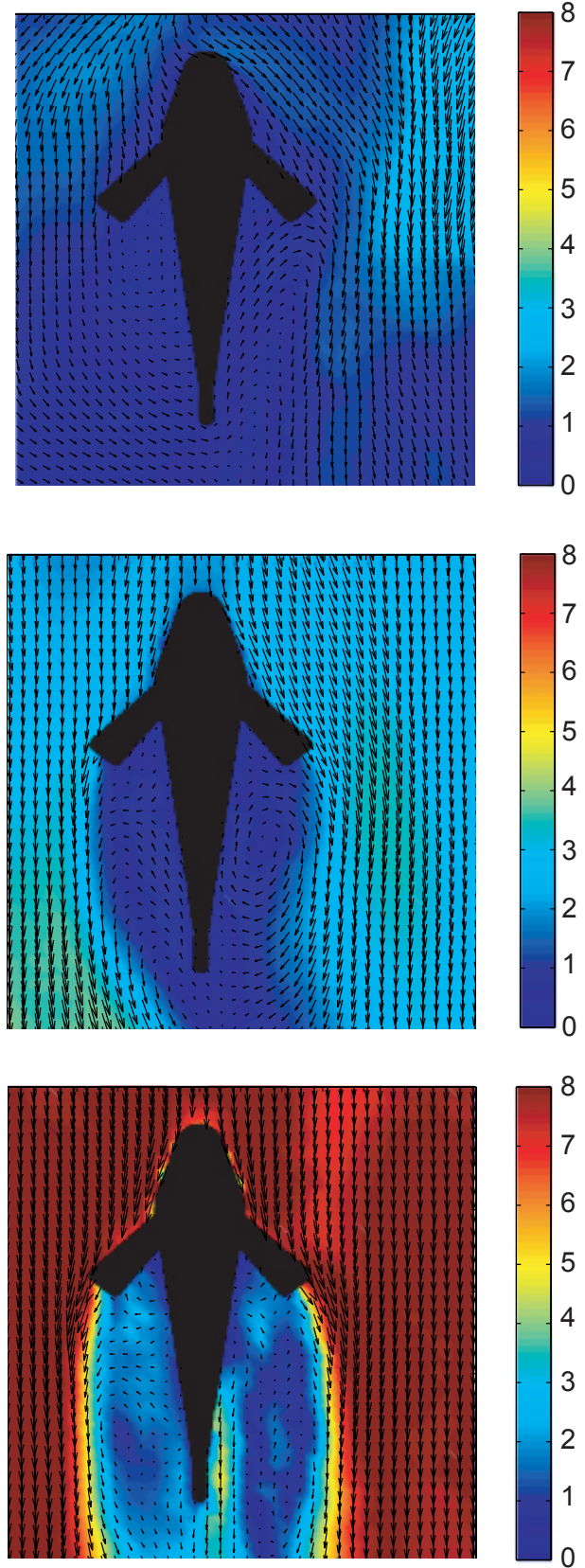

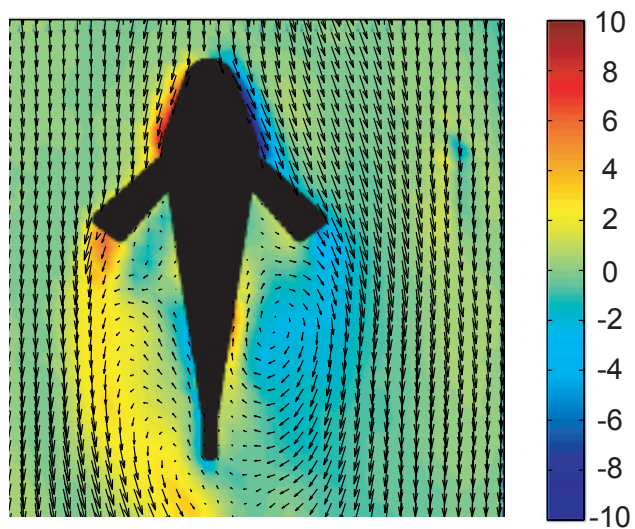

Vorticity $(1 / s)$
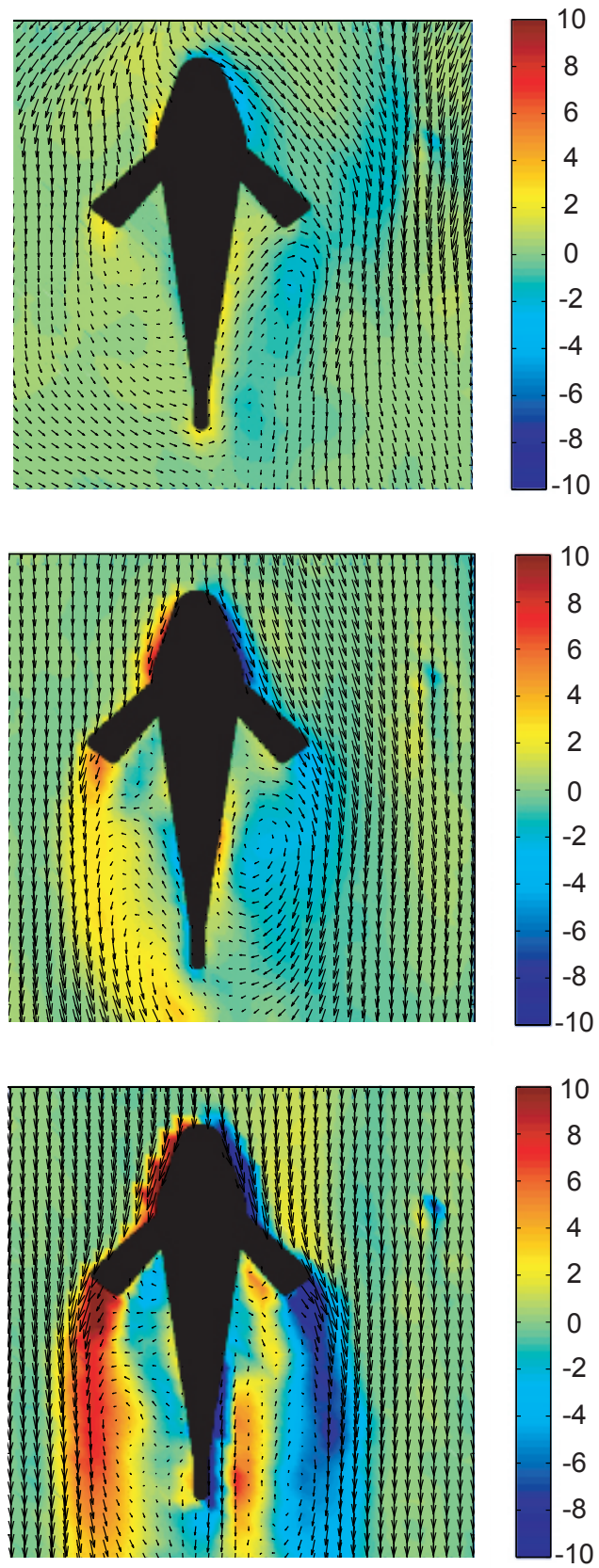

Figure 5-4: Average turbulent flow characteristics for sculpin with extended fins. The free stream velocities are 2,4 , and $8 \mathrm{~cm} / \mathrm{s}$. Measurements of velocity and vorticity were sampled at $200 \mathrm{~Hz}$ for $2 \mathrm{~s}$, at locations $1.1 \mathrm{~cm}$ above the floor. Turbulence was introduced $30 \mathrm{~cm}$ upstream of the fish, and characterized in the region of the fish. There are regions of reduced flow and recirculation behind the pectoral fins, in addition to shedding events around the tail. 
the fish as it rounds the head. This is the prominent signal.

Hassan (1992a) used potential flow theory to compute the normalized pressure differences along axisymmetric bodies gliding through the water, and having various shapes and widths. The signal is measured around the edge of a 2D slice through the body, and shows a similar range of maximum values up to 0.30 . The signals do not capture the classic dipole pressure gradient patterns seen here for two reasons. The first reason is that the lateral line of the sculpin curves up and around the pectoral fins, and samples the pressure field in a different manner. The second reason is due to the real effect the viscous boundary layer around the fish which changes the nature of the pressure field around the surface of the fish. This effect was attributed by Windsor (2008) as a low Reynolds number effect, where the boundary layer is more prominent than in high Reynolds number flows. Potential flow theory is often used to approximate high Reynolds number flows, where inertial effects dominate, and viscous effects are kept to a very small boundary layer relative to the size of the object in the flow.

Another important difference between these results and those of Hassan and Windsor is that the maximum values of the normalized pressure gradient signals grow with increasing flow velocity. Potential flow theory, used by Hassan, precludes this possibility. Including the effects of viscosity could affect this result, since the boundary layer on the fish changes with flow velocity. However, Windsor's results show that for fish bodies of axisymmetric shape, the normalized signals overlap at the Reynolds numbers used in the study (1000-8000).

For sculpin, which is a benthic fish, the effects of the floor boundary layer are more significant at lower flow velocities. The pressure gradient signals are normalized by the free stream velocities of 2,4 , and $8 \mathrm{~cm} / \mathrm{s}$. However, as figures $5-2$ and 5-3 show, the flow is reduced from these values around the fish. The height of the sculpin in these simulations is $2 \mathrm{~cm}$, and interacts more directly with the bottom boundary layer at the lower velocities. Normalizing by the velocities present around the sculpin would increase the values seen in the plots for the 2 and $4 \mathrm{~cm} / \mathrm{s}$ flows.

There are also slight changes in the shape of the signals as the flow increases in 
speed, and that suggests that the shape of the body matters. The sculpin body is not axisymmetric. Additionaly, the 3D model of the sculpin includes a dorsal fin, a tail, and pectoral fins (when extended). There are regions of isolated noise that appear at different places along the lateral line at different flow speeds, depending on how the body and flow are interacting over this observed period of time.

The pressure gradient patterns differ very little for the situation with pectoral fins extended or retracted. It seems like the placement of the lateral line, up and around the pectoral fins, negates some of the effects of the fins presence. One effect that can be seen is a decrease in the maximum values obtained for the signals at each flow speed, and on each side of the body. The fins redirect the flow in a manner that mildly evens out the pressure changes.

When turbulence is introduced, figure 5-6 shows that signal excursions grow in magnitude, but the overall shape remains the same. The extension of pectoral fins does make a difference now. The region along the trunk lateral line behind the pectoral fins shows much more activity than when the fins are retracted. It appears that the recirculation and shedding events add more noise.

\subsubsection{Neuromast impulse and step responses}

The previous section described the mean flow characteristics to the canal lateral line system. Although the mean component of the signal is often dismissed because of the high pass filtering characteristics of the hair cell system, its importance cannot be underestimated. The neuromasts respond to the hydrodynamic signals impressed upon them, and a mean component will cause a sustained deflection of the neuromast and hair cells contained within. In fact, as the hair cell adapts, it relaxes its stiffness, and bends even further (Howard and Hudspeth, 1987). The neuromast models of van Netten (2006) and Humphrey et al. (2001) adequately describe the canal and superficial neuromast responses to local fluid velocities, respectively. This section explores the responses of the canal neuromast in more detail, in particular, to impulse, step, and oscillatory signals.

Curcic-Blake and van Netten (2005) have measured and modeled the responses of 
Instantaneous self flow pressure gradient signal, sampled at $10 \mathrm{~Hz}$ for $2 \mathrm{~s}$, in laminar background flow
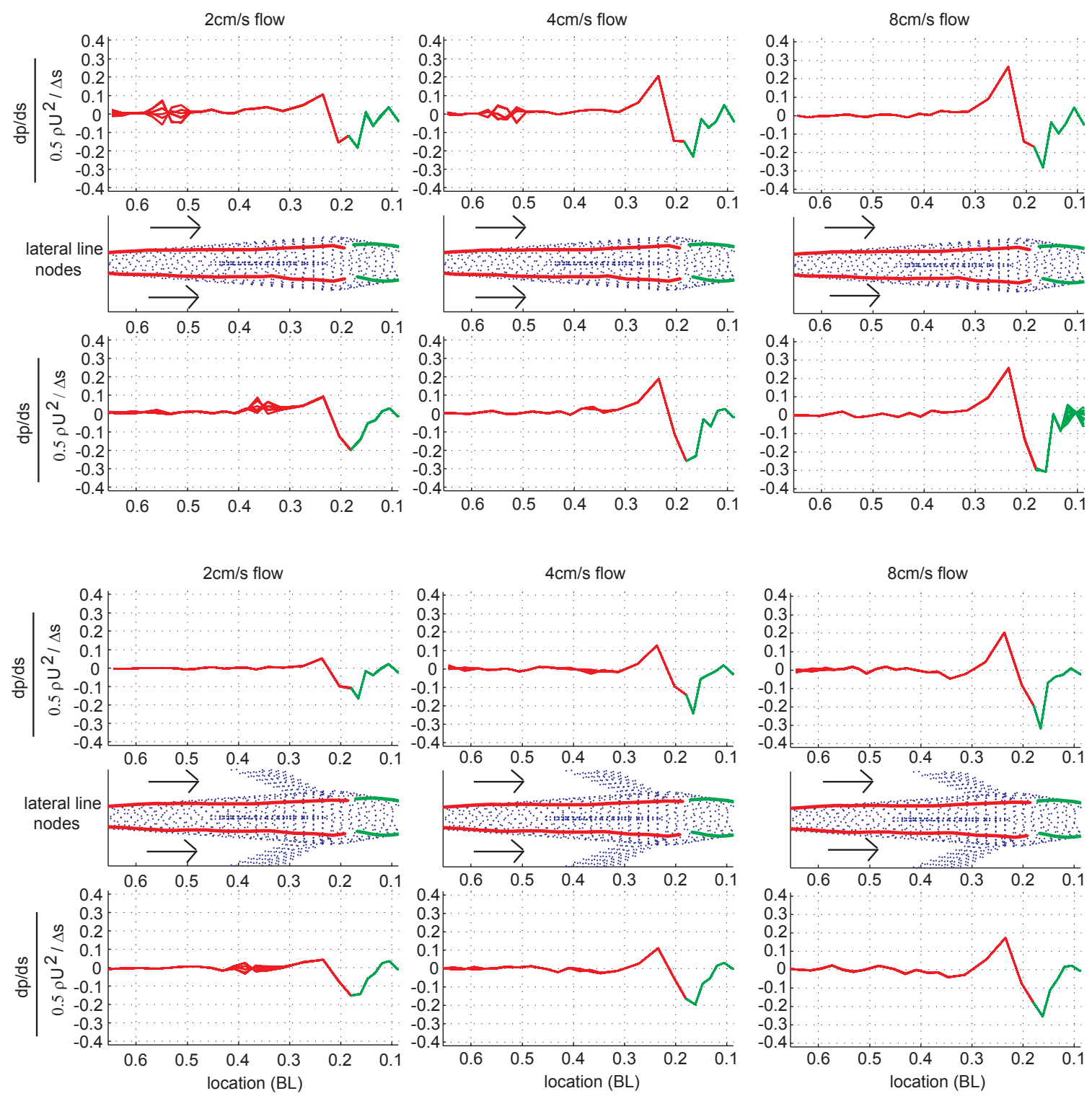

Figure 5-5: Instantaneous normalized pressure gradient signals along the infraorbital and trunk lateral lines. The flow is laminar, and increasing in speed from $2-8 \mathrm{~cm} / \mathrm{s}$. Data is sampled at $10 \mathrm{~Hz}$ over $2 \mathrm{~s}$. The fish bodies are placed in the middle of each plot, and indicate the locations of the canal lateral line pore openings. The presence of the dorsal fin is visible as the darkened strip down the central portion of the fish, and the extended pectoral fins are also evident. The arrows indicate the pressure difference convention used. Positive values indicate that flow within the canals is in the direction of the arrows. The canal pore spacing, $\Delta s$, is $2 \mathrm{~mm}$. 
Instantaneous self flow pressure gradient signal, sampled at $10 \mathrm{~Hz}$ for $2 \mathrm{~s}$, with increasing levels of turbulence
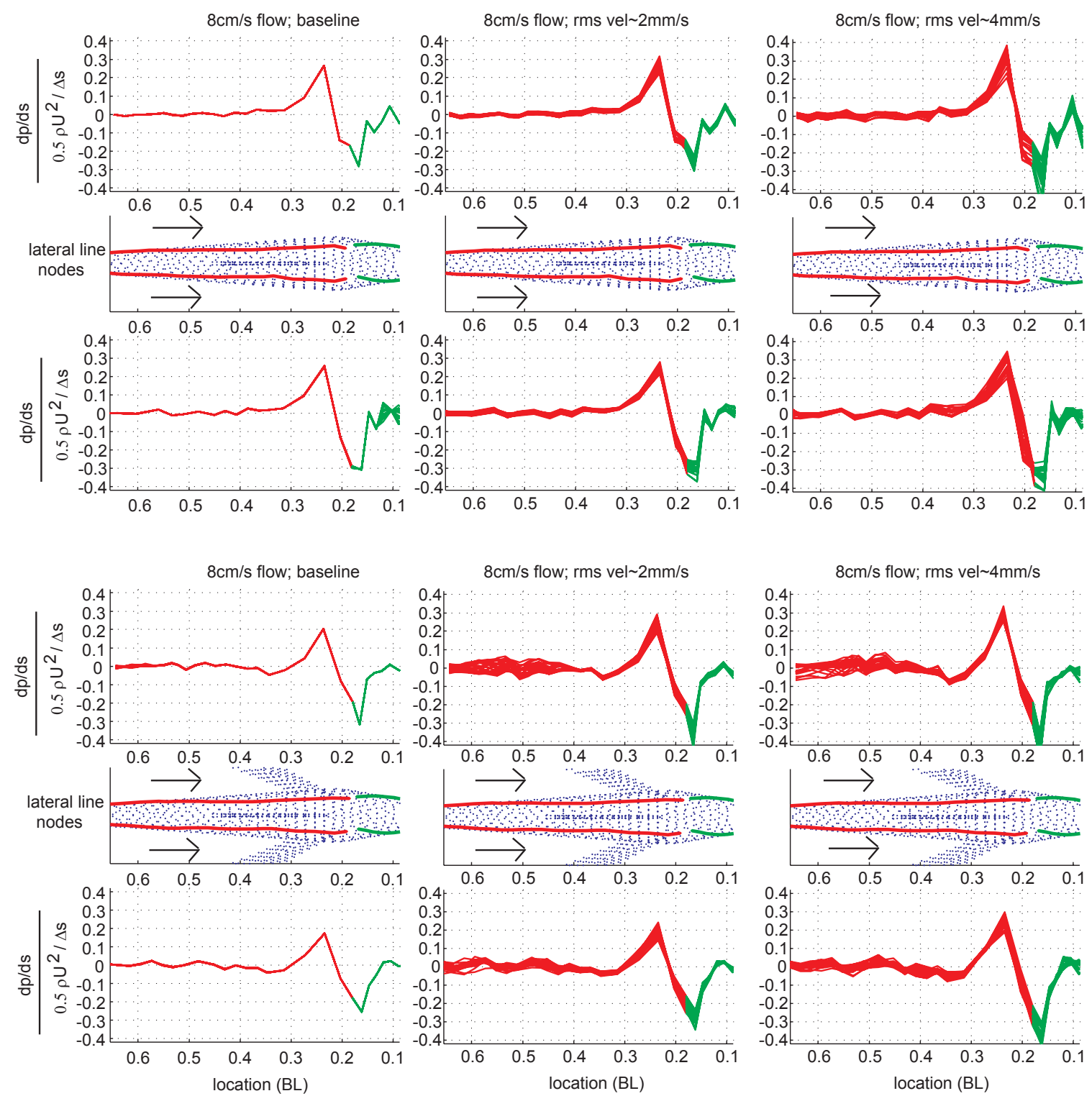

Figure 5-6: Instantaneous normalized pressure gradient signals along the infraorbital and trunk lateral lines. The flow is set at $8 \mathrm{~cm} / \mathrm{s}$, and the level of turbulence is increased from laminar flow to flow with rms velocity values of $4 \mathrm{~mm} / \mathrm{s}$ (mean velocities subtracted), as measured at $1.1 \mathrm{~cm}$ above the floor around the fish. Data is sampled at $10 \mathrm{~Hz}$ over $2 \mathrm{~s}$. The arrows indicate the pressure difference convention used. Positive values indicate that flow within the canals is in the direction of the arrows. The canal pore spacing, $\Delta s$, is $2 \mathrm{~mm}$. 
the supraorbital canal neuromasts of the ruffe fish to impulse responses. Equations 3.4, 3.5, and 3.6 model their results for the canal neuromast response to local fluid motions. Figure 5-7 (top) shows the neuromast response to an impulse and step response. The impulse signal is actually a constant velocity signal of $1 \mathrm{~ms}$ duration, to match the displacement motion of Curcic-Blake and van Netten's impulse signal. The two parameters are the resonance number, $N r=69$, and the transition frequency, $f t=16 \mathrm{~Hz}$. Canal neuromasts for any given fish have a range of values for these two parameters, each modeling changes in the neuromast response. The shape of the impulse response displays the mechanical filtering properties of the neuromast itself, and subtle changes result in different filtering properties. This characterization process is the time analog to the white spectrum, reverse correlation method described by Fay (1987).

The measured results of Curcic-Blake and van Netten differ slightly from the modeled results. They attribute this to the nonlinear gating compliance effects, which are most prominent at the moment of the impulse. As time increases, the stiffness properties of the hair cells change, causing slightly faster cycles than predicted by the linear model. Nonetheless, the differences are very slight, showing that the hair cell mechanics only mildly affect the overall neuromast motion. This strengthens the idea that motion of the neuromast can be modeled separately from the hair cell synaptic responses, though the motion influences significantly the hair cell responses.

The shape of the impulse response determines not only the mechanical filtering properties, but determines how it will respond to other types of signals, such as the step response. This is most clearly observed in figure 5-8 (top), which shows the impulse and step responses of the sculpin canal neuromast models (see also figure 315 and subsequent modifications for model parameters). Variations in the maximum excursions, as well as the time between zero crossings, will affect the final steady state step response. The steady state step response indicates neuromast displacement (deflection) to the mean part of the signal.

The local fluid velocity within the subdermal canal has a frequency dependent solution based on the boundary layer height that develops (see figures 3-7 and 3-8). 
It is the pressure difference between canal pore openings that drives the fluid within the canal, and so the overall response of the canal neuromast to outside water motion has to be taken into account. Figures 5-7 and 5-8 (bottom) show the canal neuromast impulse and step responses to outside acceleration.

Figure 5-9 shows the sculpin canal neuromast response to sinusoidal signals. Two different frequencies are used $(20 \mathrm{~Hz}$ and $50 \mathrm{~Hz})$ to demonstrate that although the neuromast is more sensitive to particular frequencies of local fluid motion (top), the overall system response to outside acceleration signals is fairly constant (bottom). This was main point of van Netten (2006) in describing how sensitivity to particular frequencies, due to inertial resonance properties of the neuromast, often balances the filtering effects of the canal channel, yielding an overall flat system response. For the sculpin models presented, the spherical and flat plate models respond very similarly to the sinusoidal signals (unlike the step responses), while the cylindrical model is overall more sensitive.

The fact that fish neuromasts have a variety of impulse responses suggests that each may be tuned to respond to environmental signals in a particular way. Each neuromast on the fish body will encounter different hydrodynamic signals based on its location on the body and the type of signal present. To illustrate this concept, figure 510 shows the resulting neuromast motions to oncoming flow for three locations on the sculpin body. The flow is laminar upstream, moving at $8 \mathrm{~cm} / \mathrm{s}$, and 2 seconds worth of the pressure gradient signal is given for a point by the nose (on the infraorbital lateral line), a point by the pectoral fin insertion location (trunk lateral line), and a point by the tail (trunk lateral line). The neuromast displacement for the three types of neuromast models for the sculpin are compared with the displacements of the canal lateral line of the Ruffe, whose parameters are given in Curcic-Blake and van Netten (2005). On each plot the pressure gradient signal is identified by an arrow to the side of the plot. The oscillations seen in the neuromast motions are mostly from turbulence that develops along the floor of the channel, sides of the fish, and behind the fins. The $50 \mathrm{~Hz}$ oscillations from the sphere cannot be identified (by eye) in these plots. 


\section{Ruffe canal neuromast response to local fluid velocity \\ - Signal \\ - Neuromast - spherical model}
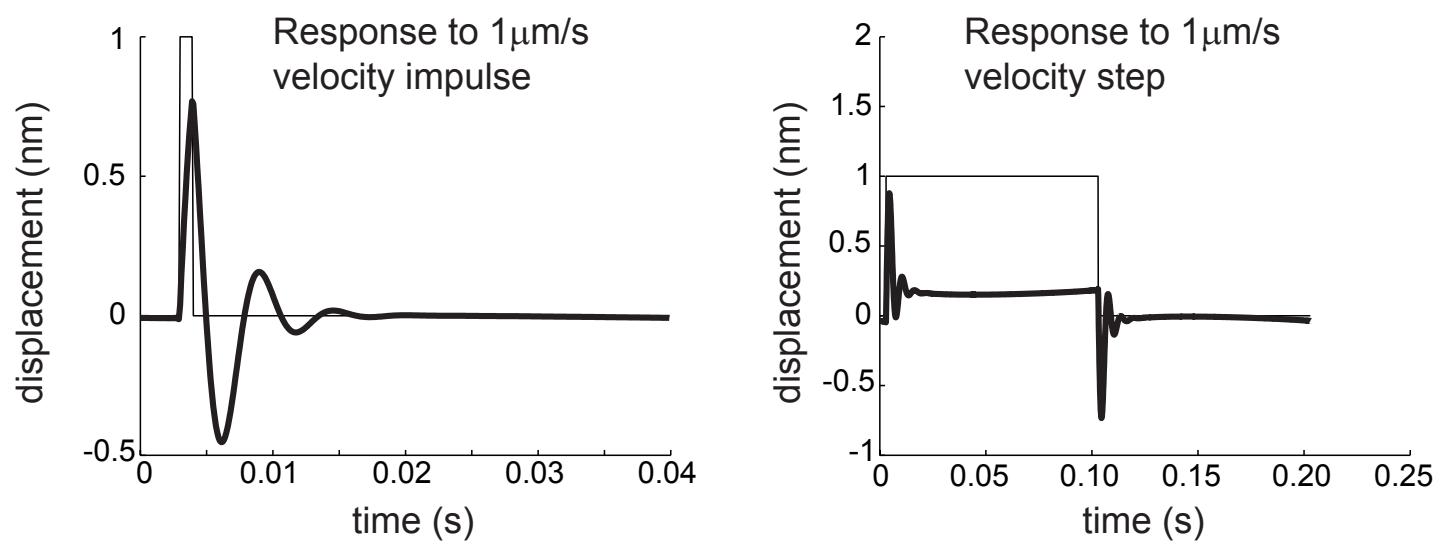

Ruffe canal neuromast response to outside fluid acceleration
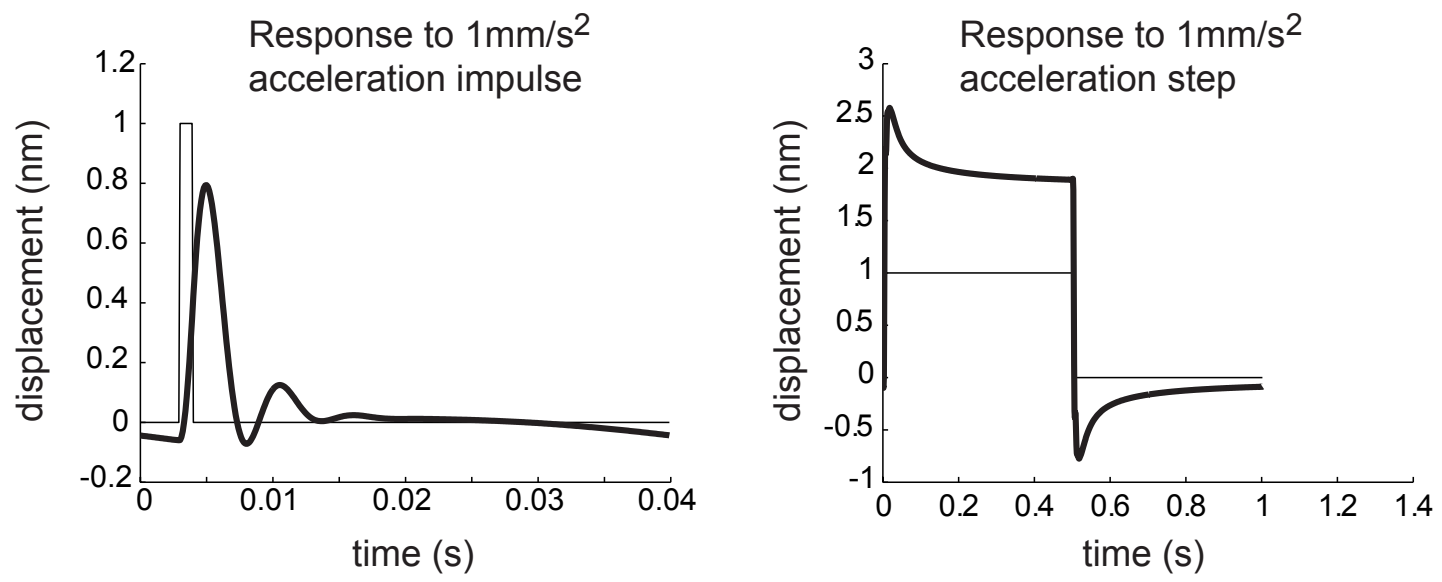

Figure 5-7: Impulse and step responses of the Ruffe canal neuromast. The top plots show the neuromast displacements to impulse and step inputs of $1 \mu \mathrm{m} / \mathrm{s}$ velocity amplitude of the fluid within the canal. The bottom plots show the neuromast displacements to impulse and step inputs of $1 \mathrm{~mm} / \mathrm{s}^{2}$ acceleration amplitude of the fluid outside of the canal. The neuromast model is based on the work of van Netten (2006), and has a resonance number of $N r=69$, and a transition frequency of $f t=16 \mathrm{~Hz}$. For measured impulse responses, see Curcic-Blake and van Netten (2005). 


\section{Sculpin canal neuromast response to local fluid velocity}
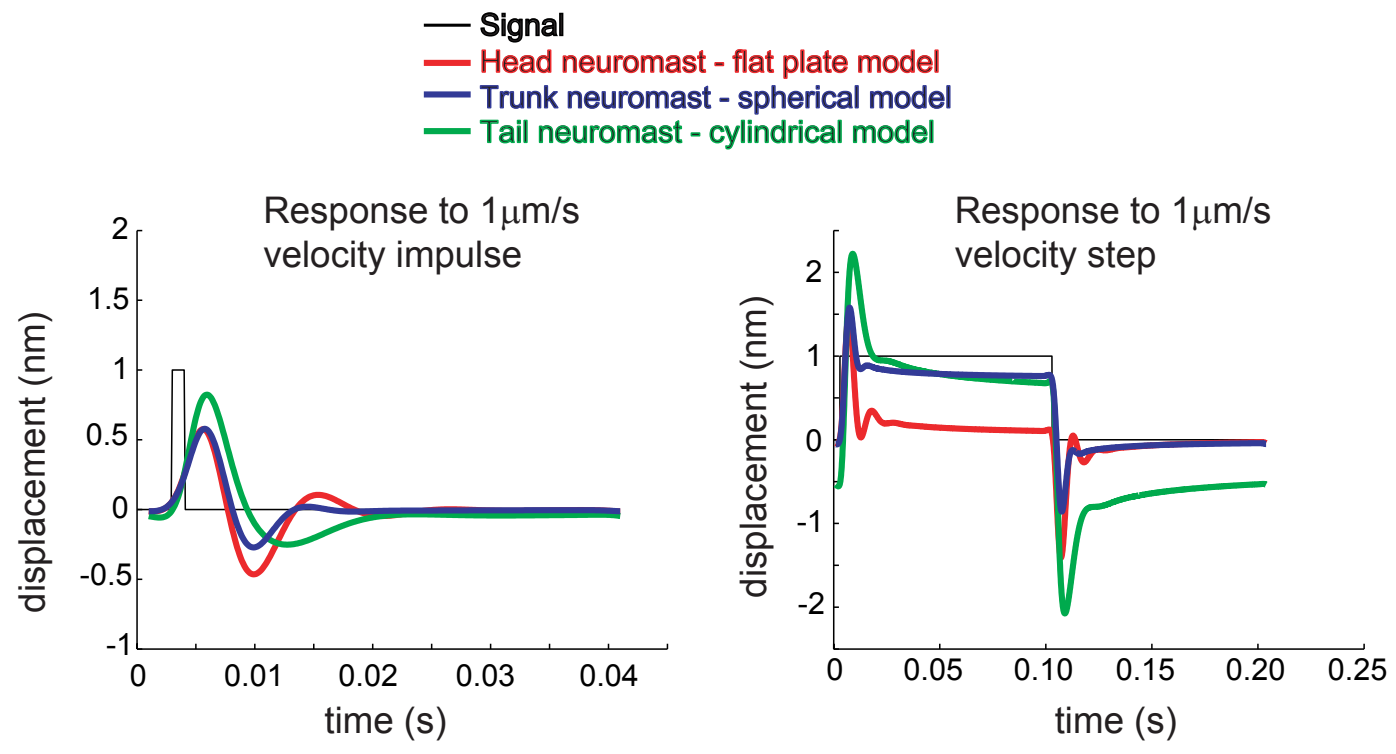

Sculpin canal neuromast response to outside fluid acceleration
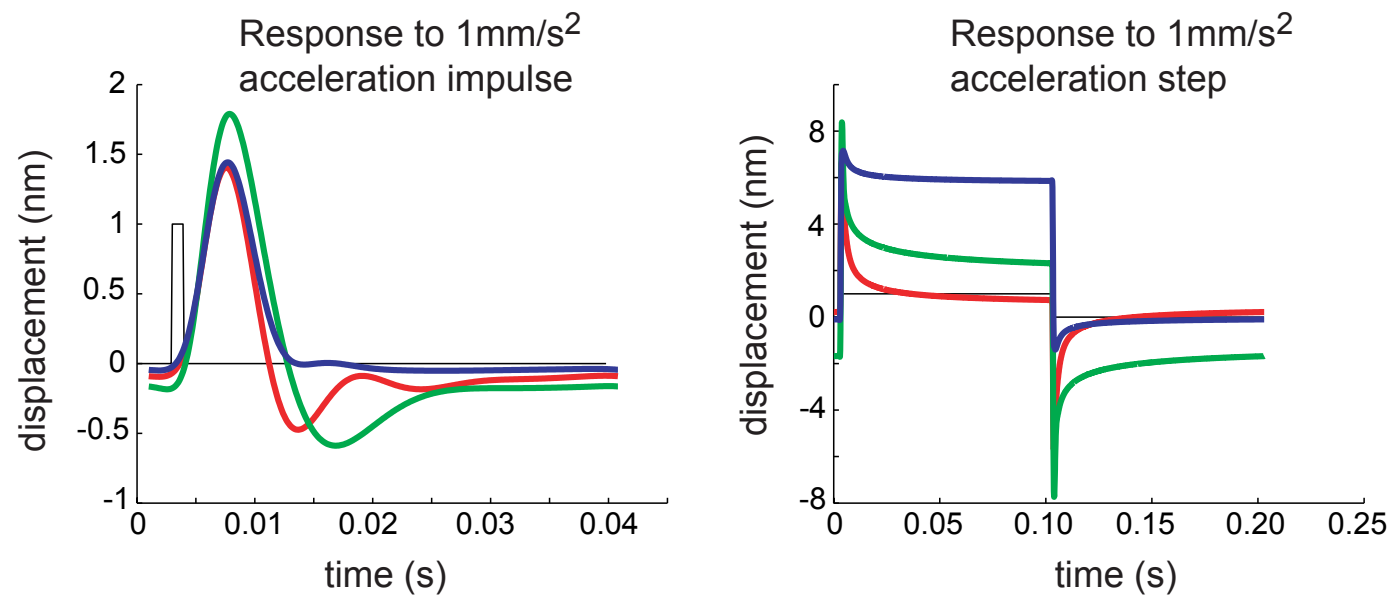

Figure 5-8: Impulse and step responses of the Sculpin canal neuromast. The top plots show the neuromast displacements to impulse and step inputs of $1 \mu \mathrm{m} / \mathrm{s}$ velocity amplitude of the fluid within the canal. The bottom plots show the neuromast displacements to impulse and step inputs of $1 \mathrm{~mm} / \mathrm{s}^{2}$ acceleration amplitude of the fluid outside of the canal. Three neuromast models for the sculpin are compared (see text for details). 


\section{Sculpin canal neuromast response to local fluid velocity}
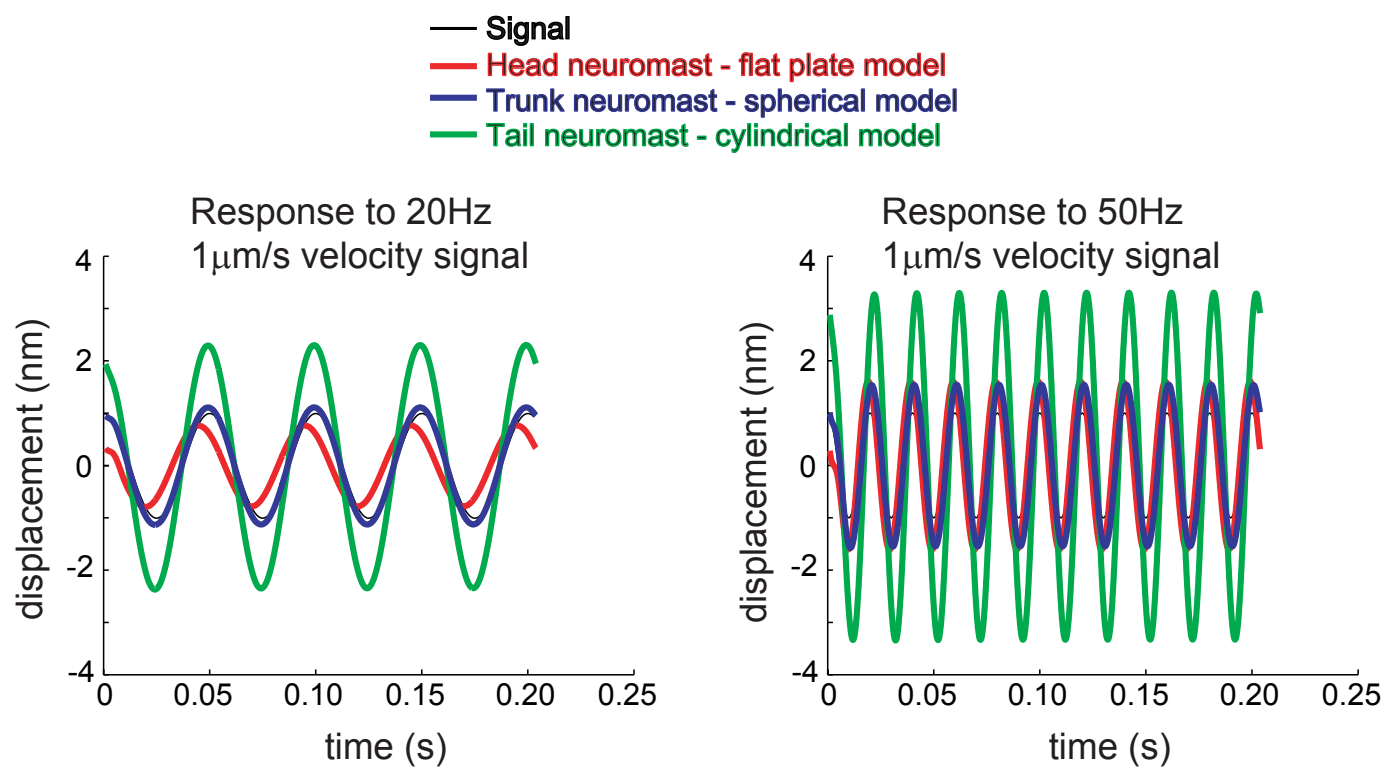

Sculpin canal neuromast response to outside fluid acceleration
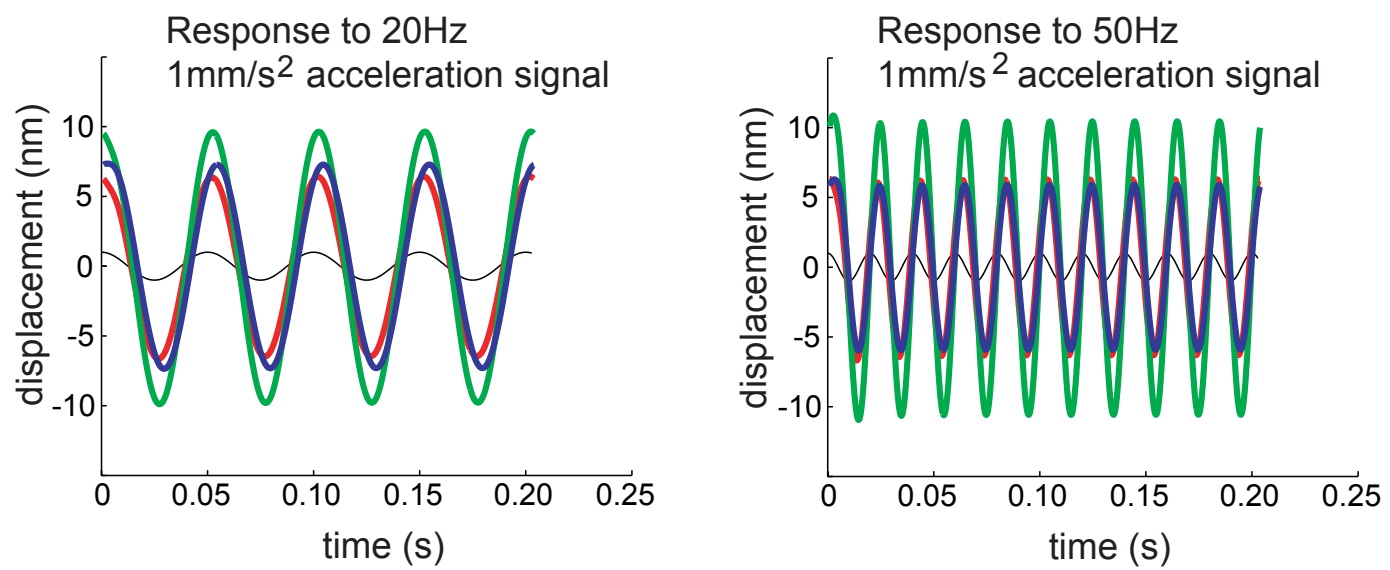

Figure 5-9: Sinusoidal responses of the Sculpin canal neuromast. The top plots show the neuromast displacements to sinusoidal inputs of $1 \mu \mathrm{m} / \mathrm{s}$ velocity amplitude of the fluid within the canal. The bottom plots show the neuromast displacements to sinusoidal inputs of $1 \mathrm{~mm} / \mathrm{s}^{2}$ acceleration amplitude of the fluid outside of the canal. Three neuromast models for the sculpin are compared (see text for details). 


\section{Comparison of canal neuromast model responses to oncoming flow}
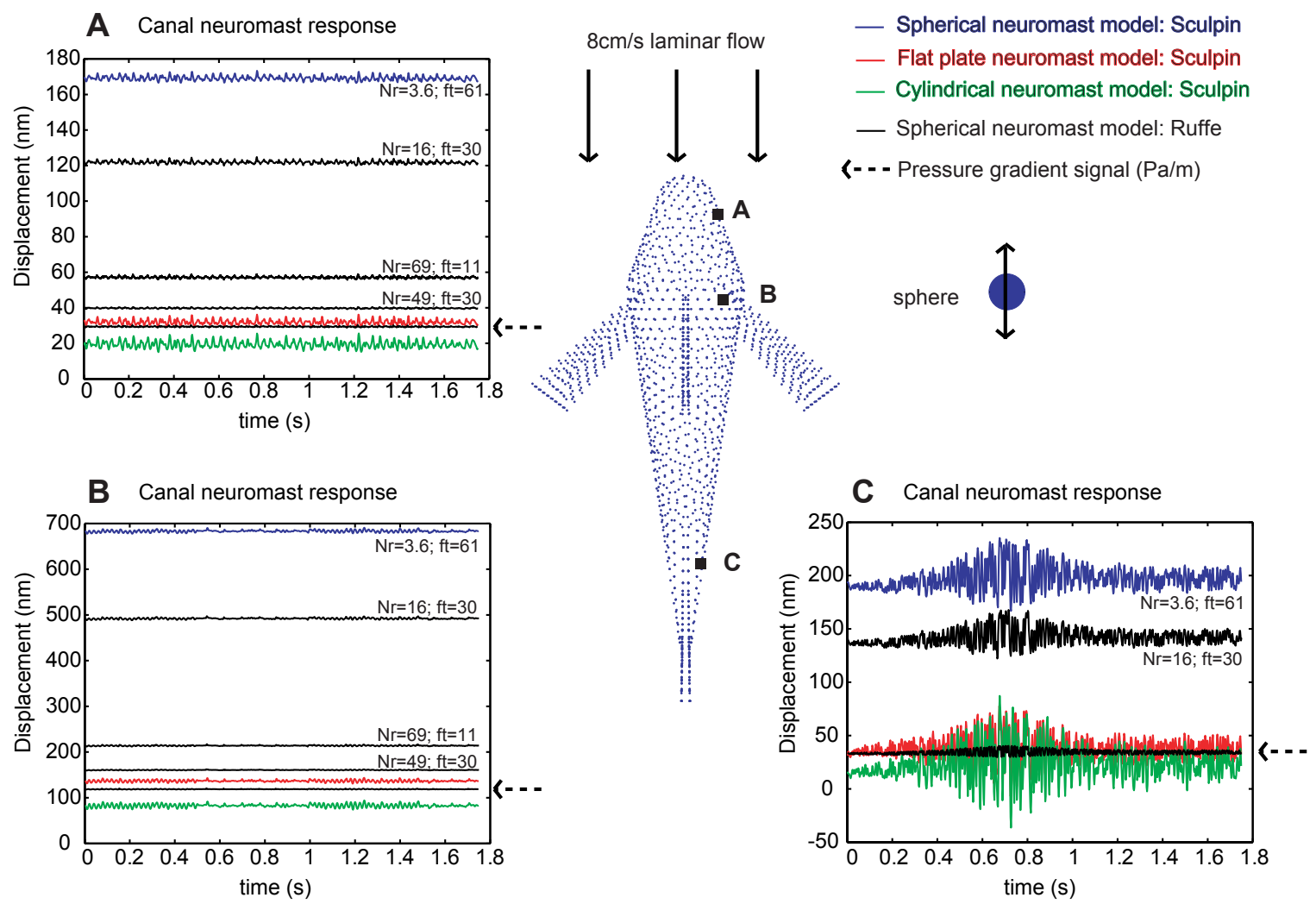

Figure 5-10: Neuromast model comparison in $8 \mathrm{~cm} / \mathrm{s}$ laminar flow . Three neuromast models for the sculpin (see text), and one model for the Ruffe (van Netten, 2006), are compared at three separate locations on the sculpin body (labeled A, B, and C). The resonance number, $N r$, and transition frequency, $f t$ (in $\mathrm{Hz}$ ), for the spherical neuromast models are listed next to each response. The pressure gradient signal in each plot is identified by the arrow to its side. The units on the ordinate represent displacement motions of the neuromast $(\mathrm{nm})$, and $\mathrm{Pa} / \mathrm{m}$ for the pressure gradient signal only. The location of the sphere is shown relative to the fish body. The oscillations seen in the neuromast motions are mostly from turbulence that develops along the floor of the channel, sides of the fish, and behind the fins. The $50 \mathrm{~Hz}$ oscillations from the sphere cannot be identified (by eye) in these plots. 
There are three main observations from these plots. First, the neuromast displacement in response to the mean pressure gradient signal varies greatly by impulse response. This indicates that some neuromasts will be more sensitive to changes in bulk flow conditions than others. Second, the magnitude of displacement depends on specific location of the neuromast on the fish body. Near the nose and tail the pressure gradient is less than above the pectoral fin insertion point. This may be of less consequence than how much noise there is at each location, which is the final observation. The nose being near the floor experiences turbulence from the bottom boundary layer, and the tail experiences turbulence from the flow developed along the body and as a result of flow around the fins. Of the three points chosen, the location of least noise appears to be above the pectoral fin, where any surface boundary would be negligible, and yet is either sheltered from or is above floor induced turbulence.

It should be noted that the neuromast displacements are predicted by linear models. It is unclear to what range of displacement these models are valid. A displacement of $1000 \mathrm{~nm}$ (1 micron) is still small compared to the average size of canal neuromasts (hundreds of micrometers), and so it is likely that the models are valid. However, the material stiffness of individual hair cells do change and in a time dependent nature, and so it would not be surprising to see actual neuromast displacements which differ from those predicted by these models.

It was mentioned earlier that it is the time dependent, oscillatory motion of the hair cells that results in the tensioning and relaxing of the gating apparatus. This would seem to indicate that the mean displacement is irrelevant, because after the initial displacement, the gating apparatus adapts to the hair cells new location. But there is evidence that the hair cells have limits to their ability to adapt. Eactock et al. (1987) show that hair cells adapt almost completely for small amplitude signals (up to $200 \mathrm{~nm}$ ), but increasing less for larger displacements (66\% for an $800 \mathrm{~nm}$ step). Therefore, increasing levels of flow will eventually force all types of neuromasts to respond, as seen in the experiments of Chagnaud et al. (2008). 


\subsubsection{Determination of the high pass characteristics}

Though the material properties of the neuromast, its mass, stiffness, shape, and size, determine how it responds to hydrodynamic stimuli, it is the hair cell itself which determines what signals it will send to the brain. Eatock (2000) reviews this process in detail, explaining how Ca2+ works to help reposition elastic links between the stereocilia to relieve tension, and lower the transducer current. There are additional mechanisms at work within the hair cell, resulting in a process that is not linear. Analytical models which describe the various facets of the hair cell were put forth by Tinevex et al. (2007), and a virtual (numerical) 3D hair model was created by Nam et al. (2007) to duplicate more realistically the multiple degrees of freedom present in real hair cells.

Roberts and Rutherford (2008) give an explanation of the overall purpose and operation state of the hair cell. Basically, they suggest that the hair cell works to actively navigate away from saturation regions (negative and positive), and reach an operating point were responses to small signals are linear. In addition, there are active force generators and electrical amplifications which keep the hair cell near the point of instability, keeping it sensitive to small signals. The combination of these mechanisms provides both range and sensitivity.

For this thesis, there is a more simplified approach to take. An input-output transfer function can serve the purpose of representing the hair cell internal workings. Eatock et al. (1987) provide such a possibility in their experiments on the otolith of a bullfrog's sacculus. In one experiment, they manually displace the otolith using a step function generator, and record the microphonic currents that result. The displacement is not due to fluid passing by, but is a result of actual displacement, and so the output transducer current is a result of the hair cell adaptation to this displacement. The result is an exponentially decaying function with a time constant of $30 \mathrm{~ms}$.

When individual hair cells were manually displaced according to a step function stimulus, the resulting transducer currents had exponentially decaying functions with 
time steps ranging from 10-200 ms, and reaching steady state levels that were $5-80 \%$ of the peak values. Eatock et al. point out that their in vitro experiments are invasive and potentially damaging, and so the results are not definite.

In signal processing terms, the input is a step function, $\mu(t)$, and the output is an exponential decay function, $e^{-t / \tau} \mu(t)$, given as

$$
\mu(t) \rightarrow h(t) \rightarrow e^{-t / \tau} \mu(t)
$$

where $h(t)$ is the time domain transfer function, $t$ is time, and $\tau$ is the time constant. In the time domain, the transfer function is convolved with the step function, but in the frequency domain, it is multiplied. The frequency domain solution, attained by taking Fourier transforms of all functions, is given as

$$
H(\omega)=\frac{j \omega}{1 / \tau+j \omega}
$$

where $\omega$ is the radian frequency. There could also be a nonlinear scaling component based on neuromast displacement, which would appear in the numerator of the transfer function. Secondly, Eatock et al. (1987) have suggested that the response can also be fit by a weighted combination of two exponentials, one with a fast time constant and one with a slow time constant. In the frequency domain, the effects would sum together as $A_{1} H\left(\omega, \tau_{1}\right)+A_{2} H\left(\omega, \tau_{2}\right)$, where $A_{1}$ and $A_{2}$ are the weights.

Figure 5-11 shows some possible output step functions based on the time constant, and the resulting filtering effects. Decreasing time constants result in an increasing range of low frequencies that get filtered, whereas increasing time constants pass more frequencies. In term of scaling, this particular transfer function is not particularly effective in reducing frequencies. Since the plot is in $\mathrm{dB}\left(20 \log _{10}(|H(\omega)|)\right)$, a $20 \mathrm{~dB}$ decrease results in a tenfold reduction of the signal at that frequency, whereas a 6 $\mathrm{dB}$ decrease results in a twofold reduction. At the tenfold reduction mark $(-20 \mathrm{~dB})$, the $10 \mathrm{~ms}$ time constant has reduced frequencies from 0-1.6 Hz, and for the higher time constants, the range lessens to under $1 \mathrm{~Hz}$. It should be emphasized that these filtering effects do not affect the neuromast or hair cell displacement, but do affect 
the synaptic signals that the hair cell sends to the fish brain.
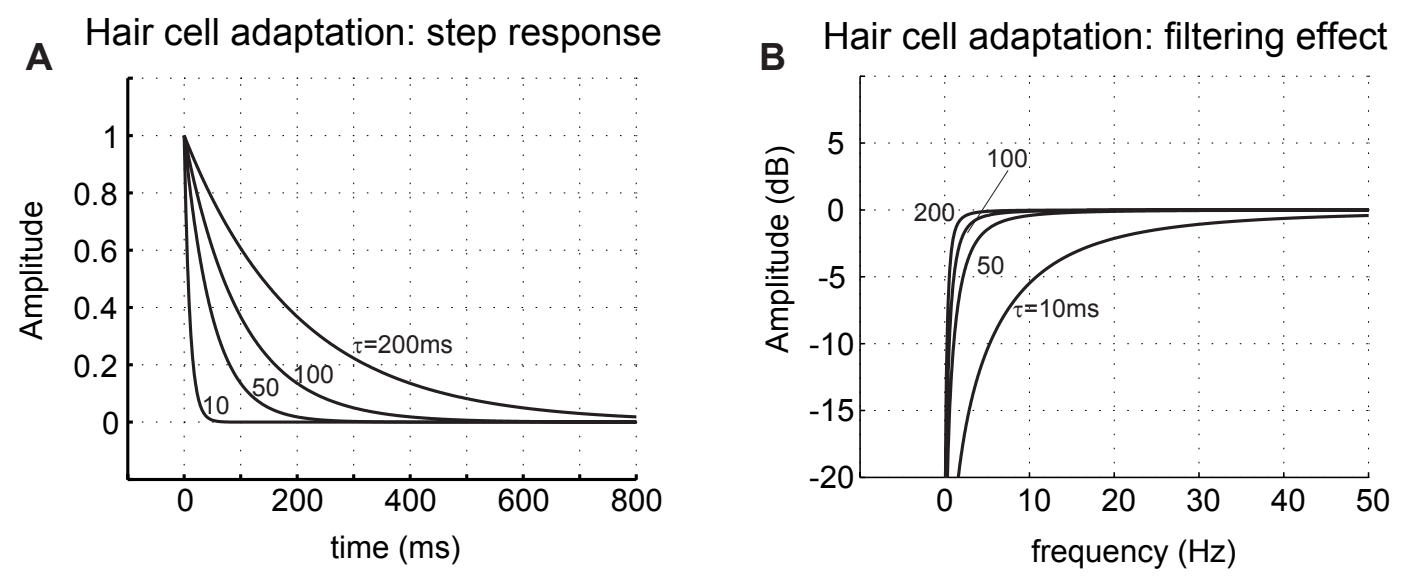

Figure 5-11: Hair cell adaptation and filtering effects. (A) The experimental results of Eatock et al. (1987) suggest that hair cells have an exponential decaying response to a step displacement. Curves are based on a range of time constants, $\tau$, that reflect measured responses. (B) In the frequency domain, the exponential decay step response has filtering properties. Note: this filtering does not apply to the hair cell motion, but to the synaptic signals that it sends to the brain.

For this thesis, the pressure gradient signal generated by the laminar and turbulent are the input to the neuromast models, and the output is neuromast displacement in both the time and frequency domain. For the turbulent flows, a second series of plots show high pass filtered $(>10 \mathrm{~Hz})$ results. In essence, this looks at the higher frequency motions of the neuromast. The corner frequency of $10 \mathrm{~Hz}$ would be at the far extreme of the fish filtering abilities, and should not be considered realistic. Experimental evidence of Chagnaud et al. (2008) suggests that the corner frequency might be around 3-5 Hz, which is still much higher than suggested by equation 5.3.

\subsubsection{Laminar flow results}

Figures 5-12 - 5-14 present the results for laminar flow past a sculpin body sitting on the floor. In plot $(\mathrm{A})$ of each figure, the rms velocity fluctuations are shown around the sculpin. The data is sampled at $200 \mathrm{~Hz}$ for $2 \mathrm{~s}$, at locations $1.1 \mathrm{~cm}$ above the floor, which is at the level of the mid plane of the sculpin. Data points are shown for 
an area $20 \mathrm{x} 12 \mathrm{~cm}$, with 9 points every square $\mathrm{cm}$. For $2 \mathrm{~cm} / \mathrm{s}$ flow, the mean $\mathrm{rms}$ velocity fluctuations over the area shown is about $8 \mu \mathrm{m} / \mathrm{s}$, increasing to $80 \mu \mathrm{m} / \mathrm{s}$ for $4 \mathrm{~cm} / \mathrm{s}$ flow, and $200 \mu \mathrm{m} / \mathrm{s}$ for $8 \mathrm{~cm} / \mathrm{s}$ flow. Localized fluctuations behind the pectoral fins increase from less than $0.5 \mathrm{~mm} / \mathrm{s}$ to over $1.5 \mathrm{~mm} / \mathrm{s}$ as the flow speed increases. The location of the sphere is not seen in the plot because it is located 1.6 $\mathrm{cm}$ above the floor, the flow alterations from its presence can be seen. It's location relative to the fish is identified in plot (D) of each figure.

In plot (B) of each figure, the pressure gradient signal at each location on the source side lateral line is shown. This includes the infraorbital, supraorbital, mandibular, and trunk canal lateral line locations. Fluctuations due to turbulence are evident at many locations. These fluctuations result from the turbulence that develops in the floor boundary layer, and also along the boundary layer that develops along the side of the fish. At locations where the turbulence is reduced, $50 \mathrm{~Hz}$ signal oscillations are visible at repeating intervals of $500 \mathrm{~ms}$ on, $500 \mathrm{~ms}$ off patterns. The velocity amplitude is $1.2 \mathrm{~mm} / \mathrm{s}$, as in the still water case, for flow speeds of $2-4 \mathrm{~cm} / \mathrm{s}$. At 8 $\mathrm{cm} / \mathrm{s}$ flow, the velocity amplitude was increased to $6.0 \mathrm{~mm} / \mathrm{s}$.

The signal $50 \mathrm{~Hz}$ oscillations are not easily seen as flow speeds increase for two reasons. The first is that they are tiny in comparison with large increases in the range of mean pressure gradient. At $2 \mathrm{~cm} / \mathrm{s}$ flow the values range from -10 to 15 $\mathrm{Pa} / \mathrm{m}$, for $4 \mathrm{~cm} / \mathrm{s}$ flow the values range from -50 to $100 \mathrm{~Pa} / \mathrm{m}$, and for $8 \mathrm{~cm} / \mathrm{s}$ flow the values range from -300 to $500 \mathrm{~Pa} / \mathrm{m}$. Even at closest neuromast location, the maximum pressure gradient induced by the dipole oscillations is about $0.1 \mathrm{~Pa} / \mathrm{m}$ for the $1.2 \mathrm{~mm} / \mathrm{s}$ velocity amplitude signal, and about $0.5 \mathrm{~Pa} / \mathrm{m}$ for the $6.0 \mathrm{~mm} / \mathrm{s}$ velocity amplitude signal. The second reason is the turbulent fluctuations are much larger than this at most neuromast locations.

Plot (C) of each figure shows the neuromast displacement at one lateral line location, which is identified in plot (D) of each figure. It turns out that this particular neuromast location, positioned above the pectoral fin insertion point, has relatively low noise attributes at many flow speeds. This allows the filtering properties of the canal neuromast system to make the $50 \mathrm{~Hz}$ signal visible both in the temporal domain 
and also in the spectral domain, as shown in plot (E) of each figure. The displacement for the three types of neuromast model - spherical, flat plate, and cylindrical - are compared in plots $(\mathrm{C})$ and $(\mathrm{E})$. Their responses to mean signals show that the flat plate model has an almost 1:1 scaling, while the cylindrical model is always lessoned and the spherical model is greatly increased (about 6:1 scaling). This is in contrast to the spectral results, which shows an overlap of the spherical and cylindrical models at the low frequencies $(<10 \mathrm{~Hz})$, an overlap of the spherical and flat plate models from about 20-60 Hz, and overlap of all three above $100 \mathrm{~Hz}$.

Figure 5-15 shows the emergence of a dominant spectral peak at $50 \mathrm{~Hz}$ over four laminar flow simulations at $8 \mathrm{~cm} / \mathrm{s}$. Whereas the $1.2 \mathrm{~mm} / \mathrm{s}$ velocity amplitude of the oscillating sphere was sufficient to be seen above the noise at the neuromast location above the pectoral fin, this was not the case for the $8 \mathrm{~cm} / \mathrm{s}$ flow. In fact, it only becomes visible around the $4 \mathrm{~mm} / \mathrm{s}$ velocity amplitude range, which can be seen in the spectral plot where the velocity amplitude was set to $4.3 \mathrm{~mm} / \mathrm{s}$. At this amplitude, the signal is greater than the noise for that one selected neuromast. There were a number of locations needing less stimulation than this $(2-3 \mathrm{~mm} / \mathrm{s})$, but most needed this and much more.

An open question is how a fish might handle neuromast locations which have good signal-to-noise ratios, but for which the signal stimulus is relatively small next to other noisy neighboring neuromast locations. This is avenue for future research. At least for the laminar flow situations, the $50 \mathrm{~Hz}$ signals that appear in the spectral domain are also visible in the time domain, allowing for the possibility of phase-locking to take place.

\subsubsection{Turbulent flow results}

Most naturally occurring flows have turbulence associated with them. The ocean has turbulent motions in the bottom boundary layers and in the near surface mixing region (Grant and Madsen, 1986), and small creeks can have velocity fluctuations as high as $70 \mathrm{~mm} / \mathrm{s}$ for 10-25 cm/s flow speeds (Hanke, 2001). In the lab experiments of Chagnaud et al. (2008), a flow which had an upstream and downstream collimator 
$2 \mathrm{~cm} / \mathrm{s}$ laminar flow characteristics
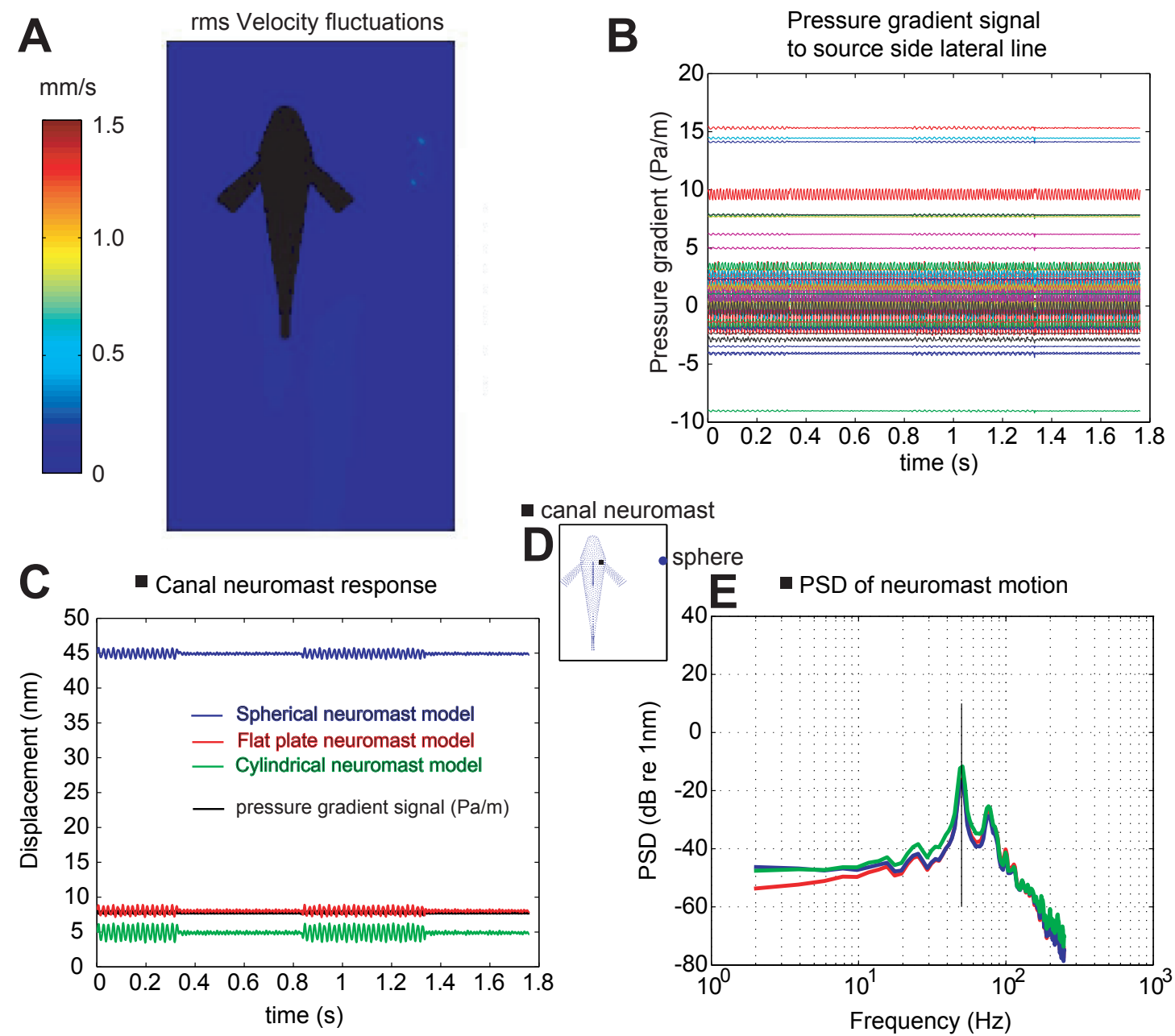

Figure 5-12: $2 \mathrm{~cm} / \mathrm{s}$ laminar flow characteristics. (A) rms velocity fluctuations, sampled at $200 \mathrm{~Hz}$ for $2 \mathrm{~s}$, at locations $1.1 \mathrm{~cm}$ above the floor. Data points are shown for an area 20x12 cm, with 9 points every square $\mathrm{cm}$. (B) Pressure gradient signal at each location along the source side lateral line (infraorbital, supraorbital, mandibular, and trunk). Two types of fluctuations can be seen: turbulence generated fluctuations, and also $50 \mathrm{~Hz}$ vibrations for $500 \mathrm{~ms}$ on, $500 \mathrm{~ms}$ off patterns. The velocity amplitude of the $3 \mathrm{~mm}$ radius sphere is $1.2 \mathrm{~mm} / \mathrm{s}$. (C) Spherical, flat plat, and cylindrical model neuromast displacement at one lateral line location, as shown in (D). (E) The power spectral density of the neuromast displacement at that one location. At this location, the $50 \mathrm{~Hz}$ signal is evident both in the temporal domain and in the spectral domain as the dominant peak. 
$4 \mathrm{~cm} / \mathrm{s}$ laminar flow characteristics
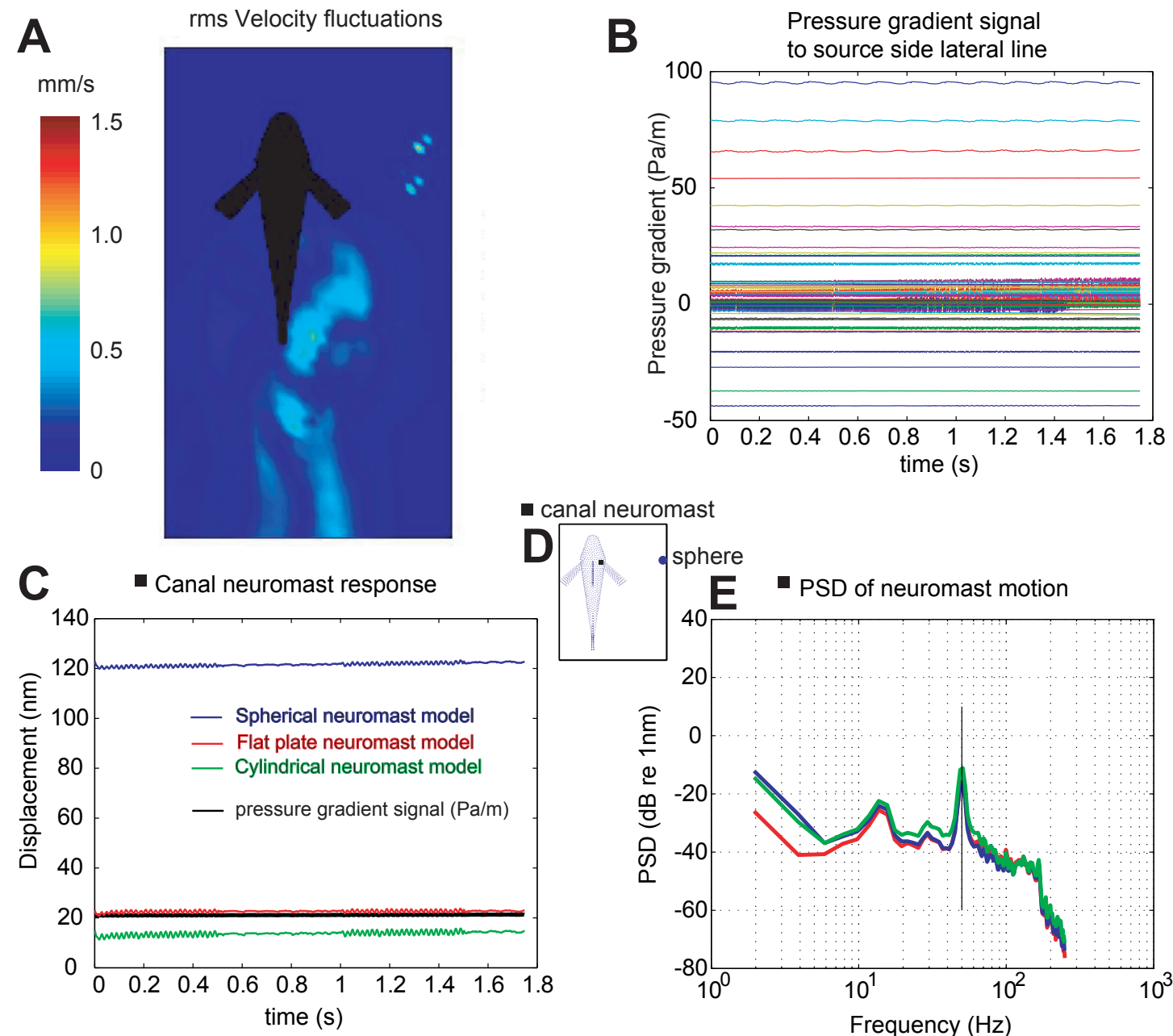

canal neuromast

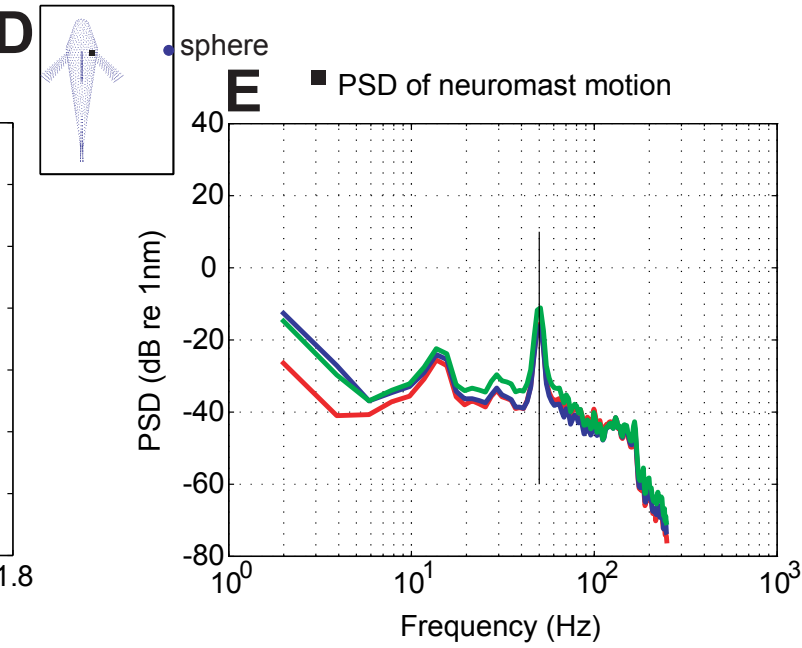

Figure 5-13: $4 \mathrm{~cm} / \mathrm{s}$ laminar flow characteristics. (A) rms velocity fluctuations, sampled at $200 \mathrm{~Hz}$ for $2 \mathrm{~s}$, at locations $1.1 \mathrm{~cm}$ above the floor. Data points are shown for an area 20x12 cm, with 9 points every square $\mathrm{cm}$. Turbulence generated by the pectoral fins extension into the oncoming flow is visible. (B) Pressure gradient signal at each location along the source side lateral line (infraorbital, supraorbital, mandibular, and trunk). Turbulent fluctuations can be seen, but the $50 \mathrm{~Hz}$ vibrations for 500 $\mathrm{ms}$ on, $500 \mathrm{~ms}$ off patterns are not easily distinguished. The velocity amplitude of the $3 \mathrm{~mm}$ radius sphere is $1.2 \mathrm{~mm} / \mathrm{s}$. (C) Spherical, flat plat, and cylindrical model neuromast displacement at one lateral line location, as shown in (D). (E) The power spectral density of the neuromast displacement at that one location. At this location, the noise is small enough that the $50 \mathrm{~Hz}$ signal is evident both in the temporal domain and in the spectral domain as the dominant peak. There is more energy in lower frequencies than for the $2 \mathrm{~cm} / \mathrm{s}$ laminar flow. 
$8 \mathrm{~cm} / \mathrm{s}$ laminar flow characterisitics
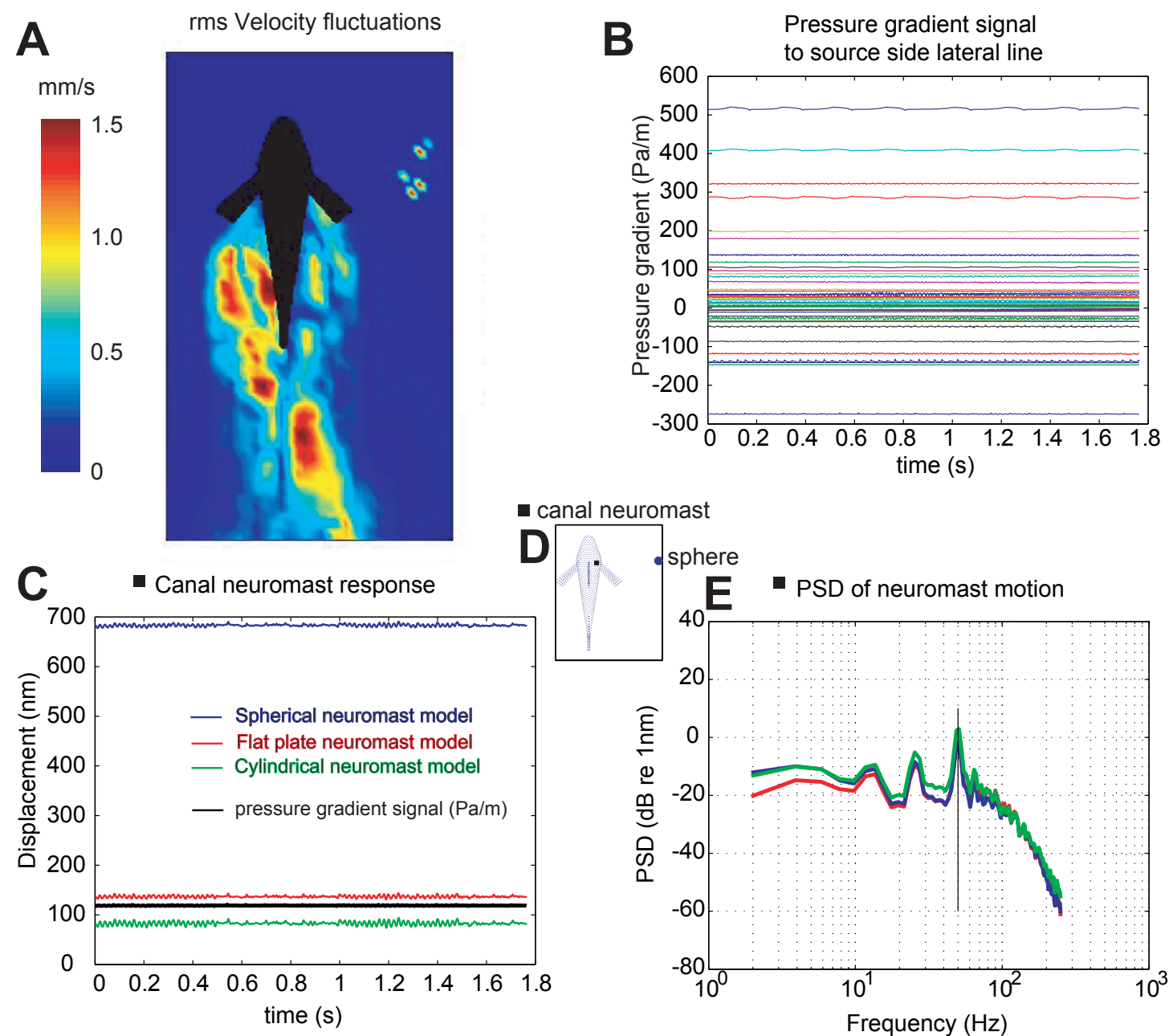

Figure 5-14: $8 \mathrm{~cm} / \mathrm{s}$ laminar flow characteristics. (A) rms velocity fluctuations, sampled at $200 \mathrm{~Hz}$ for $2 \mathrm{~s}$, at locations $1.1 \mathrm{~cm}$ above the floor. Data points are shown for an area 20x12 cm, with 9 points every square $\mathrm{cm}$. Turbulence generated by the pectoral fins extension into the oncoming flow is visible on both sides of the fish. (B) Pressure gradient signal at each location along the source side lateral line (infraorbital, supraorbital, mandibular, and trunk). Turbulent fluctuations can be seen, but the $50 \mathrm{~Hz}$ vibrations for $500 \mathrm{~ms}$ on, $500 \mathrm{~ms}$ off patterns are not visible. The velocity amplitude of the $3 \mathrm{~mm}$ radius sphere is $6.0 \mathrm{~mm} / \mathrm{s}$. (C) Spherical, flat plat, and cylindrical model neuromast displacement at one lateral line location, as shown in (D). (E) The power spectral density of the neuromast displacement at that one location. The velocity amplitude was raised to $6.0 \mathrm{~mm} / \mathrm{s}$ so that the $50 \mathrm{~Hz}$ peak would rise above the low frequency noise, which is significantly more than that found at 2 and $4 \mathrm{~cm} / \mathrm{s}$ flows. 
Detection threshold: emergence of the dominant signal peak for $8 \mathrm{~cm} / \mathrm{s}$ laminar flow
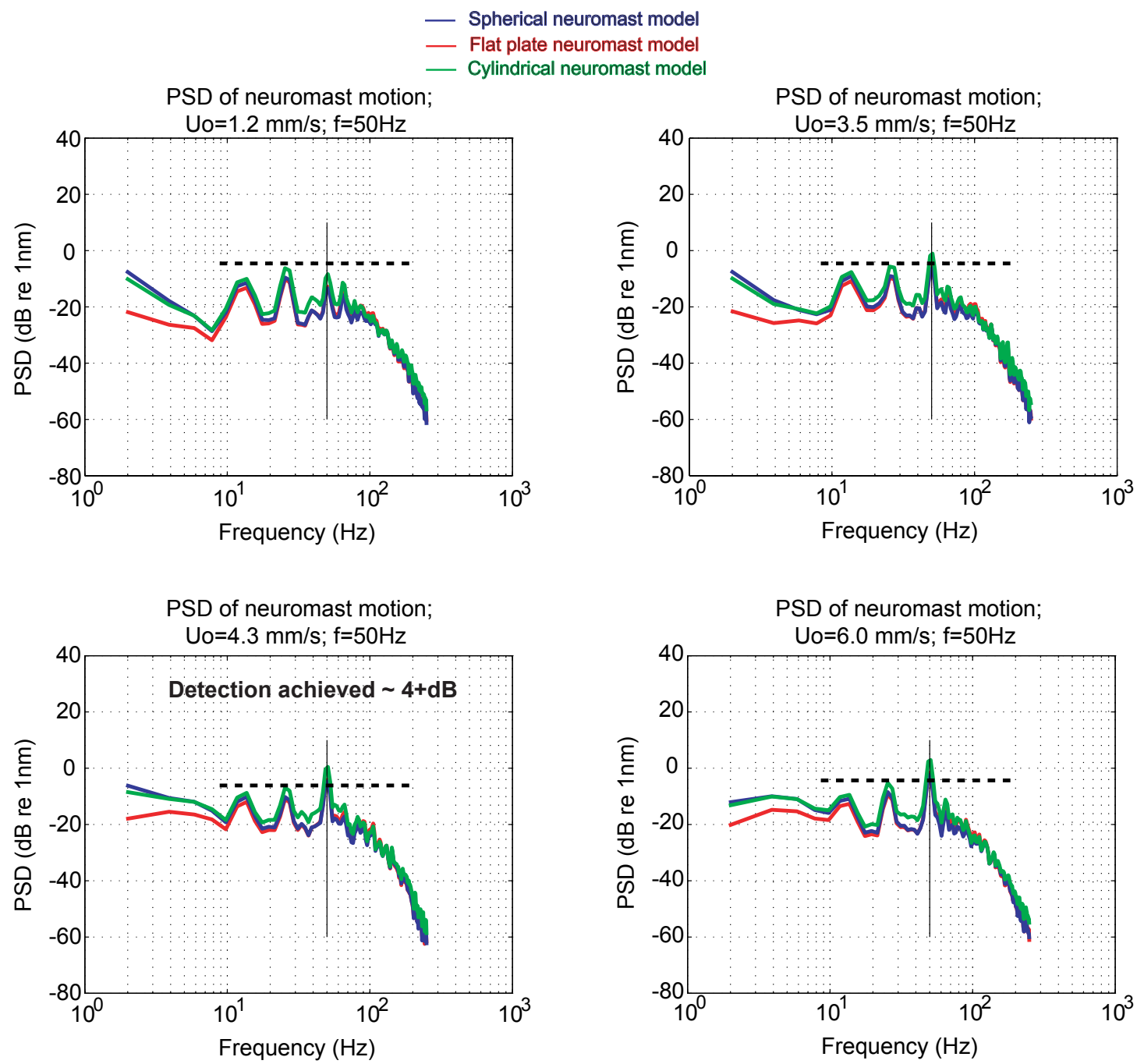

Figure 5-15: Emergence of the dominant, prey induced, spectral peak in $8 \mathrm{~cm} / \mathrm{s}$ laminar flow. Four separate flow simulations were run, each with increasing velocity amplitude. The sphere location and neuromast location is the same as shown in the previous figures. 
produced velocity fluctuations which increased linearly with flow speed. The turbulence intensity, expressed as the ratio of the root mean square velocity fluctuations to the mean velocity, can be estimated to be about $10 \%$ for their data, leading to rms velocity fluctuions as high as $13 \mathrm{~mm} / \mathrm{s}$ for $13 \mathrm{~cm} / \mathrm{s}$ flow speeds.

For the turbulent simulations shown in figures 5-16 - 5-27, the rms velocity fluctuations ranged from $0.7-4 \mathrm{~mm} / \mathrm{s}$. As described previously, 9 points per square $\mathrm{cm}$, located $1.1 \mathrm{~cm}$ above the bottom, where used to sample velocity at $200 \mathrm{~Hz}$ for $2 \mathrm{~s}$. The mean turbulent value was calculated for the whole region, the $20 \mathrm{x} 12 \mathrm{~cm}$ area (excluding the fish location), and that value used to characterize the level of turbulence. As individual plots show, local velocity fluctuations varied significantly, have much higher values than the mean, while other areas had smaller fluctuations. Pockets of turbulence would conduct downstream with the flow, so that the $2 \mathrm{~s}$ window is just a brief snapshot of local conditions. Note also the color scale for the $2 \mathrm{~cm} / \mathrm{s}$ and 4 $\mathrm{cm} / \mathrm{s}$ flow with rms velocity $\sim 0.7-1 \mathrm{~mm} / \mathrm{s}$ (figures $5-16$ and $5-20$ ) ranges from $0-1.5$ $\mathrm{mm} / \mathrm{s}$, like the laminar flow plots, but ranges from $0-5 \mathrm{~mm} / \mathrm{s}$ for all other turbulence plots.

The figures are arranged in pairs, with the first showing the turbulent flow field, the pressure gradient signal to the lateral line, the resulting neuromast motions, and the spectrum of these motions. The second figure in each pair shows how the hair cell would filter those motions (with a time constant of $10 \mathrm{~ms}$ ). The units are labeled as neural equivalent displacements, meaning that these are the motions that would correspond to internal currents and potentials produced by the hair cell. Likewise, the filtered spectrum corresponds to what would produced by the hair cell.

The second figure in each pair of figures also presents the high pass $(>10 \mathrm{~Hz})$ filtered motions. Neither the neuromast motions nor hair cell filtering can produce results like this, only being able to filter low frequencies motions up to possibly 5 Hz. However, they are real oscillations and motions in the sense that these are the tiny fluctuations present in the signal, superposed on the lower frequency signals. They are instructive in providing temporal results of what can be seen in the spectral results, namely the dominance or masking of the $50 \mathrm{~Hz}$ source signal in relation to 
other high frequency signals.

In the $2 \mathrm{~cm} / \mathrm{s}$ flow with $\mathrm{rms}$ velocity fluctuations of $0.7 \mathrm{~mm} / \mathrm{s}$, the pressure gradient signal to the lateral line has doubled from that of laminar flow (compare figures 5-16B and $5-16 \mathrm{~B})$. This is due to the nature of the turbulence generation in these simulations, which essentially randomly injects particles upstream, causing pressure oscillations which result in flow field fluctuations. Plot (C) shows the canal neuromast motions resulting from it pressure gradient signal, with the three types of neuromast models compared. Two of the models show that some of the excursions would reach saturation if not further filtered by the hair cell. Tiny fluctuations are visible, corresponding to the dominant spectral peak at $50 \mathrm{~Hz}$ seen in plot (E).

The second figure in this pair (figure 5-17) shows the work of the hair cell to reduce the large neuromast excursions previously seen, to motions equivalent to having $20 \mathrm{~nm}$ excursions. This is a result of the gating apparatus and hair cell chemistry adapting to the neuromast motions. Plot (B) shows the equivalent spectral representation has reduced the low frequency components, and lowered the noise level overall. The oscillations which were hardly visible in figure 5-16C are now comparably larger to the reduced low frequency signal components. Plots $(\mathrm{C})$ and $(\mathrm{E})$ show how the oscillations due to the source dipole signal are much larger than the noise present. Even though there are noise excursions which are larger, they are infrequent in time and density, unlike the source signal. In the temporal sense, the constancy of the source oscillation amplitude and the density of energy expressed are like a ringing bell against the relatively static background.

The situation changes with an increased level of turbulence. The $3.5 \mathrm{~mm} / \mathrm{s}$ velocity amplitude of the source signal now becomes swamped by equally loud noise. This is evident in the neuromast motion response spectrum see in figure $5-18 \mathrm{E}$, where the $50 \mathrm{~Hz}$ peak is no larger than any of the nearby peaks, and orders of magnitude smaller than the low frequency peaks. The neuromast motions are have $300 \mathrm{~nm}$ excursions, following the dominant low frequency signal. The hair cell filtering (figure 5-19A) brings these excursions well within the saturation limit, but static noise is not reduced enough for the source signal to be seen (plot B). This is confirmed in the high 
frequency neuromast motions (plots $\mathrm{C}$ and $\mathrm{E}$ ), which shows that the noise works to distort the $50 \mathrm{~Hz}$ oscillations in a way that makes them unrecognizable.

The $4 \mathrm{~cm} / \mathrm{s}$ flow with $\sim 1 \mathrm{~mm} / \mathrm{s}$ rms velocity fluctuations (figure 5-20) shows patchy regions of turbulence. The neuromast motions (plot C) are similar to those seen in the $2 \mathrm{~cm} / \mathrm{s}$ flow with $\sim 0.7 \mathrm{~mm} / \mathrm{s} \mathrm{rms}$ velocity fluctuations (figure $5-16$ ), as are the spectral results. In fact, hair cell filtering and high pass filtering show almost identical results with source oscillations that stand out against pockets of lesser background noise (compare figures 5-21 and 5-17).

Increasing the turbulence fluctuations to $3 \mathrm{~mm} / \mathrm{s}$ results in increased neuromast excursions and spectral masking, similar to what was seen in the $2 \mathrm{~cm} / \mathrm{s}$ flow with $2 \mathrm{~mm} / \mathrm{s}$ turbulent fluctuations (compare figures 5-22 and 5-18). However, the noisy fluctuations are intermittent enough that although the $50 \mathrm{~Hz}$ source signal is distorted by the noise, the high pass filtered signal shows traces of signal in its constancy and density (see figure 5-23E).

This constancy of amplitude and density of signal energy in time are more clearly evident for the $8 \mathrm{~cm} / \mathrm{s}$ flow that has turbulent fluctuations of $\sim 2 \mathrm{~mm} / \mathrm{s}$, in between the $2 \mathrm{~cm} / \mathrm{s}$ and $4 \mathrm{~cm} / \mathrm{s}$ flow with $1 \mathrm{~mm} / \mathrm{s}$ and $3 \mathrm{~mm} / \mathrm{s}$ turbulent fluctuations (figure $5-25 \mathrm{C}, \mathrm{E})$. It should be noted that the source amplitude has increased its velocity amplitude to $6.0 \mathrm{~mm} / \mathrm{s}$, as compared to $3.5 \mathrm{~mm} / \mathrm{s}$ for the $2 \mathrm{~cm} / \mathrm{s}$ turbulent flows and 4.3 $\mathrm{mm} / \mathrm{s}$ for the $4 \mathrm{~cm} / \mathrm{s}$ turbulent flows. The source oscillations are made distinguishable against the background noise because of the hair cell filtering, which lowers the low frequency energy enough for a spectral peak to emerge (compare figures 5-24E and 5-25B). The remarkable design of the hair cell in using the gating apparatus tension to dictate the signal rather than the neuromast position is seen in the reduction of neuromast motions, from the deflections of $1000-3500 \mathrm{~nm}$ (figure 5-24C), to the equivalently tensioned motions of $50 \mathrm{~nm}$ excursions (figure 5-25A).

Increasing the turbulent fluctuations to $4 \mathrm{~mm} / \mathrm{s}$ is too much for the $6.0 \mathrm{~mm} / \mathrm{s}$ source signal to be detected, and figures 5-26 and 5-27 show similar masking and disappearance of the signal for the $8 \mathrm{~cm} / \mathrm{s}$ flow as seen in the $2 \mathrm{~cm} / \mathrm{s}$ with lesser turbulence and a lesser source amplitude (compare with figures 5-18 and 5-19). 
$2 \mathrm{~cm} / \mathrm{s}$ flow characteristics with turbulence; $\mathrm{rms}$ vel $0.7 \mathrm{~mm} / \mathrm{s}$
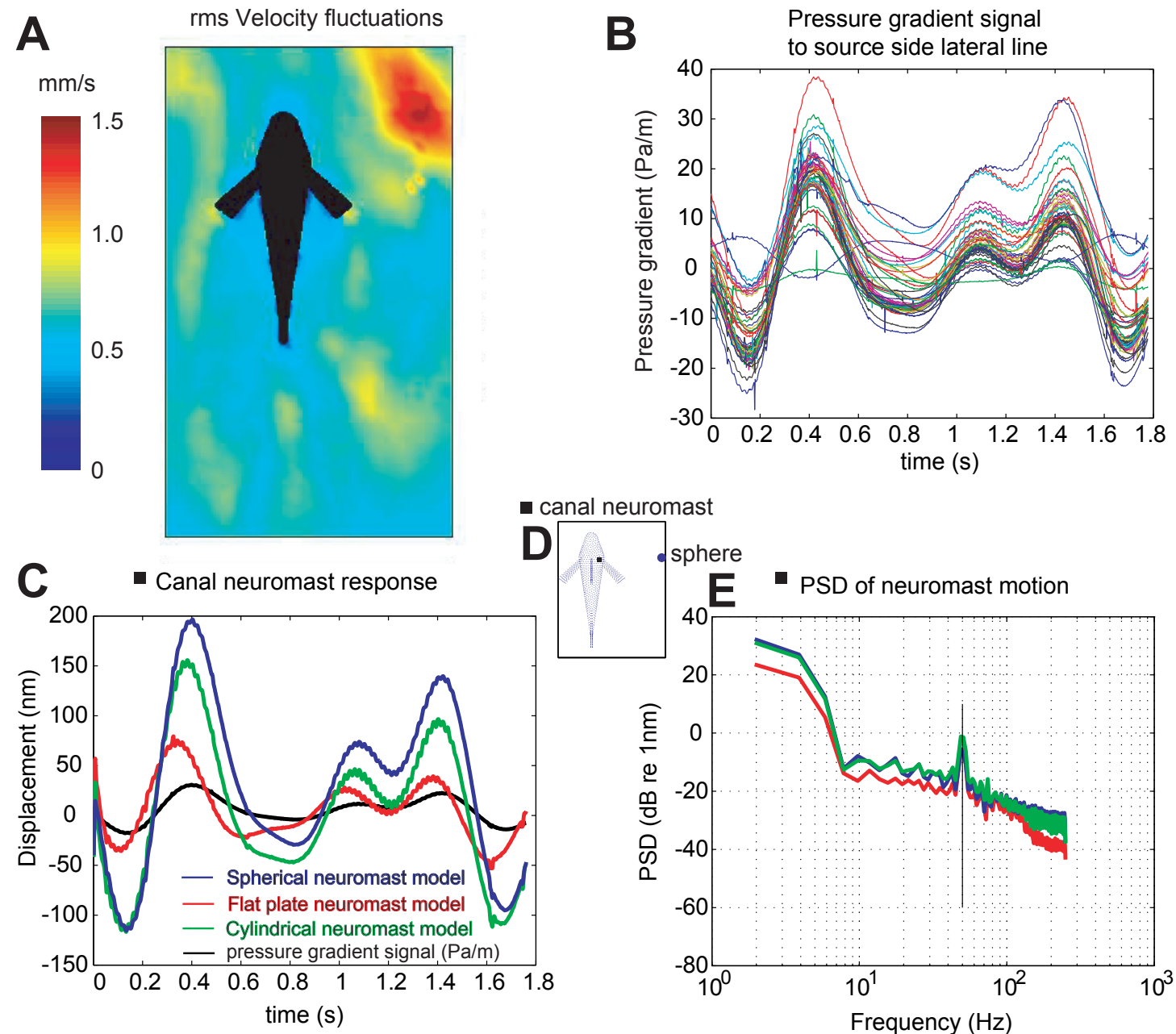

Figure 5-16: $2 \mathrm{~cm} / \mathrm{s}$ turbulent flow. (A) Root mean square velocity fluctuations, sampled at $200 \mathrm{~Hz}$ for $2 \mathrm{~s}$, at locations $1.1 \mathrm{~cm}$ above the floor. Data points are shown for an area $20 \times 12 \mathrm{~cm}$, with 9 points every square $\mathrm{cm}$. The mean rms velocity fluctuation for the region is $0.7 \mathrm{~mm} / \mathrm{s}$. (B) Pressure gradient signal at each location along the source side lateral line (infraorbital, supraorbital, mandibular, and trunk), over $1.8 \mathrm{~s}$ of the $2 \mathrm{~s}$ window. (C) Spherical, flat plat, and cylindrical model neuromast displacement at one lateral line location, responding to its pressure gradient signal. (D) The location of the selected canal neuromast, relative to the sphere. The sphere oscillates at $50 \mathrm{~Hz}$, for $500 \mathrm{~ms}$ on and $500 \mathrm{~ms}$ off repeating patterns. The velocity amplitude is $3.5 \mathrm{~mm} / \mathrm{s}$, and the sphere radius is $3 \mathrm{~mm}$. (E) The power spectral density of the neuromast displacement, sampled at $500 \mathrm{~Hz}$, and calculated using Welch's method (1967). 
Hair cell response and high pass response to $2 \mathrm{~cm} / \mathrm{s}$ flow wih turbulence; rms vel $\sim 0.7 \mathrm{~mm} / \mathrm{s}$
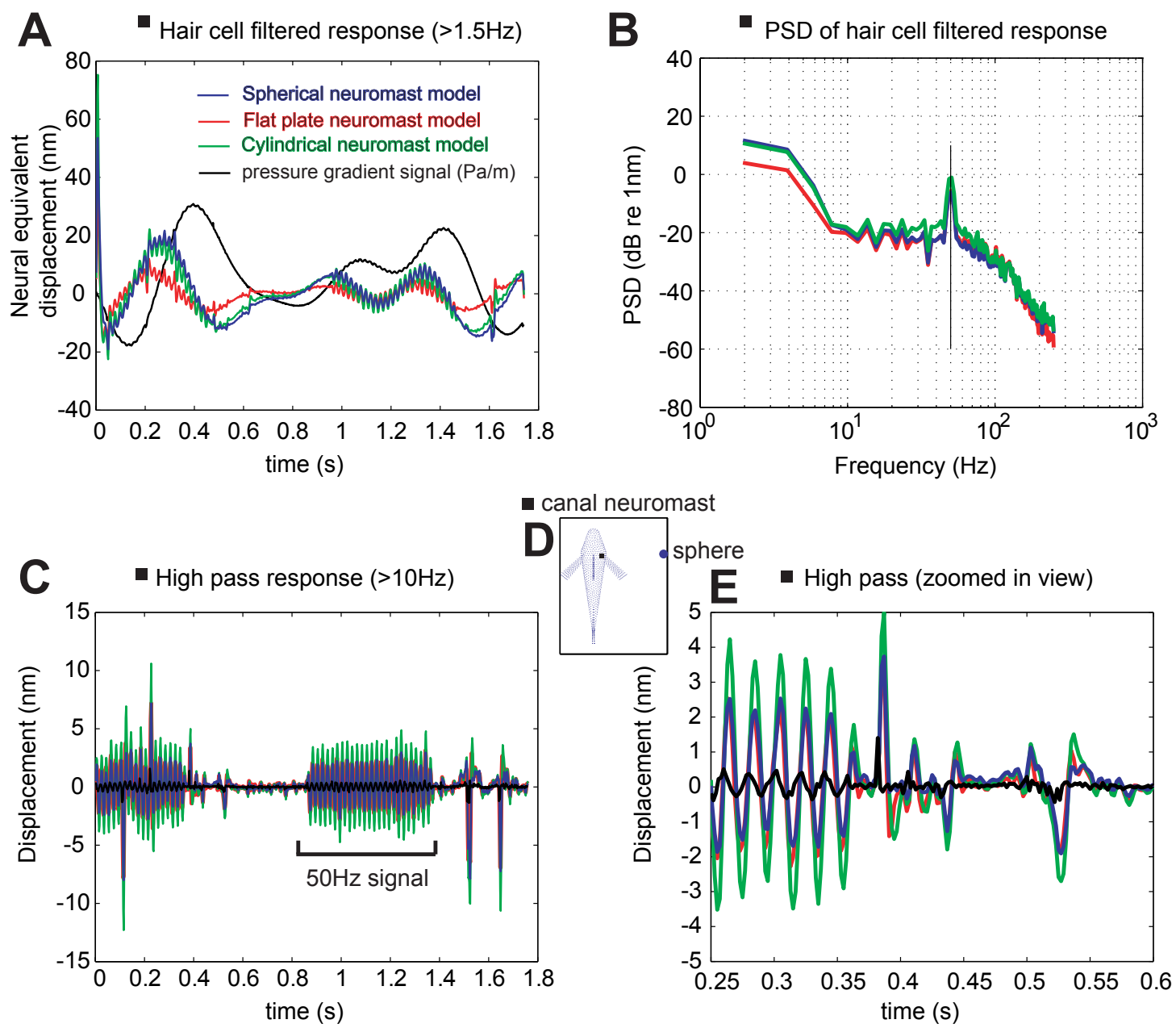

Figure 5-17: Hair cell filtering and high pass $(>10 \mathrm{~Hz})$ response to the $2 \mathrm{~cm} / \mathrm{s}$ flow with $0.7 \mathrm{~mm} / \mathrm{s}$ turbulent fluctuations. (A) The hair cell filters the neuromast displacement through its gating apparatus and internal chemistry. The neural equivalent displacements are those motions which correlate to the currents and potentials of the hair cell. (B) The power spectral density of the hair cell output, in relation to the neural equivalent motions. (C) The high frequency neuromast motions associated with its pressure gradient signal. This is not the fish's filtering, but it is what the fish is experiencing within the filtered frequency range. (D) Canal neuromast location relative to the fish body and vibrating sphere. (E) A zoomed in view of part of the signal in (C). Sampling of signals and sphere parameters given in figure 5-16. 
$2 \mathrm{~cm} / \mathrm{s}$ flow characteristics with turbulence; $\mathrm{rms}$ vel $2 \mathrm{~mm} / \mathrm{s}$
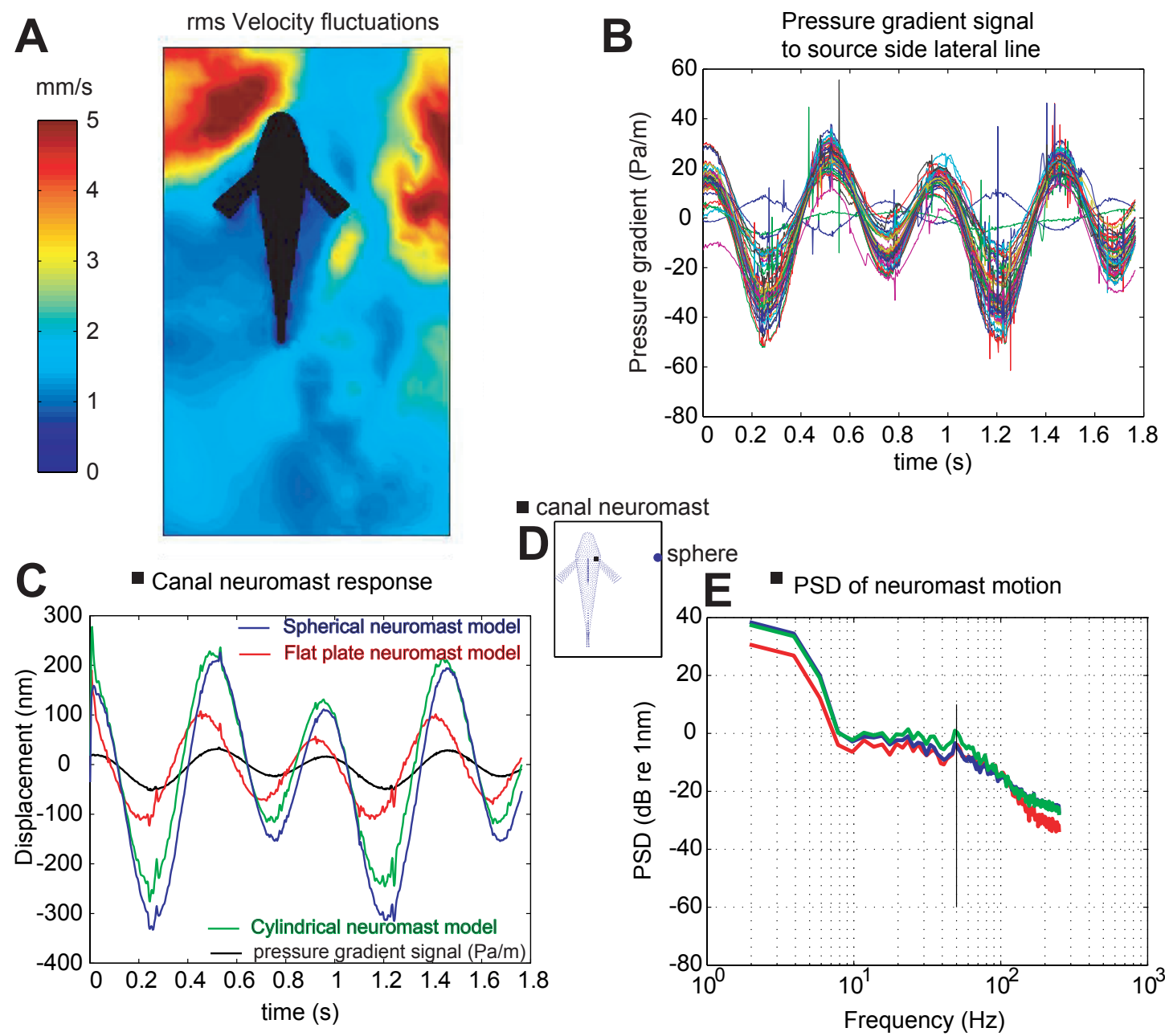

Figure 5-18: $2 \mathrm{~cm} / \mathrm{s}$ flow with $2 \mathrm{~mm} / \mathrm{s}$ mean rms velocity fluctuations. Explanation and description as in figure 5-16. Parameters that differ: color scale is from 0-5 mm/s. 
Hair cell response and high pass response to $2 \mathrm{~cm} / \mathrm{s}$ flow wih turbulence; rms vel $\sim 2 \mathrm{~mm} / \mathrm{s}$
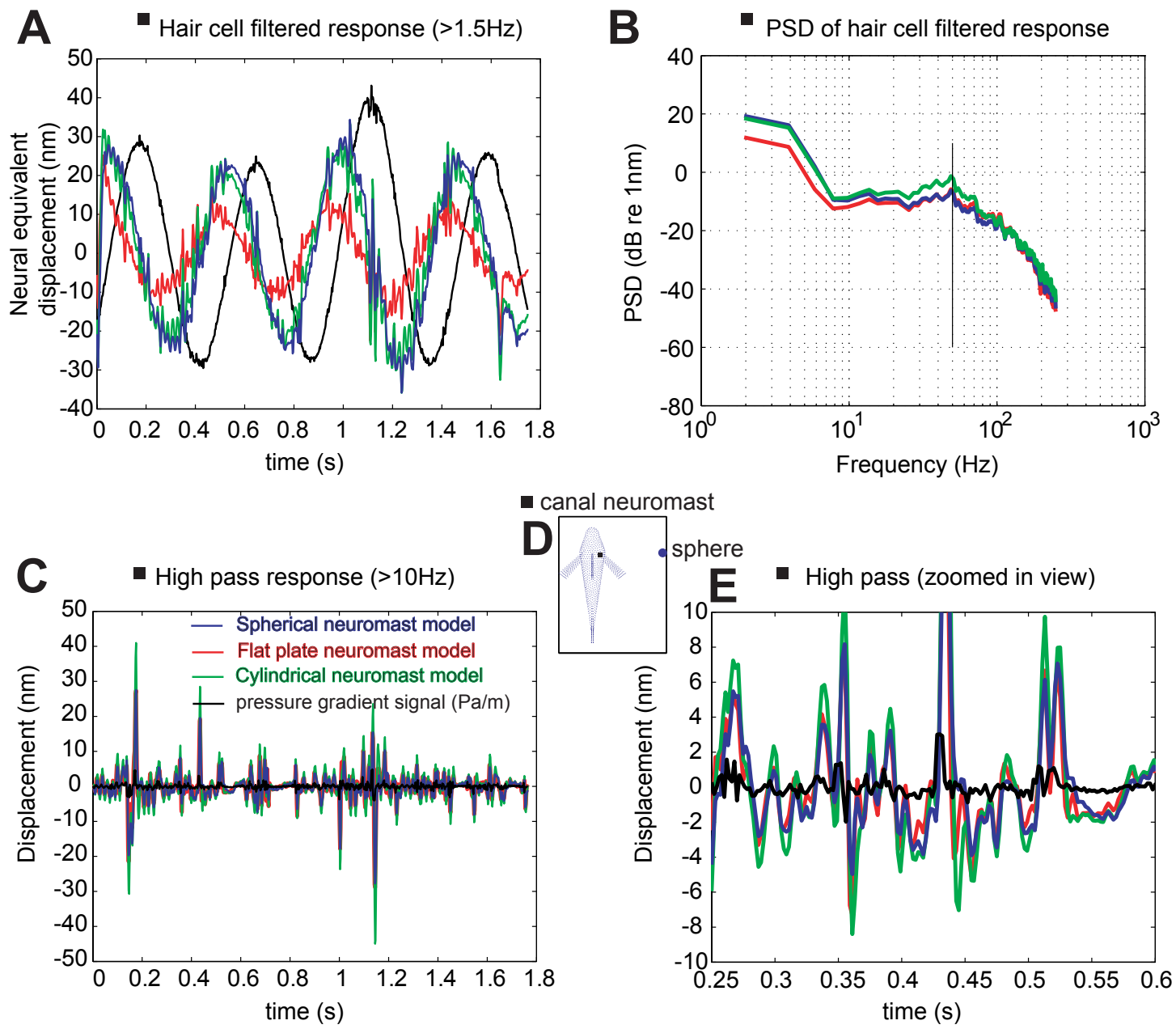

Figure 5-19: Hair cell filtering of the $2 \mathrm{~cm} / \mathrm{s}$ flow with $2 \mathrm{~mm} / \mathrm{s}$ mean $\mathrm{rms}$ velocity fluctuations. Explanation and description as in figure 5-17. 
$4 \mathrm{~cm} / \mathrm{s}$ flow characteristics with turbulence; $\mathrm{rms}$ vel $\sim 1 \mathrm{~mm} / \mathrm{s}$
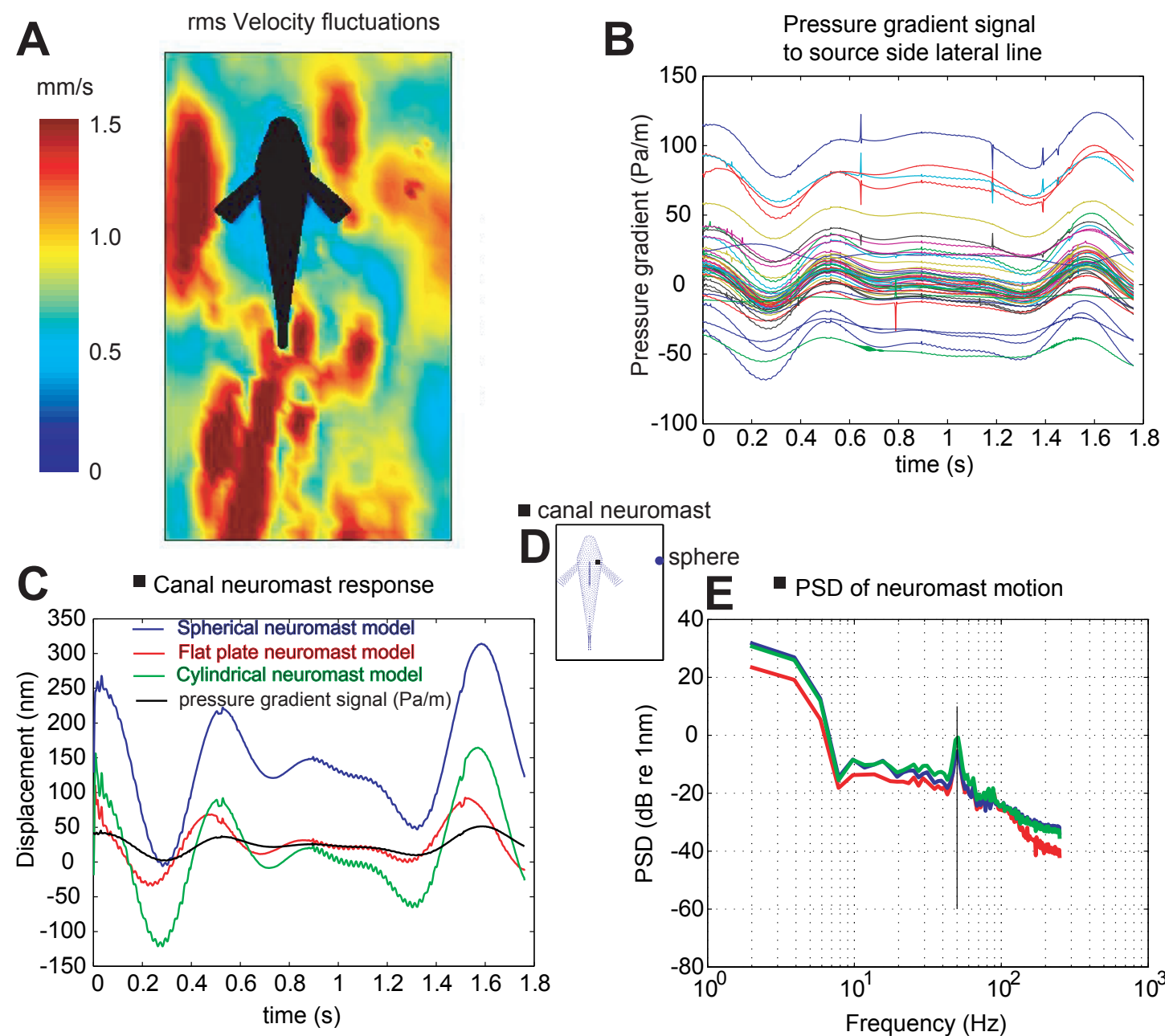

- canal neuromast

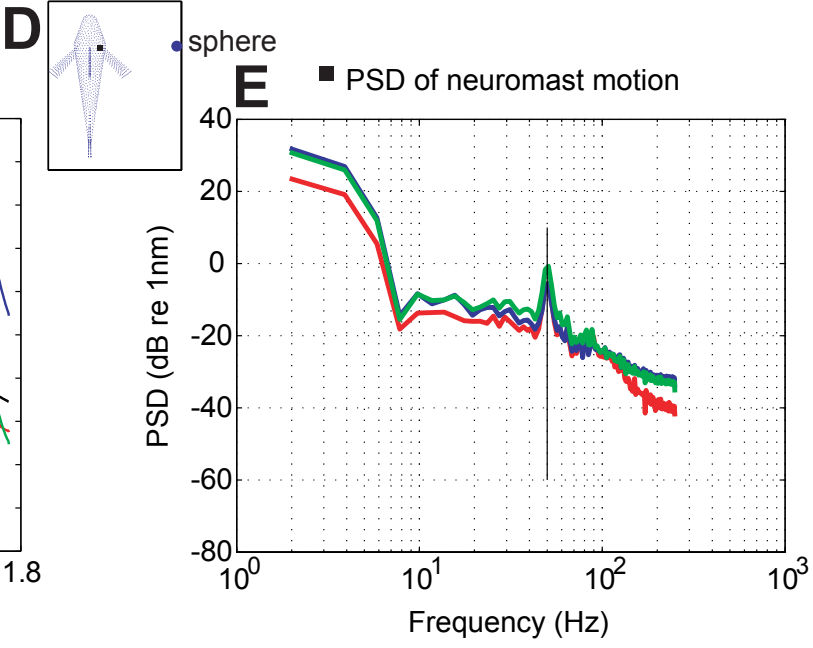

Figure 5-20: $4 \mathrm{~cm} / \mathrm{s}$ flow with $1 \mathrm{~mm} / \mathrm{s}$ mean rms velocity fluctuations. Explanation and description as in figure 5-16. Parameters that differ: sphere velocity amplitude is $4.3 \mathrm{~mm} / \mathrm{s}$. 
Hair cell response and high pass response to $4 \mathrm{~cm} / \mathrm{s}$ flow wih turbulence; rms vel $\sim 1 \mathrm{~mm} / \mathrm{s}$
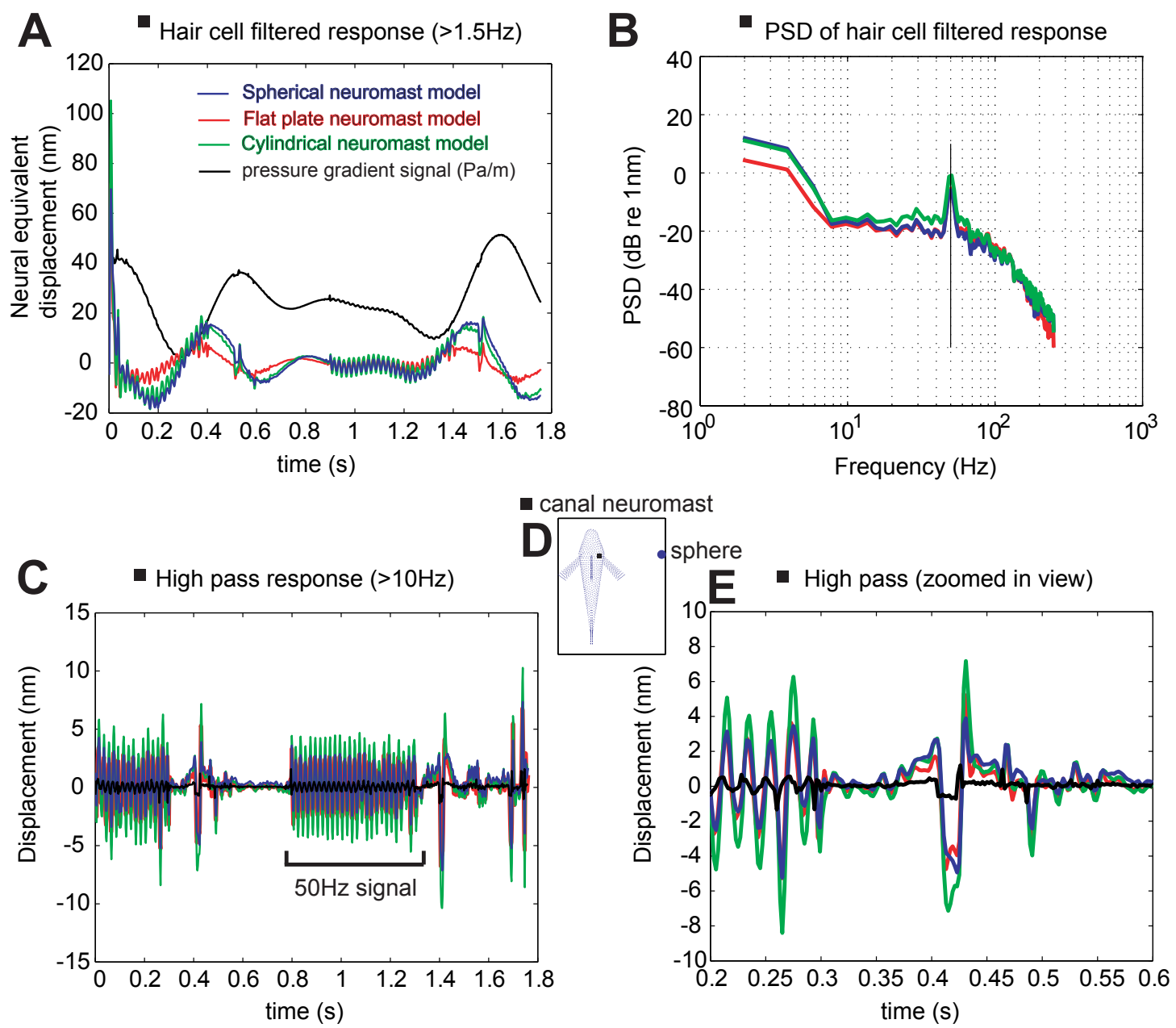

Figure 5-21: Hair cell filtering of the $4 \mathrm{~cm} / \mathrm{s}$ flow with $1 \mathrm{~mm} / \mathrm{s}$ mean $\mathrm{rms}$ velocity fluctuations. Explanation and description as in figure 5-17. Parameters that differ: sphere velocity amplitude is $4.3 \mathrm{~mm} / \mathrm{s}$. 
$4 \mathrm{~cm} / \mathrm{s}$ flow characteristics with turbulence; $\mathrm{rms}$ vel $\sim 3 \mathrm{~mm} / \mathrm{s}$
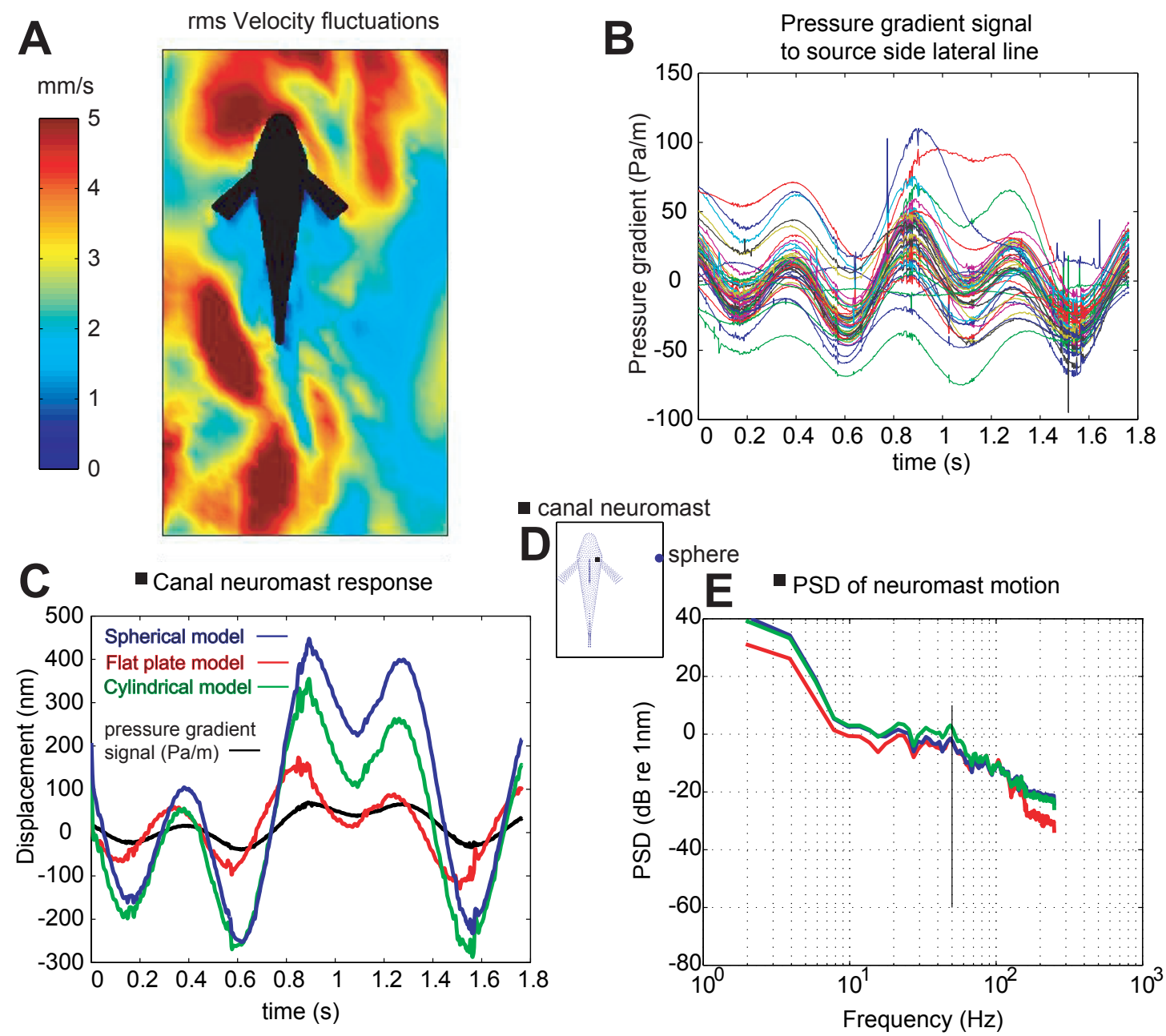

Figure 5-22: $4 \mathrm{~cm} / \mathrm{s}$ flow with $3 \mathrm{~mm} / \mathrm{s}$ mean rms velocity fluctuations. Explanation and description as in figure 5-16. Parameters that differ: color bar range is from 0-5 $\mathrm{mm} / \mathrm{s}$; sphere velocity amplitude is $4.3 \mathrm{~mm} / \mathrm{s}$. 
Hair cell response and high pass response to $4 \mathrm{~cm} / \mathrm{s}$ flow wih turbulence; rms vel $\sim 3 \mathrm{~mm} / \mathrm{s}$
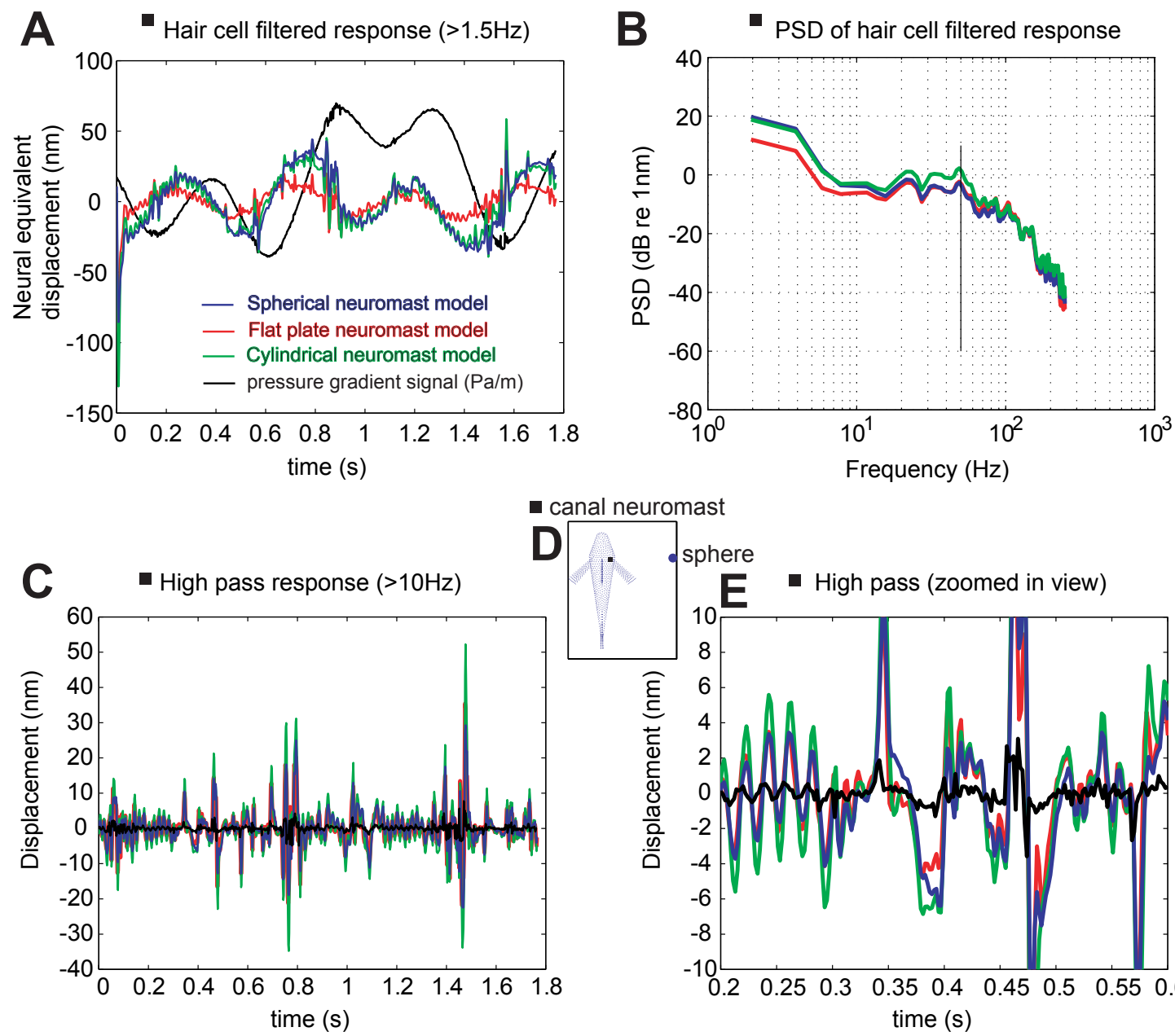

canal neuromast

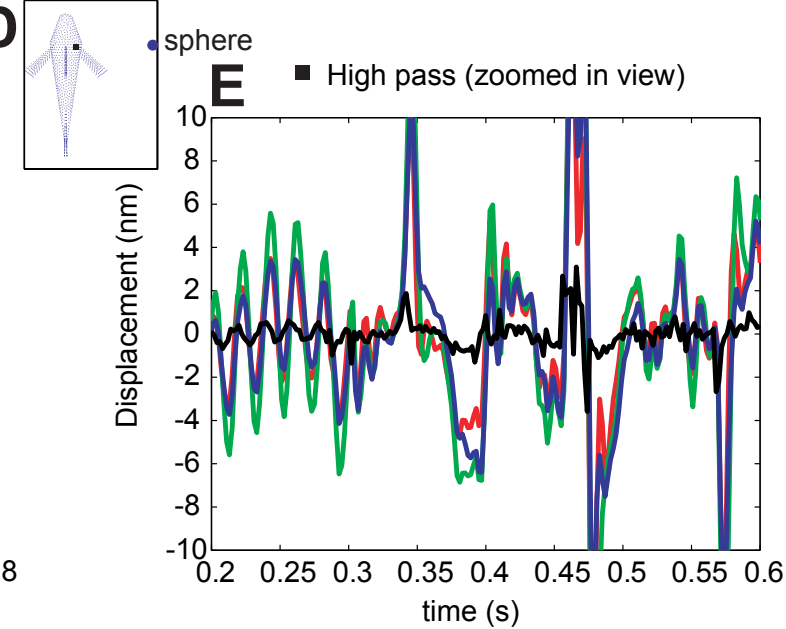

Figure 5-23: Hair cell filtering of the $4 \mathrm{~cm} / \mathrm{s}$ flow with $3 \mathrm{~mm} / \mathrm{s}$ mean $\mathrm{rms}$ velocity fluctuations. Explanation and description as in figure 5-17. Parameters that differ: sphere velocity amplitude is $4.3 \mathrm{~mm} / \mathrm{s}$. 
$8 \mathrm{~cm} / \mathrm{s}$ flow characteristics with turbulence; $\mathrm{rms}$ vel $2 \mathrm{~mm} / \mathrm{s}$
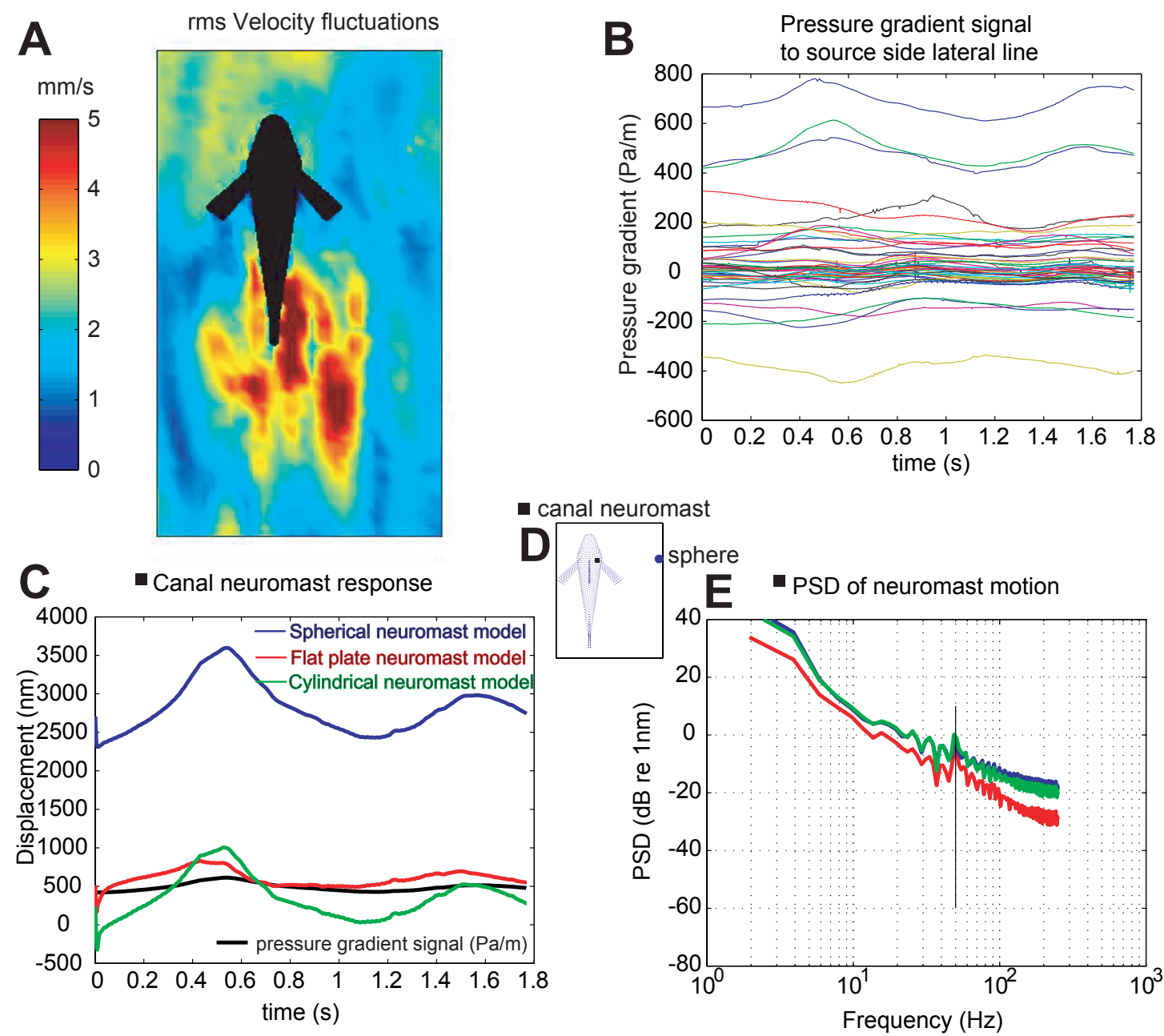

Figure 5-24: $8 \mathrm{~cm} / \mathrm{s}$ flow with $2 \mathrm{~mm} / \mathrm{s}$ mean $\mathrm{rms}$ velocity fluctuations. Explanation and description as in figure 5-16. Parameters that differ: color bar range is from 0-5 $\mathrm{mm} / \mathrm{s}$; sphere velocity amplitude is $6.0 \mathrm{~mm} / \mathrm{s}$. 
Hair cell response and high pass response to $8 \mathrm{~cm} / \mathrm{s}$ flow wih turbulence; rms vel $\sim 2 \mathrm{~mm} / \mathrm{s}$
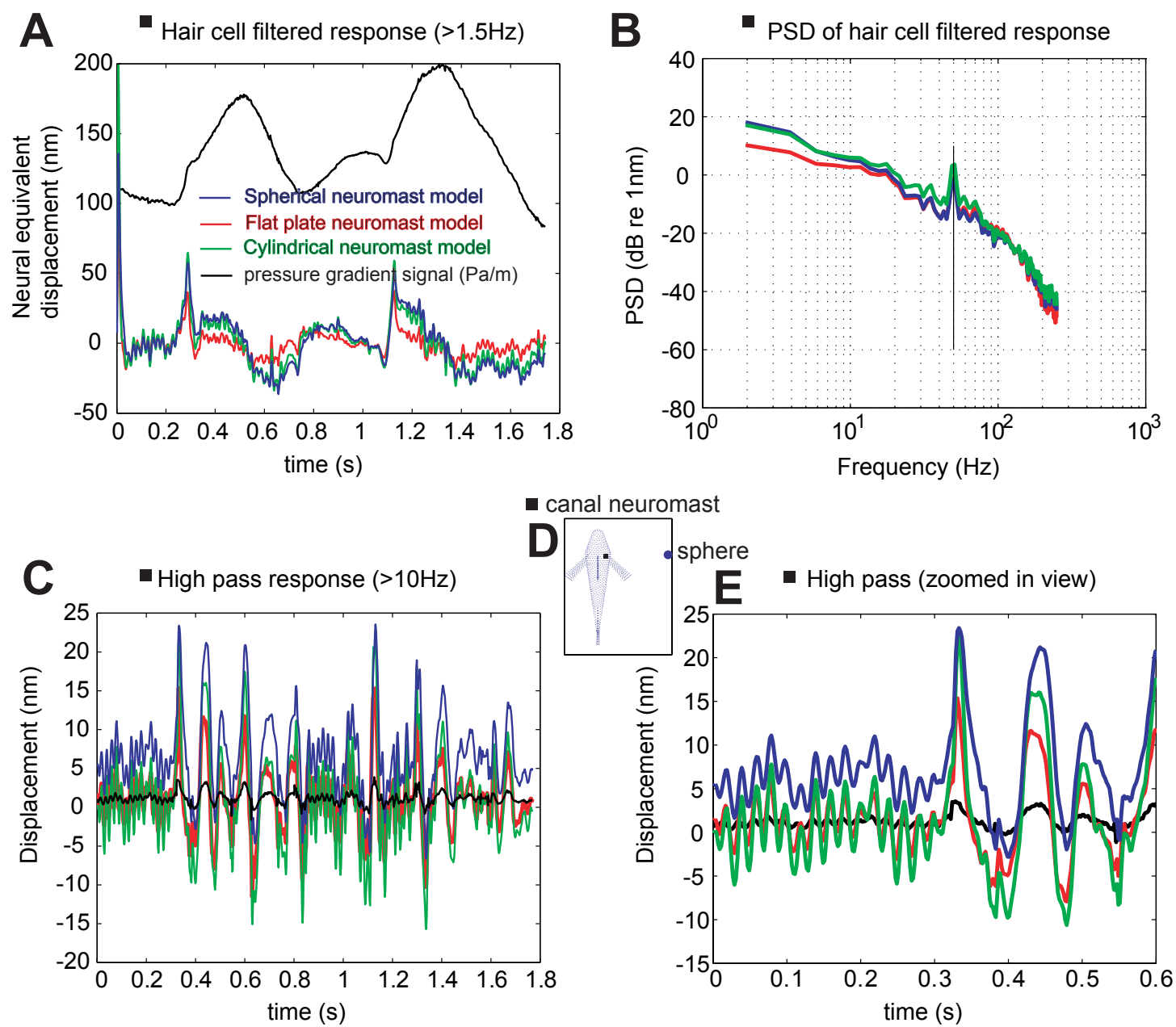

Figure 5-25: Hair cell filtering of the $8 \mathrm{~cm} / \mathrm{s}$ flow with $2 \mathrm{~mm} / \mathrm{s}$ mean $\mathrm{rms}$ velocity fluctuations. Explanation and description as in figure 5-17. Parameters that differ: sphere velocity amplitude is $6.0 \mathrm{~mm} / \mathrm{s}$. 
$8 \mathrm{~cm} / \mathrm{s}$ flow characteristics with turbulence; $\mathrm{rms}$ vel $\sim 4 \mathrm{~mm} / \mathrm{s}$
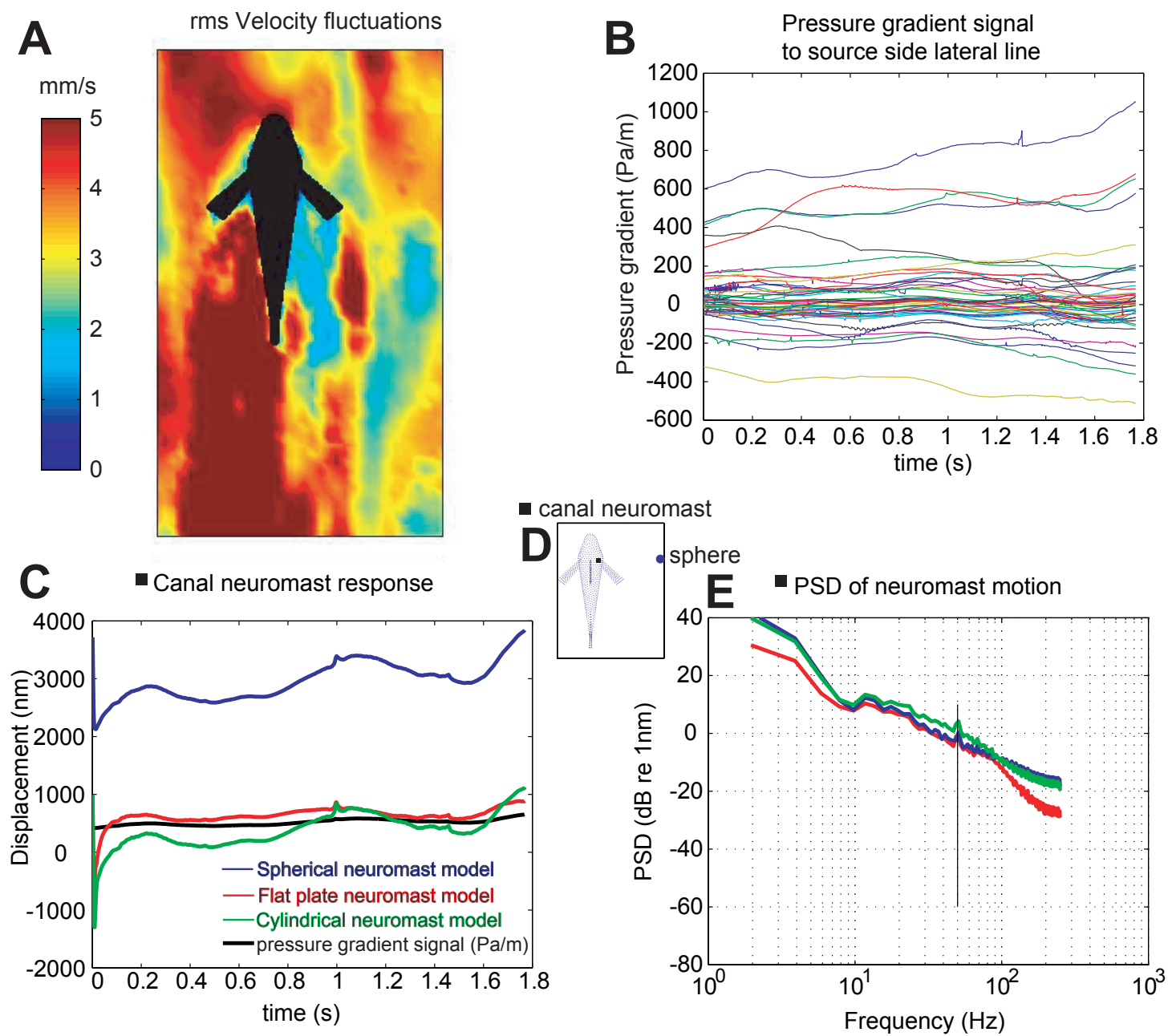

Figure 5-26: $8 \mathrm{~cm} / \mathrm{s}$ flow with $4 \mathrm{~mm} / \mathrm{s}$ mean $\mathrm{rms}$ velocity fluctuations. Explanation and description as in figure 5-16. Parameters that differ: color bar range is from 0-5 $\mathrm{mm} / \mathrm{s}$; sphere velocity amplitude is $6.0 \mathrm{~mm} / \mathrm{s}$. 
Hair cell response and high pass response to $8 \mathrm{~cm} / \mathrm{s}$ flow wih turbulence; rms vel $\sim 4 \mathrm{~mm} / \mathrm{s}$
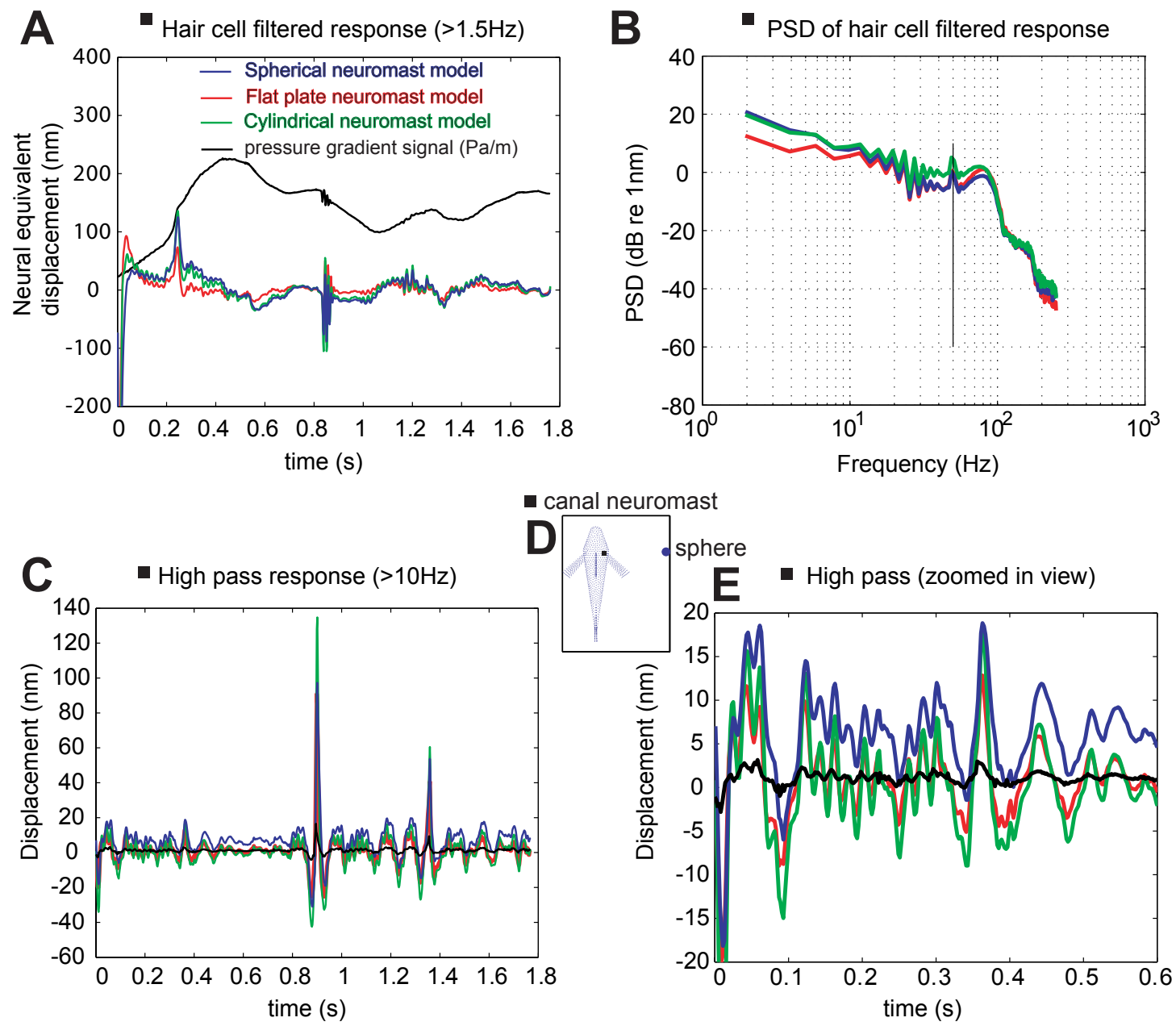

Figure 5-27: Hair cell filtering of the $8 \mathrm{~cm} / \mathrm{s}$ flow with $4 \mathrm{~mm} / \mathrm{s}$ mean $\mathrm{rms}$ velocity fluctuations. Explanation and description as in figure 5-17. Parameters that differ: sphere velocity amplitude is $6.0 \mathrm{~mm} / \mathrm{s}$. 


\subsubsection{Effect of extended fins on signal detection}

One question to address is the possibility that the reduced region of flow behind the fins leads to improved signal detection. Figure 5-28 shows the sculpin fish with fins retracted. The $8 \mathrm{~cm} / \mathrm{s}$ background flows have similar levels of turbulence to the flows being compared with the sculpin having fins extended (see figures 5-24 and 5-26).

Figure 5-2 showed that a boundary layer of reduced flow speed develops down the side of a sculpin shaped body in oncoming flow. When turbulence is introduced, figure 5-24 shows at a certain point down the side of the body the surface boundary layer flow becomes turbulent. In figure 5-26, an increased level of turbulence in the flow triggers the turbulence in the surface boundary layer at a point farther upstream on the fish body. For the sculpin canal lateral line, the physical separation of canal pore openings, at an approximate distance of $2 \mathrm{~mm}$, act as natural spatial filters. The high frequency fluctuations that develop because of turbulence in the boundary layer are essentially low pass filtered by the spatial integration from pore to pore.

To quantitatively compare differences, figure 5-29 presents the rms detrended pressure gradient fluctuations for all canal lateral line locations in the $8 \mathrm{~cm} / \mathrm{s}$ turbulent flows. In two of the flows the fins are retracted, and in two of the flows the fins are extended. The most notable difference is that the noise to the canal lateral line when fins are retracted is more even than when fins are extended. There is more noise near the tail of the sculpin when the fins are extended, as seen in neuromast numbers 50-60 which represent the trunk lateral line on the side of the fish opposite the oscillating sphere.

There does not appear to be a signal benefit to the lateral line in the region behind the pectoral fins. The recirculation and shedding around the pectoral fins and tail lead to increasing levels of noise. Since the lateral line goes up and around the pectoral fins, any signal detection benefits that might have been realized behind the fins, such as along the mid plane of the body, seem to be forfeited.

In all four flows, the level of noise to the canal lateral line fluctuates around the10 $\mathrm{Pa} / \mathrm{m}$ level. The fact that there is not a significant increase in noise, even when 
turbulence level increases, can be attributed to the way it was calculated. The step of detrending the pressure gradient signal, over the short time intervals of 400-500 ms, can have the effect of acting like a high pass filter, similar to what the hair cell filtering system might do. In the case of these particular flows, the increase in energy mostly occurs in the low frequencies $(<3 \mathrm{~Hz})$. The step of detrending has the possibility of reducing some of that energy.

\section{$8 \mathrm{~cm} / \mathrm{s}$ turbulent flow for a sculpin with fins retracted}
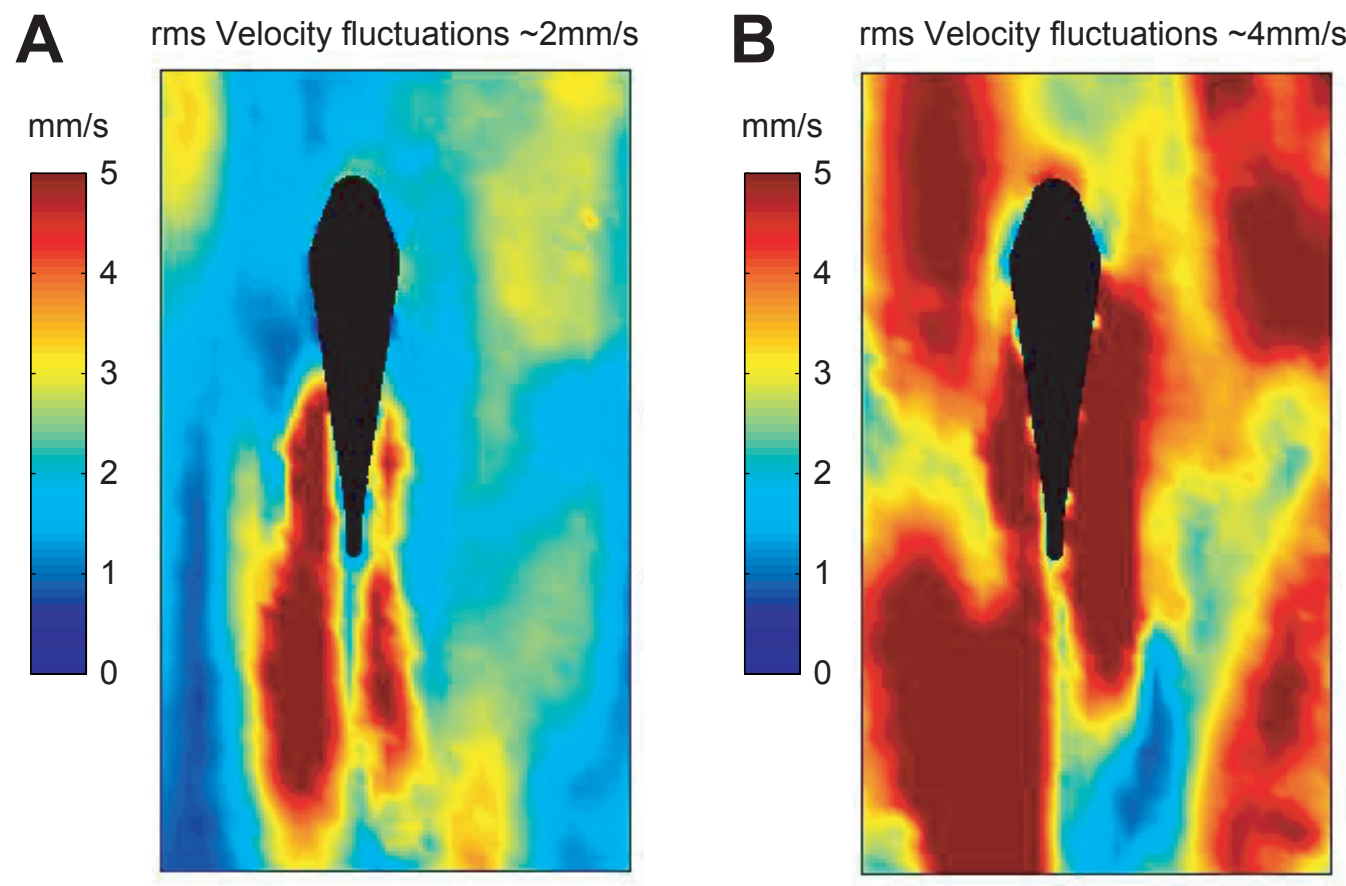

Figure 5-28: Two different turbulent flows are presented, with increasing levels of turbulence. Note that part way down the fish, the surface boundary layer becomes turbulent. See figure 5-2 to see the surface boundary layer. 


\section{SNR benefit with extended fins? Root mean square pressure gradient signal to the sculpin canal lateral line in $8 \mathrm{~cm} / \mathrm{s}$ turbulent flow}

\section{fins extended}

fins retracted
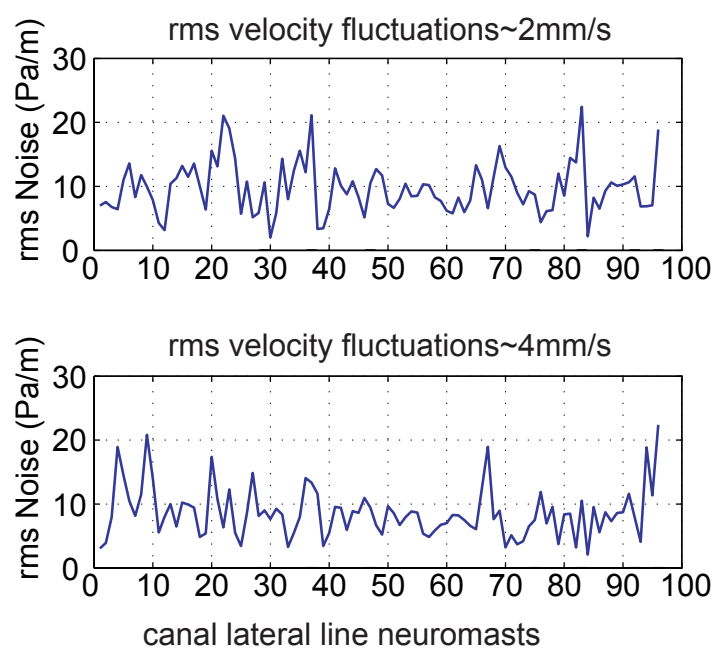
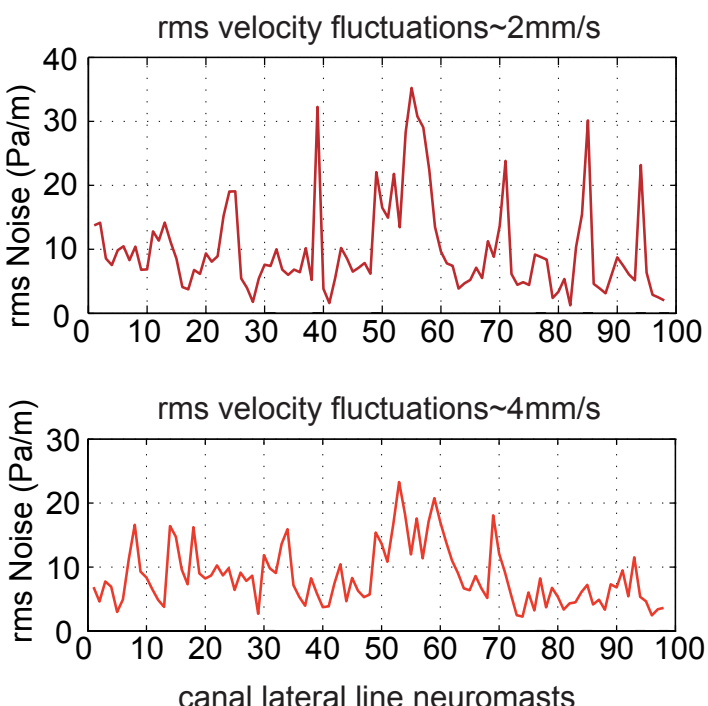

Figure 5-29: A quantitative comparison of the rms detrended pressure gradient signal to the canal lateral line for the situations when the fins are retracted vs. extended. Values are calculated over a period of 400-500 ms for each canal lateral line location on the body. The canal neuromast numbers correspond to the following locations with the following symbols: tr-trunk, io=infraorbital, $\mathrm{so}=$ supraorbital, $\mathrm{mn}=$ mandibular, oc-occiptal. Source side lateral line, fins retracted: $\operatorname{tr} \sim 1-22$; io $\sim 23-29$; so $\sim 30-37$; $\mathrm{mn} \sim 38-47$; Back side, fins retracted: $\operatorname{tr} \sim 48-68$; io $\sim 69-75$; so $\sim 76-83$; $\mathrm{mn} \sim$ 84-92; oc 93-96; Source side lateral line, fins extended: $\operatorname{tr} \sim 1-23$; io $\sim 24-31$; so $\sim$ 32-39; mn 40-49; Back side, fins extended: tr $\sim 50-70$; io $\sim 71-77$; so $\sim 78-85$; $\mathrm{mn}$ $\sim 86-94 ;$ oc $\sim 95-98$ 


\subsubsection{Threshold of detection in moving water}

The goal of this chapter is to understand how turbulent flows affect the signal detection abilities of fish, and of the sculpin in particular. The previous sections presented the building blocks that will enable this question to be addressed.

First, flows are characterized by their level of turbulence, based on the average of root mean square velocity fluctuations at many points around the fish. The sampling of points occurred at the mid plane level of the fish, so that sampling might reflect what the fish lateral line is being exposed to. Flow speed will be monitored, though from the start the premises of this chapter has been that it is the a.c. fluctuations that matter most.

Second, the hydrodynamic signal to the canal lateral line is characterized by the root mean square of the detrended pressure gradient fluctuations. Detrending represents a basic level of filtering, removing the mean and best linear fit from the selected segment. Since the oscillating sphere vibrates in recurring segments of $500 \mathrm{~ms}$ on, $500 \mathrm{~ms}$ off, the noise is measured during a 400-500 ms segment of the off cycle. That means two measurements are made during each characterization of the $2 \mathrm{~s}$ flow window. The measurements are not expected to be identical, since the flow can changed based on how the flow is evolving in response to turbulent structures present, and based on its interaction with the fish body. Error bars in the plots will represent this variability.

Signal detection thresholds can be calculated from these two parameters alone. The main theory being tested is the idea that detection occurs when the root mean square source signal fluctuations are greater than the background noise fluctuations. Using Hypothesis I, the noise is said to be set at a minimum threshold level, base-noise (still/moving), until it is surpassed by a.c. flow noise, which becomes the new threshold level. The minimum threshold level was determined from the experimental results of Kanter and Coombs (2003), using a vibrating sphere in still water. Figure 5-1 shows the root mean square pressure gradient signal to the canal lateral line (trunk and infraorbital), from a $3 \mathrm{~mm}$ sphere, oscillating at $50 \mathrm{~Hz}$, with 
a velocity amplitude of $1.2 \mathrm{~mm} / \mathrm{s}$, and located $5.5 \mathrm{~cm}$ from the longitudinal midline of the fish. It is an assumption of this thesis, that when the signal to at least three canal neuromasts is strong enough to overcome the inherent base-level noise, the fish responds with a strike. Deciding that three neuromasts need to be stimulated is not based on experimental evidence, and this will lead to some flexibility of the results presented. By choosing at least three neuromasts to be stimulated, some level of tolerance is built into this model to account for the fact that the $3 \mathrm{D}$ model is not the exact shape of the fish in the experiments. Using the results shown, and in consideration of other lateral line neuromasts (not shown), the base-noise in still water is set to $0.075 \mathrm{~Pa} / \mathrm{m}$.

Kanter and Coombs (2003) defined signal detection threshold as that level of sphere velocity amplitude which elicited a strike (or a motion to strike) from the sculpin. Note: their velocity amplitude is measured from peak to peak (crest to trough), whereas to be consistent with the notion of this thesis, the velocity amplitude is defined as half that value (base to crest or base to trough). Having used the same fish-sphere configuration, comparisons can be made with their results. Using equation 5.1, the sphere velocity amplitude which will elicit a response from the fish (hypothetically) can be calculated. Basically, the signal to the fish in still water is raised high enough to match the pressure gradient noise from the flow, and the velocity amplitude in still water $(1.2 \mathrm{~mm} / \mathrm{s})$ is multiplied by that amplification. The amount of amplification needed is neuromast specific, since each location will have a unique amount of noise, and also the level of the source signal strength is location specific.

Figure 5-30 presents the signal detection results for the same neuromast highlighted throughout the results (see plot E), the neuromast used to define the limit of detectability in still water stimulation. Plot (A) shows the velocity amplitudes needed to create the rms pressure gradient signal equal to the noise level characterized in the various flow simulations for that neuromast. There is the expected trend at each flow speed that as turbulence increases, the detection threshold level rises. Another expected trend is that the variability of detection threshold level also 


\section{Sphere velocity amplitude (Uo) needed for signal detection in background flow}
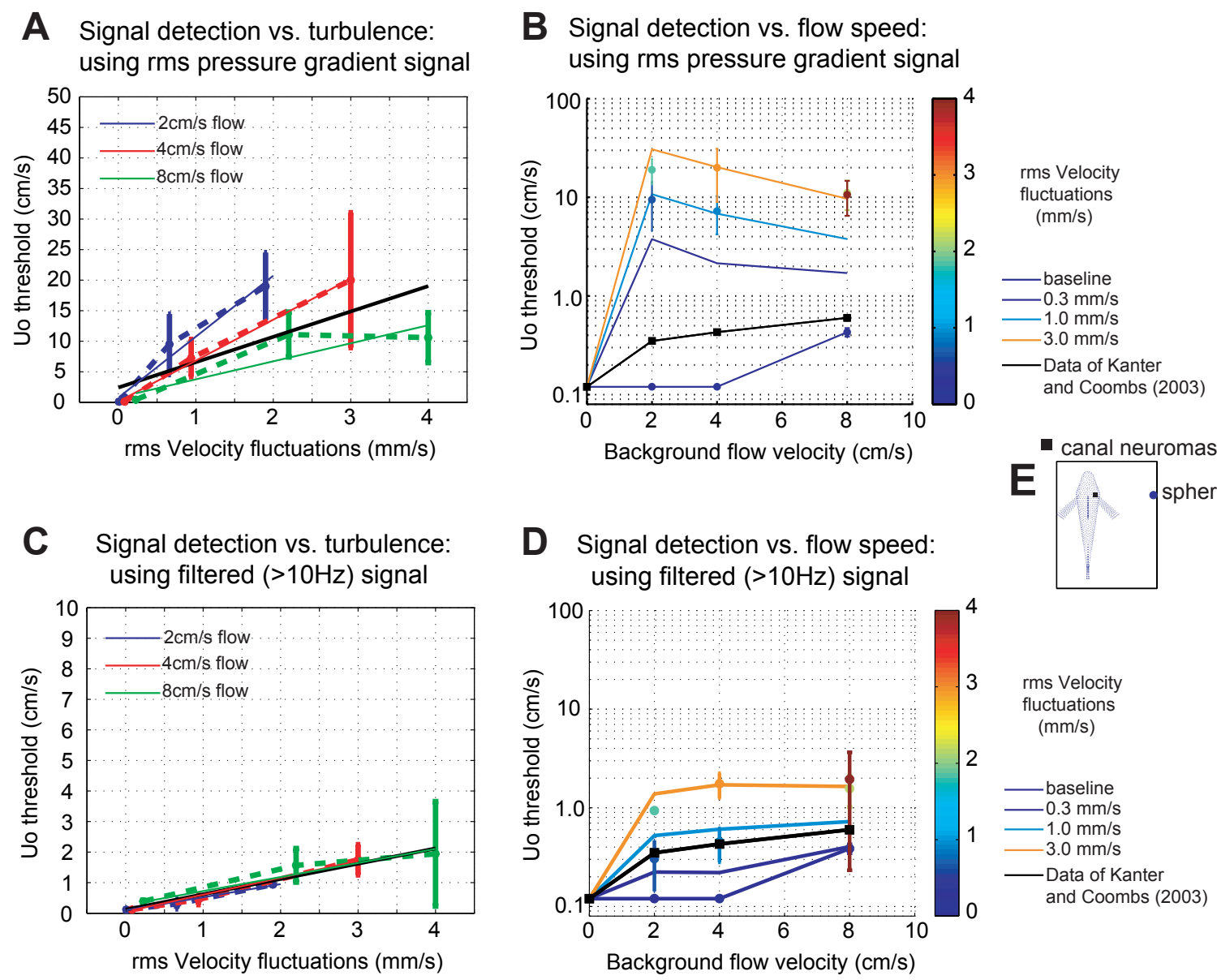

Figure 5-30: Detection thresholds based on pressure gradient signals to the canal line in $0-8 \mathrm{~cm} / \mathrm{s}$ background flows. The level of noise is determined by taking the root mean square of the detrended pressure gradient signal over two 400-500 ms intervals, and then averaging. The standard deviation of these two measurements is represented by the error bars. The turbulence is defined as the average of the root mean square velocity fluctuations at points $1.1 \mathrm{~cm}$ above the floor around the fish, over a $2 \mathrm{~s}$ interval. Threshold is calculated according to equation 5.1. (A) Detection threshold level vs. turbulence. Dotted lines connect data points. Solid lines are a best fit for each flow speed. (B) Detection threshold vs. flow speed. Isoturbulence contours are drawn using interpolated values from the best fit lines of (A). Baseline values of turbulence correspond to values obtained in the laminar flows. The data of Kanter and Coombs (2003) is plotted using the definition of velocity amplitude consistent with this thesis, defined as base-crest rather than peak-peak (crest to trough). The levels of turbulence depicted are hypothetical, and do not reflect the actual levels of turbulence present in their experiments (see text). (C) and (D) use the high pass filtered $(>10 \mathrm{~Hz})$ pressure gradient signal, rather than the detrended signal. All else is the same as in (A) and (B). (E) The location of the $3 \mathrm{~mm}$ sphere, relative to the fish and selected canal neuromast. base-noise is based on detection in still water, from $50 \mathrm{~Hz}$ oscillations and a velocity amplitude of $1.2 \mathrm{~mm} / \mathrm{s}$. 
increases with levels of turbulence, since more energetic flows have the possibility of being locally uneven and patchy. This characteristic will be flow dependent, since it matters greatly how the flow is generated. As shown in the previous section, these simulations have local and temporal variations in flow fluctuations.

There is the unexpected trend that at higher flow speeds, the threshold detection level decreases. Most likely this is a result of these patchy flows, where increasing flow speed enables the neuromast to sample more of the spatial flow field as it moves by. Patches of reduced turbulent energy will result in a lower mean value. This illustrates an important point: how the fish chooses to process the signals in its brain will make a difference as to what information it can extract. This point will be emphasized in plots $(\mathrm{C})$ and $(\mathrm{D})$.

Figure $5-30 \mathrm{~B}$ is a re-plot of the data in (A), but now threshold of detection is plotted against background flow speed in a manner similar to Kanter and Coombs (2003). Contours of isoturbulence are plotted using linear least squares fit to the data in (A) for each flow speed. The behaviorally determined responses of the sculpin are also plotted, but the level of turbulence for these data points is unknown. The plot shows that the Kanter and Coombs results are bounded flows containing a minimal amount of turbulence $(<0.3 \mathrm{~mm} / \mathrm{s})$. However, the fact that detection thresholds needed for small increases of turbulence are orders of magnitude higher than experimental results suggests that the threshold of detection as defined in this chapter is incorrect. This would also be contradicted by the experimental results of Engelmann et al. (2002) and Chagnaud et al. (2006) who found that increasing levels of flow speed resulted in minimal to no masking of the source signal for canal neuromasts.

Instead of defining the a.c. flow noise as the root mean square of the pressure gradient signal over all frequencies, the experimental evidence suggests an alternative. Define the a.c. flow noise over a high pass filtered range of frequencies. Since the integral of the power spectrum density (PSD) of the pressure gradient noise signal is equivalent to it's variance in the time domain, an integral over a band limited range of the PSD is equivalent to the variance of the signal in time containing those selected frequencies. Determining the correct range takes experimental verification, 
but choosing frequencies greater than $10 \mathrm{~Hz}$ should be a limit that fish will not be able to reach, setting a lower bound of sorts. It is not truly a lower bound, because this definition is still based on the hydrodynamic signals to the lateral line, and not on additional processing that occurs with the neuromast motions and the hair cell filtering. That will be considered in figure 5-31.

Figure 5-30C, D show the results of signal detection threshold based on a high pass filtered noise $(>10 \mathrm{~Hz})$ to the fish lateral line. The first thing to observe is that the differences in signal detection based on flow speed are gone, since higher frequency fluctuations are more homogeneous across flow types. This is a naturally whitening of turbulent motions since larger structures become increasing smaller as energy is removed through dissipation at the higher frequencies. Now the detection threshold increases linearly with an increase in turbulent fluctuations. Recall that the slope of this line is set by the base-noise level inherent for each neuromast. Variations in neuromasts can lead to differences in slope.

Finally, figure 5-31 plots detection threshold based on neuromast motion and hair cell filtering of the hydrodynamic signal. In essence, it is the hypothetical high pass filtering that was just presented in figure 5-30C,D, except that its range starts at $1.5 \mathrm{~Hz}$ (for a time constant of $10 \mathrm{~ms}$ ) rather than at $10 \mathrm{~Hz}$. The base noise level is the root mean square neuromast displacement caused by the oscillating sphere at the stimulus threshold in still water, cited previously. For similar levels of turbulence, the levels of the threshold of detection are now higher than for the $>10 \mathrm{~Hz}$ filtered signal, since there is additional energy from the lower frequencies. This is seen as an increase in the slope of plot $(\mathrm{A})$, and a change in shape and level of the threshold plot in $(\mathrm{B})$. Introducing lower frequencies can reintroduce more variability in fluctuation level.

The signal detection vs. turbulence results (figure 5-30A,C) can be used to evaluate the data from Engelmann et al. (2002) and Chagnaud et al. (2006), who look at the signal masking effects of background flow on the goldfish and trout lateral line. The best fit line of all the data in each plot, (A) and (C), will be used to identify velocity amplitude levels needed to create stimulus signals which equal their levels of 
Sphere velocity amplitude (Uo) needed for signal detection in background flow
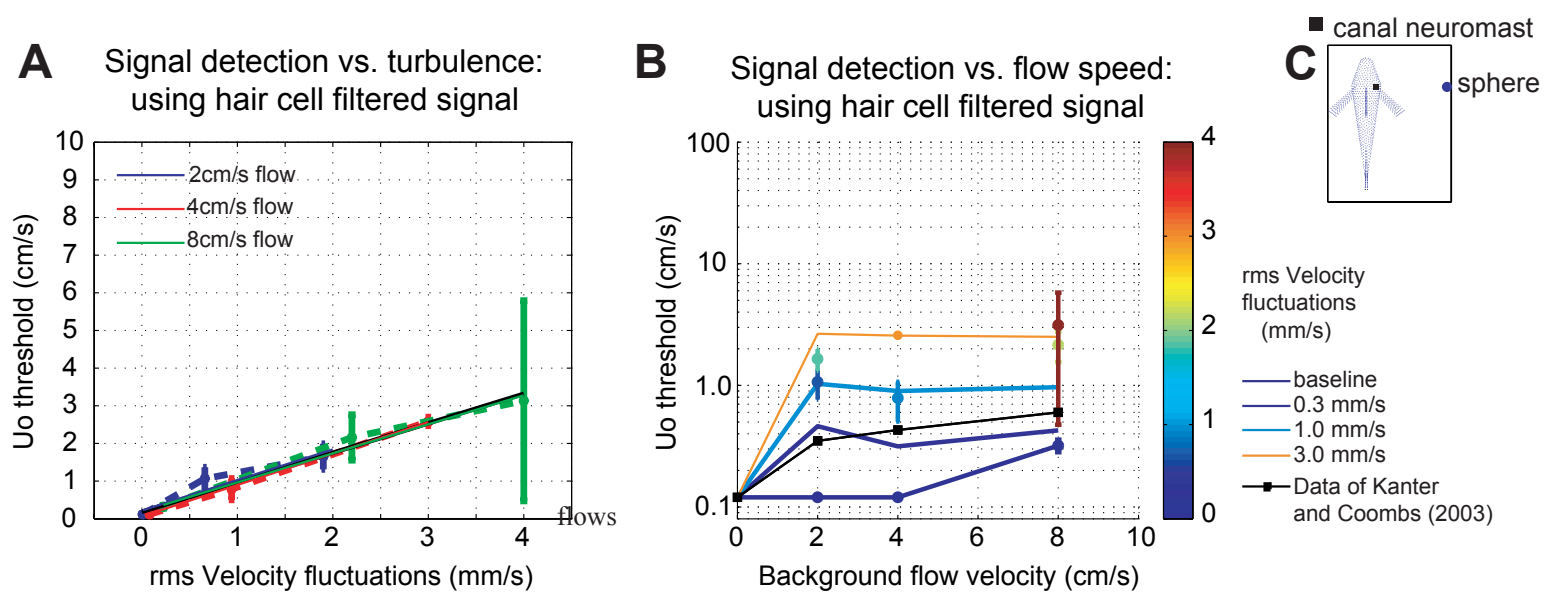

Figure 5-31: Detection thresholds based on the neuromast motions of pressure gradient signals to the canal line, and upon further hair cell filtering. Explanations are as in figure 5-30A,B, except that the noise is based on the root mean square neuromast displacements using the flat plate neuromast model. Likewise, the base-noise is defined as the rms neuromast displacements resulting from the source stimulus in still water at threshold. The sphere had $50 \mathrm{~Hz}$ oscillations and a velocity amplitude of $1.2 \mathrm{~mm} / \mathrm{s}$, and its location relative to the fish and neuromast are shown in $(\mathrm{C})$.

turbulence. Chagnaud et al. (2008) report that their flow set up creates turbulent velocity fluctuations which increase linearly with flow speed.

There are two issues with using the data from this thesis to make inferences about their signal detection results. The first is that their fish were goldfish and trout, whose body shapes are similar to the fins retracted cases presented in this thesis. Since figure 5-29 shows that the fins retracted cases has approximately equal noise levels to the fins extended cases, the same flows used to evaluate noise for the sculpin will be used to evaluate noise for the goldfish and trout. Their lateral lines have a different configuration, so at this point, threshold curves are for illustration purposes only. The second issue is that turbulence generated by the simulations in this thesis will likely have some differences from the turbulence in their experiments. Already one notable difference is that for these simulations, there are patches of turbulence which have an intensity of $10 \%$, but the average turbulence intensity for these more dynamic flows is less than that around the fish. 
The differences that do exist will likely be confined to the low frequency components of the flows. Turbulent fluctuations in natural settings can develop from a flow interaction with the bottom surface, flow around inanimate structures, and flow interacting with aquatic animals. The macro structure of each type of flow will be determined by the specific environmental and boundary conditions. As energy cascades from the larger eddies to the smaller motions, where viscous dissipation occurs, turbulent flows will have more in common. Macro structures will have low frequency pressure and velocity fluctuations which will be unique to each flow, whereas micro turbulence will have a broad high frequency spectrum more in common.

The final step needed is to estimate the base-noise (still/moving) threshold levels for the trout and goldfish. The data of Chagnaud et al. (2006) show that a 7 mm diameter sphere, placed $5-8 \mathrm{~mm}$ from the side of the fish directly next to the neuromast being stimulated, at $50 \mathrm{~Hz}$ oscillations, and having peak-peak displacement amplitudes of $2 \mu \mathrm{m}$, were at or above the threshold level of mean spontaneous spike rate in still and moving water for some anterior canal neuromasts. Other less sensitive canal neuromast required peak-peak displacement amplitudes of at least $30 \mu \mathrm{m}$ to achieve an increase in mean spike rate have a synchronization coefficient of $>0.4$ (phase-locking definition used by Coombs and Janssen, 1990). Assuming maximal sensitivity, this corresponds to a pressure gradient threshold of $2.8 \mathrm{~Pa} / \mathrm{m}$ $\left(\rho A \omega^{2}(a / r)^{3}=1000 *\left(0.5 * 2 \cdot 10^{-6}\right) *(2 \pi * 50)^{2}(3.5 /(3.5+8))^{3}\right)$. This threshold level is 28 times larger than the sculpin $(0.1 \mathrm{~Pa} / \mathrm{m})$, indicating the velocity amplitude of the vibrating sphere in these thesis simulations (see figure 5-1) would have to be $3.4 \mathrm{~cm} / \mathrm{s}\left(28^{*} 1.2 \mathrm{~mm} / \mathrm{s}\right)$ in order to illicit detection for the goldfish anterior lateral line. The less sensitive canal neuromasts would have a pressure gradient 15 times that, or $42 \mathrm{~Pa} / \mathrm{m}$, leading to a stimulus velocity amplitude of $51 \mathrm{~cm} / \mathrm{s}$.

The data of Engelmann et al. (2002) show that a $10 \mathrm{~mm}$ diameter sphere, placed 6-8 $\mathrm{mm}$ from the side of fish directly next to the neuromast being stimulated, at 50 $\mathrm{Hz}$ oscillations, and having peak-peak displacement amplitudes of $10 \mu \mathrm{m} /$, were at or above the threshold level of mean spontaneous spike rate in still and moving water for the posterior canal neuromasts. Assuming maximal neuromast stimulation, this 
corresponds to a pressure gradient threshold of $28 \mathrm{~Pa} / \mathrm{m}\left(\rho A \omega^{2}(a / r)^{3}=1000 *(0.5 *\right.$ $\left.\left.10 \cdot 10^{-6}\right) *(2 \pi * 50)^{2}(5 /(5+8))^{3}\right)$. This threshold level is 280 times larger than the sculpin $(0.1 \mathrm{~Pa} / \mathrm{m})$, indicating the velocity amplitude of the vibrating sphere in these thesis simulations (see figure 5-1) would have to be $34 \mathrm{~cm} / \mathrm{s}\left(280^{*} 1.2 \mathrm{~mm} / \mathrm{s}\right)$ in order to illicit detection for the goldfish and trout posterior lateral line. Since Engelmann et al. did not test stimulus displacement amplitudes less than $10 \mu \mathrm{m} /$, it is not clear that this is the absolute base-noise level. However, it is in keeping with the fact that posterior lateral line canal neuromasts are in general, less sensitive than the most sensitive anterior lateral line canal neuromasts (Coombs and Janssen, 1990).

Figure 5-32 shows the results for the case when the signal threshold is based on the root mean square detrended pressure gradient fluctuations (plot A), and also for the case when the signal threshold is based on the root mean square of the high pass $(>10 \mathrm{~Hz})$ filtered pressure gradient fluctuations. The detrending provides the most basic level of high pass filtering, not unlike what the fish possess. This also serves to make the turbulent flow simulations more similar to the experimental flows, in that it shifts the comparable range a little higher. The high pass filtered $(>10 \mathrm{~Hz})$ set filtering ability lower bounds, perhaps unattainable by the fish. The flows should be even more comparable in this filtered range.

In figure 5-32A, the results show that the less sensitive anterior canal neuromast in the goldfish, and the posterior canal neuromasts of the goldfish and trout, have base-noise threshold levels which are well above the noise of the background turbulence until the flow reaches $12 \mathrm{~cm} / \mathrm{s}$. This indicates there would be no signal masking of the stimulus signal by these canal neuromasts, in agreement with the experimental results of both Engelmann et al. and Chagnaud et al. However, the more sensitive anterior canal neuromast of the goldfish would show signal masking of the stimulus at even the slowest of background flows, in contradiction to the experimental results of Chagnaud et al. Therefore, there is some additional filtering.

Figure 5-32B shows that the filtered pressure gradient signal, representing the higher frequency spectral components of the turbulent flows, serves to reduce the effects of turbulence on signal detection. Now the more sensitive anterior canal neu- 
romast of the goldfish would not show signal masking until about $8 \mathrm{~cm} / \mathrm{s}$. The raster plots for this sensitive neuromast (Chagnaud et al., 2006, figure 2) do show a slight degree of signal masking of the $10 \mathrm{~cm} / \mathrm{s}$ flow at a stimulus level slightly above threshold (as defined earlier), so these results are plausible.

The effect of turbulence on signal detection, as compared with the role of filtering and of the base-noise threshold level for goldfish and trout
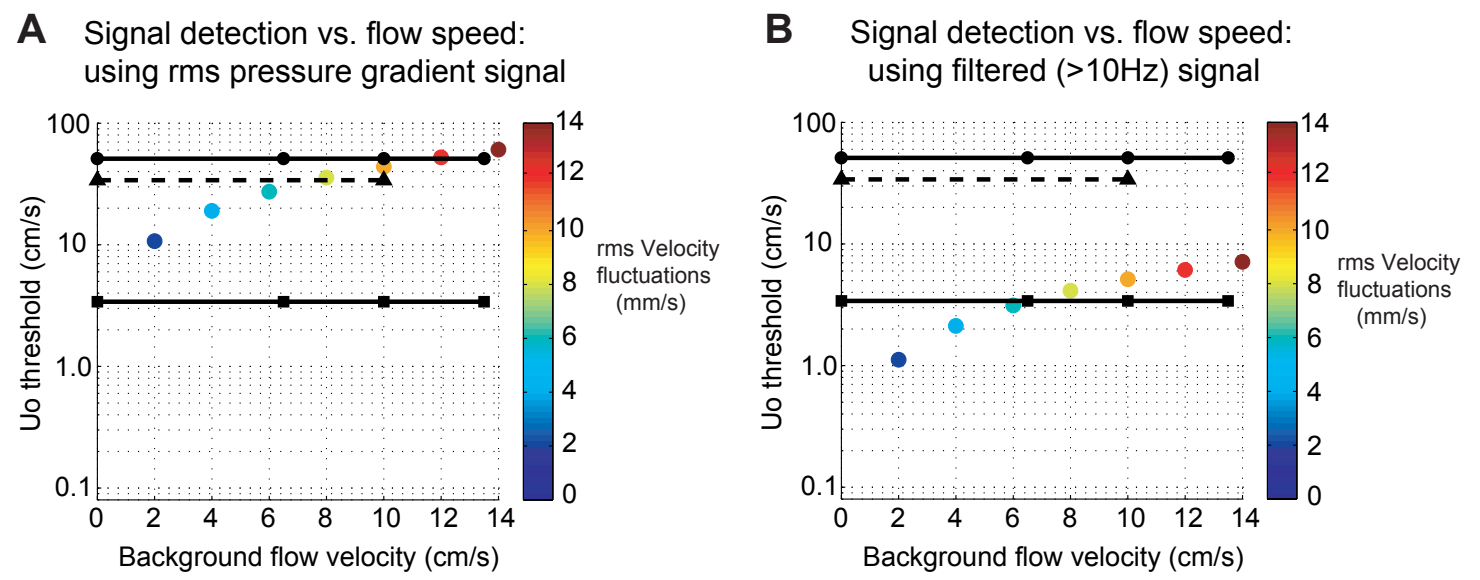

Figure 5-32: Extrapolating the results of figure 5-30A,C for use with data from Engelmann et al. (2002) and Chagnaud et al. (2006). The level of signal threshold, as determined by the level of background turbulence, was determined for the sculpin, and is used for illustrative purposes for the trout and goldfish (see text for explanation). The levels of turbulence are taken from Chagnaud et al. (2008), and then interpolated using the best fit lines of the data in plots $(\mathrm{A})$ and $(\mathrm{C})$ of figure 5-30. The base-noise level is calculated using experimental data for the goldfish and trout (see text), and calibrated to match the stimulus velocity amplitudes of a sphere in the location used in this thesis. Key: colored dots indicate turbulence level; black squares and black dots - the base-noise level for two different anterior lateral line canal neuromasts of the goldfish (Chagnaud et al, 2006); black triangles - base-noise level of the posterior lateral line canal neuromasts of the goldfish and trout (Engelmann et al, 2002); (A) Threshold determined by the root mean square detrended pressure gradient signal to the sculpin lateral line; (B) Threshold determined by the root mean square high pass filtered $(>10 \mathrm{~Hz})$ pressure gradient signal to the sculpin lateral line. Location of sphere, and parameters used for calibration with sculpin fish are given in figure 5-30. 


\subsection{Discussion}

The core question of this thesis is how fish are able to detect the oscillations of a dipole signal whose flow induced signal to the lateral line system of fish can be orders of magnitude less than the motions of the background flow. The actual behavioral evidence of this comes from the sculpin strikes on a $50 \mathrm{~Hz}$ vibrating sphere in flow speeds ranging from 0-8 cm/s (Kanter and Coombs, 2003). The $3 \mathrm{~mm}$ sphere, placed an average distance of $56 \pm 9 \mathrm{~mm}$ from the longitudinal midline of the fish, was increased in $5 \mathrm{~dB}$ increments until the fish made a strike on it or movement towards it.

The hydrodynamic motions induced by the vibrating sphere stimulate both the superficial neuromasts and the canal neuromasts. The previous chapter examined how the superficial neuromasts respond to the boundary layer flow induced by the dipole stimulus in still water. These stimulus velocities are orders of magnitude smaller than the background flow speeds. The pressure gradients to the canal lateral line, introduced by the oscillating sphere at the detection threshold level, are about 0.1 $\mathrm{Pa} / \mathrm{m}$ for the sculpin. This thesis showed that in even in laminar background flows, the mean pressure gradients to these sensitive neuromasts begin at about $8 \mathrm{~Pa} / \mathrm{m}$ and increase to over $100 \mathrm{~Pa} / \mathrm{m}$.

This apparent disparity in signal strengths just lends support to what is already well known, that the lateral line system is able to remove the mean components of the hydrodynamic signals. One point that must be emphasized is that there is a difference between filtering the mean hydrodynamic components and being unaffected by the mean hydrodynamic components. Both the superficial and canal neuromasts

physically respond to these mean components by being deflected or displaced. The small amplitude oscillations from the dipole stimulus are superposed onto these mean hydrodynamic signals (along with whatever noise exists), and so the corresponding neuromast displacements can indeed be orders of magnitude smaller than the overwhelming displacements resulting from the mean components.

The first act of filtering is physical, in the boundary layers that develop on the 
surface of the fish or inside the subdermal canals. For the superficial neuromast, the effect is high pass filtering and for the canal neuromast, it is low pass filtering (see figures 3-12 and 3-9). The second act of filtering is through the physical responses of the neuromasts to these boundary layer and subdermal canal filtered velocities. The material properties of the neuromast, and the density and viscosity of the fluid, determine how the hydrodynamic signals will be filtered. The overall system effect of surface boundary layer, subdermal canals, and neuromast motion dynamics, leads to high pass filtering for superficial neuromasts and all pass filtering for the canal neuromasts, up to their respective cut off frequencies (see figures 3-14 and 3-11).

For superficial neuromasts, the surface boundary layer and neuromast mechanics work to reduce the mean free stream velocities, but as figure 3-14 shows, the velocities at all frequencies are also reduced. There is a best sensitivity around $10 \mathrm{~Hz}$, but not enough to compensate for the orders of magnitude difference between stimulus induced velocities and background flow velocities. For canal neuromasts, the subdermal canal and neuromast mechanics also reduce the effects of the mean pressure gradient signal created by the free stream flow, but equally reduce signals at all frequencies. This also cannot account for the detection abilities of the fish.

A second argument that the physical filtering process cannot account for the signal detection abilities of the fish has to do with saturation effects that should prevent detection, but do not. The neuromast models developed in this thesis make predictions as to how the neuromast will physically respond to its hydrodynamic environment. For the higher speed flows, the resulting displacements can be well past the saturation mark of approximately $100 \mathrm{~nm}$. The fact that the fish does respond to the small amplitude stimulus even in this range is the best evidence that the mechanism for handling the mean component is internal, in the hair cells, and not external through the boundary layer, canal, and neuromast motion filtering effects.

The hair cell has been shown to possess the mechanism that deals with the mean deflection of the hair cells. The gating apparatus, which has links between stereocilia, is tensioned to open and close gates which allow for the exchange of ions which controls the current through transduction channels (Eatock, 2000). Sustained deflections, such 
as would be caused by the mean components of the hydrodynamic signals, can be adapted to through the repositioning of hair cell links to ease the tension. The fact that it is not the absolute defection of hair cells that controls signal transduction, but rather the relative tension of the links between stereocilia, would seem to allow the effect of the mean signal component to be completely removed since this is a differencing mechanism. However, there are physical limits to adaptation, and in severe deflections there is incomplete adaptation (Eatock et al., 1987). Therefore, the mean hydrodynamic components cannot be completely discounted. At high enough flow speeds, all neuromasts should eventually saturate. This may not occur within normal swimming regimes, but perhaps it might occur during attack and evasive maneuvers for fast swimmers.

The fact that bulk flow stimulates superficial neuromasts almost equally, regardless of flow direction (Chagnaud et al., 2008), further supports the notion that it is not the absolute deflection of hair cells which is the transduction mechanism of the hydrodynamic signal. Superficial neuromasts have preferred directions of stimulation (Flock and Wersall, 1962), and changing the direction of the flow should cause the neuromasts to switch from stimulation to inhibition, or vice versa. Instead, the hair cells adapt to the bulk flow, but not to the a.c. fluctuations of the flow (Chagnaud et al., 2006).

Based on the experimental work of Eatock et al. (1987), the low pass filtering effects of the hair cell transduction process can be crudely quantified. Hair cells were manually forced to move according to a step function input, and the hair cell currents and potentials were measured to have exponential function outputs. Figure 5-11 shows the filtering effects that would produce exponentially decaying outputs, based on the biological range of time constants measured. What it shows is that the hair cell transduction process has a significant high pass filtering effect on the lowest frequencies $(¡ 1.5 \mathrm{~Hz})$. This has been observed in neurological studies, where the bulk flow (and therefore neuromast motions) had spectral peaks below $5 \mathrm{~Hz}$, whereas the spectra of firing rates had peak frequencies in the range of 3-8 Hz (Chagnaud et al., 2008). 
The next question to address is whether or not the hair cell transduction process is the final step of filtering required in order for the small amplitude stimuli to be detected against larger background noise. In other words, based on the boundary layer, canal, neuromast motions, and hair cell transduction process, can the small amplitude stimulus signals be detected in background flow noise? If not, it suggests that further processing of the signals must occur in the brain of the fish.

What would constitute an answer to that question? There are two methods described in this thesis for signal detection: an increase in the mean spike rate, and phase-locking. If after filtering, it can be shown that the presence of the signal could increase the root mean square pressure gradient fluctuations a significant amount above the noise level, or it can be shown that phase-locking to the stimulus spectral peak is possible, then no further filtering is required.

The increase in mean spike rate, and phase-locking, must occur at the levels of the source stimulus identified experimentally. In studies involving background flows and a vibrating sphere, Engelmann et al. (2002) present neurological results for the posterior lateral line, Chagnaud et al. (2006) present neurological results for the anterior lateral line, and Kanter and Coombs (2003) present behavioral results. It is difficult to know the actual flow conditions in which the experiments occurred. Further experimental studies by these authors, without the presence of the vibrating sphere but in otherwise similar flow conditions, provide valuable insight. Chagnaud et al. (2008) found a linear increase in rms velocity fluctuations with increase in flow rate, yielding a fairly constant turbulence intensity of about $10 \%$. They used one upstream and one downstream collimator, with the tank supported on a vibrationisolated table, and the flow was driven by propeller coupled to a DC motor. Coombs et al. (2007) placed a flow tank of equal size to the Kanter and Coombs (2003) experiment inside a larger recirculating, oval-shaped flume, driven by conveyor belt and rotating paddles. They used two collimators placed upstream of the fish, and a mesh screen placed downstream, as in the Kanter and Coombs experiment. The level of turbulence is unknown, but the mean flow measurements of velocity and vorticity are provided, and can be qualitatively compared directly with the laminar (figure 5-3) 
and turbulent (figure 5-4) flow results of the simulations in this thesis.

In the laminar flow simulations of this thesis, the mean pressure gradient component at each neuromast location remains constant in time (see figures 5-12 - 5-14). This component is almost certainly removed in all of the flow cases presented, since the resulting neuromast motions show displacements under $150 \mathrm{~nm}$ for all neuromast models except the spherical neuromast model responding to $8 \mathrm{~cm} / \mathrm{s}$ flow. With displacements that are not too excessive, adaptation is more complete.

The power spectral density plots for those figures show the dominant spectral peak is at $50 \mathrm{~Hz}$, indicating that phase-locking would correlate with detection. For the 2 and $4 \mathrm{~cm} / \mathrm{s}$ flows, the stimulus source velocity amplitude was $1.2 \mathrm{~mm} / \mathrm{s}$, identical to the still water stimulus level. In $8 \mathrm{~cm} / \mathrm{s}$ flow, the stimulus level shown is $6.0 \mathrm{~mm} / \mathrm{s}$, but as figure 5-15 shows, only a stimulus velocity amplitude of $4.3 \mathrm{~mm} / \mathrm{s}$ is needed to make the $50 \mathrm{~Hz}$ signal the dominant spectral peak. Figure 5-31 presents detection threshold levels needed to increase the spike rate above the background flow noise. The baseline results, which represent the noise in the laminar flow simulations, lie below the stimulus threshold levels as calculated by Kanter and Coombs (2003).

The experimental results of Kanter and Coombs (2003), on the other hand, show signal masking even at the $2 \mathrm{~cm} / \mathrm{s}$ flow speeds. If their flows conditions were laminar, there should not have been any signal masking until the $8 \mathrm{~cm} / \mathrm{s}$ flow speed (see baseline results, figures 5-30B and 5-31B). The experimental conditions of Coombs et al. (2007), which were meant to match those of the previous experiment, do show evidences of turbulence found in the presence of alternating streaks of vorticity that move past the sculpin at each of the flow speeds $(2,4$, and $8 \mathrm{~cm} / \mathrm{s})$. Even if we did know the turbulence of this latter experiment, the previous experiment had the flow generated in a different manner, through the use of a motor-driven impellor. The turbulence levels would be different than under the paddle driven flow.

For the turbulent flow simulations in this thesis, the hair filtered responses to the neuromast motions were not able to fully remove the low frequency hydrodynamic signals (see figures 5-17A, 5-19A, 5-21A, 5-23A, 5-25A, and 5-27A ). Chagnaud et al. (2008) indicate that phase-locking would occur for these dominant, low frequency 
oscillations. They also indicate that phase-locking occurs well before an increase in the mean spike rate. Therefore, under these flow conditions, the sculpin should not be able to detect the vibrating source stimulus at the velocity amplitudes used (3.5 $\mathrm{mm} / \mathrm{s}$ for $2 \mathrm{~cm} / \mathrm{s}$ flow, $4.3 \mathrm{~mm} / \mathrm{s}$ for $4 \mathrm{~cm} / \mathrm{s}$ flow, and $6.0 \mathrm{~mm} / \mathrm{s}$ for $8 \mathrm{~cm} / \mathrm{s}$ flow).

At each of the flow speeds, and with each of the velocity amplitudes used in these turbulent simulations, there does exist the possibility for detection if the brain were to further process the signals. For each of the flow speeds, there were two levels of turbulence reported. For the lower levels of turbulence at each flow speed, the spectral peak at $50 \mathrm{~Hz}$ does rise above any peaks in frequency proximity. The results of the $>10 \mathrm{~Hz}$ high pass filtering demonstrate that at these lower turbulence levels, the $50 \mathrm{~Hz}$ stimulus amplitude is recoverable (see plots $\mathrm{C}$ and $\mathrm{E}$ for each of the figures mentioned above). At the higher levels of turbulence, these stimulus source oscillations are indistinguishable from the turbulent fluctuations, and are not recoverable. This is confirmed in the spectral plots, where the noise level at nearby frequencies is equal to or above the spectral energy level at $50 \mathrm{~Hz}$ (see plot B of each figure of the higher turbulent flows).

Since the spectral plots of turbulence for the Kanter and Coombs (2003) experiment is unknown, it is not possible to determine if these turbulent flow simulations are representative of their flows. The laminar flow results of this thesis show that further processing by the brain would not be needed at the stimulus amplitudes used in their experiments. Therefore if their turbulence levels were very low, or if their flows had low amplitude low frequency oscillations, then the results of this thesis suggest that no further processing is need by the sculpin. The hair cell filtering is sufficient. On the other hand, if there flows have similar levels of turbulence to these simulations, then the results suggest that further processing by the brain is needed.

If the flows of Kanter and Coombs (2003) are similar to the flows of Chagnaud et al. (2008), since both were generated by motor driven propellers, then the turbulence levels should have similar spectral characteristics. This means that the dominant flow energy is in the low frequency range (below $5 \mathrm{~Hz}$ ). For the goldfish, the neural responses showed evidence of high pass filtering, with peaks occurring in the $3-8 \mathrm{~Hz}$ 
spectral range. These neural responses occurred even without an increase in mean spike rate, which lead Chagnaud et al. to postulate that it is not the spike rate codes that are most relevant to the fish, but the temporally modulated information. This would also suggest that the purpose of the hair cell is to pass on the most relevant information to the brain - the hydrodynamic activity within a selected frequency range.

Using the data of Engelmann et al. (2002) and Chagnuad et al. (2006), it is inconclusive as to whether or not further filtering is required by the brain. It appears that for some neuromasts, filtering is not needed, while other more sensitive neuromasts might require it. When the source stimulus which is above their base-noise level of the less sensitive neuromasts, detection would occur, since the stimulus signal will be well above the background noise levels tested. Therefore, both mean spike rate and phase-locking would occur on the prey stimulus, indicating that the hair cell filtering is all that is required for those fish, under those conditions. When the source stimulus signal is only at the base-noise level of the sensitive neuromasts, the background flow noise would mask the signal unless additional filtering occurs.

Since the canal neuromasts for the trout and goldfish were not modeled in this thesis, the filtering effects of the neuromast motions and hair cell transduction process can only be surmised to produce results that fall between what was plotted in figure 5-32A and 5-32B. Secondly, the velocity thresholds calculated were based on the turbulence in these particular flow simulations. Different flows, and different filtering capabilities, will alter the threshold vs. turbulence slopes plotted in figures 5-30A,C. However, trout swimming in streams can experience turbulence levels much higher than in the flows generated in the experiments (up to $70 \mathrm{~mm} / \mathrm{s}$; Hanke, 2001). At these levels of turbulence, there might very well be significant masking for all types of neuromasts. Therefore, these flows are not conclusive in determining if further processing is required.

For the trout and the goldfish, it appears that having a high base-noise threshold level can be advantageous for them. Since the trout and goldfish swim in waters that can have higher levels of turbulence, their base-noise levels for their canal neuromasts 
need to be high enough not to be affected by the background noise. The consequence is that they cannot be sensitive to small amplitude stimulus vibrations, unlike the sculpin. The types of prey that would attract them would be larger than the types of prey eaten by the sculpin. 


\section{Chapter 6}

\section{Conclusion}

\subsection{Summary of results}

What began this thesis was the observation that fish have a remarkable ability to detect small desirable signals in the midst of much larger background noise. They of course have at their disposal the senses of sight, hearing, and smell, but perhaps one of their most useful senses is touch. For the fish, it is their lateral line system, consisting of tiny hairs either on the surface or in subdermal canals, which provides for their sense of touch by directly linking the outside world of fluid motion and vibration to what will become a corresponding signal representation of that world in their brains.

The fish lateral line has been studied for over 150 years, but there is always room for greater understanding. In the last 30 years, many advances in the study of the lateral line have occurred, in particular because of the great advances in technology which firstly enable more precise measurements of the lateral line, and secondly enable better modeling and computation. The import of this thesis is that it enables cause and effect to be examined in component form when addressing the signal detection ability of fish.

This thesis directly utilizes our modern day ability to resolve simple fluid dynamic problems by fully solving the Navier-Stokes equation with moderate boundary conditions. To the authors knowledge, this is the first time that complete time-dependent, 
viscous solution for a vibrating sphere next to a fish, has been solved. This is important for a number of reasons. The vibrating sphere creates an oscillating dipole flow field, which is thought to represent some of the more important signals that a fish would try and detect. Disturbances created by swimming prey will locally create a complex signal, but further away this signal will decay to simpler quadrapole and dipole representations as higher multi-pole components die out. When there is an oscillatory nature to these signals, a corresponding oscillatory boundary layer of reduced velocity and of a distinct profile will develop on the surface of the fish. The superficial neuromasts are often either mostly or fully submersed inside this signal induced boundary layer.

This thesis develops an approximation to what the dipole induced, oscillatory boundary layer velocity profile, will look like. An analytic solution is developed which is accurate at points of maximal surface tangential velocity for the case when the surface is flat. This was accomplished by noting that next to a surface, flow is forced to alter course to move in a direction which is tangential to the wall. For a dipole-wall signal interaction, the flow field is not uniform, and does contain localized regions which violate this assumption as flow is forced to either stagnate or eject in those regions. However, for much of the rest of the flow along the wall, the flow is forced tangential and the approximation holds. Knowledge of the flow within the oscillating dipole induced boundary layer allows for direct determination of the strain rate, a relative measure of the signal strength to the superficial neuromast, and of the shear stress at the surface.

One key finding is that an oscillatory dipole induced flow field retains much of its potential nature outside of the induced surface boundary layer. Potential flow theory has been used to model the oscillating dipole signal in an unbounded medium, and in some cases, with axisymmetric bodies present. This thesis extends previous work by using realistic fish body shapes, which have body parts such as a dorsal fin, a tail, and pectoral fins. In still water conditions, the presence of body parts had a relatively small effect on the received dipole signal, with distortions confined to regions of fin insertion points. The fact that viscous effects are contained within a 
surface boundary layer, whose depth is inversely proportional to the square of the signal frequency, means that most of the flow field around the fish remains of a potential nature.

Experiments have suggested that the two lateral line systems, the surface superficial neuromast system and the subdermal canal neuromast system, do not have equal importance for prey detection and localization. This thesis was able to quantify these differences in detection ability in the case of an oscillating dipole stimulus. In particular, the detection threshold for superficial and canal neuromasts are determined for the Mottled Sculpin, using the data obtained from neurological and behavioral studies. For a nearby vibrating sphere, the induced signal strength relative to the measured threshold levels revealed that the canal lateral line is much more sensitive than the superficial lateral line system. At some frequencies, the canal lateral line system was over 100 times more sensitive than the superficial lateral line system.

This thesis also developed superficial and canal neuromast models specific to the Mottled Sculpin. It built upon generic models (van Netten, 2006; Humphrey et al., 2001) by including additional hydrodynamic terms and material/structural terms which better matched measured neurological results. This enables the mechanical transduction component of the signal encoding process to be examined in isolation for its contribution to signal detection and filtering. In a similar manor, the physical filtering process which occurs from the induced boundary layer flow, and from the induced flow within the subdermal canal, can be studied in isolation and in combination with the mechanical filtering properties of the neuromasts.

This thesis highlights the importance of the physical response of the superficial and canal neuromasts to oncoming flow. Experiments have shown that superficial neuromasts become saturated in oncoming flow, but that the canal lateral line systems display high pass characteristics which keep it from becoming saturated. These observations have been obtained from neurological studies, and they have not isolated which components of the signal encoding process cause these results.

Both the superficial and canal neuromasts have the potential to be deflected to the point of saturation when oncoming currents increase to high enough flow rates 
$(10+\mathrm{cm} / \mathrm{s})$. The mean flow past the surface of the fish creates a boundary layer which grows in height along the fish, originating at the point of contact on the nose. For swimming fish, this boundary layer is largely kept intact and possibly controlled (Anderson et al., 2001), but for gliding fish or benthic fish such as the sculpin, the boundary layer grows to the point of separation and can become turbulent in nature. The superficial neuromasts will exhibit oscillations around a mean point of deflection, determined by the strength of the mean flow and the a.c. fluctuations present. Likewise, changes in the speed of flow around the fish body, due to its curvature, cause accelerations which signal the canal lateral line system. Depending on the location of the canal neuromasts on the body, this can result in deflections that should saturate the neuromasts (whereas other neuromasts can be located in regions of minimal stimulation).

The fact that the physical deflection of superficial neuromasts, and the physical displacement of canal neuromasts, does not lead to saturation, shows that the physical and mechanical filtering of the boundary layer flow, subdermal canal flow, and neuromast motions, are not ultimately responsible for signal detection. Instead, they point to internal filtering mechanisms of hair cells and of signal processing in the brain.

This thesis is able to show that, based on the known hydrodynamic signals to the lateral line system, signal adaptation at least occurs at the level of the hair cell. Material links between stereocilia in the hair cells tension the opening and closing of gates, controlling the flow of ions in and out of the hair cell. Essentially this acts as a physical differencing mechanism, which for short response times of 10-200 milliseconds acts as a high pass filter (effectively removing frequencies below $1.5 \mathrm{~Hz})$. Between the physical filtering of the boundary layer flow, subdermal canal flow, neuromast motions, and hair cell gating, the lateral line system is tuned to detect a.c. fluctuations present in the flow.

The motions of the neuromasts also highlight the importance of a.c. fluctuations. Results obtained in this thesis show that the neuromasts respond most sensitively to quick changes in the temporal flow field by having overshoots of motion. The question 
is how the fish can determine what overshoots are most important.

To understand how fish distinguish important a.c. fluctuations, this thesis used increasing levels of turbulent flow to examine signal masking of known levels of stimulus evoking responses from the sculpin. Analysis in the spectral domain revealed that the stimulus signal must have spectral peaks which are above all other peaks in order for phase locking to occur. However, evidence suggests that phase locking will occur on the dominant low frequency flow signals, and not on the higher frequency stimulus signals.

If fish can detect these high frequency stimulus signals, while concurrently phase locking onto low frequency flow noise signals, then the best explanation of results is that further signal processing occurs beyond the hair cell, and possibly in the brain itself. This was not determined conclusively in this thesis because there was not a way to absolutely link levels of turbulence in this thesis with the levels of turbulence existing in experiments which provide the neurological and behavioral signal detection results.

\subsection{Future research}

The methodology of this thesis was to analyze component features of the stimulus signal, of the physical and mechanical transduction process, and of the hair cell transduction process in order to understand how signal encoding takes place. This thesis has laid the foundation for future research on the signal detection and signal processing abilities of fish using their lateral line system. New experiments which link signal detection results with spectral decomposition of turbulent flows will allow for positive determination of where signal detection ultimately occurs.

The importance of transient signals needs to be studied in more depth. The possibility for the fish to utilize spatial and temporal information from multiple neuromasts could enable the fish to track signals as the peak amplitudes change location on their body. The ability for fish to school together could be explained and quantified, and prey detection and wake tracking would be best exploited in a spatial and temporal 
integration of information.

Another important area of research would be to understand how the superficial and canal neuromasts respond to the swimming, moving, and breathing motions of the fish itself. This becomes noise, which the fish would likely want to filter. On the hand, it has been suggested that superficial neuromasts might have their importance in helping to monitor and regulate the surface boundary layer that develops as the fish swims (Anderson et al., 2001; Windsor, 2008). 


\section{Appendix A}

\section{An oscillating cylinder in still water: THEORY}

For an incompressible Newtonian fluid of uniform temperature and constant viscosity, in a non-rotating frame, the Naiver-Stokes equation is

$$
\frac{\partial \mathbf{u}}{\partial t}+\mathbf{u} \cdot \nabla \mathbf{u}=-\frac{1}{\rho} \nabla p+\nu \nabla^{2} \mathbf{u}+\frac{1}{\rho} \mathbf{F}
$$

where $\mathbf{u}=(u, v, w)$ is the vector velocity quantity, $p$ is the pressure, $\rho$ is the density of water, $\nu$ is the kinematic viscosity, and $\mathbf{F}$ is the vector body force per unit volume. When the fluid is assumed continuous to the smallest scale, and mass is conserved, there results what is called the equation of continuity,

$$
\frac{D \rho}{D t}+\rho \nabla \cdot \mathbf{u}=0
$$

where $D / D t$ is the total derivative. These two equations are sufficient to capture all of the relevant fluid dynamics for the case of a circular cylinder performing periodic oscillations. However, it will be possible to make further simplifications when considering the motion chosen to be replicated in the numerical simulations.

The first simplification will be to assume that the cylinder is of infinite vertical height in the z-direction, and that the oscillations will take place perfectly in the $\mathrm{x}-\mathrm{y}$ 
plane. As long as the flow remains laminar, the flow in the z-direction will be zero, and the problem is two-dimensional. As such, the body force $(\mathbf{F})$, which in general is the gravitational force acting in the z-direction, can be discounted.

There are two parameters which effectively determine whether or not the flow will remain laminar over the oscillating cylinder, the Keulegan-Carpenter number, $K C$, and the Stokes number, $\beta$. These number are defined for flows which oscillate past a stationary cylinder, but as Lighthill (1978) has explained, this is equivalent to a stationary flow with the cylinder undergoing oscillations. The Keulegan-Carpenter number is $K C=2 \pi A / d$, with $A$ the amplitude of motion and $d$ the diameter of the cylinder. Stokes number is $\beta=f d^{2} / \nu$, with $f$ being the frequency of oscillation and $\nu$ being the kinematic viscosity of the fluid. Tatsuno and Bearman (1990) show that these two parameters effectively capture the dynamics of vortical shedding patterns, which seem to fall into 8 regimes. For each $\beta$ regime, the lowest $K C$ values lead to laminar flow, ie. no vortical shedding. For example, $K C$ can be as high as about 6 when $\beta<10$, whereas $K C$ can only be as high as about 3 when $\beta>50$.

When the flow past an oscillating cylinder remains laminar, there is a steady (mean, non-oscillating) secondary flow that results, called acoustic streaming. Lighthill (1978) has explained that two types of attenuation, attenuation of acoustic energy and attenuation due to friction, allows for excess momentum flux to force steady streaming. For the cylinder, the steady streaming occurs outside of the Stokes boundary layer, which approximately extends a distance of $5(\nu / \omega)$, with $\omega=2 \pi f$ being the angular frequency. In general, the Stokes boundary layer is much smaller than the diameter of the cylinder, and so the steady streaming at the edge of the Stokes boundary layer can be thought of as a steady velocity fluid slipping past the surface of the cylinder. Lighthill (1978) says this mean 'slip velocity' is calculated through Rayleigh's Law, given as

$$
\bar{u}_{s}=-\frac{3}{4} \omega^{-1} U_{c}(X) U_{c}^{\prime}(X)
$$

where $X, Y$ denote a new coordinate system with $X$ tangential and $Y$ perpendicular to the surface of the cylinder, and $U_{c}(X)$ is the tangential velocity of the undisturbed 
flow relative to the location $X$ on the surface of the cylinder. This law is valid as long as $\partial u_{c} / \partial X+\partial v_{c} / \partial Y$ is independent of $Y$, where $u_{c}$ and $v_{c}$ are the tangential and perpendicular velocities in the new coordinate system relative to the surface of the cylinder.

There are two regimes of acoustic streaming: slow creeping flow spread over a large region, and high speed flow in a region close to the cylinder body that collide and are emitted as jets along the axis of oscillation . The two parameters previously described, the Keulegan-Carpenter number and Stokes number, place the flow into the acoustic streaming regimes. Within these regimes, the Reynolds streaming number, $R_{s}=U_{o}^{2} / \omega \nu$, determines whether the streaming will result in creeping flow or high speed jets. This number is very similar to Stokes number, as will be seen after defining $U_{o}$. If the center of the cylinder has its position given by

$$
X_{c}(t)=A \sin (\omega t)
$$

with $A$ its amplitude of motion and $t$ the time, then the velocity of the cylinder is given by

$$
V_{c}(t)=U_{o} \cos (\omega t)
$$

where $U_{o}=A \omega$ is the maximum velocity of the fluid at the surface of the cylinder. Then the Reynold's streaming number can be rewritten as $R_{s}=2 \pi f A^{2} / \nu$, showing the differences from Stokes number to be a factor of $2 \pi$, and the characteristic length scale being the amplitude of motion, $A$, rather than the diameter, $d$. When $R_{s} \ll 1$, creeping flow results, and when $R_{s} \gg 1$, high speed jets are emitted (Riley, 1965).

Revisiting the assumption that the fluid is incompressible, it needs to be shown that the characteristic velocities of the fluid are much smaller than the speed of sound (or equivalently the propagation of density changes) in the medium. For incompressibility to be a valid approximation, $U_{o} \ll c$, or $A \ll c / \omega$, where $c$ is the speed of sound in water. For oscillations of maximum frequency $f=100 \mathrm{~Hz}$ and the speed of sound in water $c=1500 \mathrm{~m} / \mathrm{s}$, the amplitude of oscillation must be much smaller than $2.4 \mathrm{~m}$. This will be easily met for the cases of study, where the amplitude of 
oscillation is generally much less than the radius of the cylinder, $a$, of approximately $1 \mathrm{~cm}$ diameter.

In the numerical simulations, incompressibility will be forced, which is equivalent to saying that the speed of sound in the medium is increased to infinity, or to saying the the density throughout the fluid will remain constant, making $D \rho / D t=0$. Therefore, in numerical simulations, assuming incompressibility results in using an exact equation,

$$
\nabla \cdot \mathbf{u}=0
$$

Finally, for the cases of study the amplitude of motion will be very small, resulting in a velocity that is very small. It needs to be shown that the velocity squared term $\mathbf{u} \cdot \nabla \mathbf{u}$ will be much smaller than the remaining terms and therefore able to be neglected. When substituting each term of the Navier-Stokes equation with its characteristic velocity, time, length, or pressure value multiplied by a non-dimensional term, the Navier-Stokes equation can be rewritten as a series of special coefficients multiplying non-dimensionalized $(N D)$ units of $O(1)$.

$$
S \cdot\left(\frac{\partial \mathbf{u}}{\partial t}\right)_{N D}+(\mathbf{u} \cdot \nabla \mathbf{u})_{N D}=-2 E u \cdot(\nabla p)_{N D}+\frac{1}{R e} \cdot\left(\nabla^{2} \mathbf{u}\right)_{N D}
$$

where the Strouhal number $S=L / U_{o} T$, the Euler number $E u=P_{o} / \frac{1}{2} \rho U_{o}^{2}$, and the Reynolds $R e=U_{o} L / \nu$. A characteristic length $L$ would be the diameter of the cylinder, $2 a$, so that the Strouhal number would be $O\left(2 a \omega / 2 \pi U_{o}\right) \approx O(a / A) \gg 1$. Without yet proving that the pressure in the vicinity of the cylinder is $O\left(\rho a \omega U_{o}\right)$, the Euler number is much larger than 1. Finally, the Reynolds number must be much smaller than 1 , so that means $2 a A \omega \ll \nu$, limiting the amplitude of oscillation to $A \ll \nu / 2 a \omega$. The simplified momentum and continuity equations for which Stokes (1851) solved this problem analytically are

$$
\frac{\partial u}{\partial t}=-\frac{1}{\rho} \frac{\partial p}{\partial x}+\nu\left(\frac{\partial^{2} u}{\partial x^{2}}+\frac{\partial^{2} u}{\partial y^{2}}\right)
$$




$$
\begin{gathered}
\frac{\partial v}{\partial t}=-\frac{1}{\rho} \frac{\partial p}{\partial y}+\nu\left(\frac{\partial^{2} v}{\partial x^{2}}+\frac{\partial^{2} v}{\partial y^{2}}\right) \\
\text { and } \quad \frac{\partial u}{\partial x}+\frac{\partial v}{\partial y}=0 .
\end{gathered}
$$

When the boundary conditions are that the fluid at the surface of the oscillating cylinder move with the cylinder, and be at rest at infinite distance from the cylinder, there results a system of equations for which certain integrations do not exist with a finite number of terms. Though the solution for an oscillating sphere is quite straight forward, the solution for an oscillating cylinder is not, and it eluded Stokes for 'some time.' It was not until he was working on the solution to a definite integral for a different problem that he realized it would apply to the case of an oscillating cylinder.

Stokes main reason for solving these cases (oscillating sphere and cylinder) was to account for puzzling discrepancies between theory and experiment found for the period of a pendulum undergoing small oscillations (as worked on by Dubuat, Bessel, Poison, Baily, and others). He was looking to theoretically explain what corrections to the period should be expected for pendulums made from different materials, shapes and sizes, placed in mediums other than a vacuum (such as air or water). His explanation is the theoretical development of viscosity, which he calls 'the internal friction of a fluid.'

Stokes gives the framework of equations, and accounts for the most difficult of constants, but does not solve for the velocity or pressure fields. Even Lamb does not offer a solution for the case of an oscillating cylinder, but defers to Stokes' work. For numerical validation, both the velocity and pressure fields are needed analytically, and fortunately they are easily obtained from where Stokes left off. Putting the governing equations into polar coordinates, Stokes obtained (with symbols changed for this thesis)

$$
\begin{gathered}
v_{r} r d \theta-v_{\theta} d r=d \chi \\
\left(\frac{\partial^{2}}{\partial r^{2}}+\frac{1}{r} \frac{\partial}{\partial r}+\frac{1}{r^{2}} \frac{\partial^{2}}{\partial \theta^{2}}\right) \chi_{1}=0
\end{gathered}
$$




$$
\begin{gathered}
\left(\frac{\partial^{2}}{\partial r^{2}}+\frac{1}{r} \frac{\partial}{\partial r}+\frac{1}{r^{2}} \frac{\partial^{2}}{\partial \theta^{2}}-\frac{1}{\nu} \frac{\partial}{\partial t}\right) \chi_{2}=0, \\
\text { and } \quad d p=\rho \frac{\partial}{\partial t}\left(\frac{\partial \chi_{1}}{\partial r} r d \theta+\frac{1}{r} \frac{\partial \chi_{1}}{\partial \theta} d r\right),
\end{gathered}
$$

where $v_{r}$ is the radial velocity, $v_{\text {theta }}$ is the tangential velocity, and $\chi=\chi_{1}+\chi_{2}$ is the stream function. Given the cylinder's motion, $V_{c}$ (equation A.5), Stokes solution for $\chi$ is

$$
\chi_{1}=e^{i \omega t} \sin \theta F_{1}(r), \quad \chi_{2}=e^{i \omega t} \sin \theta F_{2}(r),
$$

where the governing equations with boundary conditions become

$$
\begin{gathered}
F_{1}^{\prime \prime}(r)+\frac{1}{r} F_{1}^{\prime}(r)-\frac{1}{r^{2}} F_{1}(r)=0, \\
F_{2}^{\prime \prime}(r)+\frac{1}{r} F_{2}^{\prime}(r)-\frac{1}{r^{2}} F_{2}(r)-m^{2} F_{2}(r)=0, \\
F_{1}(a)+F_{2}(a)=a U_{o} \text { and } F_{1}^{\prime}(a)+F_{2}^{\prime}(a)=U_{o},
\end{gathered}
$$

where $m^{2}=i \omega / \nu$. Stokes was finally able to solve these differential equations when he let $F_{2}(r)=F_{3}^{\prime}(r)$, giving

$$
F_{3}^{\prime \prime}(r)+\frac{1}{r} F_{3}^{\prime}(r)-m^{2} F_{3}(r)=0
$$

and applied the solution he had found for that from pursuing another problem. Skipping a great many of his steps and continuing to develop the solution that will enable calculation of the pressure and velocity fields,

$$
F_{1}(r)=\frac{\Lambda}{r}, \quad \Lambda=a^{2} U_{o}\left(1-\frac{2 F_{3}^{\prime}(a)}{a m^{2} F_{3}(a)}\right)
$$

For a solution for $F_{3}(r)$ which converges for all values of $r$,

$$
F_{3}(r)=(C+D \log r)\left(1+\frac{m^{2} r^{2}}{2^{2}}+\frac{m^{4} r^{4}}{2^{2} \cdot 4^{2}}+\ldots\right)-D\left(\frac{1}{1} \cdot \frac{m^{2} r^{2}}{2^{2}}+\frac{1}{2} \cdot \frac{m^{4} r^{4}}{2^{2} \cdot 4^{2}}+\frac{1}{3} \cdot \frac{m^{6} r^{6}}{2^{2} \cdot 4^{2} \cdot 6^{2}}+\ldots\right)
$$


where $C$ and $D$ are related according to

$$
C=\left(\log \frac{m}{8}-\frac{1}{\sqrt{\pi}} \Gamma^{\prime}\left(\frac{1}{2}\right)\right) D
$$

Each of $C$ or $D$ and can be determined from the governing equations with boundary conditions applied (from equations A.11),

$$
\frac{\Lambda}{a}+F_{3}^{\prime}(a)=a U_{o}
$$

Letting $F_{3}^{\prime}(a)=C \sigma$, then

$$
C=\frac{1}{\sigma}\left(a U_{o}-\Lambda / a\right)
$$

and with $\zeta=D / C$ (determined by equation A.15), then

$$
\begin{gathered}
\sigma=\frac{\zeta}{a}\left(1+\frac{m^{2} a^{2}}{2^{2}}+\frac{m^{4} a^{4}}{2^{2} \cdot 4^{2}}+\ldots\right)+(1+\zeta \log a)\left(2 \frac{m^{2} a^{1}}{2^{2}}+4 \frac{m^{4} a^{3}}{2^{2} \cdot 4^{2}}+\ldots\right) \\
-\zeta\left(\frac{2}{1} \cdot \frac{m^{2} a}{2^{2}}+\frac{4}{2} \cdot \frac{m^{4} a^{3}}{2^{2} \cdot 4^{2}}+\frac{6}{3} \cdot \frac{m^{6} a^{5}}{2^{2} \cdot 4^{2} \cdot 6^{2}}+\ldots\right) .
\end{gathered}
$$

When the modulus $m r$ is large, $F_{3}(r)$ can be integrated by exponentials combined with a descending series, which with the condition that the velocity must vanish at infinity gives

$$
F_{3}(r)=\Upsilon \frac{e^{-m r}}{\sqrt{r}}\left(1-\frac{1^{2}}{2 \cdot(4 m r)}+\frac{1^{2} \cdot 3^{2}}{2 \cdot 4 \cdot(4 m r)^{2}}-\frac{1^{2} \cdot 3^{2} \cdot 5^{2}}{2 \cdot 4 \cdot 6 \cdot(4 m r)^{3}}+\ldots\right)
$$

Letting $F_{3}^{\prime}(a)=\Upsilon \varphi$, and duplicating the previous steps,

$$
\Upsilon=\frac{1}{\varphi}\left(a U_{o}-\Lambda / a\right)
$$

with

$$
\varphi=\frac{e^{-m a}}{\sqrt{a}}\left(\frac{1}{a} \cdot \frac{1^{2}}{2 \cdot(4 m a)}-\frac{2}{a} \cdot \frac{1^{2} \cdot 3^{2}}{2 \cdot 4 \cdot(4 m a)^{2}}+\frac{3}{a} \cdot \frac{1^{2} \cdot 3^{2} \cdot 5^{2}}{2 \cdot 4 \cdot 6 \cdot(4 m a)^{3}}+\ldots\right)
$$


$+\left(-m \frac{e^{-m a}}{\sqrt{a}}-\frac{1}{2 a} \frac{e^{-m a}}{\sqrt{a}}\right)\left(1-\frac{1^{2}}{2 \cdot(4 m a)}+\frac{1^{2} \cdot 3^{2}}{2 \cdot 4 \cdot(4 m a)^{2}}-\frac{1^{2} \cdot 3^{2} \cdot 5^{2}}{2 \cdot 4 \cdot 6 \cdot(4 m a)^{3}}+\ldots\right)$.

Having now determined $\Lambda(a, \omega), \chi_{1}$ is solved, and the total pressure differential can be integrated to give

$$
p(r, \theta, t)=i \rho \omega e^{i \omega t} \Lambda \frac{\cos \theta}{r} .
$$

Having also determined $C$ and $\Upsilon, \chi_{2}$ is effectively solved for when the modulus $m r$ is small and large respectively. The radial and tangential velocity fields can no be determined.

$$
v_{r}(r, \theta, t)=\frac{1}{r} \frac{\partial \chi}{\partial \theta}=\cos \theta\left(\frac{\Lambda}{r^{2}}+\frac{F_{3}^{\prime}(r)}{r}\right) e^{i \omega t}
$$

which can be expressed as the potential flow solution multiplied by a correction term (in brackets)

$$
v_{r}(r, \theta, t)=U_{o}\left(\frac{a}{r}\right)^{2} \cos \theta\left[1-\frac{2 F_{3}^{\prime}(a)}{m^{2} a F_{3}(a)}+\frac{r F_{3}^{\prime}(r)}{a^{2} U_{o}}\right] e^{i \omega t}
$$

Likewise,

$$
v_{\theta}=-\frac{\partial \chi}{\partial r}=\sin \theta\left(\frac{\Lambda}{r^{2}}-F_{3}^{\prime \prime}(r)\right) e^{i \omega t}
$$

which can also be expressed as the potential flow solution multiplied by a correction term (in brackets)

$$
v_{\theta}(r, \theta, t)=U_{o}\left(\frac{a}{r}\right)^{2} \sin \theta\left[1-\frac{2 F_{3}^{\prime}(a)}{m^{2} a F_{3}(a)}-\frac{r^{2} F_{3}^{\prime \prime}(r)}{a^{2} U_{o}}\right] e^{i \omega t}
$$

Finally, Stokes gives the following formula for the drag:

$$
F=-i \omega \rho \pi a^{2} U_{o}\left[1-\frac{4 F_{3}^{\prime}(a)}{m^{2} a F_{3}(a)}\right] e^{i \omega t}
$$




\section{Bibliography}

[Abbott and von Doenhoff, 1959] Abbott, I. H. and von Doenhoff, A. E. (1959). Theory of Wing Sections: Including a summary of airfoil data. Dover, New York.

[Anderson et al., 2001] Anderson, E. J., McGillis, W., and Grosenbaugh, M. (2001). The boundary layer of swimming fish. J. Exp. Bio, 204(1):81-102.

[Badr, 994a] Badr, H. M. (1994a). Oscillating inviscid flow over elliptic cylinders with flat plates and circular cylinders as special cases. Ocean Engineering, 21(1):105113.

[Badr, 994b] Badr, H. M. (1994b). Oscillating viscous flow over an inclined elliptic cylinder. Ocean Engineering, 21(4):401-426.

[Badr and Kocabiyik, 1997] Badr, H. M. and Kocabiyik, S. (1997). Symmetrically oscillating viscous flow over an elliptic cylinder. J. Fluids and Structures, 11:745766.

[Barbier and Humphrey, 2006] Barbier, C. and Humphrey, J. A. C. (2006). Numerical calculation of the flow in the fish lateral line canal: applications to preditors tracking prey. In Proceedings of IMECE2006, International Mechanical Engineering Congress and Exposition, pages 283-292, Chicago.

[Barth and Jespersen, 1989] Barth, T. J. and Jespersen, D. (1989). The design and application of upwind schemes on unstructured meshes. AIAA 27th Aerospace Sciences Meeting, pages Technical Report AIAA-89-0366. 
[Bassett et al., 2006] Bassett, D. K., Carton, A. G., and Montgomery, J. C. (2006). Flowing water decreases hydrodynamic signal detection in a fish with an epidermal lateral-line system. Marine And Freshwater Research, 57(6):611-617.

[Bleckmann, 1986] Bleckmann, H. (1986). Role of the lateral line in fish behavior. In The Behavior of Teleost Fishes, pages 177-202.

[Bleckmann et al., 2003] Bleckmann, H., Mogdans, J., and Dehnhardt, G. (2003). Processing of dipole and more complex hydrodynamic stimuli under still-and running-water conditions. Collin SP, Marshall NJ. New York: Springer, pages $108-121$.

[Bodznick et al., 2003] Bodznick, D., Montgomery, J. C., and Tricas, T. C. (2003). Electroreception: extracting behaviorally important signals from noise. In Collin, S. P. and Marshall, N. J., editors, Sensory Processing in Aquatic Environments, pages 389-403. Springer, New York.

[Braun and Coombs, 2000] Braun, C. B. and Coombs, S. (2000). The overlapping roles of the inner ear and lateral line: the active space of dipole source detection. Philosophical Transactions: Biological Sciences, 355(1401):1115-1119.

[Brennan, 1982] Brennan, C. E. (1982). A review of added mass and fluid inertial forces. page Government publication CR 82.010.

[Cahn, 1967] Cahn, P. (1967). Preface. In Lateral Line Detectors, pages v - vi. Indiana University Press.

[Campenhausen von et al., 1981] Campenhausen von, C., Riess, I., and Weissert, R. (1981). Detection of stationary objects by the blind cave fishanoptichthys jordani (characidae). Journal of Comparative Physiology A: Sensory, Neural, and Behavioral Physiology, 143(3):369-374.

[Carling et al., 1998] Carling, J., Williams, T., and Bowtell, G. (1998). Self-propelled anguilliform swimming: simultaneous solution of the two-dimensional navier- 
stokes equations and newton's laws of motion. Journal of Experimental Biology, 201(23):3143-3166.

[Chagnaud et al., 2008] Chagnaud, B. P., Bleckmann, H., and Hofmann, M. H. (2008). Lateral line nerve fibers do not code bulk water flow direction in turbulent flow. Zoologische Jahrbucher. Abteilung fur Anatomie und Ontogenie der Tiere, 111:204-217.

[Chagnaud et al., 2006] Chagnaud, B. P., Hofmann, M. H., and Mogdans, J. (2006). Responses to dipole stimuli of anterior lateral line nerve fibres in goldfish, carassius auratus, under still and running water conditions. Journal of Comparative Physiology A: Neuroethology, Sensory, Neural, and Behavioral Physiology, 193(2):249-263.

[Conley and Coombs, 1998] Conley, R. A. and Coombs, S. (1998). Dipole source localization by mottled sculpin. iii. orientation after site-specific, unilateral denervation of the lateral line system. Journal of Comparative Physiology.A, Sensory, neural, and behavioral physiology, 183(3):335-344. LR: 20061115; PUBM: Print; JID: 8413199; ppublish Journal.

[Coombs et al., 2007] Coombs, S., Anderson, E., Braun, C. B., and Grosenbaugh, M. (2007). The hydrodynamic footprint of a benthic, sedentary fish in unidirectional flow. The Journal of the Acoustical Society of America, 122:1227.

[Coombs and Braun, 2003] Coombs, S. and Braun, C. B. (2003). Information processing by the lateral line system. Sensory Processing in Aquatic Environments, pages $174-182$.

[Coombs et al., 2001] Coombs, S., Braun, C. B., and Donovan, B. (2001). The orienting response of lake michigan mottled sculpin is mediated by canal neuromasts. Journal Of Experimental Biology, 204(2):337-348.

[Coombs and Conley, 997a] Coombs, S. and Conley, R. A. (1997a). Dipole source localization by mottled sculpin. i. approach strategies. Journal of Comparative Physiology.A, Sensory, Neural, and Behavioral Physiology, 180(4):387-399. 
[Coombs and Conley, 997b] Coombs, S. and Conley, R. A. (1997b). Dipole source localization by the mottled sculpin. ii. the role of lateral line excitation patterns. Journal of Comparative Physiology.A, Sensory, Neural, and Behavioral Physiology, 180(4):401-415.

[Coombs et al., 2000] Coombs, S., Finneran, J. J., and Conley, R. A. (2000). Hydrodynamic image formation by the peripheral lateral line system of the lake michigan mottled sculpin, cottus bairdi. Philosophical transactions of the Royal Society of London.Series B, Biological sciences, 355(1401):1111-1114.

[Coombs et al., 1996] Coombs, S., Hastings, M., and Finneran, J. (1996). Modeling and measuring lateral line excitation patterns to changing dipole source locations. Journal of Comparative Physiology.A, Sensory, Neural, and Behavioral Physiology, 178(3):359-371.

[Coombs and Janssen, 1990] Coombs, S. and Janssen, J. (1990). Behavioral and neurophysiological assessement of lateral line sensitivity in the mottled sculpin, cottus bairdi. J. Comp Physiol A, 167:557-567.

[Coombs and Montgomery, 1999] Coombs, S. and Montgomery, J. C. (1999). The enigmatic lateral line system. Comparative Hearing: Fishes and Amphibians (ed. by AN Popper and RR Fay) Springer Handbook of Auditory Research, 11:319362.

[Curcic-Blake and van Netten, 2005] Curcic-Blake, B. and van Netten, S. M. (2005). Rapid responses of the cupula in the lateral line of ruffe (gymnocephalus cernuus). Journal of Comparative Physiology A: Sensory, Neural, and Behavioral Physiology, 191(4):393-401.

[Curcic-Blake and van Netten, 2006] Curcic-Blake, B. and van Netten, S. M. (2006). Source location encoding in the fish lateral line canal. The Journal of Experimental Biology, 209(Pt 8):1548-1559. 
[Demirdzic and Peric, 1990] Demirdzic, I. and Peric, M. (1990). Finite volume method for prediction of fluid flow in arbitrarily shaped domains with moving boundaries. Int. J. Num. Methods in Fluids, 10:771790.

[Denton and Gray, 1983] Denton, E. J. and Gray, J. (1983). Mechanical factors in the excitation of clupeid lateral lines. Proceedings of the Royal Society of London. Series B, Biological Sciences, 218(1210):1-26.

[Denton and Gray, 1982] Denton, E. J. and Gray, J. A. B. (1982). The rigidity of fish and patterns of lateral line stimulation. Nature, 297(5868):679-681.

[Denton and Gray, 1993] Denton, E. J. and Gray, J. A. B. (1993). Stimulation of the acoustico-lateralis system of clupeid fish by external sources and their own movements. Philosophical Transactions Of The Royal Society Of London Series B-Biological Sciences, 341(1296):113-127.

[Dijkgraaf, 1967] Dijkgraaf, S. (1967). Biological significance of the lateral line organs. Lateral Line Detectors (Cahn P, ed), page 8395.

[Dinklo, 2005] Dinklo, T. (2005). Mechano- and electrophysiological studies on cochlear hair cells and lateral line cupulae. PhD thesis, Rijksuniversiteit Groningen.

[Eatock, 2000] Eatock, R. (2000). Adaptation in hair cells. Annu. Rev. Neurosci., 23:285-314.

[Eatock et al., 1987] Eatock, R., Corey, D. P., and Hudspeth, A. J. (1987). Adaptation of mechanoeletrical transduction in hair cells of the bullfrog's sacculus. $J$. Neuroscience, 7(9):2821-2836.

[Engelmann et al., 2002] Engelmann, J., Hanke, W., and Bleckmann, H. (2002). Lateral line reception in still-and running water. Journal of Comparative Physiology A: Sensory, Neural, and Behavioral Physiology, 188(7):513-526.

[Engelmann et al., 2000] Engelmann, J., Hanke, W., Mogdans, J., and Bleckmann, H. (2000). Hydrodynamic stimuli and the fish lateral line. Nature, 408(6808):51-2. 
[Engelmann et al., 2003] Engelmann, J., Krother, S., Bleckmann, H., and Mogdans, J. (2003). Effects of running water on lateral line responses to moving objects. Brain, Behav Evol, 61(4):195-212.

[Englemann et al., 2003] Englemann, H., Mogdans, J., and Dehnhardt, G. (2003). Processing of dipole and more complex hydrodynamic stimuli under still- and running- water conditions. In Sensory Processing in Aquatic Environments, pages $108-121$.

[Fay, 1990] Fay, R. R. (1990). Suppression and excitation in auditory nerve fibers of the goldfish, carassius auratus. Hearing Research, 48:93-110.

[Fay, 1997] Fay, R. R. (1997). Frequency selectivity of saccular afferents of teh goldfish revealed by revcor analysis. In Lewis, E., Long, G. R., Lyon, R. F., Narins, P. M., Steele, C. R., and Hecht-Poinar, E., editors, Diversity in auditory mechanics, pages 69-75. World Scientific Publishers, Singapore.

[Ferzieger and Peric, 1996] Ferzieger, J. L. and Peric, M. (1996). Computational Methods for Fluid Dynamics. Springer-Verlag.

[Flock, 1967] Flock, A. (1967). Ultrastructure and function in the lateral line organs. Lateral Line Detectors, page 163197.

[Flock and Duvall, 1965] Flock, A. and Duvall, A. J. (1965). The ultrastructure of the kinocilium of the sensory cells in the inner ear and lateral line organs. The Journal of Cell Biology, 25(1):1-8.

[Flock and Wersall, 1962] Flock, A. and Wersall, J. (1962). A study of the orientation of the sensory hairs of the receptor cells in the lateral line organ of fish, with special reference to the function of the receptors. The Journal of Cell Biology, 15(1):19-27.

[Franosch et al., 2005] Franosch, J. M. P., Sichert, A. B., Suttner, M. D., and van Hemmen, J. L. (2005). Estimating position and velocity of a submerged moving object by the clawed frog xenopus and by fisha cybernetic approach. Biological Cybernetics, 93(4):231-238. 
[Furukawa, 1986] Furukawa, T. (1986). Sound reception and synaptic transmission in goldfish hair cells. Japanese Journal of Physiology, 36:1059-1077.

[Gilmanov and Sotiropoulos, 2005] Gilmanov, A. and Sotiropoulos, F. (2005). A hybrid cartesian/immersed boundary method for simulating flows with 3d, geometrically complex, moving bodies. Journal of Computational Physics, 207(2):457-492.

[Gorner, 1967] Gorner, P. (1967). Independence of afferent activity from efferent activity in the lateral line organ of xenopus laevis daudin. In Lateral Line Detectors, pages $199-214$.

[Goulet et al., 2008] Goulet, J., Engelmann, J., Chagnaud, B. P., Franosch, J. M. P., Suttner, M. D., and Hemmen, J. L. (2008). Object localization through the lateral line system of fish: theory and experiment. J. Comp Physiol A, 194:1-17.

[Grant and Madsen, 1986] Grant, W. D. and Madsen, O. S. (1986). The continentalshelf bottom boundary layer. Ann. Rev. Fluid Mech, 18:265-305.

[Gus'kova et al., 1998] Gus'kova, N. Y., Makhortykh, G. V., and Shcheglova, M. G. (1998). Inertia and drag of elliptic cylinders oscillating in a fluid. Fluid Dynamics, 23(1):91-95.

[Hanke et al., 2000] Hanke, W., Brucker, C., and Barth, F. G. (2000). The ageing of the low-frequency water disturbances caused by swimming goldfish and its possible relevance to prey detection. Journal of Experimental Biology, 203(7):1193-1200.

[Harris and Flock, 1967] Harris, G. G. and Flock, A. (1967). Spontaneous and evoked activity from the xenopus laevis lateral line. Lateral Line Detectors, page 135162.

[Hassan, 1985] Hassan, E. S. (1985). Mathematical analysis of the stimulus for the lateral line organ. Biological Cybernetics, 52(1):23-36.

[Hassan, 1992a] Hassan, E. S. (1992a). Mathematical description of the stimuli to the lateral ine system of fish derived from a three-dimensional flow field analysis. 
ii: The case of gliding alongside or above a plane surface. Biological Cybernetics, 66(5):453-461. Journal.

[Hassan, 1992b] Hassan, E. S. (1992b). Mathematical description of the stimuli to the lateral line system of fish derived from a three-dimensional flow field analysis i: the cases of moving in open water and of gliding towards a plane surface. Biological Cybernetics, 66(5):443-452. Journal.

[Hassan, 1993] Hassan, E. S. (1993). Mathematical description of the stimuli to the lateral line system of fish, derived from a three-dimensional flow field analysis. iii. the case of an oscillating sphere near the fish. Biological Cybernetics, 69(5):525-538. Journal.

[Howard and Hudspeth, 1987] Howard, J. and Hudspeth, A. J. (1987). Mechanical relaxation of the hair bundle mediates adaptation in mechanoelectrical transduction of the bullfrog's saccular hair cell. In Proc. Natl. Acad. Sci.: Neurobiology, volume 84, pages 3064-3068.

[Humphrey et al., 2001] Humphrey, J. A. C., Barth, F. G., and Voss, K. (2001). The motion-sensing hairs of arthropods: using physics to understand sensory ecology and adaptive evolution. Ecology of sensing. Springer, Berlin, page 105125.

[Humphrey et al., 1993] Humphrey, J. A. C., Devarakonda, R., Iglesias, I., and Barth, F. G. (1993). Dynamics of arthropod filiform hairs. i. mathematical modelling of the hair and air motions. Philosophical Transactions: Biological Sciences, 340(1294):423-444.

[Issa, 1986] Issa, R. I. (1986). Solution of implicitly discretized fluid flow equations by operator splitting. J. Comput. Phys., 62:40-65.

[Jacobs et al., 1933] Jacobs, E. N., Ward, K. E., and Pinkerton, R. M. (1933). The characteristics of 78 related airfoil sections from tests in the variable density wind tunnel. NASA:Report Number NACA-TR-460. 
[Janssen, 2004] Janssen, J. (2004). Lateral line sensory ecology. The Senses of Fish: Adaptations for the Reception of Natural Stimuli. Narosa Publishing House, New Delhi.

[Janssen et al., 1987] Janssen, J., Coombs, S., Hoekstra, C., and Platt, C. (1987). Anatomy and differential growth of the lateral line system of the mottled sculpin, cotttus bairdi, (scorpaeniformes: Cottidae). Brain Behavior And Evolution, $30: 210-229$.

[Jiang et al., 2002] Jiang, H. S., Meneveau, C., and Osborn, T. R. (2002). The flow field around a freely swimming copepod in steady motion. part ii: Numerical simulation. Journal Of Plankton Research, 24(3):191-213.

[Kalmijn, 1988] Kalmijn, A. J. (1988). Hydrodynamic and acoustic field detection. Sensory Biology of Aquatic Animals, pages 83-130. Journal.

[Kanter and Coombs, 2003] Kanter, M. J. and Coombs, S. (2003). Rheotaxis and prey detection in uniform currents by lake michigan mottled sculpin (cottus bairdi). The Journal of Experimental Biology, 206(Pt 1):59-70.

[Kroese et al., 1978] Kroese, A. B. A., van der Zalm, J. M., and van den Bercken, J. V. (1978). Frequency response of the lateral-line organ of xenopus laevis. Pflugers Archiv: European Journal of Physiology, 375:167-175.

[Kroese and van Netten, 1989] Kroese, A. B. A. and van Netten, S. M. (1989). Sensory transduction in lateral line hair cells. In Coombs, S., Gorner, P., and Munz, H., editors, The Mechanosensory Lateral Line: Neurobiology and Evolution., pages 265-284. Springer, New York.

[Kuiper, 1967] Kuiper, J. W. (1967). Frequency characteristics and functional significance of the lateral line organ. Lateral line detectors. Indiana University Press, Bloomington London, pages 105-121.

[Lamb, 1945] Lamb, H. (1945). Hydrodynamics. Dover Publications. 
[Leonard and Mokhtari, 1990] Leonard, B. P. and Mokhtari, S. (1990). Ultra-sharp nonoscillatory convection schemes for high-speed steady multidimensional flow. NASA Lewis Research Center:NASA TM 1-2568 (ICOMP-90-12).

[Liao, 2004] Liao, J. C. (2004). Neuromuscular control of trout swimming in a vortex street: implications for energy economy during the karman gait. Journal of Experimental Biology, 207(20):3495-3506.

[Liao, 2006] Liao, J. C. (2006). The role of the lateral line and vision on body kinematics and hydrodynamic preference of rainbow trout in turbulent flow. Journal of Experimental Biology, 209(20):4077.

[Liao et al., 2003a] Liao, J. C., Beal, D. N., Lauder, G. V., and Triantafyllou, M. S. (2003a). Fish exploiting vortices decrease muscle activity. Science, 302(5650):15661569.

[Liao et al., 2003b] Liao, J. C., Beal, D. N., Lauder, G. V., and Triantafyllou, M. S. (2003b). The karman gait: novel body kinematics of rainbow trout swimming in a vortex street. Journal of Experimental Biology, 206(6):1059-1073.

[Lighthill, 1978] Lighthill, J. (1978). Acoustic streaming. Journal Of Sound And Vibration, 61(3):391-418.

[Liu et al., 1997] Liu, H., Wassersug, R., and Kawachi, K. (1997). The threedimensional hydrodynamics of tadpole locomotion. Journal of Experimental Biology, 200(22):2807-2819.

[McHenry and van Netten, 2007] McHenry, M. J. and van Netten, S. M. (2007). The flexural stiffness of superficial neuromasts in the zebrafish (danio rerio) lateral line. J. Exp. Biol, 210:4244-4253.

[Mller et al., 1996] Mller, H. M., Fleck, A., and Bleckmann, H. (1996). The responses of central octavolateralis cells to moving sources. Journal of Comparative Physiology A: Sensory, Neural, and Behavioral Physiology, 179(4):455-471. 
[Mogdans and Bleckmann, 1998] Mogdans, J. and Bleckmann, H. (1998). Responses of the goldfish trunk lateral line to moving objects. Journal Of Comparative Physiology A-Sensory Neural And Behavioral Physiology, 182(5):659-676.

[Montgomery et al., 1997] Montgomery, J. C., Baker, C. F., and Carton, A. G. (1997). The lateral line can mediate rheotaxis in fish. Nature(London), 389(6654):960-963.

[Montgomery and Coombs, 1998] Montgomery, J. C. and Coombs, S. (1998). Peripheral enoding of moving sources by the lateral line system of a sit-and-wait predator. Journal of Experimental Biology, 201:91-102.

[Montgomery et al., 1988] Montgomery, J. C., Macdonald, J. A., and Housley, G. D. (1988). Lateral line function in an antarctic fish related to the signals produced by planktonic prey. Journal of Comparative Physiology A: Sensory, Neural, and Behavioral Physiology, 163(6):827-833.

[Nair and Sengupta, 1997] Nair, M. T. and Sengupta, T. K. (1997). Unsteady flow past elliptic cylinders. J. Fluids and Structures, 11:555-595.

[Nam et al., 2007] Nam, J.-H., Cotton, J. R., and Grant, W. (2007). A virtual hair cell, i: addition of gating spring theory into a 3-d bundle mechanical model. Biophysical Journal, 92:1918-1928.

[Nelson and MacIver, 1999] Nelson, M. E. and MacIver, M. A. (1999). Prey capture in the weakly electric fish apteronotus leptorhynchus: sensory acquisition strategies and electrosensory consequences. J. Exp. Biol, 202:11951203.

[Palmer et al., 2005] Palmer, L. M., Deffenbaugh, M., and Mensinger, A. F. (2005). Sensitivity of the anterior lateral line to natural stimuli in the oyster toadfish, opsanus tau (linnaeus). Journal Of Experimental Biology, 208(18):3441-3450.

[Pan and Chew, 2002] Pan, L. S. and Chew, Y. T. (2002). A general formula for calculating forces on a 2-d arbitrary body in incompressible flow. J. Fluids and Structures, 16(1):71-82. 
[Partridge and Pitcher, 1980] Partridge, B. L. and Pitcher, T. J. (1980). The sensory basis of fish schools: Relative roles of lateral line and vision. Journal of Comparative Physiology A: Sensory, Neural, and Behavioral Physiology, 135(4):315-325.

[Patankar, 1980] Patankar, S. V. (1980). Numerical Heat Transfer and Fluid Flow. Hemisphere.

[Pohlmann et al., 2004] Pohlmann, K., Atema, J., and Breithaupt, T. (2004). The importance of the lateral line in nocturnal predation of piscivorous catfish. Journal Of Experimental Biology, 207(17):2971-2978.

[Popper and Fay, 1999] Popper, A. N. and Fay, R. R. (1999). The auditory periphery in fishes. In Fay, R. R. and Popper, A. N., editors, Comparative Hearing: Fish and Amphibians, pages xi-xiii. Springer-Verlog, New York.

[Richards, 1934] Richards, G. T. (1934). On the motion of an elliptic cylinder through a viscous fluid. Philosophical Transactions Of The Royal Society Of London Series A, Containing Papers of a Mathematical or Physical Character, 233:279-301.

[Riley, 1965] Riley, N. (1965). Oscillating viscous flows. Mathematika, 12:161-175.

[Riley, 1966] Riley, N. (1966). On a sphere oscillating in a viscous fluid. The Quarterly Journal of Mechanics and Applied Mathematics, 19(4):461.

[Roberts and Rutherford, 2008] Roberts, W. M. and Rutherford, M. A. (2008). Linear and nonlinear processing in hair cells. J. Exp. Biol, 211:1775-1780.

[Schlichting, 1979] Schlichting, H. (1979). Boundary Layer Theory. McGraw-Hill, New York.

[Stokes, 1851] Stokes, G. G. (1851). On the effect of internal friction of fluids on the motion of pendulums. Transactions of the Cambridge Philosophical Society, IX.

[Tatsuno and Bearman, 1990] Tatsuno, M. and Bearman, P. W. (1990). A visual study of the flow around an oscillating circular-cylinder at low keulegan-carpenter numbers and low stokes numbers. Journal Of Fluid Mechanics, 211:157-182. 
[Teyke, 1988] Teyke, T. (1988). Flow field, swimming velocity and boundary layer: parameters which affect the stimulus for the lateral line organ in blind fish. Journal of Comparative Physiology A: Sensory, Neural, and Behavioral Physiology, $163(1): 53-61$.

[Tinevez et al., 2007] Tinevez, J.-Y., Julicher, F., and Martin, P. (2007). Unifying the various incarnations of active hair-bundle motility by the vertebrate hair cell. Biophysical Journal, 93:4053-4067.

[van Netten, 1991] van Netten, S. M. (1991). Hydrodynamics of the excitation of the cupula in the fish canal lateral line. J. Accoustical Society of America, 89(1):310319.

[van Netten, 2006] van Netten, S. M. (2006). Hydrodynamic detection by cupulae in a lateral line canal: functional relations between physics and physiology. Biological Cybernetics, 94(1):67-85.

[van Netten and Kroese, 1987] van Netten, S. M. and Kroese, A. B. A. (1987). Laser interferometric measurements on the dynamic behaviour of the cupula in the fish lateral line. Hearing Research, 29:55-61.

[Visser, 2001] Visser, A. W. (2001). Hydromechanical signals in the plankton. Marine Ecology-Progress Series, 222:1-24.

[Voigt et al., 2000] Voigt, R., Carton, A. G., and Montgomery, J. C. (2000). Responses of anterior lateral line afferent neurones to water flow. Journal Of Experimental Biology, 203(16):2495-2502.

[Walker and Westneat, 2000] Walker, J. A. and Westneat, M. W. (2000). Mechanical performance of aquatic rowing and flying. Proceedings: Biological Sciences, 267(1455):1875-1881.

[Weeg and Bass, 2002] Weeg, M. S. and Bass, A. H. (2002). Frequency response properties of lateral line superficial neuromasts in a vocal fish, with evidence for acoustic sensitivity. Journal of Neurophysiology, 88(3):1252-1262. 
[Welch, 1967] Welch, P. D. (1967). The use of fast fourier transforms for the estimation of power spectra: A method based on time averaging over short modified periodograms. IEEE Transactions on Audio and Electroacoustics, 15:70-73.

[Windsor, 2008] Windsor, S. (2008). Hydrodynamic Imaging of the Blind Mexican Cave Fish. PhD thesis, The University of Auckland.

[Wolfgang et al., 1999] Wolfgang, M. J., Anderson, J., Grosenbaugh, M., Yue, D. K. P., and Triantafyllou, M. S. (1999). Near-body flow dynamics in swimming fish. Journal of Experimental Biology, 202(17):2303-2327.

[Yang et al., 2006] Yang, Y., Chen, J., Engel, J., Pandya, S., Chen, N., Tucker, C., Coombs, S., Jones, D. L., and Liu, C. (2006). Distant touch hydrodynamic imaging with an artificial lateral line. Proceedings of the National Academy of Sciences of the United States of America, 103(50):18891-18895.

[Zhu et al., 2002] Zhu, Q., Wolfgang, M. J., Yue, D. K. P., and Triantafyllou, M. S. (2002). Three-dimensional flow structures and vorticity control in fish-like swimming. Journal Of Fluid Mechanics, 468:1-28. 